\title{
MOVEMENTS AND OCEANOGRAPHIC ASSOCIATIONS OF LARGE PELAGIC Fishes IN The North AtlantiC OCEAN
}

\author{
by \\ Camrin Donald Braun \\ M.S., King Abdullah University of Science and Technology (2013) \\ B.S., The College of Idaho (2011) \\ Submitted in partial fulfillment of the requirements for the degree of \\ Doctor of Philosophy \\ at the \\ MASSACHUSETTS INSTITUTE OF TECHNOLOGY \\ and the \\ WOODS HOLE OCEANOGRAPHIC INSTITUTION
}

September 2018

(C)2018 Camrin Donald Braun. All rights reserved.

The author hereby grants to MIT and WHOI permission to reproduce and to distribute publicly paper and electronic copies of this thesis document in whole or in part in any medium now known or hereafter created.

Author

Joint Program in Applied Ocean Science \& Engineering Massachusetts Institute of Technology \& Woods Hole Oceanographic Institution

August 10, 2018

Certified by

Simon R. Thorrold

Senior Scientist

Woods Hole Oceanographic Institution

Thesis Supervisor

Accepted by

Ann Tarrant

Chair, Joint Committee for Biological Oceanography Massachusetts Institute of Technology

Woods Hole Oceanographic Institution 
THIS PAGE INTENTIONALLY LEFT BLANK 


\title{
MOVEMENTS AND OCEANOGRAPHIC ASSOCIATIONS OF LARGE PELAGIC Fishes IN THE NoRTh Atlantic OCEAN \\ by \\ Camrin Donald Braun
}

\begin{abstract}
Submitted to the MIT-WHOI Joint Program in Oceanography and Applied Ocean Science and Engineering on August 10, 2018, in partial fulfillment of the requirements for the degree of Doctor of Philosophy in Biological Oceanography
\end{abstract}

\begin{abstract}
Highly migratory marine fishes support valuable commercial fisheries worldwide. Yet, many target species have proven difficult to study due to long-distance migrations and regular deep diving. Despite the dominance of oceanographic features, such as fronts and eddies, in the open ocean, the biophysical interactions occurring at the oceanic (sub)mesoscale $(<100 \mathrm{~km})$ remain poorly understood. This leads to a paucity of knowledge on oceanographic associations of pelagic fishes and hinders management efforts. With ever-improving oceanographic datasets and modeling outputs, we can leverage these tools both to derive better estimates of animal movements and to quantify fish-environment interactions. In this thesis, I developed analytical tools to characterize the biophysical interactions influencing animal behavior and species' ecology in the open ocean. A novel, observation-based likelihood framework was combined with a Bayesian state-space model to improve geolocation estimates for archival-tagged fishes using oceanographic profile data. Using this approach, I constructed track estimates for a large basking shark tag dataset using a high-resolution oceanographic model and discovered a wide range of movement strategies. I also applied this modeling approach to track archival-tagged swordfish, which revealed affinity for thermal front and eddy habitats throughout the North Atlantic that was further corroborated by synthesizing these results with a fisheries-dependent conventional tag dataset. An additive modeling approach applied to longline catch-per-unit effort data further highlighted the biophysical interactions that characterize variability in swordfish catch. In the final chapter, I designed a synergistic analysis of high-resolution, 3D shark movements and satellite observations to quantify the influence of mesoscale oceanography on blue shark movements and behavior. This work demonstrated the importance of eddies in structuring the pelagic ocean by influencing the movements of an apex predator and governing the connectivity between deep scattering layer communities and deep-diving, epipelagic predators. Together, these studies demonstrate the breadth and depth of information that can be garnered through the integration of traditional animal tagging and oceanographic research with cutting-edge analytical approaches and high-resolution oceanographic model and remote sensing datasets, the product of which provides a transformative view of the biophysical interactions occurring in and governing the structure of the pelagic ocean.
\end{abstract}

Thesis Supervisor: Simon R. Thorrold

Title: Senior Scientist

Woods Hole Oceanographic Institution 
THIS PAGE INTENTIONALLY LEFT BLANK 
For Mako, you kept me grounded. 
THIS PAGE INTENTIONALLY LEFT BLANK 


\section{ACKNOWLEDGMENTS}

During my time in the MIT-WHOI Joint Program, I have been supported by the NASA Earth and Space Science Fellowship, the MIT John S. Hennessy Fellowship, the MIT Martin Family Society of Fellows for Sustainability Fellowship, the WHOI Ocean Venture, Grassle, and James Stratton Fellowships and the WHOI Academic Programs Office. This research and its dissemination was supported by funds from National Geographic, Amazon Web Services, the Explorers Club, Rolex, Sigma Xi, the MIT Center for International Studies, WHOI Access to the Sea and Coastal Ocean Institute Funds, MIT Graduate Student Council, MIT Student Assistance Fund, WHOI Biology Department, American Fisheries Society, WHOI Academic Programs Office and many individual donors of time and resources. For all of your support, large and small, I am eternally grateful.

This thesis is a product of a significant amount of time spent on boats. I especially thank Willy Hatch and the M/V Machaca, fishermen in the Shark's Eye Tournament in Montauk, New York, Chris Fischer and the M/V OCEARCH, Eric Savetsky, Tom Burns, Wayne Davis and the $\mathrm{M} / \mathrm{V}$ Endurance, the R/V Pelican and the many other men, women and vessels for assistance in field tagging operations including Jason Jaacks, Max Kaplan, Ben Galuardi, Jesse Cochran, Tane Sinclair-Taylor, Will Oestreich, Ben Jones, Julie Cavin, Big Al, and Don Braun. Without your tireless support and strong grip on the tail rope, this work would not have been possible.

Thank you to the WHOI Academic Programs and MIT Joint Program staff for your support and dedication to making things run smoothly. Also a major thank you to Drew Brown for his never-ending patience, Becky Gast for her support as education coordinator throughout my time in the JP and the WHOI Biology Department and greater WHOI community for letting me call this place home.

I am grateful to my thesis committee, Glenn Flierl, Gareth Lawson, Dennis McGillicuddy and Greg Skomal, for graciously volunteering their time and knowledge to this endeavor and for their valuable feedback along the way. Many thanks to Michael Moore for serving as my thesis chair. I would also like to thank my co-authors: Peter Gaube, Ben Galuardi, Greg Skomal, Pedro Afonso, Jorge Fontes and Tane Sinclair-Taylor. Chi Hin Lam contributed code to several pieces of my work. Thank you, all, for your help.

My advisor, Simon Thorrold, has taught me a great deal about being a scientist and a person. He had the patience and drive to teach and support me from my early days as a summer student to the culmination of my PhD. Without Simon's guidance, I would certainly not be where I am today. He has gone above and beyond his "duty" as an advisor, and I won't soon forget the many fond memories with the Thorrolds.

After nearly eight years of living on the Cape I've been fortunate to cross paths with many amazing people. To my friends, fellow JP students, and many others who I've gotten to know during my time as a student, an enormous thank you for your support and for the memories we shared. Lobster fishing in blizzards, tagging sharks from inflatable boats, eating more amazing meals with incredible people than I can count, scaling the Swiss Alps, skiing post-Soviet Georgia, diving remote tropical atolls and many other memories will stick with me forever.

I am especially grateful for the support from my family who instilled in me a love for being outside and the work ethic that keeps me going. You taught me to be curious about 
the world around me, didn't blink (much) when that curiosity led me to the Red Sea or took me off the grid for months at a time, and have stuck with me despite my love for an incredibly rewarding but seemingly fiscally-irresponsible career. 


\section{Contents}

$\begin{array}{lll}1 & \text { Introduction } & 17\end{array}$

1.1 It's a big ocean: approaches to locating animals . . . . . . . . . . . . . 19

1.2 I tagged some fish, now what? . . . . . . . . . . . . . . . . . . 20

1.3 Why did they do that? . . . . . . . . . . . . . . . . . 21

1.4 Why should we care? . . . . . . . . . . . . . . . . . . . . 22

1.5 Thesis Overview . . . . . . . . . . . . . . . . . . . 23

2 An improved hidden Markov method for geolocating archival-tagged fishes 25

2.1 Summary . . . . . . . . . . . . . . . . . . . 26

2.2 Introduction . . . . . . . . . . . . . . . . . 26

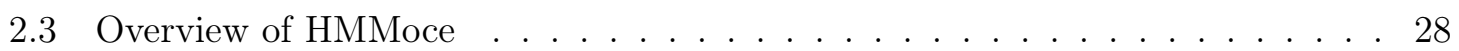

2.3.1 Model formulation . . . . . . . . . . . . . . . . . . 28

2.3.2 Computational improvements and requirements . . . . . . . . . . 31

2.4 Case study: pelagic shark movements . . . . . . . . . . . . . . . 31

2.5 Conclusions . . . . . . . . . . . . . . . . . . . . . 37

3 Integrating archival tag data and a high-resolution oceanographic model to estimate basking shark (Cetorhinus maximus) movements in the western Atlantic $\quad 39$

3.1 Abstract . . . . . . . . . . . . . . . . . . . . 40

3.2 Introduction . . . . . . . . . . . . . . . . . 40

3.3 Methods . . . . . . . . . . . . . . . . . . . . . . 42

3.3.1 Study area and tagging . . . . . . . . . . . . . . . 42

3.3 .2 Tag types . . . . . . . . . . . . . . . . . . . . . . . 43

3.3.3 Geolocation methods . . . . . . . . . . . . . . . . . . 43

3.4 Results . . . . . . . . . . . . . . . . . . 46

3.5 Discussion . . . . . . . . . . . . . . . . . . . . 51

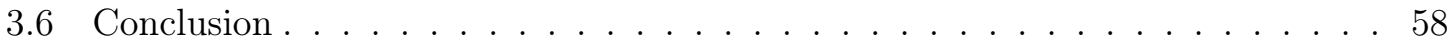

4 Determining oceanographic association, movements and connectivity of swordfish in the North Atlantic by integrating electronic tagging and fisheries data

4.1 Abstract . . . . . . . . . . . . . . . . . . . 62 
4.2 Introduction . . . . . . . . . . . . . . . . . . . . 62

4.3 Materials and methods . . . . . . . . . . . . . . . . . . 64

4.3 .1 Satellite tagging . . . . . . . . . . . . . . 64

4.3 .2 Fisheries data . . . . . . . . . . . . . . . . . . . 6 67

4.3 .3 Oceanographic data . . . . . . . . . . . . . . . . 67

4.3.4 GAM model formulation . . . . . . . . . . . . . . . . . . . . . . . 69

4.4 Results . . . . . . . . . . . . . . . . . . . . . . . 71

4.4 .1 Geolocation of PSATs . . . . . . . . . . . . . . . 71

4.4 .2 Horizontal movements . . . . . . . . . . . . . . . . 71

4.4 .3 Oceanographic association . . . . . . . . . . . . . . 73

4.4.4 Factors influencing CPUE . . . . . . . . . . . . . . . 75

4.5 Discussion . . . . . . . . . . . . . . . . . . 77

4.5.1 Movements and connectivity . . . . . . . . . . . . . . . 77

4.5.2 Oceanographic association . . . . . . . . . . . . . . . . . . 79

4.6 Conclusions . . . . . . . . . . . . . . . . . . . . . . 84

5 Warm core rings release predators from thermal constraints when foraging $\begin{array}{ll}\text { in the ocean Twilight zone } & 87\end{array}$

5.1 Abstract . . . . . . . . . . . . . . . . . . . . 88

5.2 Main Text . . . . . . . . . . . . . . . . . . . . 89

6 Conclusion and Outlook $\quad 97$

6.1 Summary . . . . . . . . . . . . . . . . . . . . . . . 98

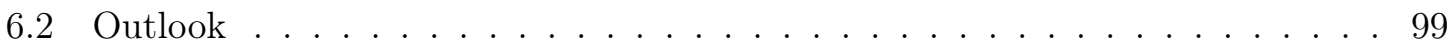

A Chapter 2 Supplemental Information 101

A.1 Supplemental Methods . . . . . . . . . . . . . . . . . . . . . . . . . . . . . . 102

A.1.1 The Approach . . . . . . . . . . . . . . . . . . . . . . . 102

A.1.2 Data preprocessing . . . . . . . . . . . . . . . . . 102

A.1.3 Calculating likelihoods . . . . . . . . . . . . . . . . . . . . . 102

A.1.4 Model formulation . . . . . . . . . . . . . . . . . . . . 105

A.1.5 Case study methods . . . . . . . . . . . . . . . . 107

A.1.6 Other geolocation models . . . . . . . . . . . . . . . . . 108

A.2 Supplemental Figures . . . . . . . . . . . . . . . . . . . . . . . . 110

A.3 Supplemental Tables . . . . . . . . . . . . . . . . . . . . . . . . . 118

B Chapter 4 Supplemental Information $\quad 121$

B.1 Supplemental Figures . . . . . . . . . . . . . . . . . . . . . . . . . 122 
C Chapter 5 Supplemental Information

C.1 Supplemental Methods . . . . . . . . . . . . . . . . . . . . . 128

C.1.1 Satellite tagging and data . . . . . . . . . . . . . . . . . . 128

C.1.2 Oceanographic data . . . . . . . . . . . . . . . . . . . . . . 129

C.1.3 Eddy collocation . . . . . . . . . . . . . . . . . 130

C.1.4 Diving and vertical eddy structure . . . . . . . . . . . . . . . 131

C.2 Supplemental Tables . . . . . . . . . . . . . . . . . . . . . . . . 131

C.3 Supplemental Figures . . . . . . . . . . . . . . . . . . . . . . . . . . 134 
THIS PAGE INTENTIONALLY LEFT BLANK 


\section{List OF Figures}

2-1 Example pop-up satellite archival tag data . . . . . . . . . . . . . . . 29

2-2 Comparison of results from various geolocation approaches . . . . . . . . . . 32

2-3 Movement and behavior results from HMMoce . . . . . . . . . . . . . . . . 35

2-4 Residency distribution results from HMMoce . . . . . . . . . . . . . . 36

3-1 Comparison of depth-temperature profile data . . . . . . . . . . . . . . 46

3-2 Most probable basking shark tracks . . . . . . . . . . . . . . . . . 47

3-3 Basking shark seasonal residency distributions . . . . . . . . . . . . . . . . 51

3-4 Representative basking shark vertical data . . . . . . . . . . . . . . . . . . 52

3-5 Example long-range basking shark movements . . . . . . . . . . . . . . . 53

3-6 Representative basking shark vertical data for long-range movements . . . . . 54

3-7 Basking shark vertical habitat envelopes by season . . . . . . . . . . . . . 55

4-1 Movements of satellite-tagged swordfish in the North Atlantic . . . . . . . . . 70

4-2 Seasonal distribution of catch-per-unit effort of the longline swordfish fishery in the North Atlantic . . . . . . . . . . . . . . . . . . . . . . . . . . . . . . 74

4-3 Movements and connectivity of tagged swordfish in the North Alantic compared to management areas . . . . . . . . . . . . . . 76

4-4 Example use of eddies by an archival-tagged swordfish . . . . . . . . . . . 78

4-5 Collocation of swordfish conventional tag data to anticyclonic and cyclonic eddies across three dynamic regions of the Atlantic . . . . . . . . . . . 80

4-6 Estimated individual effects on swordfish catch per unit effort of environmental covariates in an additive model framework . . . . . . . . . . . . . . . 81

5-1 Use of Gulf Stream eddies by satellite-tagged blue sharks . . . . . . . . . . . . 90

5-2 Eddy vertical structure and blue shark diving . . . . . . . . . . . . . . . . . 92

5-3 Example diving by blue sharks in Gulf Stream eddies and a Sargasso Sea cyclone . . . . . . . . . . . . . . . . . . . . . . 94

A-1 Density of speeds among Argos-based positions for tagged individuals. . . . . 110

A-2 Comparison of track methods for blue shark 141254 . . . . . . . . . . . . . 111

A-3 Comparison of track methods for blue shark 141256 . . . . . . . . . . . . 112

A-4 Comparison of track methods for blue shark 141259 . . . . . . . . . . . . 113

A-5 Example depth-temperature profile-based likelihoods . . . . . . . . . . . 114 
A-6 Calculated HMMoce tracks and behavior for blue shark 141254 . . . . . . . . 115

A-7 Calculated HMMoce tracks and behavior for blue shark 141256 . . . . . . . . . 116

A-8 Calculated HMMoce tracks and behavior for blue shark 141259 . . . . . . . . . 117

B-1 Regression of remotely-sensed SST and model-based SST at a subset of swordfish conventional tag locations . . . . . . . . . . . . . . . . . . . . . . . . 122

B-2 Drifter trajectories used as null movements in the eddy collocation analysis . 123

B-3 Generalized additive model diagnostics for swordfish catch-per-unit effort . . . 124

B-4 Monthly latitude density distribution of swordfish tagged with conventional

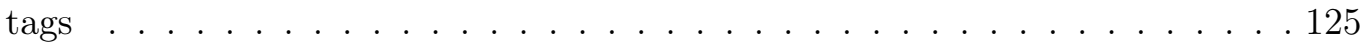

B-5 Distribution of SST and chlorophyll concentrations experienced by satellite and conventional-tagged swordfish in the North Atlantic . . . . . . . . . 126

C-1 Frequency of depth-temperature profile measurements recorded by PSATtagged blue sharks . . . . . . . . . . . . . . . . . . . . . . 134

C-2 Depth-temperature composite and anomaly for Gulf Stream eddies . . . . . . 135 


\section{LIST OF TABLES}

2-1 Satellite tagging summary for HMMoce model data . . . . . . . . . . . . 28

2-2 Validation metrics for geolocation methods . . . . . . . . . . . . . 33

3-1 Summary information from satellite tag deployments on Cetorhinus maximus in the NW Atlantic. . . . . . . . . . . . . . . . . . . . . . . . . . . 49

3-2 Vertical habitat envelope summary statistics . . . . . . . . . . . . . 57

4-1 Summary of swordfish tagged with PSATs in the North Atlantic Ocean . . . . 66

A-1 Example error metrics for HMMoce model runs using different observation likelihoods . . . . . . . . . . . . . . . . . . . . . . . 118

A-2 HMMoce model fit metrics, grid resolution and computation time . . . . . . . 119

C-1 Tagging summary for SPOT and PSAT-tagged blue sharks in the Gulf Stream 132

C-2 Summary of anticyclonic and cyclonic eddy use by blue sharks in the Gulf Stream . . . . . . . . . . . . . . . . . . . . . . 133 
THIS PAGE INTENTIONALLY LEFT BLANK 
Chapter 1

\section{INTRODUCTION}


Marine ecology, particularly of the open ocean, is the study of marine organisms in the context of each other and the environment. The pelagic ocean is characterized by a range of temporal and spatial scales that are manifested as a complex integration of physical, chemical and biological phenomena. The interactions of these dynamics ultimately generate the behaviors we observe from marine animals, yet the scales at which we typically observe animal movements are not concurrent with many important oceanographic features. For example, we often observe movements of fishes at basin scales, revealing fascinating long-distance migrations, or at very small scales when they interact with fishing activities. Yet, perhaps the most important biophysical interactions occur at and below the oceanic (sub)mesoscale with spatial scales of $\mathcal{O}(1-100 \mathrm{~s} \mathrm{~km})$ and time scales of days to months, most of which is poorly resolved in current approaches to studying movements of marine fishes. This lack of knowledge stems from issues inherent in studying animal movements in an opaque medium (such as seawater), particularly with constraints on geolocation of individual fish.

Since the first tag-recapture experiments on Atlantic salmon in 1873 (Everhart, 1975), scientists have been actively studying animal movements. Where animals go and what they do there is of fundamental importance for a range of ocean properties and dynamics, from carbon export (e.g. Lavery et al., 2010) to ecosystem structure (e.g. Thorrold et al., 2014) and fisheries (e.g. Block et al., 2005). Yet, despite significant advances in tag technology, our current understanding of large pelagic fish ecology remains limited primarily to large-scale movements and summarized behavior (Braun et al., 2015a). The accuracy of light geolocation $\left( \pm 100-200 \mathrm{~km} ; 10,000 \mathrm{~km}^{2}\right)$ and its depth limits $(<100 \mathrm{~m})$ constrain its application to large-scale surface movements (Braun et al., 2015b). Yet, many fishes exhibit diving behavior that renders light geolocation impossible (Dewar et al., 2011), and the magnitude of error precludes our ability to resolve important ecological dynamics such as habitat association (e.g. specific bathymetric or oceanographic feature use). As such, studying animals that move thousands of kilometers while diving from the surface to mesoor bathypelagic depths all while immersed in an opaque medium like seawater remains a formidable challenge. Therefore, improved analytical techniques and different approaches to tracking studies are necessary to acquire more accurate position information with which we can characterize the ocean environment and provide context for the animal behaviors we observe. Thus, I focus on the following questions in this thesis:

1. How can we improve current methods of geolocation within the constraints of existing archival tag technology?

2. Can we use improved methods to leverage historical data for use in modern physicalbiological interaction studies, and, by doing so, improve the inference we can gain from this data? 
3. How can we apply the lessons learned from the first two questions to effectively quantify oceanographic associations of large pelagic fishes?

\subsection{It's a big ocean: approaches to locating animals}

The study of marine animal movements remains a formidable challenge due to the difficulty of making observations on elusive, highly migratory species in a vast ocean. Information on large pelagic fishes in the open ocean is particularly lacking, despite lucrative fisheries and intense fishing effort for a number of these species. Basin-scale movements (Skomal et al., 2009) and deep forays to meso- and bathypelagic depths (Thorrold et al., 2014) further complicate research efforts by making individuals even less available for direct observations than coastal fish species or terrestrial taxa.

Historically, insights into pelagic fish ecology were limited to those obtained visually at the surface. These constraints allowed access to only a small snapshot of any species' ecology, and it has long been recognized that novel approaches are needed to better understand these species. Mark-recapture techniques employing external tags have been used extensively since the early twentieth century, but this approach only provides deployment and retrieval locations with no data on a fish's behavior in the interim (Kohler and Turner, 2001).

The development of electronic tags in the mid-1950s has since enabled researchers to gather a more holistic view of the behaviors of large pelagic organisms. Temperature and pressure sensors were developed simultaneously in the 1970s and transformed our ability to follow the incredible dives that many species perform (Carey and Robison, 1981). Satellite transmitting tags followed in the $80 \mathrm{~s}$ and allowed accurate positioning of surface-oriented species such as basking sharks (Priede, 1984). Data storage, or archival, tags were first deployed in the 1990s and have since been widely used worldwide (Hussey et al., 2015). Modern tags can incorporate multiple sensors (e.g. echosounders, accelerometers and cameras) and can release themselves from a study animal, effectively eliminating the fishery-dependent nature of earlier studies. While other approaches exist, the two most widely used electronic tagging technologies in aquatic ecosystems today are acoustic and satellite-based tags (Hussey et al., 2015).

Acoustic techniques proliferated starting in the 1960s and have since made many significant contributions to the field (e.g. Carey and Robison, 1981; Carey and Scharold, 1990). These techniques rely on transmission of acoustic signals by tagged animals that are logged at moored or mobile receiving stations. Acoustic tags may now last up to 10 years, typically exhibit error on the order of meters and, in some cases, can leverage satellite-linked loggers that eliminate the need to recover devices to access stored data (Donaldson et al., 2014). The benefits of acoustic telemetry have driven exceptional growth in acoustic monitoring (Hussey et al., 2015); however, current acoustic studies are limited to tag detection within $<$ 
$2 \mathrm{~km}$ range from receiving stations (e.g. hydrophones) which proves intractable for studying highly migratory species in the open ocean. Thus, the major limitation in acoustic studies is scale yet understanding how observed quantities scale in the ocean is perhaps among the most interesting ocean challenges (Stommel, 1963; Haury et al., 1978).

In the late 1980s, satellite-linked archival tags followed the advent of acoustic devices. This technology alleviated the in situ infrastructure, and thus scaling, issues associated with acoustic telemetry, enabling researchers to follow fish that move thousands of kilometers across ocean basins in a matter of months, such as tunas (Block et al., 2005) and pelagic fishes and sharks (Block et al., 2011). Since their inception, these tags have become increasingly relevant (Hussey et al., 2015) for studying horizontal and vertical movements (Block et al., 2011; Thorrold et al., 2014; Berumen et al., 2014), residency (Domeier, 2006), mortality (Musyl et al., 2011), aggregative and feeding behaviors (Jorgensen et al., 2012), and other aspects of the biology and ecology of marine organisms. Pop-up satellite archival transmitting (PSAT) tags, specifically, have been used with great success on a number of taxa. These devices are attached externally to a study animal and are programmed to pop-up from the individual after a predetermined deployment period. While active on the animal, these tags typically collect an in situ time series of depth, temperature and light levels. Miniaturization and ever-improving sensors, batteries, storage capabilities, fisheries independence and other advantages make PSATs a popular tool, and this technology has been deployed on thousands of study animals encompassing nearly all marine taxa large enough to carry a tag (Hussey et al., 2015). Thus, satellite tag studies have now described the broad-scale horizontal and vertical movements for many marine species. However, fewer

studies attempt to perform quantitative analysis of how species interact with and choose to occupy the surrounding oceanographic environment (except see, for example, Lawson et al., 2010), primarily due to constraints imposed by geolocation accuracy.

\subsection{I tagged some fish, now what?}

Despite many technological advances, we remain limited by the fundamental physics of transmitting electromagnetic radiation (the way we communicate with satellites such as GPS) through an opaque medium such as seawater. As such, even cutting edge satellitebased tags for studying fish still archive light data for geolocation. Ambient light records are used to estimate dawn and dusk from which longitude (local noon) and latitude (local day length) are derived to estimate positions (Hill, 1994; Hill and Braun, 2001). Early on, the community recognized the need to supplement light-based geolocation with other information, and we have since made sizable advances in analytic approaches to improved geolocation despite the age-old constraints.

Arguably the most important advances in the analysis of animal movement data, in- 
cluding the geolocation problem, has been state-space models which estimate the "state" of an unobserved process from an observed dataset (Jonsen et al., 2013). The most notable early advance was the application of the Kalman filter (that has applications from natural sciences to economics) by Sibert and Fournier (2001) to analyze position estimates from noisy light data (Hill and Braun, 2001). Further advances were quickly achieved by incorporating a comparison of remotely-sensed sea surface temperature (SST) with in situ measurements onboard the tag (Teo et al., 2004; Nielsen et al., 2006). Improvements since have expanded rapidly to incorporate other ancillary data such as tidal variation (Metcalfe and Arnold, 1997) to inform geolocation and more sophisticated modelling techniques such as hidden Markov models (Pedersen et al., 2008). The integration of in situ measurements onboard archival tags with ever-improving statistical techniques and modern tools such as high-resolution oceanographic models and a suite of remote-sensing satellites promises to yield significant improvements in estimating animal movements over geolocation with light levels alone.

\subsection{Why did they do that?}

Of particular relevance to this thesis is the role that oceanography plays in the structuring of pelagic ecosystems. Fronts and mesoscale eddies are among the most important features in the open ocean (Chelton et al., 2011; McGillicuddy, 2016; Mahadevan, 2016), and recent advances in satellite oceanography have allowed the automated identification and tracking of these features globally (Chelton et al., 2011; Belkin and O'Reilly, 2009). Advances in our ability to observe and track these features have revealed rich regional variability in how they influence lower trophic levels (McGillicuddy, 2016; Gaube and McGillicuddy, 2017) and have shown the potential coupling of biology and ocean physics that can lead to the formation of biological hotspots (Mann and Lazier, 2006; Belkin et al., 2014).

Electronic tag technologies permit quantitative analyses of the use of these features by pelagic predators. However, due to geolocation constraints for fishes (see Section 1.2), the vast majority of advances have been made on obligate surface-oriented taxa such as turtles (Gaube et al., 2017; Polovina et al., 2006; Kobayashi et al., 2011), marine mammals (Johnston and Read, 2007; Bailleul et al., 2010) and birds (Thorne and Read, 2013; Tew Kai et al., 2009). Together, these studies suggest the most important features for pelagic predators are associated with enhanced vertical flux of nutrients leading to increases in primary production (Franks, 1992). Convergent flow at front boundaries and along the periphery of eddies can also aggregate passive particles, including phytoplankton, and these areas are thought to attract pelagic predators due to increased foraging opportunities (Scales et al., 2014).

Historically, anecdotal evidence and fisheries statistics have also supported the associa- 
tion of pelagic fishes with physical structures like fronts and eddies (e.g. Hobday and Hartog, 2014), but scientists currently understand very little about the biology of these important oceanographic features, particularly for fish communities. Technological limitations inherent in light-level geolocation (Braun et al., 2015b) have largely precluded robust analyses of the associations between (sub)mesoscale oceanographic structures and pelagic fishes. As a result, we understand little about the biophysical factors influencing the ecology of large pelagic fishes. Despite these constraints, some progress has been made, primarily using fisheries data. For example, Hsu et al. (2015) compared catch data from the U.S. northwest Atlantic longline fishery to the mesoscale eddy field in this region and found bluefin tuna associated with anticyclonic mesoscale eddies while swordfish were more often outside of eddies. Similarly, using catch data, Hobday and Hartog (2014) found southern bluefin tuna associated with anticyclones off Australia while opah preferred cyclonic eddies. Similar analyses have been conducted using other fisheries, species and ocean features, such as fronts (e.g. Worm, 2005).

A few recent studies have used tracking data to investigate overlap between pelagic fishes and ocean features. For example, Miller et al. (2015) showed basking sharks prefer productive regions of the Northeast Atlantic characterized by contemporaneous thermal and chlorophyll fronts. And Gaube et al. (2018) showed two tagged white sharks associated with anticyclonic eddies of the Northwest Atlantic, suggesting these features may influence foraging opportunities. However, analyzing fisheries-independent tracking data in the context of (sub)mesoscale oceanography is in its infancy, particularly for fishes. In addition, few studies of fish movements have been specifically designed to investigate feature use. Thus, linking movements of pelagic fishes with (sub)mesoscale physical-biological mechanisms remains largely unstudied but is critical to understanding the structuring of pelagic ecosystems and to designing appropriate management approaches for pelagic fish populations (Hazen et al., 2018).

\subsection{Why should we care?}

Over the past century, human exploitation of natural systems has propagated throughout the ocean, subjecting large marine predators to intense exploitation (Byrne et al., 2017) and eliciting disproportionate effects on large vertebrates (Jackson et al., 2001; Baum et al., 2003). As a result of intense anthropogenic impact, many predatory fish populations (including billfish, tuna and sharks) have joined the ranks of immediate conservation concern, and their populations are routinely depleted by 50-70\% (Hilborn et al., 2003) and up to 90\% (Myers and Worm, 2005). Many of these species spend most of their life in the open ocean and traverse vast expanses of water in search of food, reproductive opportunities and suitable habitat. Their behavioral tendencies subject them to fishing pressure by an ar- 
ray of different gear types and exploitation levels under different national jurisdictions and throughout the high seas. In addition, the current lack of information about key life history traits, population size, movements and habitat use of these fishes amplifies the problem of managing these populations as anthropogenic pressures on fishes continue to rise (Dulvy et al., 2008; Ferretti et al., 2010).

A central challenge to the management of ocean seascapes is the dynamic spatial and temporal nature of ocean systems (Lewison et al., 2015). Yet, traditional ocean management approaches are most often static. Static management approaches may be less effective for managing highly migratory species and are less able to respond to changing ocean dynamics at scales from ephemeral features, such as eddies, to chronic climate-induced changes. Relatively simple time series of animal movements and behavior coupled with modeled and/or remotely-sensed representations of the marine environment, as described in this thesis, can be combined to develop tools for near real-time prediction of species-specific habitat use (Hazen et al., 2018; Brodie et al., 2018). These dynamic approaches will be critical for managing fisheries in a changing ocean (Maxwell et al., 2015), but necessitate some understanding of physical-biological mechanisms governing observed animal behaviors.

Filling these gaps will be essential for formulating effective management plans (CullisSuzuki and Pauly, 2010), understanding the potential effects of climate change (Hazen et al., 2012) and ensuring continued harvest of these resources (Pauly, 1998; Watson et al., 2013). Additionally, an improved understanding of the behaviors of large pelagic fishes will not only inform us about the ecology of the taxa themselves, but may also facilitate broader understanding of biogeochemical processes in the ocean (Lavery et al., 2010; Roman and McCarthy, 2010).

\subsection{Thesis Overview}

With the aforementioned questions in mind, this thesis seeks to determine the oceanographic associations of large pelagic fishes in the North Atlantic Ocean to better understand species ecology and ecosystem dynamics. In Chapter 2, I developed significant methodological improvements for geolocating fishes equipped with traditional archival tags. Blue and mako sharks were instrumented both with PSATs and an independent Doppler-based satellite tag from which "known" locations were used to quantify error in the resulting PSAT geolocation model. Leveraging three-dimensional data from tags in conjunction with high-resolution oceanographic models facilitated significant improvements in error estimates relative to existing model frameworks. In Chapters 3 and 4, I applied this modeling technique to basking shark and swordfish datasets, respectively. These two species have proven particularly difficult to track due to significant occupation of the aphotic regions of the ocean resulting in little to no light data available for geolocation. In Chapter 3, I used the improved geoloca- 
tion estimates to quantify large-scale movements, seasonality and vertical habitat use of $>$ 50 basking sharks in the western Atlantic. Improved swordfish tracks in Chapter 4 were also used to investigate movements and seasonality and, in some cases, were accurate enough to describe mesoscale feature use. I also mined fisheries-dependent data for swordfish in the North Atlantic in Chapter 4 that I synthesized with fishery-independent electronic tag data to quantify oceanographic associations of swordfish. Finally, in Chapter 5 I constructed a robust dataset with which I could test interactions between pelagic predators and mesoscale eddies. I double-tagged blue sharks with PSATs and satellite-based positioning tags (as in Chapter 2 above) in order to reconstruct 3-D movements in the Gulf Stream eddy field. I collocated these data to remotely-sensed and modeled oceanographic data to quantify shark-eddy interactions. Overall, in this thesis I demonstrate that integrating observations of animal movement and behavior with satellite imagery and physical data provides significantly enhanced insight on habitat preferences and the physical-biological interactions between fishes and the marine environment. 
Chapter 2

\section{AN IMPROVED HIDDEN MARKOV METHOD FOR GEOLOCATING ARCHIVAL-TAGGED FISHES}

This chapter was originally published as Braun, C.D., Galuardi, B., and Thorrold S.R. (2018). HMMoce: An $\mathrm{R}$ package for improved geolocation of archival-tagged fishes using a hidden Markov method. Methods in Ecology and Evolution 9, 1212-1220.

C.D.B and B.G. conceived the project and developed the package; C.D.B and S.R.T. collected the data; C.D.B. wrote the paper; B.G. and S.R.T. contributed to the writing of the paper.

The supplemental methods, figures, and tables for this chapter can be found in Appendix A. 


\section{$2.1 \quad$ Summary}

1. Electronic tagging of marine fishes is commonly achieved with archival tags that rely on light levels and sea surface temperatures to retrospectively estimate movements. However, methodological issues associated with light-level geolocation have constrained meaningful inference to species where it is possible to accurately estimate time of sunrise and sunset. Most studies have largely ignored the oceanographic profiles collected by the tag as a potential way to refine light-level geolocation estimates.

2. Open-source oceanographic measurements and outputs from high-resolution models are increasingly available and accessible. Temperature and depth profiles recorded by electronic tags can be integrated with these empirical data and model outputs to construct likelihoods and improve geolocation estimates.

3. The $\mathrm{R}$ package HMMoce leverages available tag and oceanographic data to improve position estimates derived from electronic tags using a hidden Markov approach. We illustrate the use of the model and test its performance using example blue and mako shark archival tag data. Model results were validated using independent, known tracks and compared to results from other geolocation approaches.

4. HMMoce exhibited as much as 6-fold improvement in pointwise error as compared to traditional light-level geolocation approaches. The results demonstrated the general applicability of HMMoce to marine animals, particularly those that do not frequent surface waters during crepuscular periods.

\subsection{Introduction}

Electronic archival tags have been widely adopted by ecologists to track movements of wideranging species that are difficult to monitor using other techniques. In ocean environments, implanted archival and pop-up satellite archival transmitting (PSAT) tags have proved particularly valuable in the study of life history patterns (e.g. Thorrold et al., 2014), biophysical interactions and habitat use (e.g. Braun et al., 2015b; Lam et al., 2014), horizontal and vertical movements (e.g. Braun et al., 2014; Lam et al., 2016; Werry et al., 2014), and the spatial structure of populations (Skomal et al., 2009; Galuardi et al., 2010; Galuardi and Lam, 2014) in a number of commercially important fishes (Block et al., 2011) and species of conservation concern (Braun et al., 2015a). Yet, tracks provided by electronic tags that rely on light-level geolocation often exhibit large error in daily position estimates (Musyl et al., 2011; Braun et al., 2015b) that may hinder inferences drawn from the tag data. Approaches that provide more certainty in movement estimates derived from light level data (Galuardi and Lam, 2014; Luo et al., 2015) would increase the power of ecological hypotheses tested using tag data. 
Electronic archival tags typically use light levels to estimate position when it is not possible for the tag to interrogate geo-location satellites (Sibert et al., 2003; Nielsen and Sibert, 2007). Accuracy of geolocation using light levels, however, is limited ( $\pm 100-200 \mathrm{~km}$; $\sim 10,000 \mathrm{~km}^{2}$ ) even for surface-oriented species where good light data is available (Wilson et al., 2007; Braun et al., 2015b). While several studies have incorporated ancillary data, including sea surface temperature (Smith and Goodman, 1986; Lam et al., 2010), tidal fluctuation (Pedersen et al., 2008) or ocean heat content (Luo et al., 2015) to help improve geolocation estimates, only one used all data collected from archival tags within a rigorous statistical framework to improve geolocation estimates (Sumner et al., 2009). Although excursions from the photic zone, including diel vertical migration (Neilson et al., 2009) and extended mesopelagic occupation (Skomal et al., 2009) may render light geolocation impossible, the depth-temperature profiles recorded by the tags provide diagnostic oceanographic signatures that can be leveraged to help constrain position (Skomal et al., 2009; Aarestrup et al., 2009).

Hidden Markov Models (HMMs) have gained popularity in recent years as a tool for analyzing animal movement data and have been applied to understand movements of a number of organisms (Holzmann et al., 2006; Thygesen et al., 2009; Pedersen et al., 2011a). Much of the progress in ocean environments stems from a HMM used to track cod in the North Sea using tidal information (Pedersen et al., 2008). The approach combined a number of desirable features, including inference about the underlying behavioral state of the animal, mobilization of oceanographic data in a spatial likelihood framework (Nielsen et al., 2006), and later incorporated formal treatment of barriers to movement (Pedersen et al., 2011a). Generally, the Bayesian HMM approach uses a model of animal movements (e.g. Brownian motion) and a model or observations of the environment (e.g. in situ oceanography) to estimate the posterior distribution of the state (e.g. animal position and behavior). Several $\mathrm{R}$ packages exist for analyzing movement data with HMMs, including ctmm (Calabrese et al., 2016) and moveHMM (Michelot et al., 2016), but none are designed for geolocating marine fishes with archival tag data. An electronic tag manufacturer (Wildlife Computers, Inc.) recently updated their proprietary software (GPE3) to geolocate archival tag data based on light levels and sea surface temperature (SST) in a HMM framework following Pedersen et al. (2008). However, GPE3 is limited by a lack of behavior state-switching dynamics and does not include functionality for non-surface oriented species. GPE3 is also proprietary software that cannot be modified by the user and is limited to tags built by Wildlife Computers.

Our primary objective was to build an analysis toolkit to improve geolocation estimates from electronic archival tags deployed on marine animals that alleviates many of the limitations imposed by previous approaches. The new $R$ package HMMoce uses available electronic tag data and oceanographic data mined from ocean observing system portals to estimate animal movements, behavior, and residency from uncertain and temporally corre- 
lated movement data. We modify and expand a hidden Markov approach (Thygesen et al., 2009; Pedersen et al., 2008, 2011a) that, in addition to estimating animal movements, allows behavior state estimation and provides information about the posterior distribution of the modeled states that can be used as a residency metric (Pedersen et al., 2011a). The modeling framework we developed is sufficiently flexible to accommodate other tag types and animal movement questions, can be applied in any geographic location, and benefits from the transparency of a widely-used open source platform. Here we describe the model framework and demonstrate its applicability using example data. For specific details on package use and functions and a full tutorial with an example dataset, please refer to the package and its accompanying vignette, available on CRAN.

\subsection{Overview of HMMoce}

\subsubsection{Model formulation}

We present a process-based approach to estimate residency and behavior from movement data collected with electronic archival tags. The logic of this approach involves calculating gridded observation likelihoods at each time point based on tag and environmental data, forming the state-space model, estimating model parameters and model selection and interpretation. The application of grids to explicitly resolve space is a key component that allows state estimation (location and behavior, in this case) to be supplemented by or based entirely on environmental data (e.g. temperature at depth). The details of the discretized grid HMM approach are thoroughly explained elsewhere (e.g. Thygesen et al., 2009; Pedersen et al., 2011a). A detailed methodology for our approach can be found in the Appendix A.

Briefly, observation-based likelihoods (Eq. A.1) were derived from in situ SST (Eq. A.2), light-based longitude and depth-temperature profile data (Eq. A.3, A.4, A.5) collected by the tags using five separate likelihood calculations: 1) An SST likelihood (Eq. A.2) was gen-

Table 2-1: Tagging summary for double-tagged blue (BSH) and shortfin mako (MKO) sharks used in this study.

\begin{tabular}{lllllllll}
\hline Species & Tag ID & Start Date & End Date & $\begin{array}{l}\text { Duration } \\
(\mathbf{d})\end{array}$ & $\begin{array}{l}\text { PDT } \\
(\mathbf{\%})\end{array}$ & $\begin{array}{l}\text { Light } \\
\mathbf{( \% )}\end{array}$ & $\begin{array}{l}\text { SST } \\
(\boldsymbol{\%})\end{array}$ & $\begin{array}{l}\text { SPOT } \\
(\mathbf{\%})\end{array}$ \\
\hline BSH & 141254 & $2015-10-21$ & $2016-02-05$ & 107 & 72 & 100 & 92 & 96 \\
BSH & 141256 & $2015-10-13$ & $2016-02-24$ & 134 & 66 & 94 & 88 & 87 \\
BSH & 141259 & $2015-10-13$ & $2016-04-10$ & 180 & 53 & 94 & 82 & 85 \\
MKO & 141257 & $2015-10-15$ & $2016-04-12$ & 180 & 58 & 96 & 69 & 72 \\
\hline
\end{tabular}

PDT, Light, SST and SPOT = percent of deployment period with depth-temperature profile (PDT), light and sea surface temperature (SST) data from the PSAT tag and percent of deployment period with Argos-based positions (SPOT), respectively. 

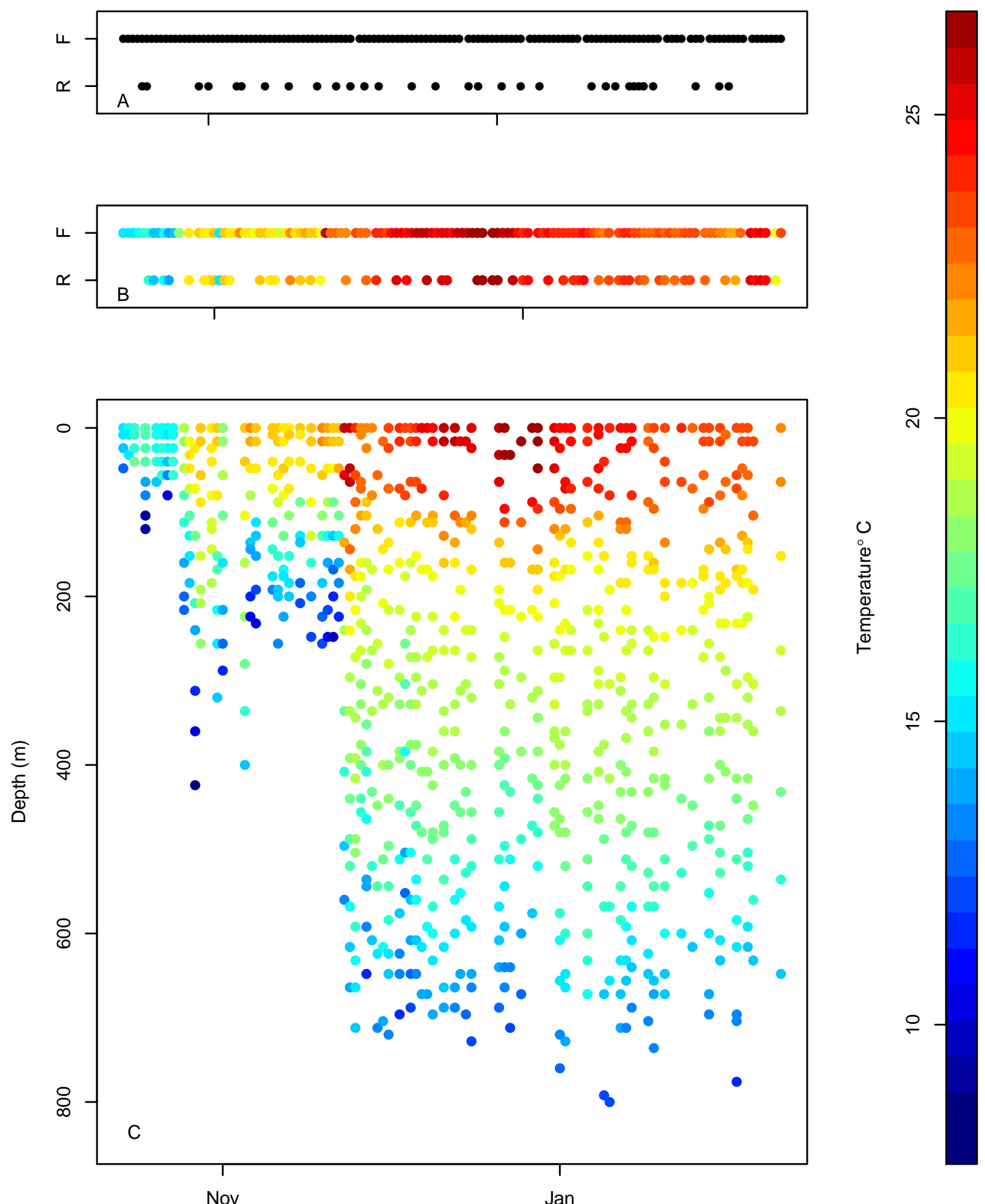

Figure 2-1: Example blue shark data demonstrating the deployment days with (A) light, (B) sea surface temperature and $(\mathrm{C})$ depth-temperature profile data used as the observation portion of the HMM. Full $[\mathrm{F}]$ and removal $[\mathrm{R}]$ datasets for light and SST are shown $(\mathrm{A}, \mathrm{B})$. 
erated for tag-based SST values integrated according to an error term $( \pm 1 \%$, based on tag sensor accuracy) and compared to remotely-sensed SST from daily, optimally-interpolated SST fields (OI-SST, $0.25^{\circ}$ resolution; Banzon et al., 2016). 2) Light-based longitude likelihood was derived using estimates of longitude from GPE2 software (Wildlife Computers, Inc.), which facilitated visual checking of light curves. Depth-temperature profiles recorded by the tag were compared to 3) monthly climatological mean depth-temperature data from the World Ocean Atlas 2013 (WOA, 0.25 resolution; Locarnini et al., 2013) and 4) daily reanalysis model depth-temperature products from the HYbrid Coordinate Ocean Model (HYCOM, $0.08^{\circ}$ resolution; Chassignet et al., 2007) at standard depth levels available in these products (Eq. A.5). Individual likelihood surfaces for each depth level were then combined for an overall profile likelihood at that time point (Eq. A.6). 5) Ocean Heat Content $(\mathrm{OHC})(\mathrm{Eq}$. A.5) was obtained by integrating the heat content of the water column above the minimum daily temperature recorded by the tag for both the tag profiles and HYCOM fields (Eq. A.4; Luo et al., 2015). Start and end locations were considered known in all cases and model runs.

The resulting observation likelihoods (in various combinations; Eq. A.1) were used in a two-step Bayesian state-space approach to estimate the posterior distribution of the state (in this case, a joint probability distribution of location and behavior at each time point). Probability distributions were first calculated forward in time using alternating time and data updates of the current state estimate using a HMM filter (for a detailed methodology of the HMM filter see Appendix 2 in Pedersen et al., 2011a). The filter recursions also returned a likelihood measure indicating how well the model fit the data, which facilitated calculating model parameters (e.g. behavior state-switching probabilities). In Bayesian statistics, the maximum a priori (MAP) estimate of the model parameters is typically used to calculate the posteriors; however, in practice, ample a priori information is rarely available and maximum likelihood (ML) estimates are often very similar to MAP estimates (Jonsen et al., 2005). Thus, we implemented recent advances by Woillez et al. (2016) that further exploited the discretization of space in this model by employing a joint ML estimation of all model parameters using an iterative Expectation-Maximization framework.

Model selection in this context would typically use Bayesian Information criterion (BIC), but this approach requires approximation that imposes restrictions on the priors. Instead, we used Akaike's Information criterion (AIC) for model selection following Pedersen et al. (2011a). The HMM smoother recursion was the final step that worked backwards in time using filtered state estimates and all available observation data to determine smoothed state estimates. This step provided the time marginal of the probability distributions based on observations (posterior distributions).

Results from the final smoothing step represent the posterior distribution of each state over time. Distributions are summed for each behavior state and time step to determine 
the most likely behavior state through time. HMMoce can calculate the mean or mode of the posterior distribution grid, at each time step, to estimate the animal's position. The posteriors can be further analyzed for additional inference including uncertainty and residency. A residency distribution (RD) is conceptually similar to the utilization distribution (UD), but the concept of UD (and other space-use metrics) is often vaguely defined (Royle and Dorazio, 2008). In this case, $\mathrm{RD}$ is interpreted as the estimate of the time spent in a given space within a time interval (see Eq. 5 in Pedersen et al., 2011a).

\subsubsection{Computational improvements and requirements}

While the basic framework of HMMoce was based on previous work (Pedersen et al., 2008; Thygesen et al., 2009; Pedersen et al., 2011a), several improvements were made to accommodate user needs. We focused several enhancements on improving computation efficiency, which was a limitation of previous techniques (SPHMM code for R; Pedersen et al., 2011a). Image processing routines replaced sparse matrix convolution yielding orders of magnitude improvements in computation time, particularly for large, high-resolution grids that characterize geolocation approaches for highly migratory species. In addition, all likelihood routines (except simple light-based likelihood calculations) were parallelized, yielding marked performance improvements, particularly for likelihoods comparing 3D depth-temperature profiles to high-resolution 3D HYCOM grids.

Despite these improvements, HMMoce remains relatively computationally intensive; however, cloud computing is becoming more inexpensive and accessible to a broad user group. The HMMoce package includes a vignette demonstrating simple plug and play functionality for the model using Amazon Web Services's computational resources and an associated machine image containing RStudio Server and all the required dependencies for running HMMoce with user-provided tag data.

\subsection{Case study: pelagic shark movements}

To illustrate the application of HMMoce, we analyzed tag data from three blue sharks (Prionace glauca) and one shortfin mako (Isurus oxyrinchus) that were double-tagged with satellite-linked radio telemetry tags (Wildlife Computers finmount SPOT5 tags) and PSAT tags (Table 2-1). Full tagging methods are provided in Appendix A. We considered the resulting Argos-based tracks as "known" because errors on geolocation estimates from the SPOT tags are much lower (typically $<10 \mathrm{~km}$; Witt et al., 2010; Patterson et al., 2010) than PSAT-based outputs (> $50 \mathrm{~km}$; Winship et al., 2012). The PSAT tags were deployed for an average of 150 days (range 107-180) in the northwest Atlantic with overall movements of 5,403-12,122 km. The PSAT data contained depth-temperature profiles for 53-72\% of days at liberty and SPOT locations were recorded for 72-96\% of deployment days (Table 2-1) 

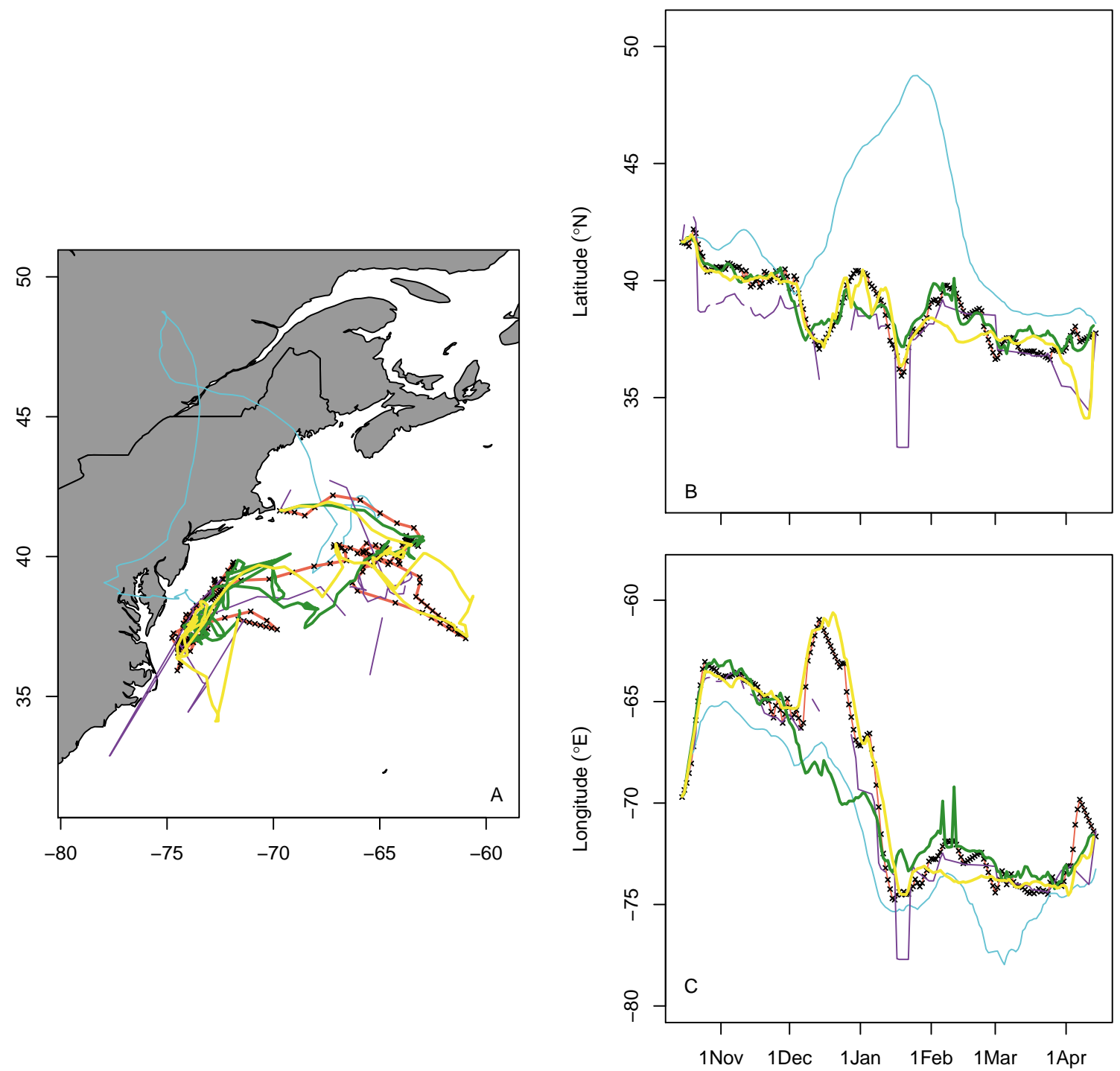

Figure 2-2: Calculated tracks for mako shark 141257 using the 4 different geolocation approaches (Ukfsst, purple; Trackit, blue; GPE3, green; HMMoce, yellow) compared to the "known" Argos-based track (red, black crosses). Latitudinal and longitudinal estimates through time are shown in panels $\mathrm{B}$ and $\mathrm{C}$, respectively. Lines appear broken when a resulting track is missing daily data. 
Table 2-2: Validation metrics for double-tagged blue (BSH) and shortfin mako (MKO) shark tracks estimated using HMMoce, GPE3, Trackit (TI) and Ukfsst.

\begin{tabular}{|c|c|c|c|c|c|c|c|}
\hline Species & Tag ID & Type & Mean (SD) & Median & Range & RMSE & Input \\
\hline \multirow[t]{6}{*}{$\mathrm{BSH}$} & 141254 & HMMoce & $117.4(96.7)$ & 92.4 & $0.5-443.6$ & $1.21,0.81$ & LSO \\
\hline & & GPE3 & $175.8(117.1)$ & 164.3 & $3.2-424.7$ & $1.4,1.64$ & LS \\
\hline & & $\mathrm{TI}$ & $162.3(71.6)$ & 158.2 & $1-328.2$ & $0.97,1.65$ & $\mathrm{~L}$ \\
\hline & & $\mathrm{KF}$ & $179.5(99.5)$ & 178.5 & $1-435.2$ & $1.29,1.24$ & $\mathrm{~L}$ \\
\hline & & HMMoce.r & $131.2(96.2)$ & 101.9 & $0.5-440.5$ & $1.23,1.01$ & LS \\
\hline & & GPE3.r & $157.6(100.6)$ & 143.5 & $1.4-408.9$ & $1.25,1.44$ & LS \\
\hline \multirow[t]{6}{*}{$\mathrm{BSH}$} & 141256 & HMMoce & $83.8(63)$ & 63.7 & $4.9-297.4$ & $0.52,0.93$ & LSH \\
\hline & & GPE3 & $84.9(68.8)$ & 66.9 & $5.9-345$ & $0.66,0.89$ & $\mathrm{LS}$ \\
\hline & & $\mathrm{TI}$ & $474.2(244.1)$ & 459.9 & $0-854.3$ & $1.98,4.84$ & $\mathrm{~L}$ \\
\hline & & $\mathrm{KF}$ & $192.7(152.4)$ & 172.6 & 0-699.8 & $1.35,0.65$ & $\mathrm{~L}$ \\
\hline & & HMMoce.r & $93.4(57.8)$ & 79.1 & $4.2-286$ & $0.59,0.92$ & LSH \\
\hline & & GPE3.r & $423.5(432)$ & 197.8 & $2.1-1394$ & $4.25,3.96$ & LS \\
\hline \multirow[t]{6}{*}{$\mathrm{BSH}$} & 141259 & HMMoce & $179.4(126)$ & 150.3 & $4.4-575.2$ & $1.79,1$ & $\mathrm{LS}$ \\
\hline & & GPE3 & $158.1(109.6)$ & 139.5 & $4.9-434.5$ & $1.44,1.17$ & LS \\
\hline & & $\mathrm{TI}$ & $367.5(239.1)$ & 291.4 & $2.4-861.5$ & $3.3,2.36$ & $\mathrm{~L}$ \\
\hline & & $\mathrm{KF}$ & $245.8(225.5)$ & 194.5 & $1.7-1078.7$ & $2.31,0.88$ & $\mathrm{~L}$ \\
\hline & & HMMoce.r & $183.3(132.2)$ & 140.5 & $4.4-560.5$ & $1.9,0.88$ & LS \\
\hline & & GPE3.r & $198(129.5)$ & 162.0 & $6.1-625.8$ & $1.61,1.77$ & LS \\
\hline \multirow[t]{6}{*}{$\mathrm{MKO}$} & 141257 & HMMoce & $99.8(90.7)$ & 66.8 & $3.8-426.9$ & $0.92,0.99$ & LSH \\
\hline & & GPE3 & 151.1(161.1) & 93.0 & $6.8-675.2$ & $0.65,2.38$ & LS \\
\hline & & $\mathrm{TI}$ & $462.6(347.7)$ & 320.5 & $0-1332.7$ & $4.6,2.79$ & $\mathrm{~L}$ \\
\hline & & $\mathrm{KF}$ & $220.4(151.2)$ & 173.7 & $0-614.6$ & $1.3,1.32$ & $\mathrm{~L}$ \\
\hline & & HMMoce.r & $157.9(128.2)$ & 119.1 & $3.8-494.4$ & $1.05,1.92$ & LSH \\
\hline & & GPE3.r & $182.3(171.8)$ & 136.4 & $0.3-711.2$ & $0.88,2.62$ & $\mathrm{LS}$ \\
\hline
\end{tabular}

Reported error values (mean, sd, median, range) are pointwise distance calculations (mean great circle distance) from known positions (km). Root-mean-square errors (RMSE) are Lat, Lon (degrees). HMMoce.r and GPE3.r indicate fit metrics for data removal experiments in which $75 \%$ of daily light and $50 \%$ of daily SST data was randomly removed. Input indicates input data type: light (L), SST (S), ocean heat content (O), World Ocean Atlas profiles (W) and HYCOM profiles (H). All runs were performed on a $0.08^{\circ}$ grid with fixed migratory speed of $2 \mathrm{~m} \cdot \mathrm{s}^{-1}$ (except 141259 used $\left.4 \mathrm{~m} \cdot \mathrm{s}^{-1}\right)$.

Blue sharks made frequent dives to the mesopelagic zone ( 600-800 m, max 680-1,688 $\mathrm{m})$ but also frequented the surface-air interface where the PSAT tags collected good quality light and SST data (94-100\% deployment days with light, 82-92\% SST)(Figure 2-1). The mako occupied a restricted area ( $\sim 200 \mathrm{~km}$ latitudinal distance) near Cape Hatteras during the winter months and spent relatively little time far from the edge of the continental shelf compared to the more nomadic blue sharks. The mako also had high quality light and SST data (96\% and 69\%, respectively) while regularly diving shallower than the blue sharks $(\sim 400 \mathrm{~m})$. Consistent exposure of the dorsal fin allowed the SPOT tag to acquire Argos 
positions throughout the duration of each deployment (Table 2-1).

We calculated movements of the sharks from PSAT tag data using three modeling approaches that are currently available (Ukfsst, Trackit, GPE3) and HMMoce (Appendix A). Results for the mako are shown in the main text (Figure 2-2), and blue shark figures are provided in the supplement (Figure A-2, Figure A-3, Figure A-4). In general, HMMoce and GPE3 produced the most accurate tracks while those from Ukfsst and Trackit were often unrealistic with errors as high as $>1,300 \mathrm{~km}$ (Table 2-2). For 3 of 4 individuals, HMMoce tracks had the lowest pointwise error and correspondingly lowest root-mean-square error (RMSE) values. For the fourth individual (blue shark 141259), the mean error and RMSE in latitude for GPE3 ouput was lower than HMMoce, which had a lower RMSE in longitude. The traditional approaches (light only, Trackit; light and SST, Ukfsst) yielded much larger error than HMMoce in all cases and only one Trackit output (blue shark 141254 without SST) exhibited marginally smaller error than GPE3 (with SST). In 3 of 4 cases, HMMoce demonstrated the best fitting model by leveraging either OHC $(n=1)$ or HYCOM profiles $(\mathrm{n}=2)$ (Table 2-2) in addition to light-based longitude and SST data used in the other geolocation approaches. The movements of blue shark 141259, in which the HMMoce model did not use profile-based observations to build the final estimated track, included time in both dynamic Gulf Stream water and the more homogenous Sargasso Sea. It proved difficult in both areas to match water column profiles recorded by the tag (or integrated $\mathrm{OHC}$ ) with an accurate and constrained position in the climatological (WOA) or model-based (HYCOM) oceanographic data (Figure A-5).

While HMMoce was designed to improve geolocation estimates for all tagged marine organisms, the main impetus for the work was to fulfill a need for improving track estimates in cases where light and SST data were lacking due to minimal surface occupation. We tested the ability of HMMoce to recover accurate tracks with only limited light-level data by randomly removing (using sample in base R, without replacement) $75 \%$ and $50 \%$ of deployment days with adequate light and SST data, respectively, from the shark PSAT data while keeping the depth-temperature profile data for these days. The removals approximated PSAT data quality typical of swordfish tag deployments in the Atlantic Ocean due to crepuscular diving behavior and light avoidance (Braun et al., 2015a; Neilson et al., 2009). The data removal experiment (Figure 2-1) demonstrated the power of incorporating the depth dimension in likelihood calculations when light and/or SST data is poor. In all 4 cases, HMMoce estimated tracks with lower mean error than corresponding GPE3 results (Table 2-2), but model selection favored including depth-temperature profile information in only 2 of 4 final models. Error in the removal experiment for HMMoce was only marginally higher as compared to the full dataset for 3 of 4 individuals (Table 2-2).

Both GPE3 and HMMoce provide estimated residency distributions (RD; a form of utilization distribution) (Pedersen et al., 2011a). However, only HMMoce incorporates a state- 

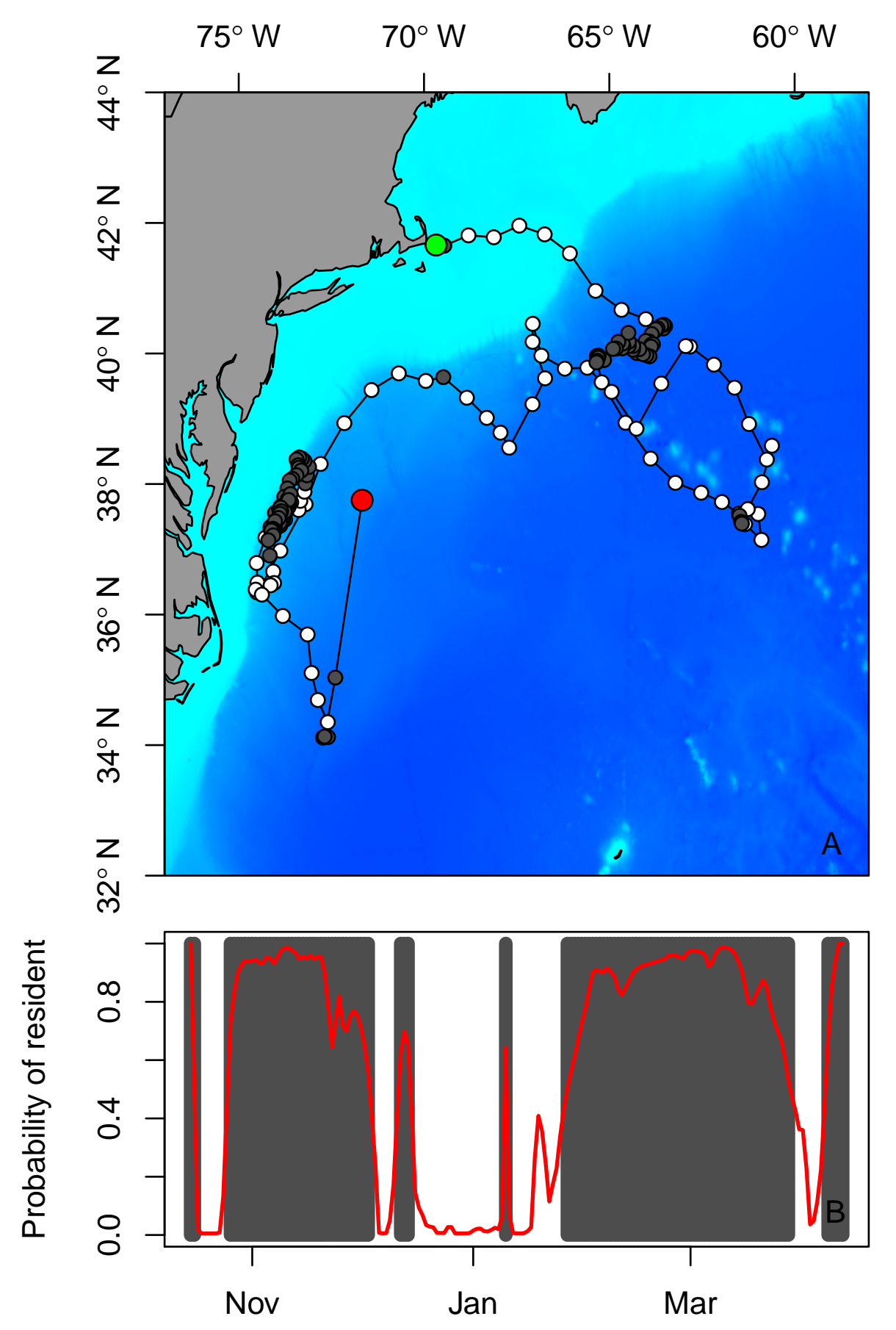

Figure 2-3: Movements (A) and behavior (B) calculated using HMMoce for mako 141257. The tagged individual is considered resident where probability of residency is greater than 0.5 (grey points and bars in panels A and B, respectively). Green and red points indicate tag and pop-up location respectively. Black line follows predicted daily locations of tagged shark. 

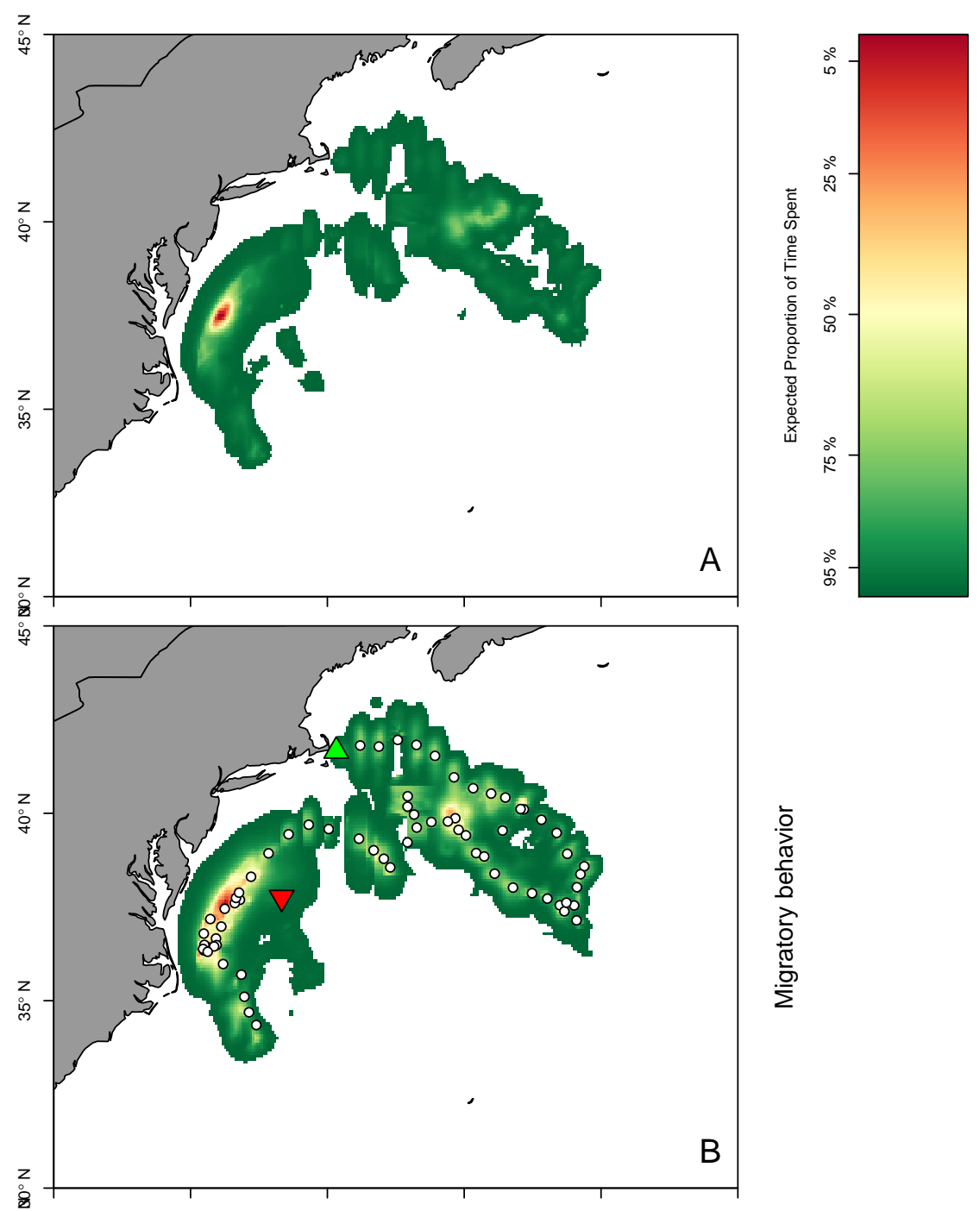

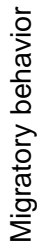

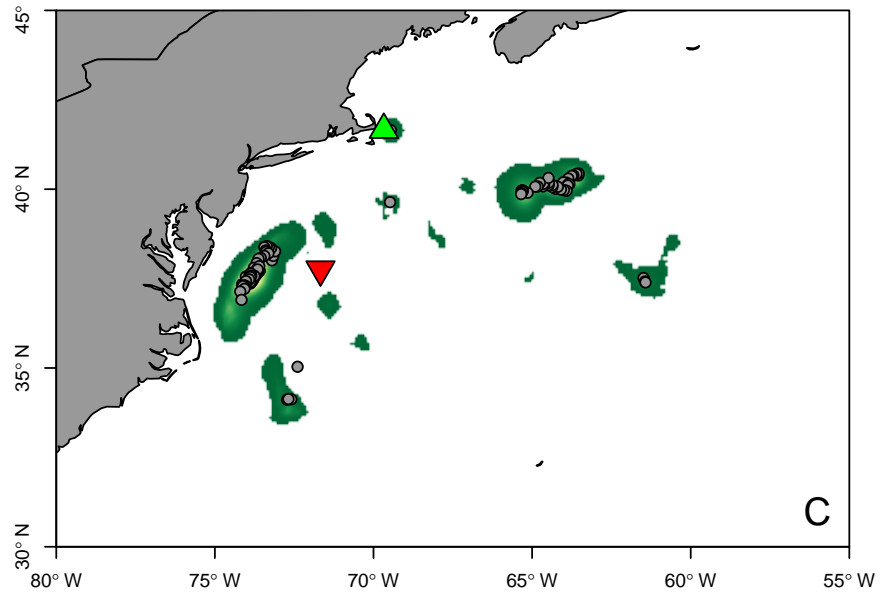

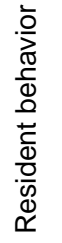

Figure 2-4: Residency distributions for the overall HMMoce modeled movements (A) and for individual behavior states (B, C). Shaded circles indicate residency behavior, white circles indicate migratory behavior, green triangle is tagging location and red triangle is pop-up location. Residency distributions show the expected proportion of time spent in various grid cells over the course of tag deployment. 
switching component that facilitates explicit modeling of distinct animal behaviors (Figure 23). The state-switching is governed by movement kernels (based on speed) and probability of switching between states is calculated by the EM algorithm (Table A-1). For the mako, the RDs indicated areas of core use focused largely where resident behavior was most probable. The RD for the migratory state showed the offshore movement to the SE into the Gulf Stream region before the fish returned to the shelf break and moved SW toward Cape Hatteras. The most notable features of the migratory RD are the overlap areas where the fish transitioned from migratory to resident behaviors (Figure 2-4).

\subsection{Conclusions}

We present a flexible, customizable and transparent HMM framework that may be applied to nearly any marine species utilizing electronic archival tags through a novel use of oceanographic data. Tests of the model demonstrated that HMMoce is a valuable tool for estimating movements from low quality PSAT data through consideration of the vertical structure of the water column in the state estimation. This can be especially beneficial for tag data that is lacking adequate light-level data or other measurements.

For further development, we anticipate several improvements to the HMMoce package. Current priorities include support for other tag types, direct versus derived use of light data, and additional algorithms (e.g. Viterbi) to calculate the most probable track (Pedersen et al., 2011a). Behavior state estimation could be expanded to include advection or modified to update probability with respect to environmental data (Patterson et al., 2009).

We anticipate user feedback will help prioritize further improvements, and we welcome bug reports, feedback, and suggestions for the development of HMMoce via our Github repository https://github.com/camrinbraun/HMMoce. Additional usage information, including an example dataset and a tutorial for using HMMoce on Amazon Web Services, can be found by installing HMMoce in $\mathrm{R}$ (install.packages("HMMoce")) and accessing the package vignette. 
THIS PAGE INTENTIONALLY LEFT BLANK 
Chapter 3

INTEGRATING ARCHIVAL TAG DATA AND A HIGH-RESOLUTION OCEANOGRAPHIC MODEL TO ESTIMATE BASKING SHARK (CETORHINUS MAXIMUS) MOVEMENTS IN THE WESTERN ATLANTIC

This chapter was originally published as Braun, C.D., Skomal, G.B., and Thorrold S.R. (2018). Integrating archival tag data and a high-resolution oceanographic model to estimate basking shark (Cetorhinus maximus) movements in the western Atlantic. Frontiers in Marine Science 5: 25.

G.B.S. and S.R.T. designed the study and conducted the tagging; C.D.B. performed the analysis and wrote the paper. G.B.S. and S.R.T. contributed to the writing of the paper. 


\subsection{Abstract}

Basking shark (Cetorhinus maximus) populations are considered "vulnerable" globally and "endangered" in the northeast Atlantic by the International Union for the Conservation of Nature. Much of our knowledge of this species comes from surface observations in coastal waters, yet recent evidence suggests the majority of their lives may be spent in the deep ocean. Depth preferences of basking sharks have significantly limited movement studies that used pop-up satellite archival transmitting (PSAT) tags as conventional light-based geolocation is impossible for tagged animals that spend significant time below the photic zone. We tagged 57 basking sharks with PSAT tags in the NW Atlantic from 2004-2011. Many individuals spent several months at meso- and bathy-pelagic depths where accurate light-level geolocation was impossible during fall, winter and spring. We applied a newly-developed geolocation approach for the PSAT data by comparing three-dimensional depth-temperature profile data recorded by the tags to modeled in-situ oceanographic data from the highresolution HYbrid Coordinate Ocean Model (HYCOM). Observation-based likelihoods were leveraged within a state-space hidden Markov model (HMM). The combined tracks revealed that basking sharks moved from waters around Cape Cod, MA to as far as the SE coast of Brazil $\left(20^{\circ} \mathrm{S}\right)$, a total distance of over $17,000 \mathrm{~km}$. Moreover, $59 \%$ of tagged individuals with sufficient deployment durations ( $>250$ days) demonstrated seasonal fidelity to Cape Cod and the Gulf of Maine, with one individual returning to within $60 \mathrm{~km}$ of its tagging location one year later. Tagged sharks spent most of their time at epipelagic depths during summer months around Cape Cod and in the Gulf of Maine. During winter months, sharks spent extended periods at depths of at least $600 \mathrm{~m}$ while moving south to the Sargasso Sea, the Caribbean Sea, or the western tropical Atlantic. Our work demonstrates the utility of applying advances in oceanographic modeling to understanding habitat use of highly migratory, often meso- and bathy-pelagic, ocean megafauna. The large-scale movement patterns of tagged sharks highlight the need for international cooperation when designing and implementing conservation strategies to ensure that the species recovers from the historical effects of over-fishing throughout the North Atlantic Ocean.

\subsection{Introduction}

The basking shark, Cetorhinus maximus (Gunnerus 1765), is the second largest fish species, attaining weights of up to 4 tonnes and lengths up to $12 \mathrm{~m}$ (Sims, 2008). It is known to inhabit boreal to tropical (Skomal et al., 2009) waters circumglobally and is most often observed on continental shelves (Sims et al., 2006). Despite its size and widespread distribution, major gaps in our understanding of basking shark ecology remain. Population size and structure are currently unresolved and information about fisheries interactions is 
limited (Sims, 2008). Although there is evidence to suggest population recovery in some areas following exploitation (Witt et al., 2012), the lack of information about key life history traits, population size, movements, and habitat use is problematic as global anthropogenic pressures on elasmobranchs continue to rise (Dulvy et al., 2008; Ferretti et al., 2010).

Basking sharks exhibit life history characteristics that make them particularly vulnerable to exploitation, including low fecundity, slow growth and maturity, and long gestation times (Compagno, 1984; Sims, 2008). There is, therefore, concern over the status of basking shark populations worldwide, and the species is listed on Appendix II of the Convention on the International Trade in Endangered Species (CITES) and Appendices I and II of the Convention for the Conservation of Migratory Species of Wild Animals (CMS). It is also considered "vulnerable" globally and "endangered" in the northeast Atlantic by the International Union for the Conservation of Nature (IUCN).

Historically, information on the ecology of large pelagic animals has been limited to scarce observations that are limited geographically (Templeman, 1963; Squire Jr, 1990; Francis and Duffy, 2002). Almost all of our knowledge of basking shark ecology, for instance, comes from surface observations in coastal waters (Sims et al., 2006; Sims, 2008). Yet recent evidence from electronic archival tags suggests that perhaps the majority of their lives are spent offshore at depths below the euphotic zone (Skomal et al., 2009). Indeed, the rapid development of electronic tag technologies has provided a powerful means of gaining detailed information about the behavior of marine species (Block et al., 2011). PSAT tags have been particularly helpful in ocean environments as data are relayed back to the researcher via satellite upon tag release from the individual (e.g. Block et al., 2011). These tags have provided a wealth of information on sharks (Werry et al., 2014; Berumen et al., 2014), rays (Braun et al., 2014; Thorrold et al., 2014), and large teleost fishes (Braun et al., 2015a) by eliminating the need to physically recover the tag at the end of the deployment.

While electronic tags have revolutionized the study of movement ecology in the ocean, a significant hurdle remains when attempting to track marine fishes compared with terrestrial counterparts. Tags using Argos or Global Positioning System (GPS) locations require the tag antenna to break the water surface long enough for communication with satellites to be established (Argos) or a snapshot of the satellite constellation to be received (GPS). Researchers, have, therefore, relied mostly on PSAT tags that use light-level geolocation in which a threshold algorithm is used to detect solar altitude above the horizon from which estimates of longitude (local noon) and latitude (sunrise/sunset) can be estimated (Hill and Braun, 2001). While sea surface temperature (SST) and bathymetry can improve these estimates (Galuardi et al., 2010; Lam et al., 2010), light-based geolocation requires occupation of the photic zone to record adequate light data for geolocation, and even estimates with quality light data can be error prone (Braun et al., 2015b). However a number of marine species rarely, if ever, experience enough downwelling light or spend adequate time at the 
surface to determine their position with PSAT tags (Aarestrup et al., 2009; Skomal et al., 2009; Peklova et al., 2012). Animals that spend significant time at depths below the photic zone have, therefore, proved extremely difficult to track in ocean ecosystems (e.g. Skomal et al., 2009; Dewar et al., 2011).

The use of PSAT tags to track basking shark movements has proved particularly difficult in the northwestern Atlantic as basking sharks spend months at a time below the euphotic zone where light-based geolocation is impossible (Skomal et al., 2009). We have recently developed a new geolocation approach that combines all the physical data collected from archival tags, including light levels and depth-temperature profiles, in a likelihood framework to more accurately track the movements of tagged fishes in the ocean (Braun et al., 2018a, Chapter 2). Our method uses a purely diffusive animal movement model (e.g. Brownian motion) with behavior state switching (migratory or resident states based on a priori movement speeds) coupled with observations of the environment (e.g. in situ or modeled oceanography) to estimate the posterior distribution of the state (e.g. animal position and behavior) in a hidden Markov model (HMM) framework. Depth-temperature profiles provide diagnostic oceanographic signatures that, along with other data sources like light, SST, and maximum depth, may be leveraged to help constrain position (Aarestrup et al., 2009; Skomal et al., 2009).

Satellite tags have been deployed on basking sharks in the Atlantic since the pioneering work of Priede (1984). Yet, basking shark movements and ecology remain poorly understood. Here, we present the results of an intensive tagging effort that deployed 57 PSAT tags on adult basking sharks during summer months in waters adjacent to Cape Cod, Massachusetts. Profiles recorded by the tags were integrated with high-resolution oceanographic model outputs and in situ climatological data to construct likelihoods and improve geolocation estimates for basking sharks. The data provide a rare assessment of the large-scale movements and migratory behavior of the ocean's second largest fish. The information is, in turn, a prerequisite for any attempts to estimate abundance and population structure of basking sharks in the Atlantic Ocean.

\subsection{Methods}

\subsubsection{Study area and tagging}

We opportunistically deployed a variety of PSAT tags on basking sharks near Cape Cod, Massachusetts (USA) in the Northwest Atlantic (NWA) between 2004 and 2011 (Table 31). Total length of each individual was estimated relative to the tagging vessel and, where possible, the pelvic region was visually inspected to determine sex. Tags were applied by a professional harpoon fisherman into the dorsal musculature near the base of the first dorsal fin (Chaprales et al., 1998). This research was performed in accordance with the Woods Hole 
Oceanographic Institution's Animal Care and Use Committee (IACUC) protocol \#16518.

\subsubsection{Tag types}

Three types of PSAT tags were deployed on basking sharks (Table 3-1). These tags (Models Mk10-PAT, Mk10-AF, miniPAT; Wildlife Computers, Inc., WA, USA) logged depth, temperature, and light level data every 10 seconds (Mk10-AF) or 15 seconds (Mk10-PAT, miniPAT) to onboard memory. All tags recorded light data for geolocation purposes, and the Mk10-AF tag housed a Fastloc GPS receiver for acquiring high-resolution location information. Software in the tags summarized the high-resolution archived data into depthtemperature profiles at 8 depths (between minimum and maximum depth occupied for the summary period) for a 6,12 , or 24 -hour period depending on tag programming. These data were compiled into a single daily summary profile for data analysis. Tags also transmitted a summary of an individual's time of occupation within designated depth or temperature bins at 6,12 , or 24-hour resolution that was also compiled into daily summaries. Depth and temperature bin number, resolution, and extent differed slightly among tag type and year of tag deployment, but all were compiled to encompass the same depth $(<10,10-25$, 25-50, 50-200, 200-400, 400-1000, $>1000 \mathrm{~m})$ and temperature bins $(<7,7-9,9-11,11-13$, $\left.13-15,15-17,17-19,19-21,21-23,23-25,>25^{\circ} \mathrm{C}\right)$ for subsequent analysis. Results from the compilation of this time-at-depth and time-at-temperature data represented percent time of each deployment day that an individual occupied each of the common depth or temperature bins (shown above). Seasons were delimited in the analyses by the respective solstice and equinox dates for a given year.

At pre-programmed dates during tag deployment (range of programmed deployment duration 129-361 days), tags were released from the animal using a corrosive burn wire. After the tags released and floated to the surface, summarized data were transmitted to Argos satellites until battery failure. Transmitted data were decoded with manufacturer software (WC-DAP 3.0, Wildlife Computers, Inc., Redmond, WA), and light-based geolocation estimates were calculated and evaluated using tag manufacturer software (WC-GPE2). All subsequent analyses were conducted in the R Statistical Environment (R Development Core Team, 2015).

\subsubsection{Geolocation methods}

We estimated most probable tracks for PSAT-tagged basking sharks using the HMMoce package (Braun et al., 2018a, Chapter 2) for R (R Development Core Team, 2015). This approach leverages light-levels, SST, depth-temperature profiles, and maximum depth data recorded by PSAT tags, with empirical oceanographic data and model outputs, to construct likelihoods of the tagged individual's movements. Likelihoods are convolved in a spatially-gridded hidden Markov model that computes posterior probability distributions to estimate the most 
likely state (position and behavior) of the animal at each time point, which was typically daily. Parameter estimation is performed on a $1^{\circ}$ grid (for improved computation speed), and full model runs use a $0.25^{\circ}$ grid. In double-tagging experiments, HMMoce was shown to recreate movement trajectories with mean pointwise error of $141 \mathrm{~km}$ (range 93-183 km, $\mathrm{n}=4$ ) based on light and SST data that represented only $25 \%$ and $50 \%$ of the deployment days, respectively (Braun et al., 2018a, Chapter 2), although the geolocation error will likely vary with oceanographic regime and animal behavior.

Briefly, HMMoce estimates location and behavior from electronic archival tags. This involves: 1) calculating spatially-gridded observation likelihoods at each time point based on tag and environmental data; 2) forming the state-space model and estimating model parameters; and 3) model selection and interpretation. At each daily time step, we calculate a likelihood of the animal's position $L\left(x_{t}\right)$ on the grid:

$$
L\left(x_{t}\right)=L_{1}\left(x_{t}\right) \cdot L_{2}\left(x_{t}\right) \ldots L_{n}\left(x_{t}\right)
$$

where 1: $n$ indicates individual, observation-based likelihoods formed for each type of input data at each time point (e.g. $\left.L_{S S T}\left(x_{t}\right)\right)$.

Observation-based likelihoods were derived from in situ SST, light-based longitude, and depth-temperature profile data collected by the tags, using five separate likelihood calculations as follows. 1) An SST likelihood was generated for tag-based SST values integrated according to an error term $( \pm 1 \%)$ and compared to remotely-sensed SST from daily optimally-interpolated SST (OI-SST, $0.25^{\circ}$ resolution) fields (Reynolds et al., 2007; Banzon et al., 2016). 2) Light-based longitude likelihood was derived using estimates of longitude from GPE2 software (Wildlife Computers, Inc.), which facilitated visual checking of light curves. Depth-temperature profiles recorded by the tag were compared to 3) daily reanalysis model depth-temperature products from the HYbrid Coordinate Ocean Model (HYCOM, $0.08^{\circ}$ resolution; Bleck, 2002; Chassignet et al., 2007), and 4) monthly climatological mean depth-temperature data from the World Ocean Atlas 2013 (0.25 $5^{\circ}$ resolution; Locarnini et al., 2013) at standard depth levels available in these products. Individual likelihood surfaces for each depth level were then multiplied together for an overall profile likelihood at that time point. 5) Ocean Heat Content (OHC) was obtained by integrating the heat content of the water column above the minimum daily temperature to the most shallow depth recorded by the tag for both the tag profiles and HYCOM fields (Luo et al., 2015).

All observation-based likelihoods were formed using integrated likelihood calculations (Le Bris et al., 2013). For example, daily SST likelihoods were constructed as:

$$
L_{S S T}\left(x_{t}\right)=\int_{S S T_{\min }}^{S S T_{\max }} N\left(t ; \mu_{z}, \sigma_{z}\right) d z
$$

where $N$ is a normal probability distribution function, $\mu_{z}$ the remotely-sensed SST grid 
cell value, and $\sigma_{z}$ the grid cell standard deviation. The same integration approach was performed on the other observation likelihoods. For 3D likelihoods, this approach was performed at each relevant standard depth level in the environmental dataset and integrated limits were tag-based minimum and maximum temperatures recorded (or predicted by linear regression) at that depth level. Standard deviation for all likelihood calculations was calculated with a "moving window" mean using the focal function in the raster package (Hijmans, 2016) for $\mathrm{R}$ to incorporate approximately $0.25^{\circ}$ of environmental data around each grid cell. Start and end locations and available GPS data (from the MK10-AF tag) were seeded as known positions in all model runs.

The resulting observation likelihoods (in various combinations; Table 3-1) were used in a two-step Bayesian state-space approach to estimate the posterior distribution of the state (in this case, a joint probability distribution of location and behavior at each time point). We considered "resident" and "migratory" behavior states that corresponded to fixed speeds of $0.4 \mathrm{~m} \cdot \mathrm{s}^{-1}\left(34.5 \mathrm{~km} \cdot \mathrm{d}^{-1}\right)$ for residency (following Curtis et al., 2014) and an order of magnitude higher $\left(4 \mathrm{~m} \cdot \mathrm{s}^{-1}, 345 \mathrm{~km} \cdot \mathrm{d}^{-1}\right)$ for migratory movements. These speeds represent maximum diffusion allowed per day $\left(1,200\right.$ and $120,000 \mathrm{~km}^{2} \cdot d^{-1}$ for resident and migratory daily diffusion, respectively) and were represented by Gaussian kernels (see documentation for HMMoce: gausskern for more information) that were convolved with observation likelihoods at each time point. Probability distributions were first calculated forward in time using alternating time and data updates of the current state estimate using a HMM filter on a $0.25^{\circ}$ likelihood grid. Parameter estimation was performed using an iterative Expectation-Maximization framework (Woillez et al., 2016). The HMM smoother recursion was the final step that worked backwards in time using filtered state estimates and all available observation data to determine smoothed state estimates. This step provided the time marginal of the probability distributions based on observations (posterior distributions). Distributions are summed for each behavior state and time step to determine the most likely behavior state for each time step. HMMoce calculates the mean or mode of the posterior distribution grid, at each time step, to estimate the animal's most probable track. Model selection was performed using Akaike Information Criterion (AIC). Resulting most probable track estimates represented daily location and most likely behavior state at that time point. Cumulative track distances were calculated using great-circle distance calculations between estimated daily locations using the rdist.earth function in the fields (Nychka et al., 2015) package for $\mathrm{R}$.

The posteriors were summed across behavior states for additional inference on seasonal habitat use, conceptually similar to a residency (see Eq. 5, Pedersen et al., 2011b) or utilization distribution (Royle and Dorazio, 2008). This approach was used to incorporate uncertainty around most probable track estimates that is included in the posteriors, as opposed to traditional utilization distribution calculations such as kernel density (e.g. Berumen 

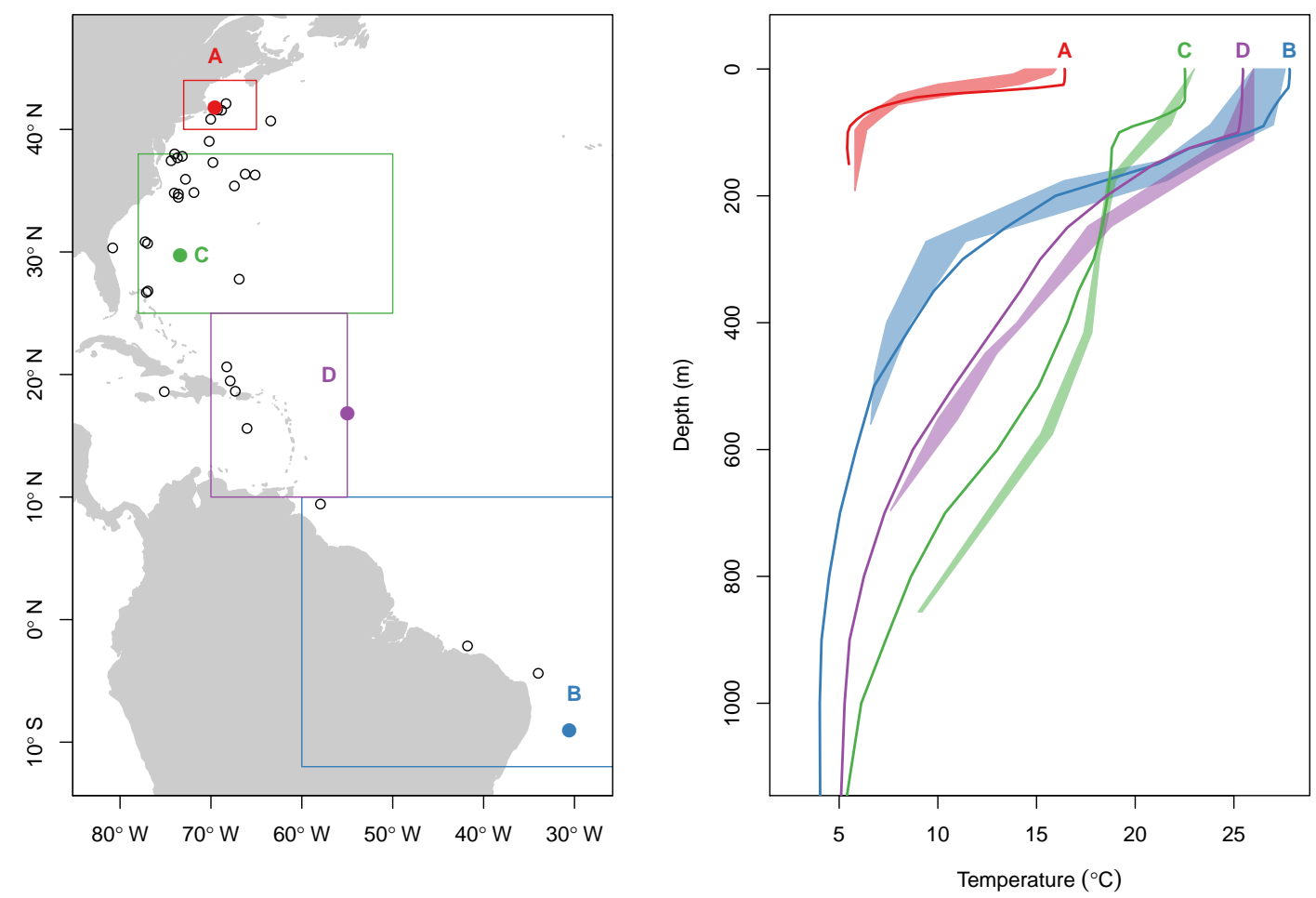

Figure 3-1: Example depth-temperature profile data from known pop-up locations of PSAT-tagged basking sharks. Selected, representative pop-up locations (color, left panel) from distinct regions of the study area were used to compare tag-based depth-temperature profiles (shaded from minimum to maximum recorded profile temperatures, right panel) to HYCOM profiles (lines, right panel) from the same time and location. Black circles (left panel) represent all tag pop-up locations in this study. Bounding boxes show the oceanographic regions discussed in the text, Figure 3-7 and Table 3-2.

et al., 2014).

\subsection{Results}

We tagged 57 basking sharks spanning sub-adult $(\sim 500-600 \mathrm{~cm})$ and adult $(>600-700$ $\mathrm{cm}$ ) life stages (range 549-762 cm males, 549-823 cm females) and both sexes (10 females, 3 males, 31 unknown). Forty-five (79\%) of the 57 PSAT tags deployed between 2004-2011 reported. Eight tags released prematurely, and 1 of the tags had no useable data. Data from 37 of the remaining 44 tags contained sufficient information for further analysis (Table 31). These deployments averaged 234 days (SD 85 days, range 79-424 days). There was no evidence of tagging-induced mortality. Of the 35 tags that transmitted data (excluding two that were physically recovered), we received data representing $7 \%$ (median, range 1$44 \%$ ), $26 \%$ (median, range 4-61\%), and 52\% (median, range $7-91 \%$ ) of deployment days with light-based position estimates, SST, and depth-temperature profile data, respectively. 


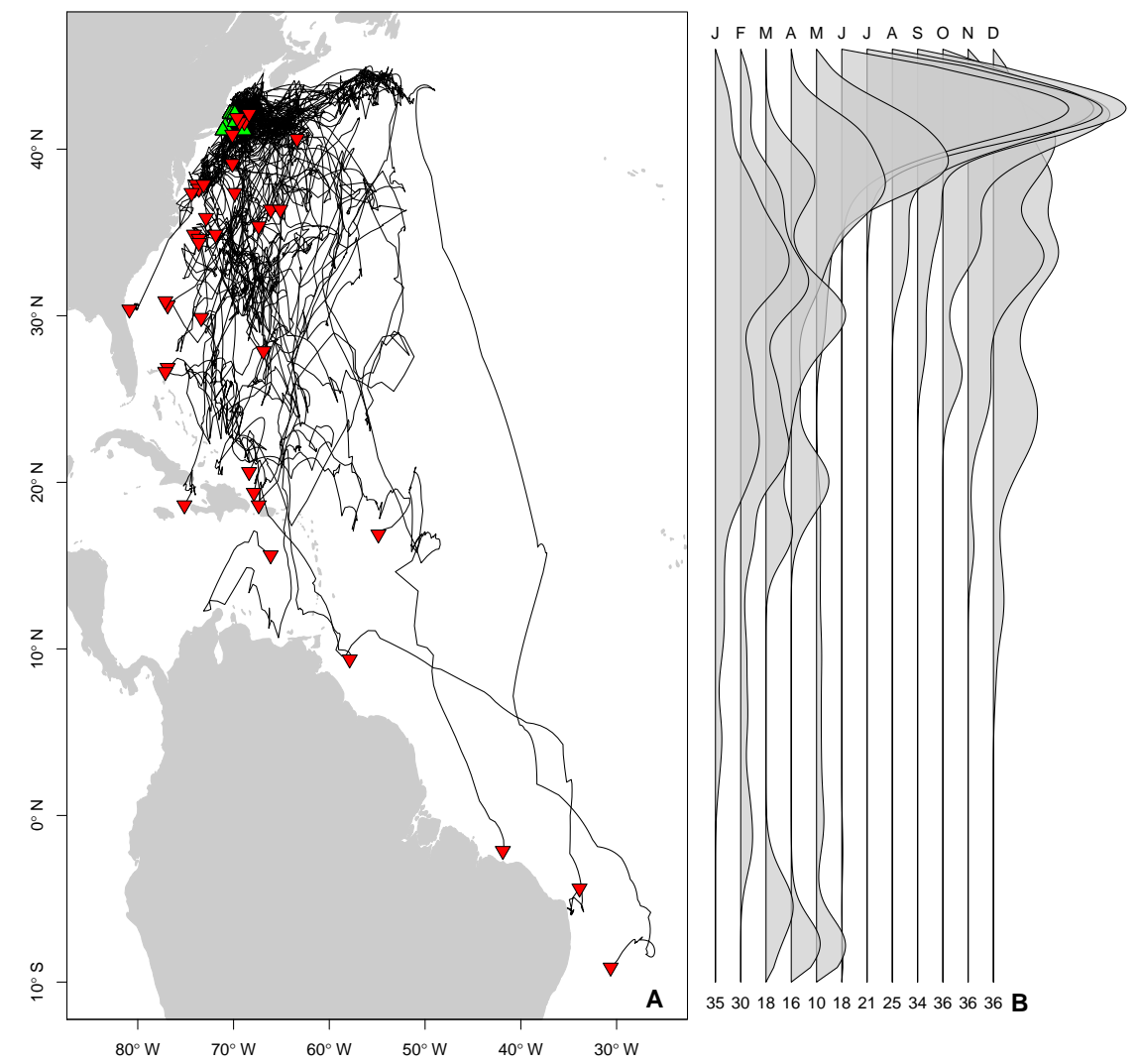

Figure 3-2: Most probable tracks (panel A) and latitude density by month (panel B) for 37 basking sharks satellite tagged off New England during June through October of 2004-2011. Tracks are plotted as black lines, and green and red triangles represent tag and pop-up locations, respectively. Letters above the density plot indicate month (e.g. $\mathrm{F}=$ February), and numbers below indicate the number of individuals with tag data during that month.

The remaining two tags were physically recovered: one tag washed ashore in the Bahamas after 133 days at liberty and one was located on a beach in Rhode Island still attached to the deceased shark after a 78 day deployment. The full archival record was analyzed for these two deployments and contained light-based position estimates and SST data for 5-51\% and $66-89 \%$ of deployment days, respectively, during which the animal occupied the surface (SST) or euphotic zone (light). Transmitted and archival profile data was available for more deployment days than either light-based position estimates or SST data in all but one of the reporting tags. One individual (B28) was tagged with a Fastloc GPS tag which reported 4 GPS snapshots over 3 days during winter (Dec. 22, 23, 26). These locations were fixed in the model runs for this individual, and no other usable GPS positions were acquired.

For a given tag, varying amounts of each data type were obtained due to behavioral variability and individual differences in data transmission. Model selection favored HYCOM- 
based profile likelihoods (Figure 3-1) in 34 of 37 track calculations. Of the remaining 3 individual geolocation analyses, one favored OHC-based profile likelihoods, one WOA-based profile likelihoods and one model selection used only light and SST observations. Available light and SST data were not used in the selected model for 4 and 6 individual tag datasets, respectively (Table 3-1). Nearly all model outputs indicated the "migratory" behavior state was more likely once the tagged individual left the New England shelf $(76 \%$ of off-shelf position estimates), and this behavior remained dominant throughout the Sargasso Sea region ( $77 \%$ of off-shelf position estimates). Shelf habitats near New England and from the Antillean Arc to the Amazon Delta were characterized by a higher likelihood ( $~ 50 \%$ of on-shelf position estimates) of "resident" behavior (e.g. slower, more tortuous movements).

While all tags were deployed off the northeastern coast of the U.S., most probable tracks showed a wide range of individual movements (Figure 3-2). For individuals with sufficient data to perform the geolocation analysis $(n=34)$, track distances ranged from 4,009-17,387 $\mathrm{km}$ (mean 10,136 \pm 3,988 SD) spanning 79-424 days (mean $207 \pm 107 \mathrm{SD}$ ). Several of the sharks showed relatively directed, long-range movements south from the tagging location in New England to the Puerto Rico Trench $(\mathrm{n}=4)$, Antillean Arc $(\mathrm{n}=3)$, and Amazon Delta $(\mathrm{n}=3)$ up to $17,387 \mathrm{~km}$ (6,200 $\mathrm{km}$ displacement) from the tagging location (Figure 3-5). Three individuals made transequatorial movements.

Movements of tracked sharks demonstrated strong seasonality (Figure 3-2, Figure 33) with individuals occupying coastal waters in high latitudes during the summer before moving south in fall (Figure 3-2, Figure 3-3), and all but one individual (B26) departed New England by January. This individual remained along the shelf edge between New England and the Grand Banks for the winter and returned to the New England canyons by late February (B26 in Figure 3-4). All other tagged sharks overwintered in habitats as close as the Sargasso Sea and as far as the northeastern coast of Brazil before beginning to return to New England waters in late spring and early summer (Figure 3-2, Figure 33 ). Seven tags were deployed for $>300$ days, including one for 423 days, and five of them transmitted sufficient data for track estimation. Six of these seven tags popped up in New England waters approximately one year after tagging, while the remaining tag reported near the Amazon Delta and represented the furthest southerly movements observed in this study (Figure 3-2, Figure 3-5). Eighteen tags exhibited deployment durations $>250$ days, 10 of which $(59 \%)$ exhibited return migrations to the NWA, including one pop-up location $60 \mathrm{~km}$ from the tagging location one year later (B21). There was no significant difference in mean track distance between males and females (t-test, $p=0.4633$ ), although male sample size was low $(\mathrm{n}=3)$, and a linear regression analysis found no significant relation between shark size and extent of movement $\left(p=0.27, R^{2}=0.05\right)$ or minimum latitude occupied $(p=0.48$, $\left.R^{2}=0.02\right)$. 
Table 3-1: Summary information from satellite tag deployments on Cetorhinus maximus in the NW Atlantic.

\begin{tabular}{|c|c|c|c|c|c|c|c|c|c|c|c|c|c|}
\hline $\begin{array}{l}\text { Shark } \\
\text { ID }\end{array}$ & $\begin{array}{l}\text { Tag } \\
\text { Type }\end{array}$ & Tag Date & $\begin{array}{l}\mathrm{TL} \\
(\mathrm{m})\end{array}$ & Sex & $\begin{array}{l}\text { Pop } \\
\text { Lat } \\
\left({ }^{\circ} \mathbf{N}\right)\end{array}$ & $\begin{array}{l}\text { Pop } \\
\text { Lon } \\
\left({ }^{\circ} \mathbf{W}\right)\end{array}$ & $\begin{array}{l}\text { TAL } \\
\text { (d) }\end{array}$ & $\begin{array}{l}\text { Depth } \\
(\mathrm{m})\end{array}$ & $\begin{array}{l}\text { Dist. } \\
(\mathrm{km})\end{array}$ & $\begin{array}{l}\text { Light } \\
(\%)\end{array}$ & $\begin{array}{l}\text { SST } \\
(\%)\end{array}$ & $\begin{array}{l}\text { PDT } \\
(\%)\end{array}$ & Obs. \\
\hline B01 & MK10 & 2004-09-24 & 7.6 & & 30.33 & 80.81 & 129 & 84 & 4,010 & 22 & 25 & 41 & LW \\
\hline $\mathrm{B}^{2} 2^{a}$ & MK10 & 2004-09-24 & 9.8 & & 18.60 & 75.13 & 129 & $980^{d}$ & 4,769 & 12 & 27 & 74 & $\mathrm{LH}$ \\
\hline B03 & MK10 & $2005-07-21$ & 6.3 & & 34.81 & 74.03 & 254 & 940 & 10,568 & 1 & 10 & 43 & LSH \\
\hline $\mathrm{B} 04^{b}$ & MK10 & $2005-07-21$ & & & 27.78 & 66.89 & 194 & $980^{d}$ & 9,756 & 13 & 26 & 47 & LSH \\
\hline $\mathrm{B} 05^{a, b}$ & MK10 & 2005-07-21 & 7.3 & & -4.38 & 33.99 & 254 & $980^{d}$ & 13,449 & 8 & 16 & 38 & LSH \\
\hline $\mathrm{B}^{2} 6^{a}$ & MK10 & $2005-08-26$ & 7.7 & & 9.43 & 57.95 & 173 & $980^{d}$ & 8,566 & 6 & 7 & 48 & LSH \\
\hline $\mathrm{B} 07^{b}$ & MK10 & $2005-08-26$ & 7.0 & & 37.30 & 69.78 & 173 & $980^{d}$ & 8,172 & 17 & 29 & 63 & LSH \\
\hline $\mathrm{B} 08^{b}$ & MK10 & $2005-07-21$ & 7.1 & & 19.48 & 67.88 & 209 & 892 & 11,390 & 12 & 19 & 52 & LSH \\
\hline B09 & MK10 & $2005-03-10$ & 8.0 & & -2.15 & 41.77 & 241 & $980^{d}$ & 11,446 & 3 & 10 & 41 & $\mathrm{LH}$ \\
\hline $\mathrm{B} 10^{a}$ & MK10 & $2005-07-21$ & 6.4 & & 26.81 & 76.93 & 209 & $980^{d}$ & 11,583 & 3 & 15 & 56 & LSH \\
\hline B11 & MK10 & 2005-03-10 & 7.7 & & 36.35 & 66.23 & 241 & $980^{d}$ & 11,079 & 2 & 14 & 44 & LSH \\
\hline $\mathrm{B} 12^{b}$ & MK10 & 2005-07-21 & 7.7 & & 26.70 & 77.11 & 194 & $980^{d}$ & 5,380 & 16 & 33 & 56 & $\mathrm{LH}$ \\
\hline $\mathrm{B} 13^{b, c}$ & MK10 & $2005-06-18$ & 7.2 & $\mathrm{~F}$ & 38.00 & 74.00 & 78 & $980^{d}$ & 4,641 & 51 & 89 & 100 & $\mathrm{LS}$ \\
\hline $\mathrm{B} 14^{b}$ & MK10 & 2005-03-07 & 8.1 & & 42.25 & 70.66 & 423 & 900 & $96^{e}$ & 8 & 11 & 11 & DD \\
\hline B15 & MK10 & $2005-03-07$ & 5.9 & & 30.69 & 76.97 & 196 & $980^{d}$ & 8,642 & 2 & 17 & 49 & $\mathrm{SH}$ \\
\hline B16 & MK10 & 2006-05-09 & 8.2 & $\mathrm{~F}$ & 40.99 & 69.46 & 8 & 152 & 9,969 & 36 & 51 & 61 & LSH \\
\hline $\mathrm{B} 17^{a}$ & MK10 & 2006-06-09 & & & 37.68 & 73.68 & 268 & 600 & $567^{e}$ & & & & $\mathrm{DD}$ \\
\hline $\mathrm{B} 18^{a}$ & MK10 & 2006-06-09 & & & 37.45 & 74.38 & 268 & 1040 & 11,157 & 7 & 14 & 54 & $\mathrm{LH}$ \\
\hline B19 & MK10 & $2008-11-10$ & & & 41.56 & 68.83 & 355 & 1232 & 16,499 & 13 & 26 & 45 & LSH \\
\hline B20 & MK10 & 2008-11-10 & & & 41.60 & 69.28 & 294 & 1328 & 13,548 & 4 & 24 & 59 & LH \\
\hline B21 & MK10 & 2008-11-10 & & & 41.82 & 69.55 & 355 & 1088 & 14,684 & 5 & 20 & 48 & LSH \\
\hline B22 & MK10 & $2008-11-10$ & & & 40.83 & 70.03 & 355 & 1040 & 15,931 & 11 & 23 & 46 & LSH \\
\hline $\mathrm{B} 23$ & MK10 & $2008-11-10$ & & & 42.11 & 68.34 & 355 & 1144 & 15,107 & 6 & 18 & 36 & LSH \\
\hline B24 & MK10 & 2008-11-10 & & & 42.08 & 70.33 & 5 & 80 & $4^{e}$ & & & & $\mathrm{DD}$ \\
\hline B25 & MK10 & $2008-11-10$ & & & 40.89 & 70.26 & 16 & 136 & $134^{e}$ & & & & $\mathrm{DD}$ \\
\hline B26 & $\mathrm{mP}$ & $2010-08-21$ & & & 40.69 & 63.42 & 189 & 688 & 7,051 & 14 & 56 & 91 & LS \\
\hline $\mathrm{B} 27$ & $\mathrm{mP}$ & 2011-05-06 & 6.7 & $\mathrm{~F}$ & 42.04 & 69.14 & 12 & 300 & $65^{e}$ & & & & $\mathrm{DD}$ \\
\hline B28 & $\mathrm{mP}$ & 2011-05-06 & 7.6 & $\mathrm{~F}$ & 42.44 & 68.69 & 8 & 232 & $107^{e}$ & & & & $\mathrm{DD}$ \\
\hline B29 & $\mathrm{mP}$ & 2011-08-06 & 7.6 & & 30.83 & 77.24 & 298 & 1208 & 16,767 & 4 & 37 & 67 & LSH \\
\hline B30 & $\mathrm{mP}$ & 2011-08-06 & 6.1 & M & 39.03 & 70.19 & 298 & 1112 & 17,387 & 27 & 49 & 71 & LSH \\
\hline B31 & $\mathrm{mP}$ & 2011-05-06 & 5.5 & $\mathrm{~F}$ & 34.72 & 73.58 & 271 & 1112 & 10,235 & 40 & 60 & 73 & LSH \\
\hline B32 & $\mathrm{mP}$ & 2011-08-06 & 6.1 & $\mathrm{~F}$ & 34.46 & 73.59 & 268 & 1112 & 15,408 & 44 & 32 & 59 & LSH \\
\hline B33 & $\mathrm{mP}$ & 2011-08-06 & 5.5 & M & 37.81 & 73.14 & 299 & 1088 & 16,245 & 41 & 47 & 70 & LSH \\
\hline B34 & MK10 & $2011-06-27$ & 8.2 & $\mathrm{~F}$ & 42.27 & 69.23 & 340 & 1000 & $50^{e}$ & 1 & 4 & 7 & $\mathrm{DD}$ \\
\hline B35 & MK10 & 2011-06-27 & 7.6 & $\mathrm{~F}$ & 36.28 & 65.14 & 230 & 1112 & 6,794 & 5 & 13 & 38 & LSH \\
\hline B36 & MK10 & 2011-06-27 & 6.1 & $\mathrm{~F}$ & -9.02 & 30.57 & 340 & 1000 & 10,525 & 2 & 5 & 11 & LSO \\
\hline B37 & $\mathrm{mP}$ & 2011-06-27 & 7.6 & $\mathrm{~F}$ & 20.63 & 68.26 & 279 & 1020 & 10,739 & 4 & 34 & 68 & LSH \\
\hline B38 & MK10AF & $2011-08-23$ & 5.2 & & 29.76 & 73.38 & 121 & 1040 & 5,653 & 2 & 61 & 82 & SHF \\
\hline $\mathrm{B} 39^{c}$ & $\mathrm{mP}$ & 2011-09-21 & 5.5 & & 16.80 & 54.98 & 133 & 1208 & 7,192 & 5 & 66 & 100 & LSH \\
\hline $\mathrm{B} 40$ & MK10 & 2011-09-21 & 8.2 & & 34.85 & 71.87 & 133 & 936 & 5,454 & 22 & 49 & 52 & LSH \\
\hline B41 & MK10 & 2011-09-21 & 7.6 & M & 18.63 & 67.32 & 133 & 1020 & 5,757 & 1 & 13 & 39 & $\mathrm{SH}$ \\
\hline B42 & MK10 & 2011-09-21 & 6.1 & & 35.39 & 67.42 & 133 & 1504 & 5,495 & 5 & 46 & 54 & $\mathrm{SH}$ \\
\hline B43 & MK10 & 2011-09-21 & 6.7 & & 15.60 & 66.03 & 133 & 1272 & 7,675 & 4 & 27 & 43 & LSH \\
\hline B44 & MK10 & 2011-09-21 & 4.6 & & 35.93 & 77.80 & 133 & 1112 & 6,300 & 20 & 33 & 48 & LSH \\
\hline
\end{tabular}

Identification number of each individual is shown along with the tag model. All tags were manufactured by Wildlife Computers, Inc. (Redmond, WA, USA). TL $=$ the total length $(\mathrm{m})$ of the individual tagged as estimated from the tagging vessel; Sex = male (M) or female $(\mathrm{F})$ where determination was possible by visual observation of presence or absence of claspers between the pelvic fins, no entry indicates that sex could not be confidently determined; Pop Lat / Long $=$ coordinates of tag detachment location; TAL = number of days between tag deployment and detachment; Depth $=$ the deepest depth $(\mathrm{m})$ reported by the tag during the deployment; Dist $=$ cumulative distance of most probable track; Light, SST and depth-temperature profile (PDT) columns indicate percent of deployment days with light-based location estimates, sea surface temperature data and depth-temperature profiles. Obs. = observation likelihoods are those observations used in HMMoce to construct the most probable track for each tagged animal: $\mathrm{L}=$ lightbased longitude, $\mathrm{S}=$ sea surface temperature, $\mathrm{H}=\mathrm{HYCOM}$ depth-temperature profiles, $\mathrm{W}=$ World Ocean Atlas depthtemperature profiles, $\mathrm{O}=$ integrated Ocean Heat Content, $\mathrm{F}=$ Fastloc GPS, $\mathrm{DD}=$ data deficient. ${ }^{a}$ Tracks published in Skomal et al. (2004). ${ }^{b}$ Depth data published in Curtis et al. (2014). ${ }^{c}$ Tag was physically recovered. ${ }^{d}$ Maximum depth capability of this tag model. ${ }^{e}$ No track was constructed. This is a straight-line (displacement) distance from tagging location to pop-up. 
Long-distance migrations often co-occurred with large vertical excursions and led to occupation of several distinct water masses throughout the year. Binned vertical histogram data (Figure 3-3) were used to quantify where in the water column sharks tended to frequent. Overall, extensive vertical excursions characterized basking shark dive behavior when an individual left the continental shelf region of the eastern US (Figs. 3-3, 3-4, 3-6). Twentyone individuals spent time below $1000 \mathrm{~m}$, and it was likely that only limitations in earlier tag technology (maximum depth capability of $980 \mathrm{~m}$ ) prevented those individuals' tags from recording similar behavior. The maximum depth recorded by a tag (shark B42) was 1504 $\mathrm{m}$ and recorded temperatures at depth in this study ranged from $4.2-29.9^{\circ} \mathrm{C}$. Recorded SST values from all individuals ranged from $7.4-29.9^{\circ} \mathrm{C}$ (median $18.3^{\circ} \mathrm{C}$ ). Overall, $63 \%$ of basking shark depth-temperature data was $8-18^{\circ} \mathrm{C}, 87 \%$ was between $6-20^{\circ} \mathrm{C}$, and all individuals made occasional forays into temperatures well outside those bounds (Figure 3-7). In fact, one individual (B26) remained at northern latitudes (from Cape Cod to the Grand Banks) during winter and experienced $<10^{\circ} \mathrm{C}$ ambient water temperatures for $>3$ months (B26 in Figure 3-4; range $4.8-12^{\circ} \mathrm{C}$ from Nov 1 to Feb 15).

Vertical habitat envelopes described the distinct water masses across the study area (from coastal New England to open ocean off Brazil), their depth-temperature characteristics, and the vertical behavior observed in each water mass (Figure 3-7, Table 3-2). Generally, individuals spent much of their time in the epipelagic zone $(<200 \mathrm{~m})$ during summer months at northern temperate latitudes where temperatures were typically $<20^{\circ} \mathrm{C}$ (Figs. 3-4, 36, 3-7). However, during the fall, the majority of tagged individuals transitioned from the epipelagic orientation of the summer months to residency in the mesopelagic zone during the winter in which they cumulatively spent $>60 \%$ of time between 400-1000 m (Figs. 3-3, 3-4, 3-6). Based on depth-temperature profile data, sharks remained below the euphotic zone for $27 \%$ (median; range 0-90\%) of fall, winter and spring deployment days for which data existed, and this behavior exhibited no relationship with individual size or sex, although male sample size was low $(n=3)$. Temperature profiles from these periods of mesopelagic occupation indicated this behavior occurred largely in the Sargasso Sea where warm (14$20^{\circ} \mathrm{C}$ ) water penetrates deep in the water column (profile $\mathrm{C}$ in Figures 3-1 and 3-7) resulting in relatively warm water at depth (e.g. B20 and B22 in Figures 3-6 and 3-7). However, some sharks overwintered further south in the Guyana Basin and off the Brazilian shelf as indicated by warmer surface temperatures and a stronger temperature gradient with depth (e.g. profile B in Figure 3-1, B36 in Figures 3-6 and 3-7). Sharks generally inhabited warmer waters throughout winter at low latitudes, despite prolonged deep-water occupation, than the surface waters that they inhabited during summer months (Figure 3-7, Table 3-2).

Shark B22 provided a good example of the distinct water masses traversed during a one-year deployment, with a complete round trip migration starting and ending in the tagging region (Figs. 3-4, 3-5). This individual occupied a well-mixed, cool surface layer 

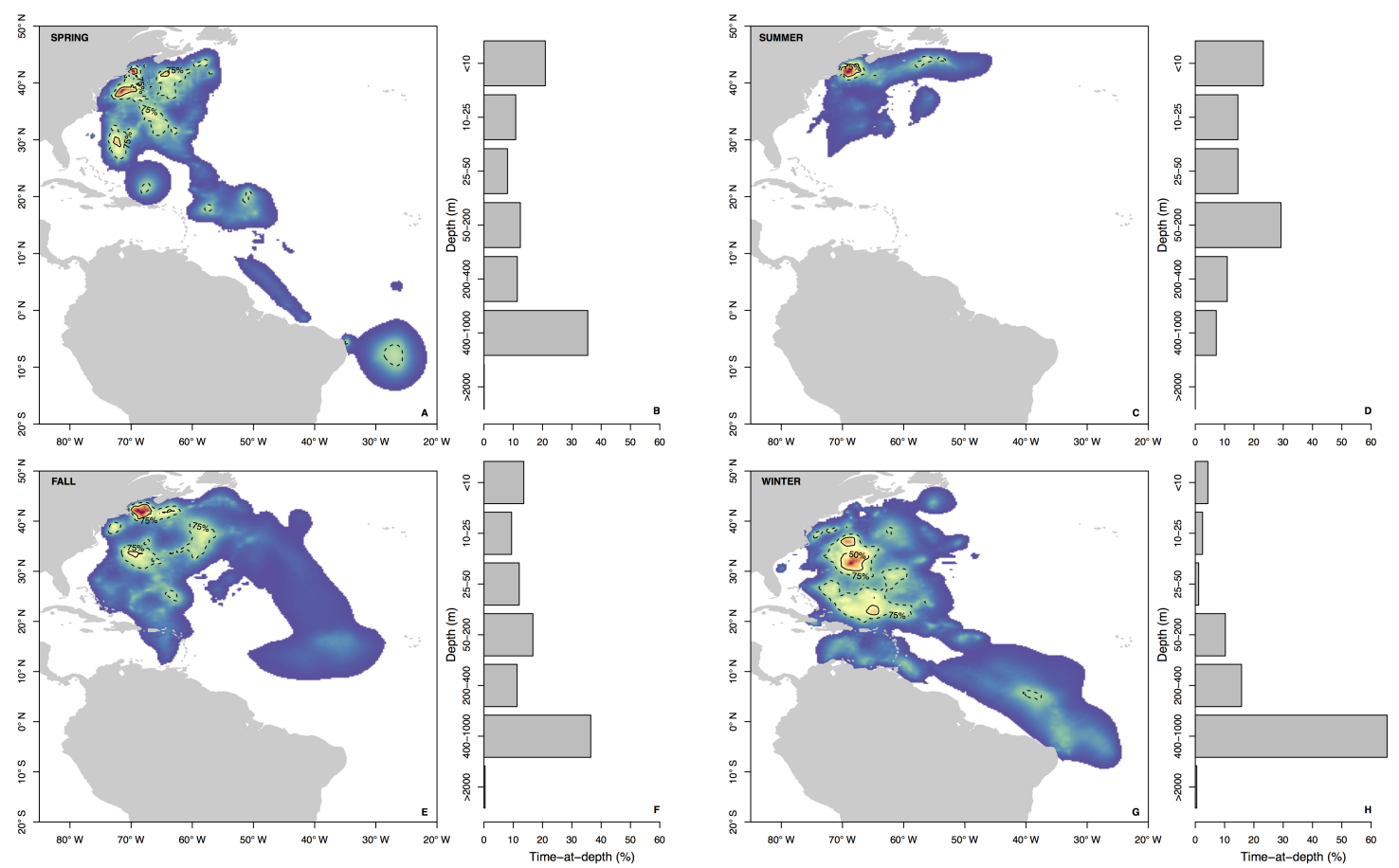

Figure 3-3: Seasonal residency distributions (panels A, C, E, G) and cumulative time-at-depth (panels B, D, F, H) for spring (A, B), summer (C, D), fall (E, F), and winter (G, H). Residency distributions were calculated using the HMMoce package for R. Contour lines represent $50 \%$ and $75 \%$ of occupation for a given season as depicted by solid and dashed contours, respectively.

in the Gulf of Maine during October before moving through the Gulf Stream and into the northern Sargasso Sea in November. This individual occupied the northern Sargasso from December to March before moving back into a more uniformly cool layer in April and May near Cape Hatteras. By June, both the estimated track and water characteristics indicate this individual had returned to the shelf-edge waters near New England and onto the shelf near Cape Cod by late September (Figure 3-4).

\subsection{Discussion}

It is increasingly clear that pelagic fishes throughout the global ocean conduct long-range migratory movements (e.g. Block et al., 2011; Skomal et al., 2017) and connect the surface and deep ocean through meso- and bathypelagic dive behavior (Braun et al., 2014; Thorrold et al., 2014). The basking sharks tagged in the present study were no exception, making some of the longest horizontal movements of any ocean species tagged to date (Block et al., 2005; Bonfil, 2005; Hays et al., 2006; Skomal et al., 2017). Tagged individuals moved through several distinct water masses of the western Atlantic, and spent significant time in the mesopelagic, demonstrating the ability of basking sharks to traverse a wide range of environments from the surface to deep ocean across a $25^{\circ} \mathrm{C}$ temperature range. 

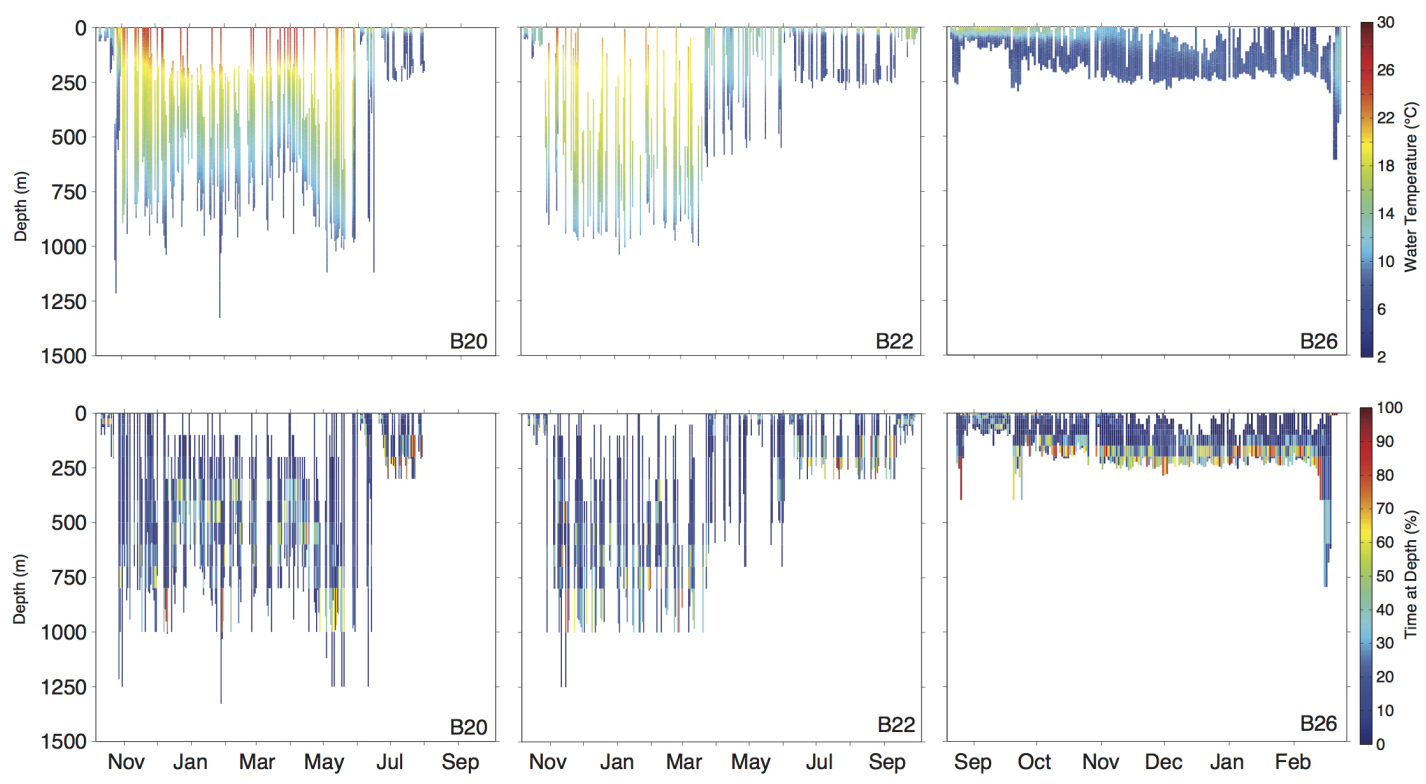

Figure 3-4: Daily depth-temperature profiles (row 1) and time-at-depth profiles (row 2) for 3 representative basking sharks (tracks plotted in Figure 3-5A). Note differing time scale (x-axis) among individuals.

Movements through distinct water masses often coincided with varying periods of deep water occupation. Nearly all tagged individuals demonstrated a shift to residency from surface waters to deep in the meso- and bathypelagic during colder months that may explain the apparent disappearance of basking sharks during winter (Parker and Boeseman, 1954). While our results corroborate previous studies that suggest seasonally variable dive behavior (Sims et al., 2003) and southward migration during winter (Doherty et al., 2017), sharks in this study made much more extensive movements throughout the open ocean than those observed in similar studies elsewhere (Doherty et al., 2017) and spent up to several months at mesopelagic depths. Sharks tagged in the northeast Atlantic (NEA) did make dives to similar maximum depths ( $50 \%$ of tagged individuals dove below $1000 \mathrm{~m}$; Doherty et al. 2017 ) but averaged $>80 \%$ of time above $200 \mathrm{~m}$ and $<10 \%$ deeper than $500 \mathrm{~m}$ (Sims et al., 2003; Doherty et al., 2017). The mesopelagic occupation observed in this study suggests this behavior is much more ubiquitous among NWA basking sharks as they move throughout the open ocean than their NEA conspecifics that are relatively close to the coast and may be a product of the oceanography experienced (e.g. warm, homogenous depth-temperature profiles in the Sargasso Sea) by these individuals in the open ocean of the NWA.

The other main difference in behavior among these regions is the winter migration strategy. NEA basking sharks moved south from Ireland and the UK to the Bay of Biscay, but despite tagging 70 basking sharks with satellite tags, only 1 individual traversed $>20^{\circ}$ of latitude after summer occupation of the far northern latitudes (Doherty et al., 2017). In 

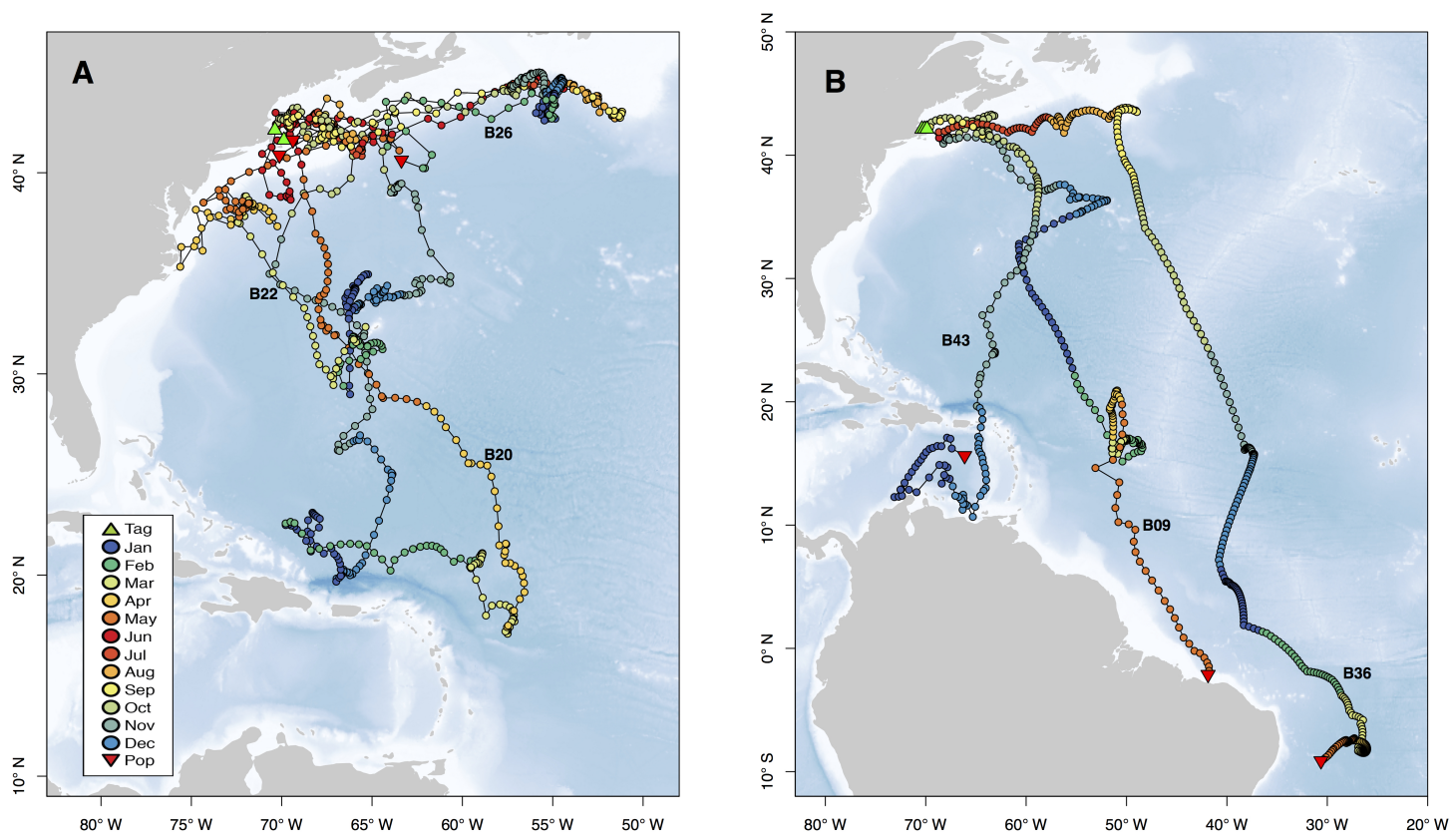

Figure 3-5: Movements of selected individuals demonstrating representative behaviors exhibited by sharks in this study. Two selected individuals exhibited site fidelity to Cape Cod (B20, B22) and one individual overwintered near Newfoundland (B26, panel A). The variety of long distance movements are represented by three individuals with pop-up locations from the eastern Caribbean to the SE coast of Brazil (panel B). Tracks are plotted as points colored by month, and green and red triangles represent tag and pop-up locations, respectively. Text labels correspond to Shark ID in Table 3-1, and blue background indicates bathymetry of the region. Vertical habitat use of these selected individuals is shown in Figure 3-4 and Figure 3-6.

contrast, winter movements at and beyond this scale were more commonly observed in the NWA (Skomal et al., 2009, and this study). These movements refute the suggestion that basking sharks are largely restricted to temperate latitudes (Sims, 1999; Sims et al., 2003; Gore et al., 2008; Doherty et al., 2017), while NEA basking sharks did not traverse the equator, movements by individuals in the NWA demonstrate that tropical environments do not pose a barrier to basking shark movements.

It seems likely that the long-distance movements by basking sharks that we documented are driven, at least in part, by the dynamic oceanic environment of the western Atlantic Ocean. The NWA is punctuated by strong seasonal fluxes in pelagic primary productivity (Miller and Wheeler, 2012) and temperature (Talley, 2011). The warm water and high productivity attract many species to the temperate NWA during summer (e.g. basking sharks, Curtis et al. 2014; white sharks, Skomal et al. 2017). While it is clear basking sharks are able to tolerate sub- $10^{\circ} \mathrm{C}$ water for months at a time (B26 in Figure 3-4; Sims 2008), individuals in this study spent much of their time overwintering in warm, mesopelagic waters. In fact, as a whole, sharks spent more time in warmer water during deep occupation periods 

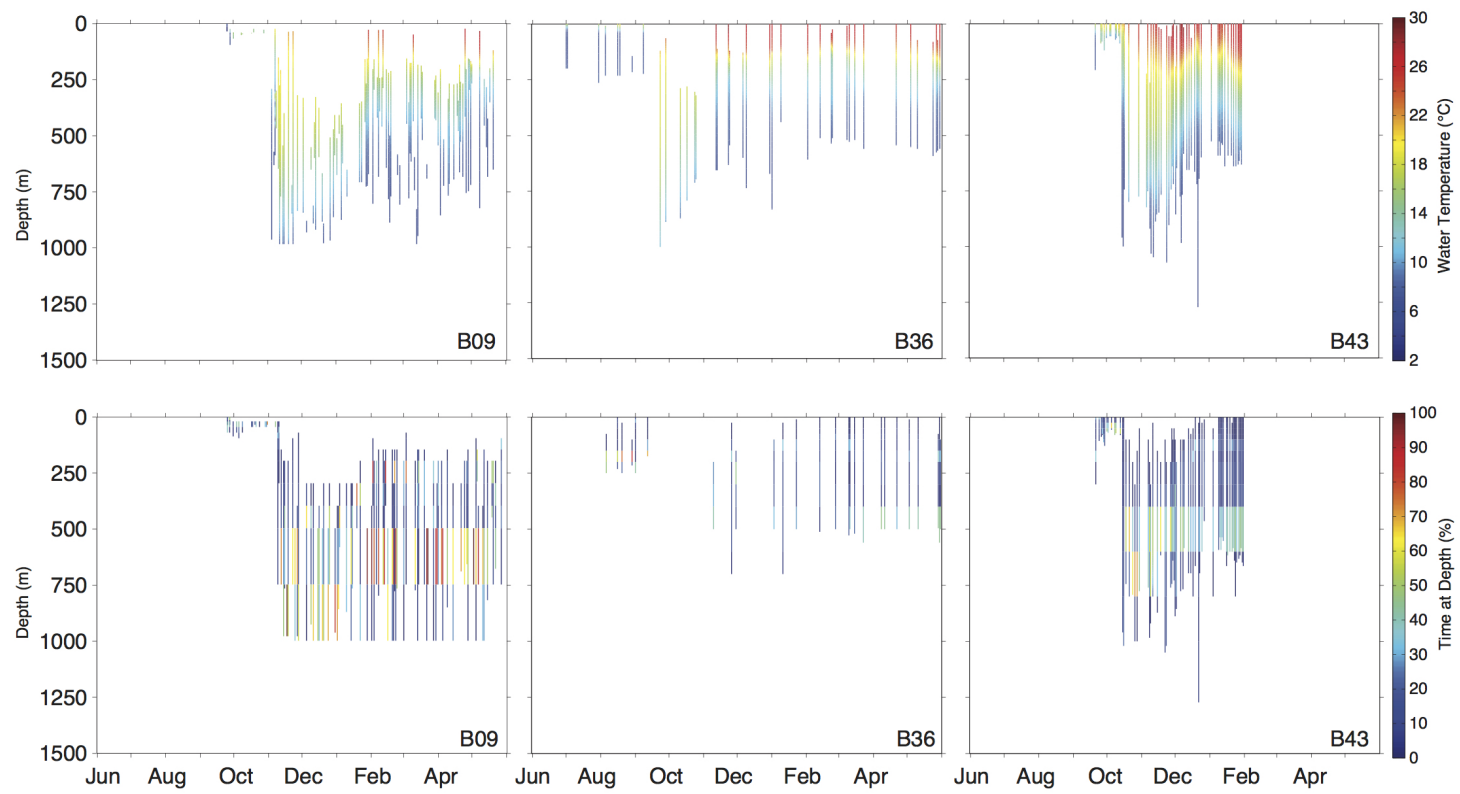

Figure 3-6: Daily depth-temperature profiles (row 1) and time-at-depth profiles (row 2) for 3 representative basking sharks (tracks plotted in Figure 3-5B). Note differing time scales (x-axis) among individuals.

in winter as they moved south than they did during summer. While the function of this deep occupation is unknown, the Sargasso Sea is a relatively stable, warm water mass during winter months and may host prey opportunities for basking sharks in the mesopelagic, including a substantial deep scattering layer that overlaps with basking shark depth use (400-600 m; Irigoien et al. 2014) and potentially co-occurring anguillid eel spawning aggregations (Wysujack et al., 2015). These migrations away from the northern winter may also be associated with hotspots of relatively high production at lower latitudes (e.g. Brazilian shelf; Mourato et al., 2014). Movements in this study demonstrated orientation to shelf edge habitats, particularly along the northern coast of Brazil during winter, that likely host persistent fronts (Le Fèvre, 1987; Sims, 2008) and thus relatively high primary production even at low latitude. While basking sharks have been shown to orient to persistent seasonal fronts (Miller et al., 2015), most individual tracks in this study demonstrated intense occupation of near-shelf regions that was punctuated by lengthy offshore excursions. Thus, perhaps the combination of favorable growth energetics associated with warm overwintering habitat (relative to overwintering at temperate latitudes) and food availability drive southerly movements away from temperate latitudes for winter and the mesopelagic occupation in (sub)tropical waters observed here. However, further work is needed to test the role of energetics and food resources as drivers of basking shark migrations.

Movement patterns of tagged basking sharks may also be associated with reproduction (Skomal et al., 2009). Basking sharks are commonly observed along the northeastern US 


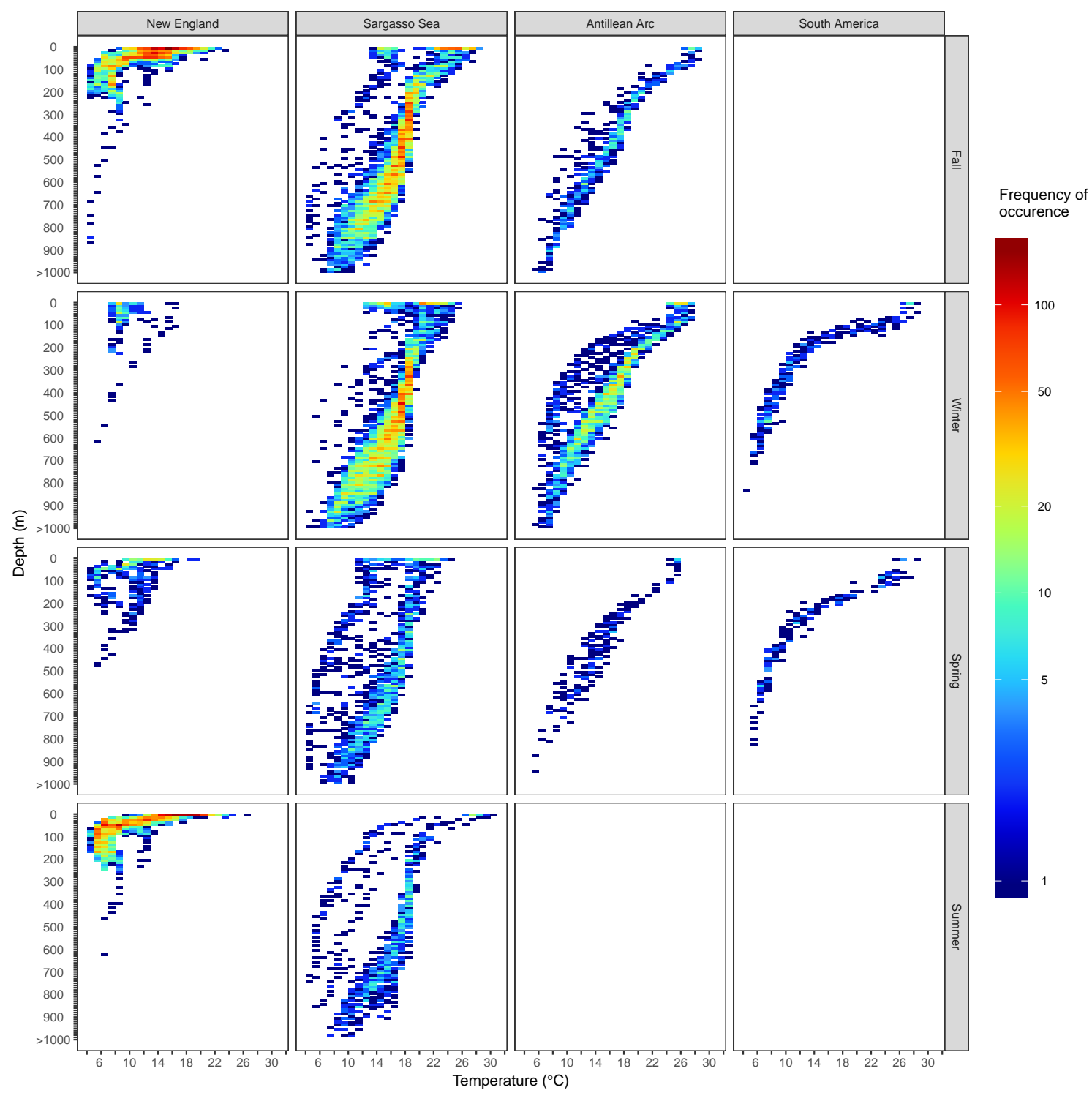

Figure 3-7: Vertical habitat envelopes of basking sharks. Temperature and depth data are binned every $1^{\circ}$ and $25 \mathrm{~m}$, respectively. Depths deeper than $1000 \mathrm{~m}$ are added to the last bin. The bounds for each region are shown as boxes in Figure 3-1. Note the color bar is on a log scale. Summary statistics for each region and season are shown in Table 3-2, and blank panels indicate no data was collected for that region-season combination.

during summer, presumably to forage; however, mating may also occur during this period while sharks are aggregated and potential courtship behavior has been observed (Wilson, 2004). Subsequent movements into the tropical Atlantic and occupation of mesopelagic depths may be a predator avoidance or parturition strategy as these environments are characterized by mild, stable conditions. This may further explain the lack of observations of pregnant females despite prolonged coastal fisheries in the NEA (Sims, 2008). Thus, while we did not observe significant differences in movement between sexes, the females that un- 
dertake long-range southerly migrations may be exploiting stable environmental conditions for gestation and parturition, and the stable habitat and relative lack of predators may provide suitable nursery habitat for neonates. The presence of $<2.5 \mathrm{~m}$ TL basking sharks in the Gulf of Mexico during spring (Hoffmayer et al., 2011) lends some support for this hypothesis as it suggests that parturition is occurring during winter months in tropical or subtropical waters. The wide variation in movement patterns $\left(>50^{\circ}\right.$ range in latitude) suggests these migrations were not driven by a localized mating event somewhere in the Atlantic. Unfortunately, we were unable to sex a significant portion of tagged individuals in this study due to tag application methods, and the limited sample size of sexed individuals indicates no difference in movements between sexes that may further clarify reproductive hypotheses.

Highly variable dive behavior, including extended forays away from the photic zone, exhibited by basking sharks made traditional light-based geolocation difficult in our study. Thus, we employed a recent advance (based on extensive work by Pedersen et al., 2008, $2011 b$ ) in geolocation analysis methods to supplement missing light data with other forms of data recorded on the tag (Braun et al., 2018a, Chapter 2). Depth-temperature profiles, in particular, provided substantially more information to be used for geolocation than light and SST data used in traditional geolocation approaches. These profiles provided observations that were used for geolocation when tagged individuals were away from the surface and the tags couldn't collect light and SST metrics. In addition, the profile data yielded diagnostic depth-temperature profiles that can be compared to modeled or in situ oceanographic data for reducing geolocation error (Braun et al., 2018a, Chapter 2). By using the high-resolution $\left(0.08^{\circ}\right)$ HYCOM reanalysis product, we were able to leverage the synoptic daily coverage of an oceanographic model that incorporates available in situ data to improve geolocation estimates. While previous tracking studies have shown some problems with the HYCOM reanalysis product being used to construct tracks through the Gulf Stream eddy field (Braun et al., 2018a, Chapter 2), the majority of basking sharks in this study moved latitudinally and spent relatively little time in the most dynamic regions of the NWA.

Model outputs also indicated a higher likelihood of "resident-like" movements in productive shelf habitats around New England and off the Antilles and South America. It is likely these restricted movements are indicative of foraging in these relatively productive shelf habitats (Mourato et al., 2014). In contrast, migratory movements $\left(4 \mathrm{~m} \cdot \mathrm{s}^{-1}\right)$ were more likely in pelagic waters, including during overwintering in the Sargasso Sea. Because of model formulation, the higher speeds that we classified as "migratory" may also be more likely, overall, due to the scale at which the observation likelihoods are formulated. For instance, if tag-based SST corresponds to remotely sensed SST over a broad area (e.g. Sargasso Sea), we may expect migratory behavior to be more likely than the resident behavior that would result from more constrained likelihoods (e.g. tag-based SST matching more closely 
Table 3-2: Summary statistics for vertical habitat envelopes in Figure 3-7 by region and season.

\begin{tabular}{|c|c|c|c|c|c|}
\hline & & New England & Sargasso Sea & Antillean Arc & South America \\
\hline \multirow[t]{5}{*}{ Fall } & $\mathrm{SST}$ & $15.6(9.3-25)$ & $24.5(13.4-29.4)$ & $27.5(26.3-28)$ & \\
\hline & Min Z & $5.6(0-240)$ & $192.9(0-876)$ & $201(0-932)$ & \\
\hline & Max Z & $72(8-1096)$ & $816(16-1200)$ & $760(284-1072)$ & \\
\hline & Min $\mathrm{T}$ & $8.4(4.2-16.4)$ & $11.2(4.6-18.8)$ & $9.4(5.8-17.6)$ & \\
\hline & $\mathrm{N}$ & 3813 & 7116 & 744 & \\
\hline \multirow[t]{5}{*}{ Winter } & $\mathrm{SST}$ & $9.2(7.4-15.6)$ & $21.9(14.6-24.5)$ & $26(24.6-27.2)$ & $28(27.7-28.8)$ \\
\hline & Min Z & $4.9(0-64)$ & $276.3(0-868)$ & $168.8(0-536)$ & $121.6(0-488)$ \\
\hline & $\operatorname{Max} \mathrm{Z}$ & $168(40-616)$ & $840(8-1448)$ & $712(196-1328)$ & $532(236-832)$ \\
\hline & Min $\mathrm{T}$ & $8(5.6-11)$ & $11.4(4.2-19.2)$ & $9.2(4.6-18.6)$ & $7(4.6-11.6)$ \\
\hline & $\mathrm{N}$ & 262 & 6636 & 2691 & 314 \\
\hline \multirow{5}{*}{ Spring } & SST & $13.9(7.7-16.7)$ & $20.5(10.4-24.3)$ & $25.4(24.9-25.6)$ & $27(24.6-28.8)$ \\
\hline & Min Z & $7.1(0-152)$ & $205(0-792)$ & $179.2(0-424)$ & $99.8(0-420)$ \\
\hline & Max Z & $72(24-472)$ & $862(272-1200)$ & $664(440-944)$ & $560(264-820)$ \\
\hline & $\operatorname{Min} \mathrm{T}$ & $7.8(4.2-13.5)$ & $8.7(4.4-18.6)$ & $9(5.8-11.4)$ & $6.8(5.2-13)$ \\
\hline & $\mathrm{N}$ & 479 & 1641 & 197 & 196 \\
\hline \multirow[t]{5}{*}{ Summer } & $\mathrm{SST}$ & $18.1(12-25.8)$ & $26.7(25-29.9)$ & & \\
\hline & Min Z & $8.9(0-352)$ & $303.8(0-788)$ & & \\
\hline & Max Z & $72(12-624)$ & $776(284-1040)$ & & \\
\hline & Min $\mathrm{T}$ & $7(4.4-15.7)$ & $12.8(4.7-19)$ & & \\
\hline & $\mathrm{N}$ & 3860 & 976 & & \\
\hline
\end{tabular}

$\overline{\text { Reported values are formatted as median(minimum-maximum) for sea surface temperature (SST), }}$ minimum daily depth (Min Z), maximum daily depth (Max Z) and minimum daily temperature (Min T). Temperatures are ${ }^{\circ} \mathrm{C}$ and depths are in meters. Sample sizes $(\mathrm{N})$ indicate total number of data points (not individual profiles) and are shown for each region-season combination. Blank combinations in the table indicate no data was collected for that combination. Note these data were restricted to the spatial areas of interest as shown in Figure 3-1 and may not exactly match reported statistics in the text which included all data. 
to a confined region). While this approach is significantly more computationally-intensive than traditional light-based geolocation approaches, comparing tag data directly to in situ and/or modeled oceanographic profiles from the same time frame results in a more realistic representation of shark movements and the oceanographic environment they inhabit.

The basking shark tracks documented here represent the largest scale movements reported for basking sharks, including one individual's estimated track distance covering $>17,000 \mathrm{~km}$, and the deepest dive recorded by a basking shark $(1504 \mathrm{~m})$. The observed tracks further expand the known range of basking sharks reported by Skomal et al. (2009). We recorded 3 individuals making transequatorial migrations yet no tagged individuals made significant longitudinal movements toward the NEA. North-south movements were, therefore, much more common in the portion of the NWA population sampled here than east-west movements that may, in turn, limit the exchange of genetic material between the NWA and NEA. In contrast, Gore et al. (2008) found that one of two satellite-tagged basking sharks moved from the Isle of Man to the eastern coast of Newfoundland in less than 3 months. In addition, there is little evidence for genetic structuring of basking sharks in the Atlantic Hoelzel et al. (2006), suggesting sufficient connectivity to at least maintain panmixia between NEA and NWA populations.

\subsection{Conclusion}

The current reliance on light levels for geolocation of many marine fishes renders geolocation impossible when tagged individuals spend significant time below the euphotic zone. Tagged sharks in this study spent significant time at mesopelagic depths, particularly during winter, at which light levels were too low for geolocation. We supplemented light-based geolocation with position estimates generated by matching depth-temperature profiles collected by the sharks' tags to in situ or modeled oceanographic profiles. Our approach provided considerably more information on movement patterns than are typically available from PSAT data with limited light-level information, providing a valuable method for studying marine species that do not frequent the euphotic zone. The resulting basking shark tracks demonstrated large-scale movements up to over $17,000 \mathrm{~km}$ from Cape Cod to southern Brazil, winter residency in New England waters, and a range of behaviors in between. Most individuals exhibited seasonal movements into the Sargasso Sea during winter and multiple deployments of sufficient duration captured the return migration to Cape Cod the subsequent summer. Basking sharks in this study traversed multiple distinct water masses through the western Atlantic and exhibited basin-scale movements that warrant international cooperation for

adequate management of this species. Winter habitat use was characterized by occupation of mesopelagic waters at low latitudes during which individuals often left the surface for months at a time. This cryptic deep-water overwintering provides impetus for further study 
of this poorly understood species. 
THIS PAGE INTENTIONALLY LEFT BLANK 
Chapter 4

\section{DETERMINING OCEANOGRAPHIC ASSOCIATION, MOVEMENTS AND CONNECTIVITY OF SWORDFISH IN THE North Atlantic BY integrating EleCtronic TAGGING AND FISHERIES DATA}

The data used in this study is a collaborative effort among the follow co-authors: C.D. Braun, P. Gaube, P. Afonso, J. Fontes, G.B. Skomal, and S.R. Thorrold

All authors designed the study. G.B.S., P.A and J.F. conducted the tagging. C.D.B. performed the analysis with P.G. and S.R.T. C.D.B. wrote the paper with contributions and final approval from all authors.

The supplemental methods, figures, and tables for this chapter can be found in Appendix B. 


\subsection{Abstract}

Broadbill swordfish (Xiphias gladius) form the basis of commercial fisheries throughout the world's tropical and temperate oceans. Atlantic Ocean swordfish stocks appear to be recovering from years of overfishing, but other populations remain under significant fishing pressure. Management efforts for wide-ranging pelagic fishes, including swordfish, continue to be hindered by data gaps in the basic biology of exploited species. We addressed a significant knowledge gap in the movement ecology of Atlantic swordfish by deploying 20 pop-up satellite archival transmitting (PSAT) tags on individuals captured in the Azores and the Northwest Atlantic (NWA). Reporting tags transmitted low quality light and SST data, so we applied a recently developed geolocation approach that uses hydrographic profile data to improve geolocation accuracy. Resulting tracks from 17 individuals averaged $3,751 \mathrm{~km}$ (range 345-7,247) over 93 days (range 17-181). Fish tagged in the Azores primarily moved between the Azores archipelago and the Azores Front south of the islands. Movements of fish tagged in the NWA spanned $35^{\circ}$ of latitude from summer foraging grounds near the Grand Banks to winter habitats near the Antillean Arc. We also mined fisheries-dependent data from the International Commission for the Conservation of Atlantic Tunas (ICCAT) databases. We used ICCAT data from 15,101 conventional tag releases and 568 recaptures, as well as catch-per-unit effort (CPUE) data from $~ 135$ million and 1.7 billion hooks set by the U.S. and international longline fleets, respectively, to supplement the satellite tag data. Movements and displacement of satellite and conventional tags indicated complex stock structure within the North Atlantic. Across all three datasets, swordfish exhibited remarkable physiological capabilities by occupying a $\sim 25^{\circ} \mathrm{C}$ SST range in primarily oligotrophic waters and frequented active front boundaries. These data also suggested swordfish occupied eddies across the study region and satellite- and conventional-tagging data suggest a possible preference for anticyclones, although the influence of fishing effort cannot be ruled out. Generalized additive modeling corroborated the eddy results and also highlighted the importance of geographic location influencing CPUE, as well as SST, chlorophyll and minor contributions from other predictor variables. Our work demonstrates the utility in synthesizing results from fishery-independent electronic tag data and fisheries datasets to improve understanding of oceanographic influences on swordfish.

\subsection{Introduction}

Swordfish have the broadest geographical distribution of all billfishes (Braun et al., 2015a) and are found worldwide from $45^{\circ} \mathrm{S}$ to $45^{\circ} \mathrm{N}$ (Palko et al., 1981). This species is the target of the most valuable and ubiquitous commercial billfish fishery in the world (FAO, 2012) which has historically led to the overexploitation of swordfish stocks. Despite their economic 
value, swordfish now appear to be adequately managed throughout the Atlantic, with North Atlantic stocks exhibiting recovery after years of overfishing (Neilson et al., 2013; Collette et al., 2011). The Atlantic swordfish population is currently managed as three stocks (North, South and Mediterranean) based on conventional (Garcia-Cortes and Quintans, 2003) and satellite (Neilson et al., 2009; Abascal et al., 2015) tagging and analyses of population genetics (Bremer et al., 1996; Alvarado Bremer et al., 2005; Smith et al., 2015). However, recent genetic evidence suggests that the boundary between north and south stocks may require revision (Smith et al., 2015), and some tagging studies have observed potential structure within the North Atlantic (Neilson et al., 2009; Abascal et al., 2015).

Most of the existing research on Atlantic swordfish is driven by stock assessment needs. Nearly all biological and ecological parameters are derived from the relatively extensive work in the NWA compared to the rest of the Atlantic basin. Tagging studies indicate North Atlantic swordfish exhibit largely North-South movements driven by contrasting ocean regimes for feeding and reproduction (Sedberry and Loefer, 2001; Neilson et al., 2009; Abascal et al., 2015 ) in which they move from productive foraging grounds in the far north during summer to warm tropical and subtropical waters in winter to spawn and to promote larval growth (Arocha and Lee, 1995). During these movements, they exhibit extreme physiological versatility in which they regularly experience a $15^{\circ} \mathrm{C}$ daily temperature range (Abascal et al., 2015) during regular diel vertical migrations (Carey and Robison, 1981; Lerner et al., 2013) to the deep scattering layer (Carey, 1990; Josse et al., 1998).

Reliable stock assessments, including habitat-based vulnerability models and CPUE corrections (Hinton and Nakano, 1996; Bigelow and Maunder, 2007), and detecting effects of climate changes (Schirripa et al., 2016) rely on a thorough understanding of the oceanographic characteristics driving animal behavior. For instance, physical-biological interactions that occur at the oceanic mesoscale $(\mathcal{O}(10-100 \mathrm{~km})$; McGillicuddy, 2016) and submesoscale $(\mathcal{O}(1$ $\mathrm{km}$ ); Flierl and McGillicuddy, 2002; Lévy et al., 2012) are known to influence pelagic fishes (Teo and Block, 2010; Seki Michael et al., 2002; Godø et al., 2012), including swordfish (Podestá et al., 1993; Scales et al., 2017). Eddies, currents and fronts are the main features that occur at these scales and influence animal movements (Hobday and Hartog, 2014). These features play a fundamental role in ocean dynamics, by structuring the physical and biological properties of the marine environment, and remain important foci for current physical-biological interactions research today (McGillicuddy, 2016).

The influence of oceanography on swordfish abundance and behavior has been investigated for decades, but these approaches have relied heavily on fisheries-dependent CPUE data and have yielded conflicting results. For example, Podestá et al. (1993) determined high swordfish CPUE more frequently occurred in the vicinity of fronts but showed no statistical relationship with frontal density or SST gradient, whereas Bigelow et al. (1999) found a positive relationship between swordfish CPUE and several front metrics. In addition, while 
$>90$ satellite archival tags have been deployed on swordfish in the North Atlantic (Braun et al., 2015a), geolocation error has largely precluded quantitative analyses of horizontal movements in the context of oceanography. As such, oceanographic drivers of swordfish movements remain poorly understood. Here, we analyze a fisheries-independent satellite tagging program on swordfish in the North Atlantic. We then combine high-resolution satellite tag data with inference from $>15,000$ conventional tag deployments and CPUE data from nearly 2 billion longline hooks to synthesize oceanographic drivers, movements and connectivity of swordfish in the North Atlantic.

\subsection{Materials and methods}

\subsubsection{Satellite tagging}

We deployed 20 pop-up satellite archival transmitting (PSAT) tags (17 miniPAT, 3 MK10AF; Wildlife Computers Inc., Redmond, WA, USA) on swordfish (Xiphias gladius) in the North Atlantic between 2009-2014, including 8 near the Grand Banks, 5 in the southern Sargasso Sea and 7 around the Azores (Table 4-1). Tags logged depth, temperature, and light level data every 10 seconds (Mk10-AF) or 15 seconds (miniPAT) to onboard memory. All tags recorded light data for geolocation purposes, and the Mk10-AF tags housed a Fastloc GPS receiver for acquiring high-resolution location information. Swordfish in the Azores were caught on rod and reel and brought alongside or onto the tagging vessel for tag placement. NWA swordfish were caught by a commercial longline vessel, brought alongside the boat and tagged if judged to be in good condition by the vessel captain. Weight and lower jaw fork length (LJFL) were estimated by the tagging team. Tags were applied into the dorsal musculature near the base of the dorsal fin using a titanium plate or nylon umbrella dart as an anchor. Tags were released from the swordfish using a corrosive burn wire at pre-programmed dates during tag deployment (range of programmed deployment duration 90-181 days). After the tags released and floated to the surface, summarized data were transmitted to Argos satellites until battery failure. Transmitted data were decoded with manufacturer software (WC-DAP 3.0, Wildlife Computers, Inc., Redmond, WA). All data from tags that released prematurely after less than 10 days were removed from all analyses. All further analyses were conducted in R (R Development Core Team, 2015), and a KruskalWallis test was used to compare density distributions. For visualization purposes, tag-based depth-temperature profile plots were constructed by computing a weighted least-squares regression (LOESS) using half-power cutoffs of \pm 5 days and $150 \mathrm{~m}$ from all daily depthtemperature data ( $\mathrm{R}$ code available on request). This research was performed in accordance with the Woods Hole Oceanographic Institution's Animal Care and Use Committee (IACUC) protocol \#16518. 


\section{Geolocation methods}

Most probable tracks for PSAT-tagged swordfish were constructed using the HMMoce package (Braun et al., 2018a, Chp. 2) for R (R Development Core Team, 2015). This approach uses light-levels, sea surface temperature, depth-temperature profiles and bathymetry recorded by archival tags, with observations and model outputs, to construct likelihoods of the tagged individual's movements. Likelihoods are convolved in a gridded hidden Markov model that computes posterior probability distributions to estimate the most likely state (position and behavior) of the animal at each time point.

Observation-based likelihoods were derived from remotely-sensed SST, light-based longitude and depth-temperature profile data collected by the tags, using four separate likelihood calculations: 1) An SST likelihood was generated for tag-based SST values integrated according to an error term $( \pm 1 \%)$ and compared to remotely-sensed SST from daily optimallyinterpolated sea surface temperature (OI-SST, $0.25^{\circ}$ resolution) fields (Reynolds et al., 2007; Banzon et al., 2016). 2) Light-based longitude likelihood was derived using estimates of longitude from GPE2 software (Wildlife Computers, Inc.) that facilitated visual checking of light curves. 3) Depth-temperature profiles recorded by the tag were compared to daily reanalysis model depth-temperature products from the HYbrid Coordinate Ocean Model (HYCOM, $0.08^{\circ}$ resolution; Bleck, 2002; Chassignet et al., 2007) at standard depth levels available in these products. Individual likelihood surfaces for each depth level were then multiplied together for an overall profile likelihood at that time point. 4) Ocean Heat Content $(\mathrm{OHC})$ was obtained by integrating the heat content of the water column above the minimum daily temperature to the most shallow depth recorded by the tag for both the tag profiles and HYCOM fields (Luo et al., 2015; Braun et al., 2018a). Start and end locations were considered known in all cases and model runs.

The resulting observation likelihoods, in all possible pairwise and triplicate combinations, were convolved with two different movement kernels representing resident and migratory behavior "states" of tagged fish. For full details of the convolution, filtering and smoothing components of the model see Braun et al. (2018a) (Chapter 2). Parameter estimation was performed using an Expectation-Maximization algorithm following Woillez et al. (2016), and model selection used Akaike Information Criterion (AIC). Results from the final smoothing step of the selected model represent the posterior distribution of each state over time. Distributions were summed for each behavior state and time step to determine the most likely behavior state at each time. The mean of the daily distributions was used to calculate a most probable track. 
Table 4-1: Summary of swordfish tagged with PSATs in the North Atlantic Ocean. Est Wt = Estimated weight (kgs). Stage $=$ stage of maturity for juvenile (J), sub-adult (SA) and adult (A) swordfish. Tag types are miniPAT (mP) or FastLOC (fL). Region indicates where an individual was tagged and refers to the Grand Banks (GB), the Azores (AZ) or offshore from Florida in the southern Sargasso Sea (FL). Time-at-liberty (TAL) is in days. Distance (Dist.) is cumulative track distance in km. PDT, Light and SST = percent of deployment period with depth-temperature profiles (PDT), light and sea surface temperature (SST) data, respectively. Observation likelihoods (Obs.) indicate observations used in HMMoce to construct the most probable track: $\mathrm{L}=$ light-based longitude, $\mathrm{S}=$ sea surface temperature, $\mathrm{H}=\mathrm{HYCOM}$ depth-temperature profiles, $\mathrm{O}=$ integrated Ocean Heat Content, $\mathrm{F}=$ Fastloc GPS, DD=data deficient.

\begin{tabular}{|c|c|c|c|c|c|c|c|c|c|c|c|c|c|c|c|}
\hline ID & Tag Date & $\begin{array}{l}\text { Tag } \\
\text { Lat }\end{array}$ & $\begin{array}{l}\text { Tag } \\
\text { Lon }\end{array}$ & $\begin{array}{l}\text { Est } \\
W t\end{array}$ & Stage & Type & Region & TAL & $\begin{array}{l}\text { Pop } \\
\text { Lat }\end{array}$ & $\begin{array}{l}\text { Pop } \\
\text { Lon }\end{array}$ & Dist. & $\begin{array}{l}\text { PDT } \\
(\%)\end{array}$ & $\begin{array}{l}\text { Light } \\
(\%)\end{array}$ & $\begin{array}{l}\text { SST } \\
(\%)\end{array}$ & Obs. \\
\hline 98751 & 2009-12-02 & 38.44 & -28.52 & 11.0 & $\mathrm{~J}$ & $\mathrm{mP}$ & $\mathrm{AZ}$ & 17 & 32.92 & -28.18 & 773 & 88 & 47 & 82 & LSH \\
\hline 104668 & 2011-10-21 & 38.41 & -28.51 & 19.5 & $\mathrm{~J}$ & $\mathrm{mP}$ & $\mathrm{AZ}$ & 35 & 27.17 & -30.92 & 2590 & 91 & 9 & 89 & LSH \\
\hline 104670 & 2011-10-21 & 38.44 & -28.56 & 23.5 & $\mathrm{~J}$ & $\mathrm{mP}$ & $\mathrm{AZ}$ & 26 & 31.9 & -30.89 & 902 & 88 & 85 & 42 & LSH \\
\hline 98721 & 2011-10-26 & 38.44 & -28.54 & 12.0 & $\mathrm{~J}$ & $\mathrm{mP}$ & $\mathrm{AZ}$ & 38 & 38.55 & -28.91 & 345 & 95 & 5 & 8 & LSH \\
\hline 98722 & $2011-10-26$ & 38.43 & -28.53 & 11.0 & $\mathrm{~J}$ & $\mathrm{mP}$ & $\mathrm{AZ}$ & 150 & 38.17 & -28.56 & 5335 & 60 & 24 & 81 & LSH \\
\hline 104671 & 2011-11-15 & 37.84 & -27.17 & 17.5 & $\mathrm{~J}$ & $\mathrm{mP}$ & $\mathrm{AZ}$ & 150 & 37.84 & -27.17 & 4816 & 71 & 33 & 91 & LSH \\
\hline 104672 & 2011-11-15 & 37.84 & -27.17 & 10.0 & $\mathrm{~J}$ & $\mathrm{mP}$ & $\mathrm{AZ}$ & 55 & 33.54 & -28.53 & 2357 & 96 & 38 & 98 & LSH \\
\hline 100976 & $2010-11-25$ & 28.20 & -66.92 & 50.0 & SA & $\mathrm{mP}$ & FL & 24 & 20.27 & -65.05 & 979 & 92 & 0 & 92 & $\mathrm{LH}$ \\
\hline 100980 & 2010-11-30 & 28.34 & -67.52 & 68.2 & $\mathrm{~A}$ & $\mathrm{mP}$ & FL & 90 & 30.84 & -75.17 & 3828 & 94 & 1 & 73 & LSH \\
\hline 100978 & 2011-01-10 & 27.57 & -67.92 & 45.5 & $\mathrm{SA}$ & $\mathrm{mP}$ & FL & DD & & & & & & & $\mathrm{DD}$ \\
\hline 95975 & 2011-01-20 & 24.61 & -68.58 & 56.8 & $\mathrm{SA}$ & $\mathrm{mP}$ & FL & 181 & 38.93 & -53.2 & 6782 & 83 & 9 & 81 & LSH \\
\hline 95983 & 2011-01-31 & 25.90 & -69.53 & 56.8 & $\mathrm{SA}$ & $\mathrm{mP}$ & FL & DNR & & & & & & & \\
\hline 110490 & 2011-09-15 & 43.67 & -48.81 & 81.8 & A & $\mathrm{mP}$ & GB & 90 & 30.29 & -63.63 & 4449 & 79 & 39 & 96 & LSH \\
\hline 110491 & 2011-09-15 & 43.65 & -48.82 & 81.8 & $\mathrm{~A}$ & $\mathrm{mP}$ & GB & 180 & 12.35 & -60.04 & 6914 & 36 & 33 & 64 & LSH \\
\hline 110497 & 2011-09-19 & 43.94 & -48.57 & 65.9 & $\mathrm{~A}$ & $\mathrm{mP}$ & GB & 90 & 18.7 & -76.16 & 4869 & 72 & 21 & 70 & $\mathrm{LH}$ \\
\hline 110498 & 2011-09-22 & 43.90 & -48.40 & 100.0 & $\mathrm{~A}$ & $\mathrm{mP}$ & GB & 90 & 32.38 & -59.38 & 2718 & 74 & 14 & 83 & LSH \\
\hline 110496 & 2011-10-17 & 44.57 & -46.90 & 61.4 & $\mathrm{~A}$ & $\mathrm{mP}$ & GB & 149 & 34.95 & -50.37 & 7247 & 99 & 32 & 97 & LSO \\
\hline 106788 & $2013-09-27$ & 46.69 & -45.80 & 90.9 & $\mathrm{~A}$ & $\mathrm{fL}$ & GB & 180 & 20 & -74.28 & 6714 & 27 & 1 & 3 & LSH \\
\hline 106795 & $2013-09-27$ & 46.48 & -45.56 & 90.9 & $\mathrm{~A}$ & $\mathrm{fL}$ & GB & 40 & 31.03 & -39.68 & 2146 & 95 & 28 & 70 & FLSH \\
\hline 106797 & 2013-09-27 & 46.62 & -45.64 & 68.2 & $\mathrm{~A}$ & $\mathrm{fL}$ & GB & DNR & & & & & & & \\
\hline
\end{tabular}




\subsubsection{Fisheries data}

To evaluate movements of satellite-tagged fish in relation to the swordfish longline fishery in the North Atlantic, we obtained fisheries data (Task II catch-effort; version Nov. 2016) from the ICCAT Secretariat (http://www.iccat.int/en/accesingdb.htm). We extracted longline (LL) data for the years 1990-2010 in the North Atlantic west of $20^{\circ} \mathrm{W}$ at two different spatial resolutions: 1) $1^{\circ} \times 1^{\circ}$ for the U.S. fleet and 2) $5^{\circ} \times 5^{\circ}$ resolution data available for the international fleets from Brazil, China, Cuba, Greece, Japan, Korea, Libya, Mexico, Spain, U.K., Uruguay. Longline catch data were used to calculate mean catch per unit effort (CPUE; number of fish per 1000 hooks) in each grid cell. CPUE data resulting in > 200 fish per 1000 hooks were considered an error and removed from the dataset. We also obtained conventional tag data from the ICCAT Secretariat tag database for swordfish in the North Atlantic from 1940-2016.

\subsubsection{Oceanographic data}

In order to characterize associations between swordfish and the oceanographic environment, we acquired remote sensing and climatological data from multiple sources. We used a monthly composite of remotely-sensed Chlorophyll-a from SeaWiFS (nominal resolution of $9 \mathrm{~km})$ and bathymetry from Smith and Sandwell (0.0167 ${ }^{\circ}$ resolution, version 11.1$)$. Distance to the $200 \mathrm{~m}$ bottom contour was calculated from bathymetry. Daily sea surface temperature (SST) data were acquired from the HYCOM reanalysis product (global $0.08^{\circ}$ daily resolution) due to the broad temporal coverage of the swordfish data. Although the HYCOM data were modeled SST, these data were comparable to remotely-sensed SST from Multi-scale Ultra-high Resolution (MUR) data (global $0.01^{\circ}$ daily resolution) during the shorter period in which both were available (Fig. B-1). Fronts were detected in daily HYCOM SST fields using a threshold algorithm (Belkin and O'Reilly, 2009) implemented in the boaR package (Galuardi, 2017) for $\mathrm{R}$ and composited to monthly front frequency using thermal front magnitude $>0.1^{\circ} \mathrm{C} \cdot \mathrm{km}^{-1}$. We used monthly global estimates of climatological isothermal $\left(\Delta T=0.2^{\circ} \mathrm{C}\right)$ mixed layer depth (de Boyer Montégut et al., 2004), and a climatological representation of the north wall of the Gulf Stream defined as the position of the $15^{\circ} \mathrm{C}$ contour at $200 \mathrm{~m}$ in climatological annual mean temperature from the World Ocean Atlas (2013, 0.25 global) (Fuglister, 1963; Gaube and McGillicuddy, 2017).

Monthly mean sea surface height ( $\mathrm{SSH}$, global $0.25^{\circ}$ ) was computed from AVISO sealevel anomaly (SLA) data by removing the result of high-pass filtering the SLA fields using a $20^{\circ} \times 10^{\circ}$ (lon $\mathrm{x}$ lat) $2 \mathrm{D}$ LOESS smoother to remove effects of seasonal heating and cooling (Chelton et al., 2011) as:

$$
S S H=S L A-\langle S L A\rangle
$$


where the $<>$ operator indicates spatial smoothing. We derived a monthly climatology for deep scattering layer depth $\left(Z_{D S L}\right)$ based on a stepwise multiple linear regression from Bianchi et al. (2013):

$$
Z_{D S L}=398-0.56 \cdot \Delta O_{2}-115 \cdot \log (C H L)+0.36 \cdot M L D-2.4 \cdot \Delta T
$$

where $\Delta \mathrm{O}_{2}$ and $\Delta T$ were differences between $0-25 \mathrm{~m}$ and $150-500 \mathrm{~m}$ in monthly climatological mean oxygen and temperature data, respectively, from the World Ocean Atlas 2013 (Garcia et al., 2014; Locarnini et al., 2013). Finally, we used the monthly composite of chlorophyll data $(\mathrm{CHL})$ from SeaWiFS and the mixed layer depth climatology described above $(M L D)$.

To quantify associations between swordfish and mesoscale eddies, we collocated conventional tag data to the Mesoscale Eddy Trajectory Atlas from Aviso that contains daily tracks of coherent mesoscale structures (CMS) based on maps of sea surface height (Chelton et al., 2011). We developed a meander filter (similar to Gaube and McGillicuddy, 2017) for the Gulf Stream and Gulf of Mexico region in which we defined a mask $\pm 1^{\circ}$ north and south from the $40 \mathrm{~cm}$ ADT contour that approximated the north wall of the Gulf Stream and the Loop Current in the Gulf of Mexico to distinguish between mesoscale eddies and meanders. The mask was extended to $2^{\circ}$ south of the ADT contour in the Gulf Stream to eliminate anticyclonic meanders. Those features in which the core of the CMS was within the meander mask were considered meanders and removed from the remainder of the analysis.

We visually compared swordfish tracks and depth-temperature profile data from the PSAT tags to the SSH fields and tracked eddies to generate a qualitative comparison of eddy use from these data. Eddy occupation was quantified using a subset of the conventional tag data to include those swordfish release and recapture positions that were in regions of known mesoscale activity, including the Gulf Stream, Sargasso Sea and Gulf of Mexico, in water deeper than $2000 \mathrm{~m}$ and that corresponded to the temporal limits of the eddy tracking dataset (Jan 1993 - Sept 2016). These locations were collocated to the nearest eddy identified in the eddy atlas, after applying the meander filter in the Gulf Stream and Gulf of Mexico. To assess differences in the distribution of swordfish in anticyclonic and cyclonic eddies, we constructed histograms of swordfish location as a function of normalized radial distance $(r)$, defined as the geographic distance $(d)$ normalized by the speed-based eddy radius scale $L_{s}$. The resulting histogram values were then normalized by the area of each radial annulus defined by the histogram bin size and the distance $r$ from the eddy center. To determine if swordfish were more likely to be associated with the core, interior or periphery of eddies of either polarity, we defined eddy subregions by the normalized distance $(r)$ from the eddy SSH extremum, where the inner-core is defined as $r \leq L_{s} / 2$, the outer core as $L_{s} / 2<r \leq L_{s}$ and the eddy interior includes both the inner and outer core $r \leq L_{s}$. The eddy periphery is defined as $L_{s}<r \leq 2 L_{s}$ and the area outside of an eddy as $r>2 L_{s}$ 
(see Fig. 2 in Gaube et al., 2017). To assess the role of passive advection and the relative spatial composition of the sub-regions of eddies of each polarity, we also collocated a surface drifter dataset. The drifter dataset was acquired from NOAA's Atlantic Oceanographic and Meteorological Laboratory, and we used all drifters within each study region for a 5-year period (2005-2009) (Fig. B-2).

\subsubsection{GAM model formulation}

Oceanographic variables were used to develop generalized additive models (GAMs) to quantify the relationship between the physical environment and swordfish CPUE from the U.S. longline fleet. The oceanographic variables considered in model development were: SST $\left({ }^{\circ} \mathrm{C}\right)$, SSH $(\mathrm{m})$, chlorophyll-a $\left(m g \cdot m^{-3}\right)$, climatological DSL depth $(\mathrm{m})$, climatological MLD $(\mathrm{m})$, thermal front range $\left({ }^{\circ} \mathrm{C}\right)$ and frequency $\left(\right.$ days $\left.\cdot \mathrm{month}^{-1}\right)$, bottom depth $(\mathrm{m})$, distance to climatological position of the Gulf Stream $(\mathrm{km})$ and distance to the $200 \mathrm{~m}$ depth contour. Due to the summarized nature of the catch data (monthly, $1^{\circ}$ grid), oceanographic variables were all summarized as necessary to match this spatial and temporal scale. Front range was collinear with SST and removed. Bathymetry was collinear with the distance to the 200 $\mathrm{m}$ contour so standard deviation of bathymetry within a grid cell was used instead. Zero values were removed from the CPUE data to avoid zero-inflation in the model (Zuur et al., 2009; Maunder and Punt, 2004), effectively allowing us to model oceanographic drivers of varying catch (rather than presence-absence).

GAMs were constructed with the mgcv package (Wood, 2006) in R using the negative binomial family with a log-link function. Explanatory variables (except month) were modeled as continuous and smoothed by functions chosen automatically using the mgcv package and checked manually. The base model, after adjusting for collinearity, included 12 predictor variables for which the model was formulated as:

$$
C P U E=S(\text { Location })+\text { Month }+f_{1}\left(X_{1}\right)+f_{2}\left(X_{2}\right)+\ldots+f_{j}\left(X_{j}\right)
$$

where $S$ is a smoothing function for location (longitude, latitude); $f_{1} \ldots f_{j}$ are smoothing functions for environmental variable $1: j ; X_{1} \ldots X_{j}$ are values of the environmental variable $j$. Each smoothing function, with the exception of location, was permitted three degrees of freedom to minimize overfitting (Rooker et al., 2012). A stepwise backwards selection approach using Akaike Information Criterion (AIC) was used to select the final model. Predictor variables that were statistically significant at $p \leq 0.05$ were retained during selection and models were evaluated by checking diagnostic plots on fitted data and residuals (Fig. B-3). 

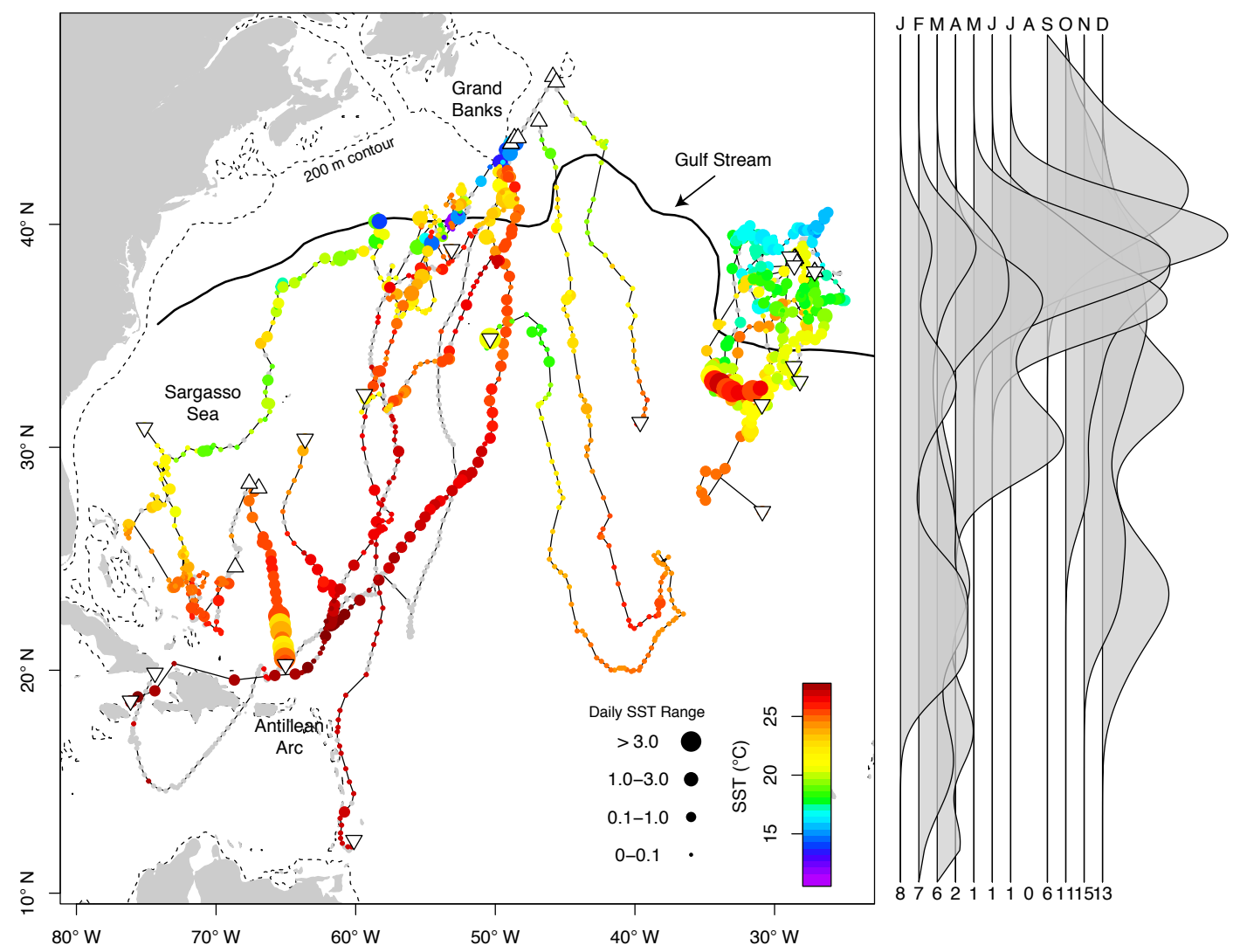

Figure 4-1: Movements of satellite-tagged swordfish in the North Atlantic. Most probable track estimates (left panel) for swordfish in which point color indicates tag-measured sea surface temperature (SST), and point size indicates the range of daily SST measured. Upward and downward pointing triangles represent release and pop-up positions, respectively. Dashed point and line contours represent the mean Gulf Stream position (see methods) and the $200 \mathrm{~m}$ bathymetric contour, respectively. Right panel shows monthly density distribution of satellite-tagged swordfish by latitude. Upper letters indicate month and lower number labels indicate sample size of tagged individuals. 


\subsection{Results}

Seventeen of the 20 PSAT tags deployed in this study reported and transmitted data, 9 of which were shed prematurely. The 17 reporting tags were at liberty for an average 93 days (range 17-181), and one tag was physically recovered after 149 days at liberty (PTT 110496; Table 4-1). All individuals $(\mathrm{n}=7)$ tagged near the Azores were juveniles with a mean estimated weight of $15 \mathrm{kgs}$ (range 10-24 kgs). The largest individuals were adults tagged near the Grand Banks ( $\mathrm{n}=8)$ which averaged $80 \mathrm{kgs}$ (range 61-100 kgs), and those tagged in the western Atlantic were a mix of adult $(\mathrm{n}=1)$ and subadult $(\mathrm{n}=4)$ fish averaging $55 \mathrm{kgs}$ (range 46-68 kgs).

The conventional tag database contained 15,101 swordfish releases and 568 recaptures from 1940 to 2016. The ICCAT Task II database contained catch and effort data from $~ 135$ million hooks set by the US longline fleet from 1990-2009 gridded at $1^{\circ}$ spatial resolution. This effort resulted in catch of 1.2 million swordfish with a mean CPUE of 12 fish / 1000 hooks (range 0-200). Data were available for longline vessels from other flag countries that set 1.7 billion hooks in the North Atlantic from 1990-2009. Approximately 4 million swordfish were harvested during this time period with mean CPUE of 3.4 fish / 1000 hooks (range 0-72). Most of the reported non-US catch data came from the Japanese (52\%), Chinese $(23 \%)$ and Spanish $(22 \%)$ fleets.

\subsubsection{Geolocation of PSATs}

Varying amounts of each data type were obtained from the PSATs for estimating most probable tracks (Table 4-1). Sufficient light available for geolocation was generally low (mean $25 \%$ of deployment days, range $0-85 \%$ ). SST and depth-temperature profile (PDT) data were recorded for a higher percentage of deployment days than light data in all but one transmitted dataset, and on average comprised $72 \%$ and $79 \%$ of deployment days respectively (range 3-98\% for SST and range 27-99\% for PDT). Two (out of 3 deployed) Fastloc tags reported data, but only one (PTT 106795) reported two realistic GPS positions collected during the deployment.

\subsubsection{Horizontal movements}

Overall movements of PSAT-tagged swordfish ranged from 345 to $7,247 \mathrm{~km}$ in up to 181 days at liberty and were predominantly oriented north-south (Fig. 4-1). We found no significant relationship between fish size and displacement using linear regression, and we found no significant difference in movement rates among fish from the three different tagging regions. The PSAT-tagged adults in the NWA moved across a $35^{\circ}$ latitudinal range between temperate waters in the summer as far north as the Grand Banks and tropical or subtropical waters during winter from the Caribbean to coastal Florida $\left(10-30^{\circ} \mathrm{N}\right)$ (Fig. 4-1). 
Model selection favored HYCOM-based profile likelihoods in 16 of 17 track calculations. The remaining geolocation analysis leveraged OHC-based profile likelihoods to generate the most likely track estimate (Table 4-1). State-switching dynamics in HMMoce suggested the migratory behavior state occurred 3 times more often than did residency behavior (not shown) and dominated as individuals traversed the Sargasso Sea or while the individuals tagged in the Azores moved through the southern extent of the observed movements. In contrast, more tortuous, resident-like behavior was common along the Antillean Arc, the Azores archipelago and in the "loop" movement exhibited around $20^{\circ} \mathrm{N}$ and $40^{\circ} \mathrm{W}$ along the mid-Atlantic Ridge by an adult tagged on the Grand Banks (Fig. 4-1). These habitats were connected by long-range, relatively directed movements through the Sargasso Sea with the majority of more tortuous movements occurring in the Gulf Stream and from the Bahamas southeast along the Antillean Arc and southern Sargasso Sea (range of cumulative track distances 979-7,247 km).

Seven tags were deployed on adults near the Grand Banks in September and October, and, by mid-October, all had left the tagging region. In early November, six individuals had crossed the Gulf Stream moving southwest into tropical water $\left(\sim 28-30^{\circ} \mathrm{C}\right)$ south of $30^{\circ} \mathrm{N}$ by December. The remaining individual tagged on the Grand Banks (PTT 110491) moved into the Gulf Stream in early November and remained mostly along the north wall until late December. Overwintering habitat was centered around $20^{\circ} \mathrm{N}$ but spanned $30^{\circ}$ of longitude from the eastern Caribbean and the NE coast of Venezuela to as far east as the mid-Atlantic Ridge. The individual tagged on the Grand Banks that moved SE to the mid-Atlantic Ridge began a return migration to the north in mid-February, but this tag popped up prematurely in mid-March along the $20^{\circ} \mathrm{C}$ isotherm near $35^{\circ} \mathrm{N}$. A single tag from January deployments off Florida transmitted reliable data (PTT 95975). This dataset comprised the only spring movements observed in this study and indicated northward migration began around midApril arriving to the Gulf Stream by May where this fish remained through mid-July.

The juveniles and subadults tagged near the Azores exhibited relatively restricted movements, in both latitudinal range $\left(27-40^{\circ} \mathrm{N}\right)$ and overall distance $(345-5,335 \mathrm{~km})$, as compared to the larger fish tagged in the NWA. These individuals were tagged in late October and early November around $38^{\circ} \mathrm{N}$ and, in general, moved south into the warmer, Azores Front region at $\sim 32^{\circ} \mathrm{N}$. Of the 3 tags at liberty in January, 1 returned to the Azores in late December followed by a second that returned in mid-February. The third tag deployment ended in early January at $33^{\circ} \mathrm{S}$.

Seasonality observed in both conventional tag and catch data was similar to the movements by the satellite-tagged fish (Fig. B-4). In general, CPUE was highest along the Grand Banks, the central North Atlantic and around Bermuda in the summer and moved away from northern latitudes in the winter (Fig. 4-2). During winter and spring months, CPUE was focused from the Gulf of Mexico, east to the southeastern U.S., Caribbean Sea, and along 
the Antilles. Effort began returning north, particularly by the international (non-U.S.) fleet, in spring and most longline fishing effort returned northward to the Grand Banks and extended south to $\sim 20^{\circ} \mathrm{N}$ in the central North Atlantic by summer (Fig. 4-2). The majority of conventional tags were deployed and recovered along the eastern seaboard of the U.S. but spanned the North Atlantic from the Gulf coast of Texas to Europe and from the Amazon Delta to the Grand Banks. Conventional tag data exhibited similar north-south seasonality with winter efforts spread between $\sim 10-35^{\circ} \mathrm{N}$, moving almost exclusively north of $30^{\circ} \mathrm{N}$ and concentrated around $45^{\circ} \mathrm{N}$ during summer and fall, before moving south again starting in November (Fig. B-4).

We observed no overlap in movements by PSAT-tagged fish from the NWA and the Azores. Displacement of recovered conventional tags indicated limited connectivity between the NWA and NEA and between any of the ICCAT sampling zones, except between the Gulf of Mexico (BIL91) and the eastern U.S. (BIL92)(Fig. 4-3). The east-west division was most prominent around $30-35^{\circ} \mathrm{W}$ with $<3 \%$ of conventional tag recoveries (out of 568 total recovered tags) exhibiting movement across this boundary.

\subsubsection{Oceanographic association}

Swordfish exhibited significant physiological plasticity by occupying a SST range of $\sim 22^{\circ} \mathrm{C}$. Satellite-tagged individuals in the Azores occupied a significantly lower distribution of SST (mean 19.8, range $14.9-28^{\circ} \mathrm{C}$ ) than either NWA individuals (mean 23.8, range 10.8$28.1^{\circ} \mathrm{C}$; Kruskal-Wallis test, $p<0.001$ ) or conventional tag data (mean 23.3, range $7.2-32^{\circ} \mathrm{C}$; $p<0.001$ )(Fig. B-5). PSAT-tagged swordfish in the NWA primarily occupied oligotrophic waters with mean chlorophyll values of $0.13 \mathrm{mg} \cdot \mathrm{m}^{-3}$ (range $0.03-1.12$ ). Waters with chlorophyll values above $0.25 \mathrm{mg} \cdot \mathrm{m}^{-3}$ occurred exclusively north of the Gulf Stream or along the southern edge of the Grand Banks. The smaller individuals near the Azores occupied a more restricted chlorophyll range (mean 0.19 , range $0.05-0.92 \mathrm{mg} \cdot \mathrm{m}^{-3}$ ) but used higher chlorophyll environments more frequently than the adults. Conventional tag data indicated similar affinity for low chlorophyll environments (mean 0.24 , range $0.04-7.45 \mathrm{mg} \cdot \mathrm{m}^{-3}$ ) whereby only $2 \%$ of all conventional tag locations with available chlorophyll data were $>1 \mathrm{mg} \cdot \mathrm{m}^{-3}$ (Fig. B-5). Nearly all PSAT-based movements occurred within regions that exhibited ample dissolved oxygen at depth $\left(>3 \mathrm{~mL} \cdot \mathrm{L}^{-1}\right.$ at $\left.500 \mathrm{~m}\right)$; however, the individuals that moved into the eastern Caribbean $(n=1)$ and along the eastern side of the lesser Antilles $(n=1)$ likely experienced DO between 2.5-3 $\mathrm{mL} \cdot \mathrm{L}^{-1}$ at $500 \mathrm{~m}$ (not shown). The majority of movements from PSAT-tagged fish were relatively directed (e.g. less tortuous) through the open ocean but were punctuated by periods of orientation to dynamic topographic features such as shelf break habitats (Fig. 4-1). Conventional tagging data was also concentrated around strong bathymetric gradients, particularly along the continental shelf of the eastern U.S. (Fig. 4-3), and $45 \%$ of these fish were captured in water $<1000 \mathrm{~m}$. We found no significant relationship 

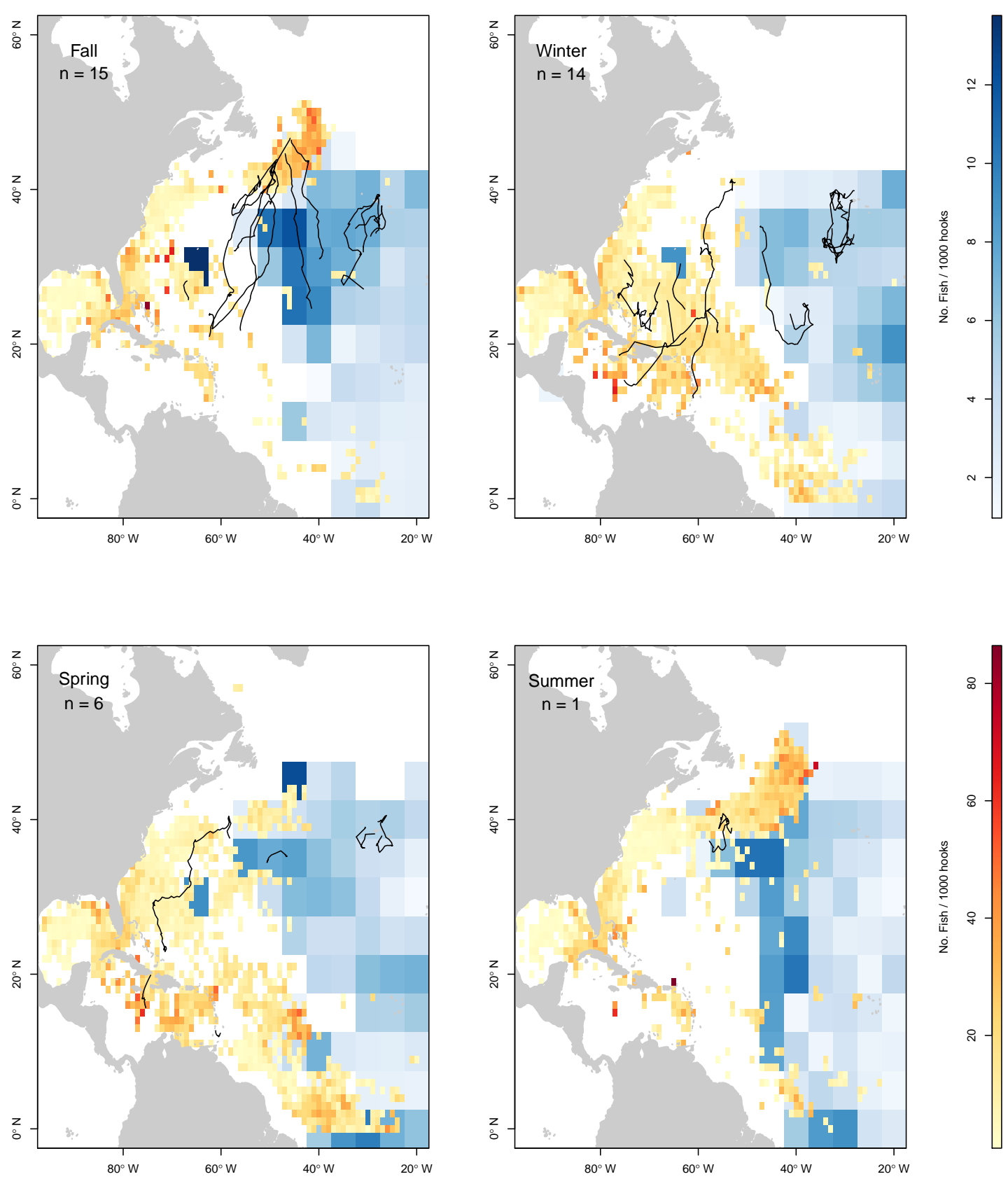

Figure 4-2: Seasonal distribution (Fall: Sep-Nov, Winter: Dec-Feb, Spring: Mar-May, Summer: Jun-Aug) of catch-per-unit effort (number of fish per 1000 hooks) of the longline swordfish fishery in the North Atlantic between 1990-2010 at $5^{\circ} \times 5^{\circ}$ resolution for the international fleet (blue color scale) and $1^{\circ} \times 1^{\circ}$ resolution for the U.S. fleet (red/yellow color scale). Tracks of tagged swordfish (this study) shown in black lines ( $\mathrm{n}=$ number of tagged individuals). 
between fish size and environmental characteristics (SST, bathymetry, chlorophyll) in the conventional tag data using linear regression.

Swordfish in this study were also associated with (sub)mesoscale features across the North Atlantic. Measured SST from the PSAT tags indicated that daily SST ranges regularly spanned $1-3^{\circ} \mathrm{C}$ around the Azores and in the Gulf Stream, and ranges $>3^{\circ} \mathrm{C}$ and as high as $7^{\circ} \mathrm{C}$ occured in the front-rich area north of Puerto Rico and in the Azores Front (Fig. $4-1$ ). In addition, $69 \%$ of the conventional tag locations (for which remote-sensing data is available; $\mathrm{n}=8627$ ) occurred in a region of weak front activity (magnitude $>0.1^{\circ} \mathrm{C} \cdot \mathrm{km}^{-1}$ ) while $35 \%$ were in areas characterized by stronger fronts (magnitude $>0.5^{\circ} \mathrm{C} \cdot \mathrm{km}^{-1}$ ). Ten and fifteen percent of conventional tag data occurred on or within $10 \mathrm{~km}$, respectively, of fronts $>1^{\circ} \mathrm{C} \cdot \mathrm{km}^{-1}$ in magnitude, which largely occurred along the shelf break of the eastern U.S. from northern Florida to the Grand Banks.

While the scale of uncertainty on PSAT track estimates is typically too large to reliably collocate swordfish movements to (sub)mesoscale features ( 100 km; Braun et al., 2018a, Chp. 2), a few PSAT-tagged individuals exhibited tracks and depth-temperature profile data that support their use of eddies. For example, during late March PTT 95975 occupied an open ocean anticyclonic eddy (ACE) offshore from Florida (Fig. 4-4A,C) and a cyclonic (CE) Gulf Stream eddy in early May (Fig. 4-4B,C). The ACE was characterized by depressed isotherms and warmer water at depth relative to the surrounding Sargasso Sea water (Fig. $4-4 \mathrm{C})$. In contrast, the more energetic Gulf Stream $\mathrm{CE}$ was $>6^{\circ} \mathrm{C}$ colder at $400 \mathrm{~m}$ than the ambient water and exhibited a core of cool $\left(\sim 16^{\circ} \mathrm{C}\right)$ water. Using conventional tag data, we were also able to quantify swordfish occupation of eddies in the Gulf of Mexico, Sargasso Sea and the Gulf Stream. In the Sargasso Sea, our data suggested no preference for eddies of either polarity and similar use of within-eddy structure relative to drifters in the same region (Fig. 4-5). In the Gulf of Mexico, conventional tags were deployed or recovered within ACEs nearly $70 \%$ more than in cyclones, most of which was focused within ACE cores. The opposite trend was observed for drifters in this same region in which nearly an order of magnitude more drifter locations were in cores of cyclones (Fig. 4-5). The Gulf Stream represented the most dynamic region for presence of mesoscale features in the study area. In this region, $2 \mathrm{x}$ more swordfish conventional tag locations were found in ACEs than in CEs (Fig. 4-5B,E). These data indicate a similar preferential use of the outer cores and inner periphery of ACEs compared to CEs while drifter data in CEs was approximately double that of ACEs.

\subsubsection{Factors influencing CPUE}

Spatial, temporal and environmental factors were examined for their influence on swordfish catch using GAM analysis. The final model accounted for $46.5 \%$ of the variability in CPUE and included location, month, monthly mean SST, monthly mean SSH, depth of the DSL, 

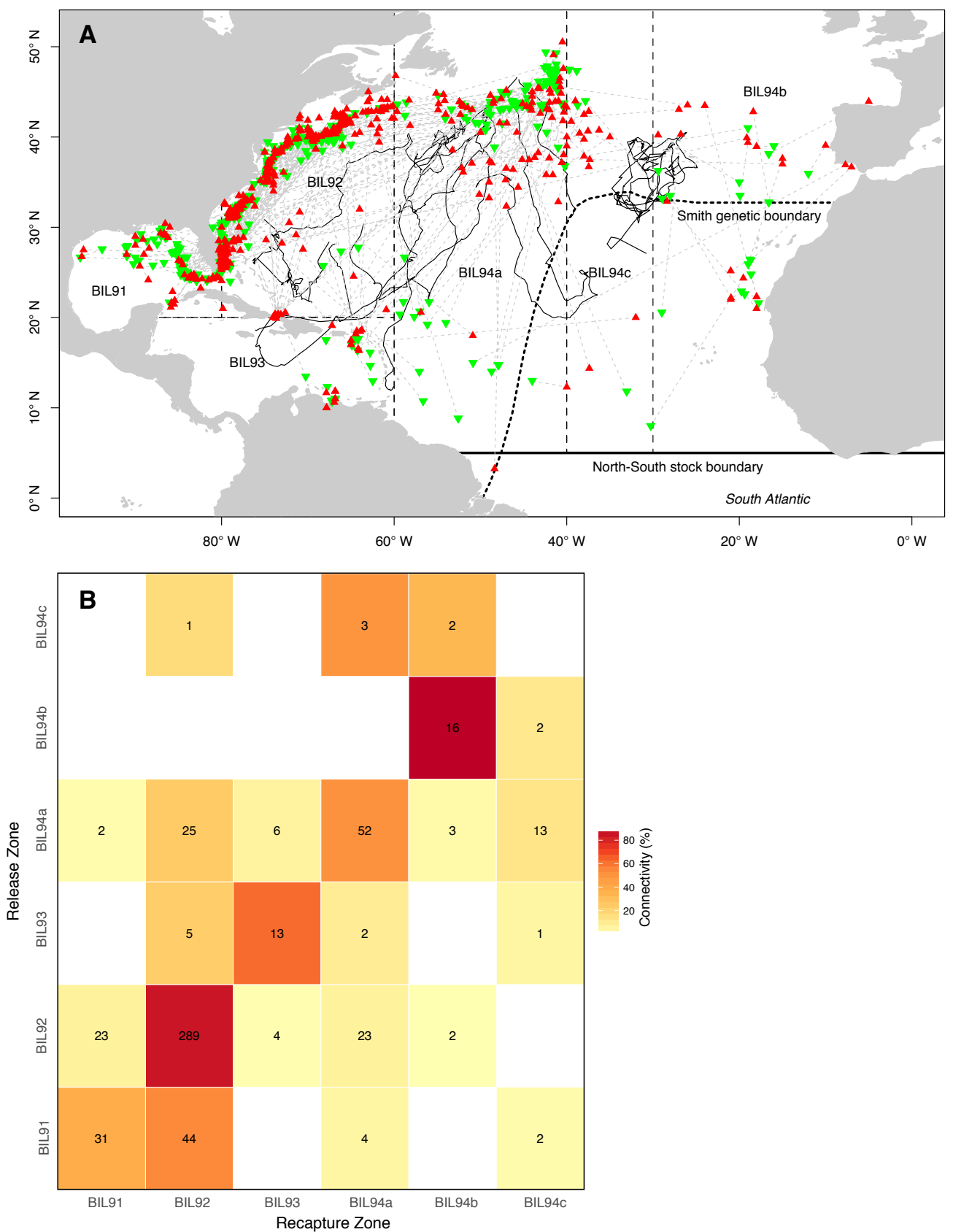

Figure 4-3: Movements (A) of satellite-tagged (black solid lines) and conventional-tagged (dashed grey lines) swordfish in the north Atlantic relative to ICCAT sampling areas (black dashed lines, labeled "BIL9x"). Heavy latitudinal line at $5^{\circ} \mathrm{N}$ indicates north-south stock boundary, and dashed black line from South America to northern Africa is the boundary line from genetic evidence in Smith et al. (2015). Release (green triangle) and recapture (red triangle) positions of conventionaltagged individuals are shown for recovered individuals. Percent connectivity (B) matrix for release and recapture zones of conventional-tagged swordfish is scaled to total number of releases per zone. Numeric labels indicate relevant number of individuals. 
mixed-layer depth, distance to the $200 \mathrm{~m}$ bottom contour and log-transformed chlorophyll as significant predictors (Fig. 4-6). Location was identified as the primary predictor of CPUE (explained $37.9 \%$ of variability), followed by SST (3.1\%) and chlorophyll (2\%). The remaining significant predictors each accounted for less than $2 \%$ of the variability in CPUE: MLD, DSL, SSH, month and distance to the $200 \mathrm{~m}$ bottom contour. Monthly front frequency and SSH range per cell were both insignificant predictors of CPUE, as was distance to the climatological monthly mean of the north wall of the Gulf Stream.

Based on the GAM smoother functions, catch was highest between $17-23^{\circ} \mathrm{C}$ (Fig. 4-6A), in low chlorophyll $\left(<0.15 \mathrm{mg} \cdot \mathrm{m}^{-3}\right)$ environments (Fig. 4-6F) and close to the $200 \mathrm{~m}$ bottom contour (Fig. 4-6E). Catch was also negatively influenced by the most shallow MLDs (< $30 \mathrm{~m}$ )(Fig. 4-6D) and was higher at mid-range scattering layer depths (350-500 m) than outside that range (Fig. 4-6C). The final GAM also found higher catch in areas with high SSH and a similar but weak positive relationship with low SSH (Fig. 4-6B).

\subsection{Discussion}

While over 90 archival tags have been deployed on swordfish in the Atlantic (Braun et al., 2015a), the oceanographic drivers of swordfish movements remain poorly understood. This is at least partially due to a consistent pattern of diel vertical migration by swordfish that often renders traditional light-based geolocation difficult (Dewar et al., 2011; Lerner et al., 2013). Instead, most studies have focused on vertical habitat of swordfish in isolation from the geographic space that the vertical behaviors occurred in due to inadequate light data for reliable geolocation (Abecassis et al., 2012; Dewar et al., 2011; Evans et al., 2014; Loefer et al., 2007; Lerner et al., 2013). Here, we employed a recent advance in geolocation analysis methods to supplement missing light data with other forms of data recorded by the satellite tags (Braun et al., 2018a,b, Chapters 2, 3). Depth-temperature profile data on the tags proved particularly valuable for improving geolocation estimates and enabled a more realistic representation of swordfish movements and the oceanographic environments they inhabit.

\subsubsection{Movements and connectivity}

Swordfish in the western North Atlantic exhibit largely North-South movements that are likely driven by contrasting ocean regimes used for feeding and reproduction (Sedberry and Loefer, 2001; Abascal et al., 2015). Adults moved from productive foraging grounds in the far north during summer to tropical and subtropical waters in winter to presumably spawn in warm waters that promote larval growth (Arocha and Lee, 1995). The timing of this migration varied among individuals, but, in general, adults occupied a relatively restricted area within temperate waters from Cape Cod to the Grand Banks during June to October then moved south to a wider region in the (sub)tropics to the Sargasso Sea, Caribbean 

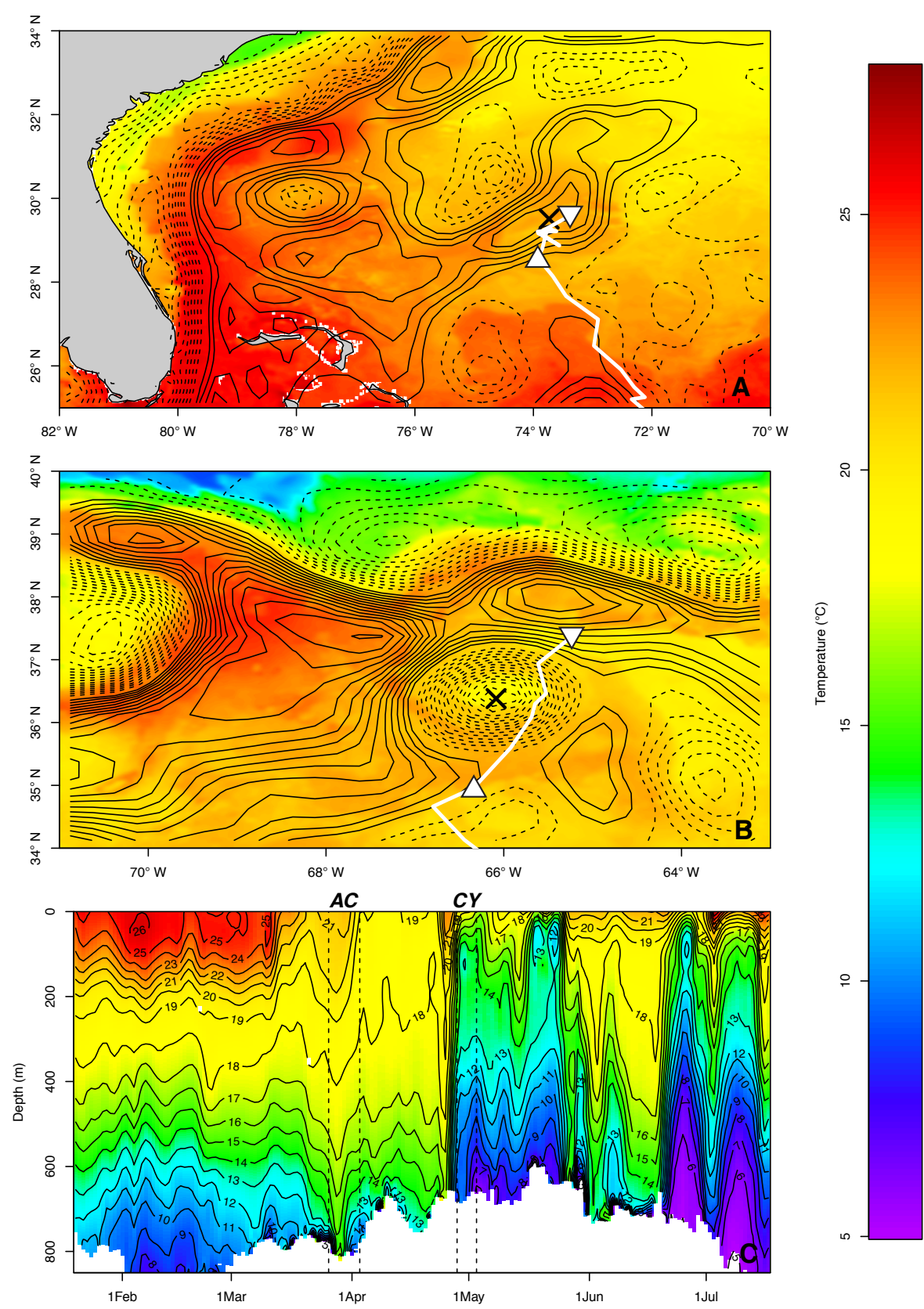

Figure 4-4: Occupation of an open ocean anticyclonic eddy for 9 days (2011-03-26 to 2011-0403) (A) and a cyclonic eddy in the Gulf Stream for 5 days (2011-04-29 to 2011-05-01) (B) by a PSAT-tagged swordfish shown in maps of SST (color; A,B) and SSH (contours; A,B). Contours $(\mathrm{A}, \mathrm{B})$ indicate negative and positive $\mathrm{SSH}$ in dashed and solid lines, respectively, at $5 \mathrm{~cm}$ intervals. Dates of the imagery are shown in the top left of map panels (A,B). Upward and downward facing triangles represent entry and exit of the outer contour $\left(L_{s}\right)$ of the eddy, respectively, and correspond to dashed vertical lines in the profile plot $(\mathrm{C})$. Note that the satellite imagery used in each panel corresponds to the midpoint in time of each eddy occupation. The bounds of the eddy in the imagery may not correspond to the entry and exit points of the eddy denoted by the triangles as the eddies moved considerably over the period in which the swordfish occupied these features. Depthtemperature profiles from the PSAT tag (C) show a vertical cross section of the occupied eddies $(\mathrm{AC}=$ anticylonic, $\mathrm{CY}=$ cyclonic $)$. Contours show isotherms at $1^{\circ} \mathrm{C}$ intervals. 
and Gulf of Mexico from December to March (Neilson et al., 2009). Previous studies have shown a diversity of movements within this general pattern that may be influenced by adult fish homing to spawning habitats (Neilson et al., 2009) while smaller individuals exhibit more variability in movements (Abascal et al., 2015). Results from this study corroborated findings of restricted movements among smaller, immature individuals. The movement data from the Azores was comprised entirely of this younger demographic, and these individuals moved between the Azores Front and the Azores archipelago. This may reflect the dominance of foraging needs among immature individuals that changed with ontogeny to larger-scale movements related to spawning. Indeed, mature individuals in this study moved into known spawning areas of the southern Sargasso Sea and Antillean Arc during the spawning period (December through February) (Neilson et al., 2013). However, individuals were typically tagged months earlier and thousands of kilometers away and were not sexed nor was maturity assessed. As such, we are unable to determine the contribution of reproduction to the observed movements.

A recent review of swordfish population connectivity and stock boundaries (Neilson et al., 2013) suggested that Atlantic swordfish stocks have recovered from historical overfishing impacts, but complex connectivity patterns complicate stock assessments. Genetic evidence supports separation of North Atlantic, South Atlantic and Mediterranean stocks (Bremer et al., 1996; Alvarado Bremer et al., 2005; Smith et al., 2015), and recent work recommended moving the North-South stock boundary as far north as $25^{\circ} \mathrm{N}$ east of $45^{\circ} \mathrm{W}$ (Smith et al., 2015). However, tagging studies (Abascal et al., 2015) suggest a more complex stock structure within the North Atlantic. In our study, no satellite-tagged individuals crossed the current North-South stock boundary at $5^{\circ} \mathrm{N}$, and only one conventional tag deployed near the Grand Banks was recovered across this boundary along the Amazon Delta (Fig. 4-3). In contrast, when considering the proposed boundary based on genetic evidence (Smith et al., 2015), 1 PSAT-tagged individual from the NWA, several from the Azores and 11 conventional tag displacements traversed the $50 \%$ probability contour proposed by Smith et al. (2015) based on single nucleotide polymorphism (SNP) analyses. Thus, most tagging evidence supports amended boundaries for stock designation in the Atlantic, but our study suggests a boundary such as that proposed by Smith et al. (2015) may be less effective than the current designation at $5^{\circ} \mathrm{N}$.

\subsubsection{Oceanographic association}

Ocean dynamics often create barriers to movement, even for highly migratory species, and can have implications from influencing individual behaviors (Stramma et al., 2012) to population-level connectivity (Galarza et al., 2009; Selkoe et al., 2010). Quantitative analy-

sis of oceanographic association by swordfish has largely been restricted to catch data (e.g. Podestá et al., 1993) due to the large error inherent in geolocating PSAT-tagged swordfish. 

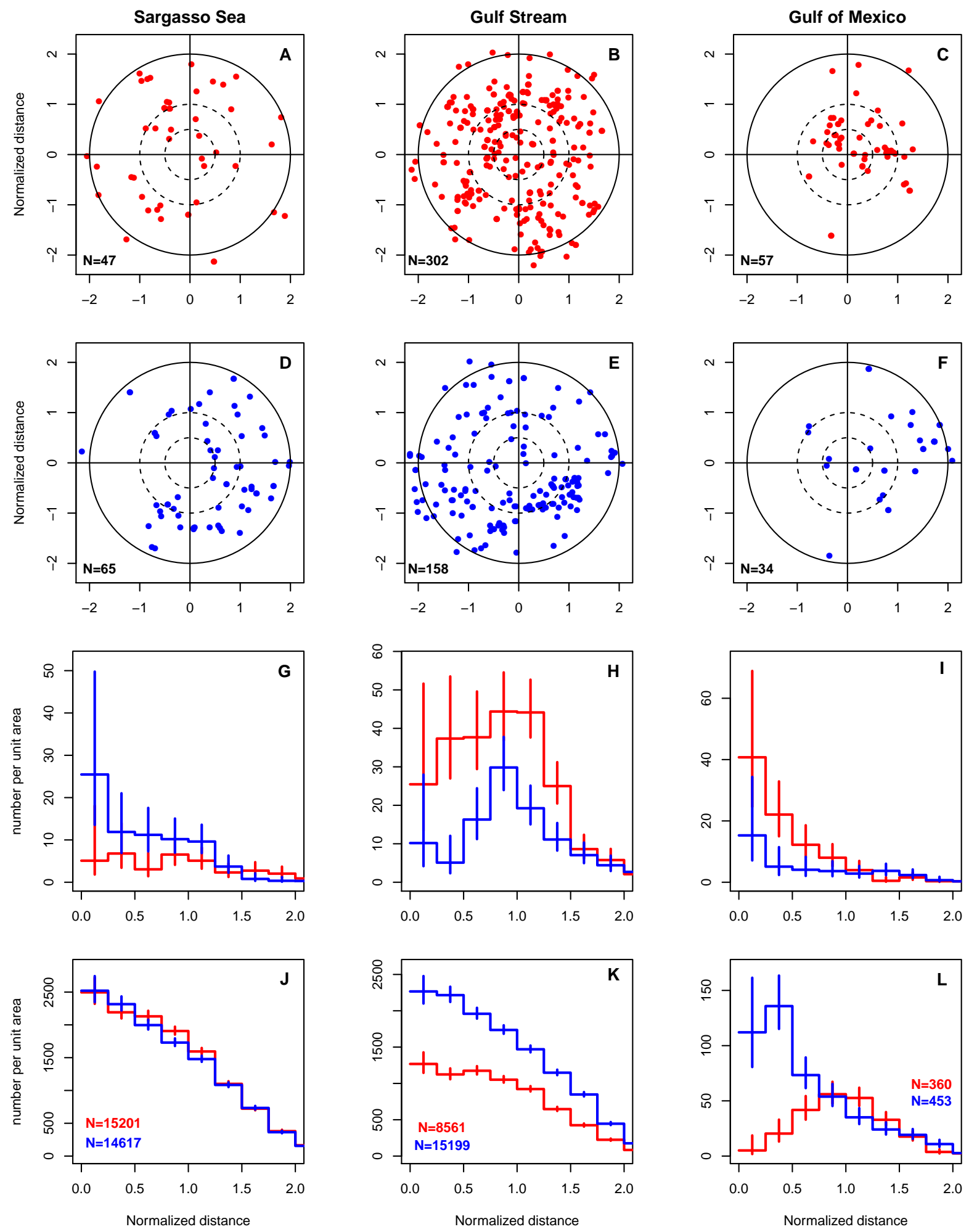

Figure 4-5: Collocation of swordfish conventional tag data to anticyclonic (red) and cyclonic (blue) eddies tracked in maps of remotely-sensed SSH across three dynamic regions of the Atlantic: the Gulf of Mexico, the Gulf Stream and the Sargasso Sea. Tag locations were mapped within an eddy-centric reference frame (anticyclones, A-C; cyclones, D-F). Histograms indicate the mean (and $95 \%$ confidence interval, vertical lines) number-per-unit-area of locations within each portion of the eddy-centric frame for swordfish conventional tags (G-I) and drifters (J-L; see methods). Regions and drifter data are shown in Fig. B-2. 
Those studies that have investigated oceanographic associations have yielded conflicting results, including association to fronts and bathymetric features in some areas (Sedberry and Loefer, 2001) but not in others (Abascal et al., 2010). Here, we improved geolocation estimates from PSAT tag data using depth-temperature profiles and supplemented these results with fisheries data to make robust conclusions about oceanographic association of swordfish in this region.

Several tagging studies have reported extreme physiological versatility exhibited by swordfish that are often observed traversing a wide range of SST (Abecassis et al., 2012). This temperature variability is correlated with other biological, physical and chemical oceano-
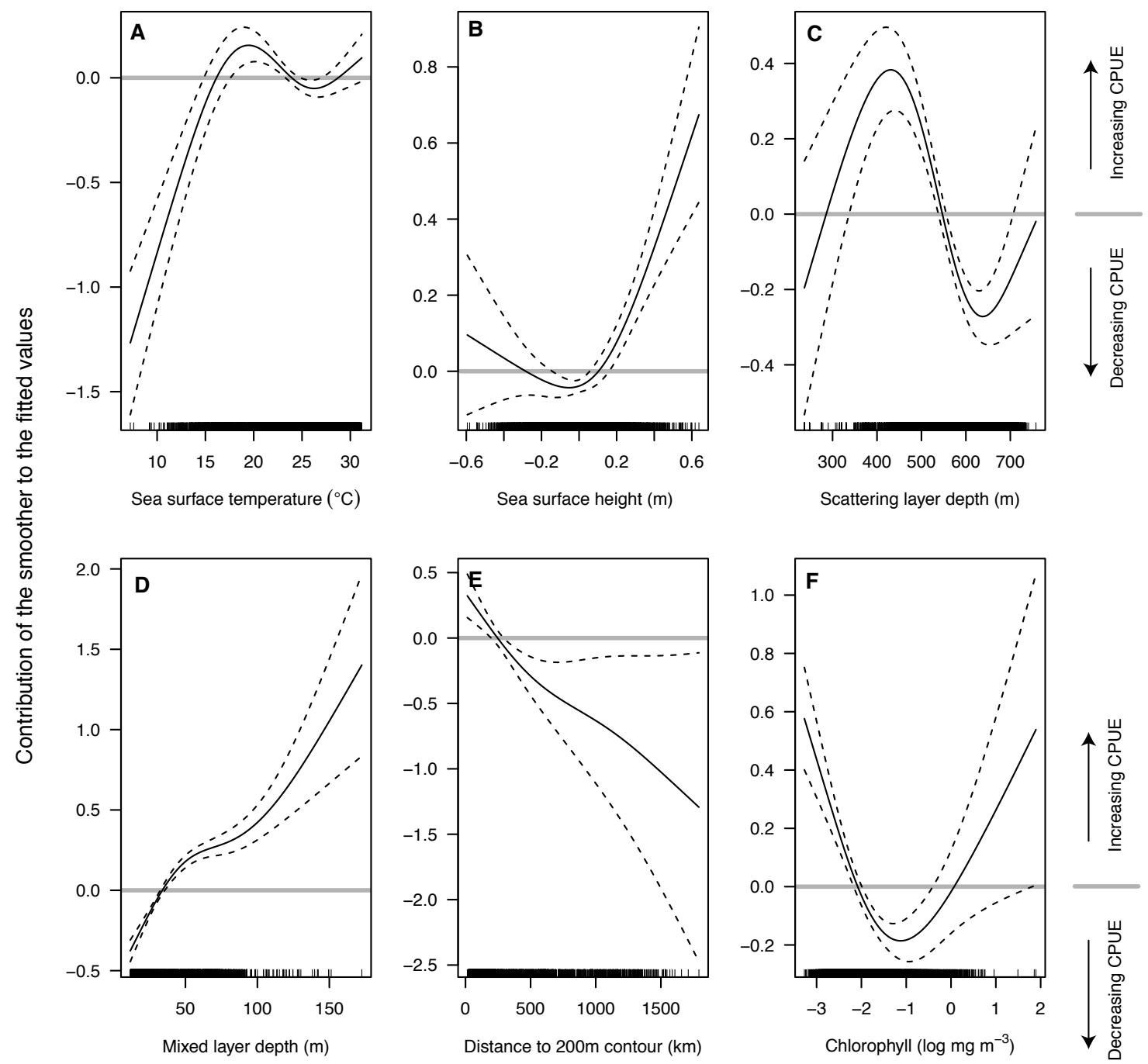

Figure 4-6: Estimated individual effects (solid line) on swordfish catch per unit effort of environmental covariates (A-F). Dashed lines show $95 \%$ confidence limits. Ticks on $\mathrm{x}$-axis indicate values for which data exists. The horizontal line (grey) is added at $\mathrm{y}=0$ to aid visualization. Positive values on y-axis mean higher CPUE. 
graphic characteristics, including chlorophyll concentrations and dissolved oxygen concentrations, that are critical for delineating important habitat for swordfish. For example, Seki Michael et al. (2002) characterized the oceanographic regime on swordfish longline fishing grounds in the subtropical Pacific as highly dynamic (as determined primarily by temperature data) with relatively low chlorophyll $\left(<0.4 \mathrm{mg} \cdot \mathrm{m}^{-3}\right)$. The dynamic nature of swordfish habitat is consistent across ocean basins (Hazin and Erzini, 2008; Seki Michael et al., 2002; Podestá et al., 1993), as is frequent occupation of SSTs $\sim 20-22^{\circ} \mathrm{C}$ (Santos et al., 2006), with reproduction occurring in warmer waters $>22^{\circ} \mathrm{C}$ (Palko et al., 1981; Romeo et al., 2011). Yet many individuals, particularly adults, are regularly observed in temperate waters characterized by cooler SST $<20^{\circ} \mathrm{C}$ (Evans et al., 2014; Abascal et al., 2015), presumably to forage. While DO has been shown to restrict movements in swordfish and other billfish (Stramma et al., 2012; Braun et al., 2015a), it is unlikely to be a major driver of movements in the temperate and subtropical Atlantic. Nearly all PSAT-tagged swordfish in our study transited ambient DO $>3 m L \cdot L^{-1}$, even at depth.

The dynamic oceanographic environments preferred by swordfish are also apparent in their proximity to complex topographic features, such as shelf break habitats, submarine canyons and seamounts, that were frequented by tagged fish (Podestá et al., 1993; Neilson et al., 2009; Palko et al., 1981) and have been shown to positively influence swordfish abundance (Hazin and Erzini, 2008; Bigelow et al., 1999; Scales et al., 2017). Additional inference from our GAM analysis of CPUE data suggested higher abundance of swordfish at intermediate $(350-500 \mathrm{~m})$ scattering layer depths and deeper mixed layers $(>50 \mathrm{~m})$. The correlation between swordfish CPUE and scattering layer depth may be a product of accessibility of DSL organisms to swordfish. Foraging opportunities for swordfish may increase as the surface mixed layer deepens and DSL shallows in a manner consistent with previous studies on scattering organisms in the Atlantic (Marchal et al., 1993). Our MLD results roughly corroborate findings by Scales et al. (2017) in that both found MLD >30 m had a positive influence on swordfish catch. However, Scales et al. (2017) also found a decreasing probability of the presence of swordfish with MLDs deeper than $90 \mathrm{~m}$ based on observer data from the California drift gillnet fishery with spatial and temporal resolution of $0.1^{\circ}$ and 1 day, respectively, that we did not observe in our data. It is possible that the difference in catch rates with depth between the eastern Pacific and NWA are a function of stronger coupling between upwelling in the California Current system and swordfish behavior. However, we would note that there were also significant differences in spatial and temporal resolution between the studies that made direct comparison of results difficult.

\section{Thermal fronts}

Fronts often play a key role in marine ecosystems as they delineate water mass boundaries and can elicit dramatic changes in marine biota (Bakun, 2006). These features are typically 
associated with convergence zones that aggregate particles, including lower trophic level organisms (Olson and Backus, 1985), making them particularly attractive foraging areas for a range of marine species (Bost et al., 2009; Polovina et al., 2000; Scales et al., 2015; Teo et al., 2007; Miller et al., 2015; Queiroz et al., 2016). In our study, association with thermal fronts north of $\sim 25^{\circ} \mathrm{N}$ in the NWA is most likely due to enhanced foraging opportunities during the productive northern summer. Based on stomach contents surveys (Stillwell and Kohler, 1985), cephalopods are the predominant component of swordfish diet during this time period along the shelf break from Cape Hatteras to the Grand Banks, and this region is characterized by complex thermal fronts which have been shown to aggregate one of the swordfish's primary forage species, shortfin squid (Illex illecebrosus)(Wilk et al., 1988). Further south, the use of fronts is typically associated with spawning as these dynamic habitats may aggregate larvae and early juveniles at forage rich frontal zones (Rooker et al., 2012; Suca et al., 2017) and may increase advection toward foraging grounds in the northwest Atlantic by the Gulf Stream (Olson et al., 1994).

In situ SST from the PSAT-tagged individuals in the Azores demonstrated consistent daily SST ranges $>1{ }^{\circ} \mathrm{C}$, suggesting use of front habitats in this region (including the persistent Azores Front). The Azores Front has been shown to effectively capture and aggregate incoming particles (Sala et al., 2016), potentially generating enhanced foraging opportunities (Olson and Backus, 1985) for rapidly-growing juvenile swordfish. If higher forage densities are indeed associated with thermal front boundaries, the regular presence of fronts along, for example, shelf break habitats may provide a predictable feeding location and thus consistent swordfish catch and effort. This seems to be particularly true along the shelf break of the eastern U.S., where previous studies found $80 \%$ of longline fishing effort to be within 40 $\mathrm{km}$ of surface thermal fronts (Podestá et al., 1993), further supporting the conclusions from conventional and satellite tags in this study.

\section{Mesoscale eddies}

Mesoscale eddies have been identified as "hot spots" of biological activity from primary producers (McGillicuddy et al., 2007) to large pelagic fishes (Hobday and Hartog, 2014; Gaube et al., 2018). Some evidence suggests eddies create preferred habitats for many large pelagic predators including yellowfin and bluefin tuna (Teo et al., 2007; Hsu et al., 2015), white sharks (Gaube et al., 2018), marine mammals (Bailleul et al., 2010) and seabirds (Tew Kai et al., 2009). While divergent trends between swords and other predators have been found on NWA feeding grounds in which swordfish were more often found outside of eddies than within them (Hsu et al., 2015), our results corroborate additional findings by Hsu et al. (2015) which suggest that within eddies swordfish prefer ACEs over CEs in the Gulf Stream region, which has also been shown in the California Current (Scales et al., 2017). Conventionally tagged swordfish in our study were caught more often in ACEs than CEs in 
the Gulf Stream region, suggesting a preference for ACEs by the integration of individual swordfish behavior and fishing effort measured by conventional tags. A similar pattern was observed in the Gulf of Mexico despite a significantly reduced sample size. Conventional tag data in the Sargasso Sea suggested higher occupation of cyclones, perhaps due to warm water at depth regardless of eddy polarity and the relatively higher productivity in cyclones (Gaube et al., 2014) in this region (except see Dufois et al., 2016). We cannot tease apart these interacting effects of fish ecology and fisher behavior from our data. However, ACEs in the Gulf Stream region are characterized by high temperature anomalies at depth which may make their deep scattering layer prey more accessible in these environments as thermal constraints may be alleviated (Gaube et al., 2018). In addition, while ACEs are predominantly characterized by low surface chlorophyll (Gaube et al., 2014), they can also exhibit high surface chlorophyll, particularly in subtropical gyres (Dufois et al., 2016), and have been recorded containing anomalously high biomass at depth (Fennell and Rose, 2015). Conventional and satellite-tagged swordfish also occupied CEs, suggesting that their versatile physiology allows them to exploit DSL organisms even in low-temperature environments at depth. The results of the eddy collocation in this study were further supported, at least for ACEs, by the GAM analysis of catch data. This provides additional evidence for swordfish occurrence in high SSH environments (ACEs) across the North Atlantic.

Future investigations of swordfish occupation of eddies, particularly using conventional tag data, should seek to control for the effect of fishing effort on these results. An additional "control" experiment could be added to determine the influence of eddy population in each region. For example, the Loop Current in the Gulf of Mexico only generates anticyclones which may drive significantly different eddy use dynamics by pelagic predators in this region relative to regions such as the Gulf Stream with approximately equivalent generation of eddies of each polarity.

\subsection{Conclusions}

While swordfish stocks have likely recovered from overfishing in the Atlantic basin, several outstanding questions hinder more robust stock assessment and improved understanding of species' response to climate change. We analyzed the results of a fisheries-independent electronic tagging program in the context of fisheries data. Our approach facilitated inference from decades of data-rich, but summarized, fishery records and independent, high-resolution tag data. Together, these datasets suggest limited connectivity between North and South Atlantic stocks, but we also found little exchange between the northeast and northwest Atlantic. Despite these apparent barriers, swordfish are clearly able to traverse dramatically different oceanographic regimes that may help this species adapt to a changing ocean. Swordfish in our study clearly frequented (sub)mesoscale features throughout their range; 
however, improvements in tag technology and associated error will be required to more adequately characterize the relationship and relative importance of fronts and eddies for swordfish in the NWA and beyond. 
THIS PAGE INTENTIONALLY LEFT BLANK 
Chapter 5

\section{WARM CORE RINGS RELEASE PREDATORS FROM THERMAL CONSTRAINTS WHEN FORAGING IN THE OCEAN TWILIGHT ZONE}

This work is a collaborative effort among the follow co-authors: C.D. Braun, P. Gaube, T. SinclairTaylor, G.B. Skomal and S.R. Thorrold.

C.D.B. and S.R.T. designed the study. C.D.B, T.S.T. and G.B.S. conducted the tagging; C.D.B. performed the analysis with contributions from P.G. and S.R.T. C.D.B. wrote the paper with contributions and final approval from all authors. 


\subsection{Abstract}

Mesoscale eddies comprise the "internal weather" of the ocean and can generate first order perturbations of open ocean ecosystems (Mahadevan, 2014; McGillicuddy et al., 2007). The Gulf Stream generates some of the most energetic eddies and meanders found anywhere in the global ocean. Counterclockwise-rotating cyclonic eddies trap cold, nutrient-replete water from north of the Gulf Stream during formation and clockwise-rotating anticyclonic eddies characterized by anomalously warm water that is low in chlorophyll and nutrients due to its source in the northern Sargasso Sea (Gaube and McGillicuddy, 2017). Using new observations from pelagic predators and a constellation of earth-observing satellites, our results challenge the existing paradigm that warm core rings are generally unproductive (Gaube and McGillicuddy, 2017; Williams and Follows, 1998). Here, we show that blue sharks (Prionace glauca), a model epipelagic predator, actively seek out the interiors of anticyclonic Gulf Stream eddies where they most often exhibit behaviors indicative of foraging. Using > 2,000 tracking days and nearly 500,000 high-resolution time series measurements collected by 15 instrumented blue sharks, we show that these "evolutionarily-informed oceanographers" regularly dive deep into the mesopelagic $(>200 \mathrm{~m}$ ) in anticyclones where anomalously warm temperatures alleviate a physiological constraint that otherwise isolates mesopelagic fish biomass from many epipelagic predators. Anticyclonic eddies thus provide a conduit by which surface-oriented predators access the most abundant fish community on the planet (Irigoien et al., 2014). The results presented here provide valuable new insight into open ocean habitat use by large pelagic predators that should be incorporated into dynamic ocean management approaches. Furthermore, our results shed new light onto the ecosystem value of mesopelagic prey, suggesting additional considerations are necessary before planned biomass extraction from the Ocean's twilight zone as these activities could interrupt a key link between planktonic production and top predators (Smith et al., 2011). 


\subsection{Main Text}

The pelagic ocean represents the largest habitat on Earth (99\% of the biosphere Game et al., 2009) and yields $>80 \%$ of the fish consumed by humans (Game et al., 2009; Pauly et al., 2002). Mesoscale eddies are ubiquitous, energetic features that structure open ocean ecosystems (McGillicuddy, 2016) on time scales of weeks to months and spatial scales $\mathcal{O}(10 \mathrm{~s}-100 \mathrm{~s}$ $\mathrm{km})$. These features are known to control biogeochemical fluxes and transport entire pelagic communities (Chelton et al., 2011). While eddies are known to affect biological communities from plankton (McGillicuddy et al., 2007; Benitez-Nelson et al., 2007) to predators (Bailleul et al., 2010; Gaube et al., 2018), studies of the coupling of biology and ocean physics at the oceanic mesoscale remain inconclusive with respect to the role of anticyclones in maintaining preferential foraging conditions for upper trophic levels. Fish communities, in particular, are difficult to quantitatively link to mesoscale oceanographic features due to constraints imposed by traditional light-level geolocation (Braun et al., 2018a, Chapter 2). Yet, if fishes are able to sense favorable conditions associated with mesoscale eddies as has been shown for turtles (Gaube et al., 2017; Kobayashi et al., 2011), seabirds (Tew Kai et al., 2009), mammals (Bailleul et al., 2010), and indeed large white sharks (Gaube et al., 2018), eddies may have profound impacts on many commercially- and ecologically-important fish species.

Recent advances in satellite oceanography have facilitated the automatic identification and tracking of mesoscale eddies. Cyclonic eddies (CEs) in this region (including cold-core rings generated by the Gulf Stream) typically trap cold, productive water from the New England shelf slope during formation (Pingree et al., 1979; Group, 1981) while anticyclones (ACEs, or warm-core rings) transport anomalously warm, low productivity Sargasso Sea water north of the Gulf Stream (Joyce, 1985; Franks et al., 1986). Due to these characteristics, ACEs have been characterized as warm, ocean "deserts" with low productivity (Williams and Follows, 1998), particularly in the Gulf Stream (Gaube et al., 2014) (except see Franks et al., 1986; Nelson et al., 1989). Recent work on predators in this region suggests that white sharks (Gaube et al., 2018) and swordfish (Chapter 4) may preferentially occupy anticyclones, but inference in these studies was limited by sample size and fisheriesdependence, respectively. Here we report high-resolution, three-dimensional movements of 15 blue sharks in the Gulf Stream eddy field. The results document preferred occupation of the cores of anticyclonic eddies, particularly while sharks exhibited horizontal movements indicative of foraging, that are counter to the paradigm of low biomass in open ocean anticyclones. Diving behavior in these features demonstrated the thermal constraints operating on an ectothermic, seemingly epipelagic predator and highlight unrecognized connections between epipelagic predators and mesopelagic prey that are modulated by ocean dynamics.

We tagged 15 blue sharks each with 2 types of satellite tags: a satellite-linked position transmitter (SPOT tag) mounted on the dorsal fin and a pop-up satellite archival trans- 

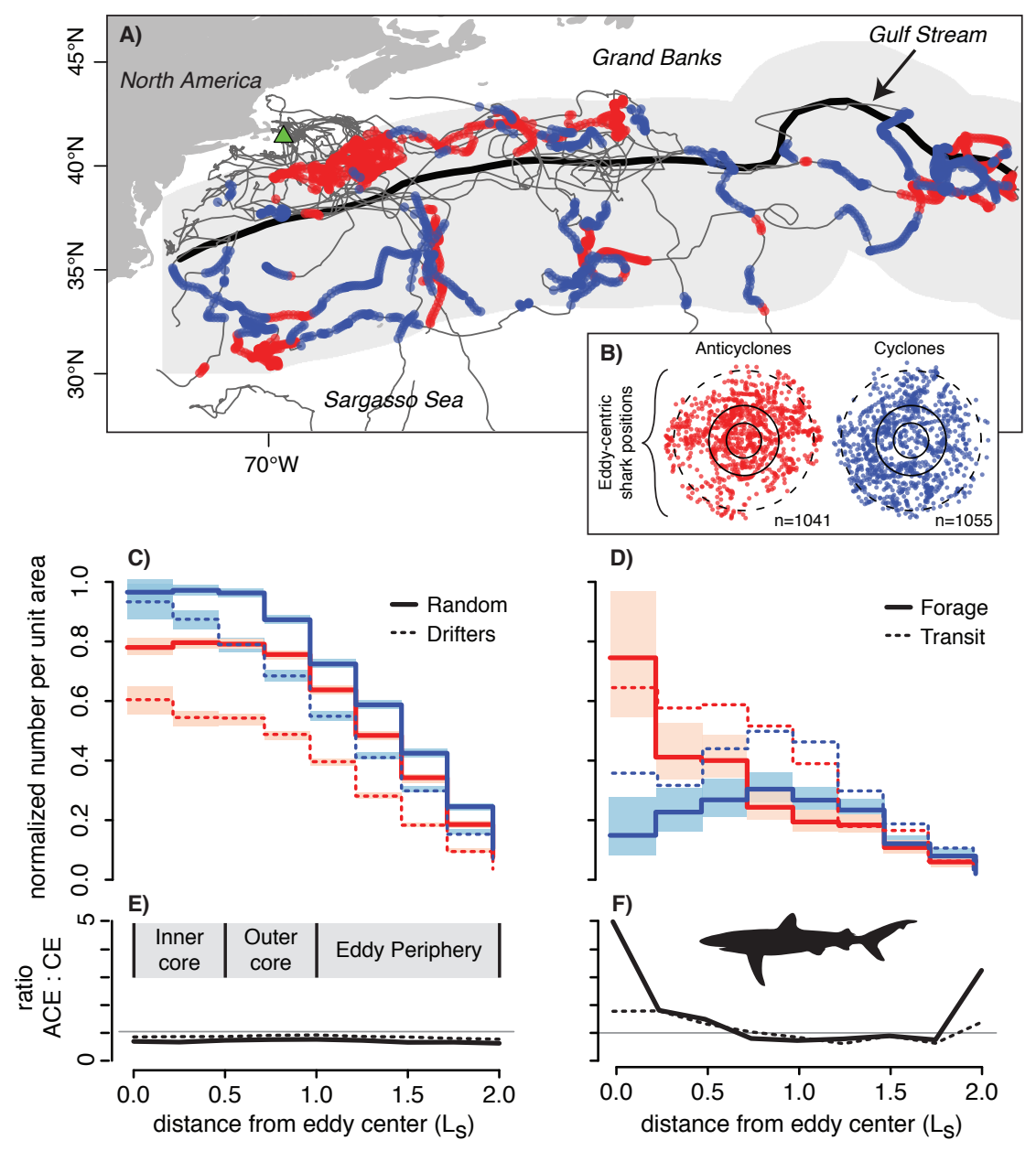

D)
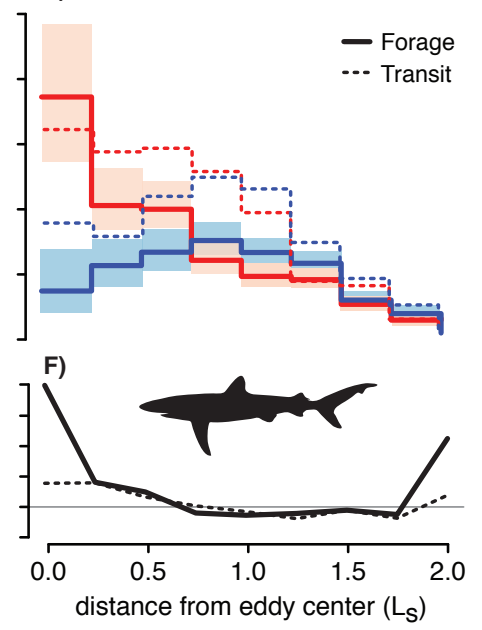

Figure 5-1: Blue sharks tagged in New England frequented the Gulf Stream eddy field (A) and occupied anticyclones (red throughout) and cyclones (blue throughout) at approximately the same frequency (B). Eddy-centric histograms indicated random walk simulations (solid, C) and passive drifters (dashed, C) exhibited higher cyclone use after controlling for eddy area. Sharks (D) used eddy peripheries $\left(>L_{s}\right)$ approximately equally between eddies of either polarity, but more positions classified as "transiting" (dashed, D) were collocated around the eddy length scale $\left(L_{s}\right)$ compared to "foraging" locations (solid, D). Sharks showed a marked preference for the cores of anticyclones relative to cyclones, particularly while foraging (D). The ratio of anticyclone (ACE) to cyclone (CE) positions across different regions of the eddies are shown for random walk simulations (E), drifters (E) and foraging (solid) and transiting (dashed) modes in the shark data (F). Note confidence intervals have been removed from the transit mode in panel D to aid visualization. 
mitting (PSAT) tag that recorded high-resolution depth and temperature time series. Two additional sharks were tagged with SPOT tags only. All 17 SPOT tags reported a total of 8,279 positions over 2,117 tracking days (mean 4 positions $\cdot$ day ${ }^{-1}$; deployment duration range 54-288 days). A hierarchical state-space movement model was used to delineate behaviors in the movement data indicative of "foraging" (area-restricted search) and "transiting" based on correlation and turn angles (Jonsen, 2016). Movements were combined with $\sim 482,000$ depth and temperature time series data points at 2.5 minute resolution from 12 PSAT tags at liberty for an average 129 days (range 16-180) to reconstruct blue shark movements in 3D. On average, overall movements covered 5,454 km (range 2,280-14,485 km) in up to 288 days at liberty and were predominantly oriented east-west at temperate latitudes (Fig. 5-1A). PSAT-tagged sharks made extensive vertical movements offshore where they regularly dove to at least $400 \mathrm{~m}$ (maximum depth 1,696 m), almost exclusively during daylight hours (Fig. 5-2). Sharks occupied a $26^{\circ} \mathrm{C}$ temperature range (mean $18.5^{\circ} \mathrm{C}$, range 4.0-29. $6^{\circ} \mathrm{C}$ ) but remained above the $12^{\circ} \mathrm{C}$ isotherm while diving (97\% of temperature data $>12^{\circ} \mathrm{C}$; Figs. 5-2, 5-3), suggesting thermal constraints prevented use of colder water (Carey and Scharold, 1990).

Blue shark movements and depth-temperature time series data were collocated to mesoscale eddies in the Gulf Stream tracked in maps of sea level anomaly. Area-normalized histograms of shark positions as a function of distance from eddy centers revealed that blue sharks were significantly more likely to be associated with the inner-cores $\left(r \leq L_{s} / 2\right)$ of ACEs than CEs (Fig. 5-1). The preference for ACEs was further strengthened when considering only the foraging behavior mode (Fig. 5-1D). Depth-temperature time series data indicated a total of 15,189 depth measurements were made within eddies representing 452 and 181 cumulative hours within anticyclonic and cyclonic eddy cores $\left(r \leq L_{s}\right)$, respectively. Distribution of time-at-depth in eddies was indicative of blue sharks foraging on diel vertically-migrating, mesopelagic prey during dives as sharks spent $15 \%$ of time in ACEs below $300 \mathrm{~m}$ compared to $1.5 \%$ in Gulf Stream CEs during day and $<1 \%$ in eddies of either polarity during night (Fig. 5-2E,F). While diving, tagged individuals encountered strong positive (warm) temperature anomalies at depth in ACEs (Figs. 5-2, C-1, C-2), including as high as $+10^{\circ} \mathrm{C}$ from 250-350 m (Fig. C-1). Blue sharks experienced significant negative temperature anomalies at depth in some cyclones as predicted by vertical eddy composites (Figs. 5-2, C-1, C-2); however, the majority of diving in CEs occurred in water warmer than that suggested by eddy vertical composites (Fig. 5-2B) or known upward vertical displacement of isotherms typical of Gulf Stream CEs (cold-core rings; Gaube et al., 2014, 2018). Tracking the warm eddies back to their formation indicated these warm cyclonic eddies, not rings, were of Sargasso Sea origin and had moved into the southern portion of the Gulf Stream study area. Further analysis confirmed sharks occupied very few traditional cold-core rings generated by the Gulf Stream (Fig. 5-3E,F) in favor of warm Sargasso-derived CEs instead. 

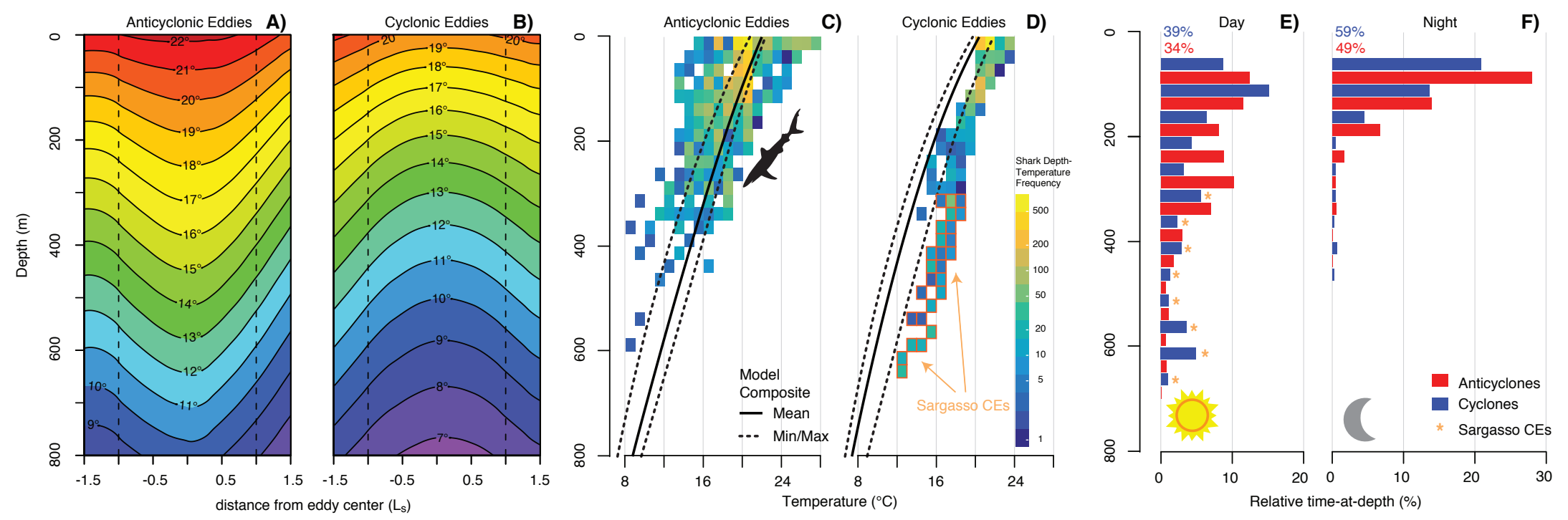

Figure 5-2: Eddy vertical structure and blue shark diving. Modeled depth-temperature profile composites for 27 anticyclonic (A) and 28 cyclonic (B) eddies occupied by blue sharks. Histogram of blue shark depth-temperature data while diving in cores $\left(r<L_{s}\right)$ of anticyclones $(\mathrm{C} ; \mathrm{n}=7,271)$ and cyclones $(\mathrm{D} ; \mathrm{n}=2,521)$ compared to model composite depth-temperature profiles. Summary of blue shark time-at-depth during day $(\mathrm{E})$ and night (F) occupation of anticylonic (red) and cyclonic (blue) eddies. The 0-50m depth bin has been removed to aid visualization. The highlighted depth-temperature cells (orange outline, D) and time-at-depth bins (orange asterisks, E) correspond to diving in cyclones of Sargasso Sea origin. 
The observed differences in temperature at depth among ACEs, Gulf Stream CEs and Sargasso-derived CEs regulated the blue shark diving in these eddies (Fig. 5-3A-C), primarily by modulating the depth of the $12^{\circ} \mathrm{C}$ isotherm (Fig. $5-3 \mathrm{E}, \mathrm{F}$ ). We developed a metric ( $D^{\prime}$; Eq. C.2) to quantify the relationship between shark maximum dive depth relative to the climatological mean depth of the $12^{\circ} \mathrm{C}$ isotherm in which $D^{\prime}>0$ is indicative of anomalously deep diving relative to the climatological mean. This analysis found diving in ACEs was primarily characterized by $D^{\prime}>0$ while CEs more often exhibited $D^{\prime}<0$ (Fig. 5-3E), suggesting eddy modulation of water column structure controlled shark behavior in these features. This was confirmed by comparing climatological mean depth of the $12^{\circ} \mathrm{C}$ isotherm to modeled in situ depth in eddies (Fig. 5-3F).

Our results suggest that blue sharks synthesize an evolutionary "knowledge" of high biomass in the mesopelagic with the constraints of their thermal physiology by using warm, anticyclonic eddies as conduits to the deep ocean. This contrasts with some previous findings that suggest low productivity of anticyclonic eddies but corroborates recent observations that, in contrast to their low chlorophyll surface signature, ACEs can contain among the highest deep scattering layer densities in the world oceans (Fennell and Rose, 2015). In addition, other recent work has shown that some specific types of ACEs contain enhanced primary productivity in their cores (Dufois et al., 2016). While we are unable to attribute specific behaviors to $P$. glauca during dives, previous studies tracking blue sharks in the Gulf Stream reported a high frequency of octopods, a diel vertical migrator that occurs principally below $250 \mathrm{~m}$, in blue shark stomachs (Carey and Scharold, 1990). In addition, mesopelagic dives by blue sharks were nearly always during daytime when the bulk of the mesopelagic community is at depth (Bianchi et al., 2013), and shark dive profiles were characterized by rapid descents with slower ascents, a pattern interpreted as prey searching behavior in sharks and tunas (Carey and Scharold, 1990; Brunnschweiler et al., 2009). Taken together, deep dives suggest blue sharks are foraging for cephalopods and mesopelagic fishes, some of the blue sharks primary prey items (Nakano and Stevens, 2008), that are known to concentrate at depth in the Gulf Stream (Fedulov and Arkhipkin, 1986) and have been collected in high densities in ACEs (Fennell and Rose, 2015; Godø et al., 2012). However, additional research will be needed to confirm this hypothesis.

While the mesopelagic contains the highest fish biomass on earth (Irigoien et al., 2014), the deep ocean presents significant physiological challenges for epipelagic predators. ACEs may provide a unique conduit from the surface to deep ocean due to anomalously warm temperatures at depth where high acoustic scattering (a proxy for prey availability or biomass) at depth has been shown to have a strong positive correlation with temperature (Fennell and Rose, 2015). While Gulf Stream cyclones can contain significant productivity (Gaube and McGillicuddy, 2017), our data suggest the thermal constraints to diving in these features disconnect the epi- and mesopelagic fish communities. Indeed, recent work has suggested 

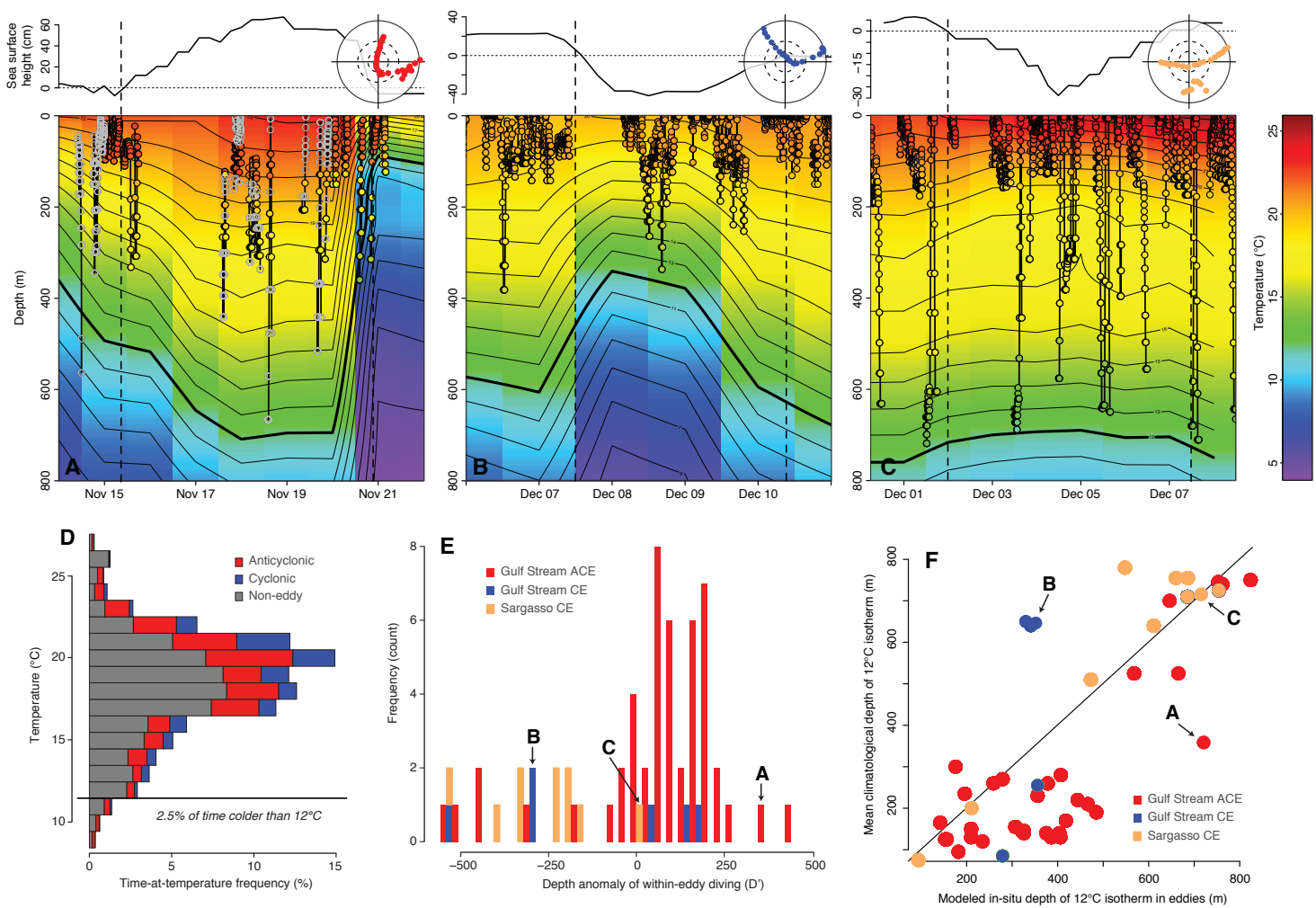

Figure 5-3: Example diving by blue sharks in anticyclonic (A) and cyclonic (B) Gulf Stream eddies and a cyclonic eddy of Sargasso Sea origin (C). Upper line plots indicate measured sea surface height from AVISO (Eqn. C.1). Top right circular plots in each panel show geographic movements of the shark in eddy-centric coordinates (as in Fig. 5-1). Water column structure in the eddies (color map, top panels) is modeled depth-temperature data from HYCOM. Colored points connected by solid vertical lines represent shark diving behavior (grey circles indicate no temperature data was acquired/transmitted by the tag for a given depth point in the time series). Dashed vertical lines indicate entry and exit of eddies based on SSH. Thin, labeled horizontal lines indicate isotherms at $1{ }^{\circ} \mathrm{C}$ intervals. Frequency histogram of the time-at-temperature distribution for all shark dive data in the Gulf Stream (D) and for the depth anomaly of within-eddy diving (E; see Eq. C.2). Positive $D^{\prime}$ indicates sharks dove below the climatological $12^{\circ} \mathrm{C}$ isotherm, suggesting vertical downward displacement of isotherms by the eddy as shown by comparing climatological mean isotherm depth to in situ modeled isotherm depth within eddies (F). 
endothermic white sharks are likely able to forage deep in Gulf Stream cyclones but that dive times are shorter than in ACEs (Gaube et al., 2018). Previous studies have suggested the thermoregulatory implications of deep diving for several epipelagic predators, including elasmobranchs (Thorrold et al., 2014), marine mammals (Tyack et al., 2006) and commerciallyimportant fishes (Carey, 1982). Work on blue sharks suggests thermal hysteresis facilitates maintenance of body temperatures significantly above ambient in the mesopelagic followed by abrupt termination of deep dives when muscle cools to $<15^{\circ} \mathrm{C}$ (Carey and Scharold, 1990). We observed similar oscillatory diving into the mesopelagic during daytime in both types of Gulf Stream eddies that was seemingly constrained by temperature at depth (Fig. 5-3), consistent with the behavioral thermoregulation observed by Carey and Scharold (1990). Sharks spent more time deeper in ACEs (Fig. 5-2) than Gulf Stream CEs, regularly as deep as 300$350 \mathrm{~m}$, where backscatter is reportedly high (Fennell and Rose, 2015) and temperatures were consistently $>12^{\circ} \mathrm{C}$. Thus, it seems the potentially enhanced foraging opportunities combined with relaxing of thermal constraints in ACEs may interact to drive blue shark preference for these features.

Our results highlight the potential role of oceanography in modulating the connectivity between epipelagic predator communities and the twilight zone. This region has proven particularly difficult to study but likely supports a number of commercially-important fishes (such as sharks, swordfish and tunas) and may provide significant support for open ocean foodwebs. We show that dynamic mesoscale eddies modulate this connection, yet current management approaches largely ignore the highly dynamic ocean in favor of traditional, static management approaches (Maxwell et al., 2015). Finally, we provide additional evidence of the value of mesopelagic communities to pelagic predators, suggesting extraction of deep ocean resources may interrupt this key resource.

\section{METHODS SUMMARY}

Detailed methods and supporting figures and tables can be found in Appendix C.

\section{Satellite tagging and track analysis}

Blue sharks were tagged with electronic tags that provided both accurate $(<10 \mathrm{~km}$ error $)$ satellite-based positions and a time series of depth and temperature every 2.5 minutes. Locations were filtered to remove spurious positions and data gaps $>4$ days. Filtered tracks were fit in a hierarchical fashion with a two-state switching state-space model to standardize the location time series and infer behavior state (Jonsen, 2016). Resulting positions were used to collocate depth and temperature data for reconstruction of 3D movements. 


\section{Eddies}

Eddies were identified and tracked in daily maps of surface altimetry. A custom meander filter was used to remove Gulf Stream meanders before shark movement data was collocated to the nearest identified eddy in the atlas. Eddy subregions were defined according to the radial distance from the eddy center (Gaube et al., 2017). Null eddy use was quantified by two independent methods: shark movements simulated by random walks and 5 years of surface drifter data. Eddy vertical composites were constructed for shark-occupied eddies using HYCOM modeled depth-temperature profiles and anomalies used climatological mean temperature from the World Ocean Atlas. 
Chapter 6

Conclusion AND OUtLoOK 


\subsection{Summary}

Historically, observing movements of marine fishes has been limited by error in geolocation. These complications have rendered contextualizing observed behaviors and subsequent inference difficult. Consequently, oceanographic associations of many commercially-important or conservation-relevant species remains poorly understood; yet, these animals are immersed in and constantly interacting with a dynamic ocean. In this thesis, I developed a model framework that leverages 3D oceanographic data to improve geolocation error and demonstrated its utility for two species that, historically, have proven challenging to geolocate. This method is poised to leverage future advances as oceanographic data and models continue to improve.

Using the approach I developed and validated in Chapter 2, I applied the model in Chapter 3 to study basking sharks in the North Atlantic using a high-resolution oceanographic model. Sharks in this study were tagged around Cape Cod, MA and moved as far as SE Brazil, a total distance of $>17,000 \mathrm{~km}$, and $59 \%$ of tracked individuals exhibited seasonal fidelity to the northeastern US shelf. During these movements, basking sharks made impressive vertical movements to $>1,500 \mathrm{~m}$ and regularly occupied the mesopelagic, often for months at a time. This study draws attention to the poorly understood ecology of basking sharks and demonstrates the need for international cooperation in managing this highly migratory species that traverses the jurisdictions of dozens of countries each year.

I also applied the model framework from Chapter 2 to study the broadbill swordfish (Chapter 4). Due to diel vertical migration and often aphotic behavior, the swordfish has historically proven difficult to study with traditional light-based geolocation. By leveraging significant improvements in geolocation error, I was able to describe mesoscale eddy use by some satellite-tagged individuals in this study. I validated the tag-based observations of eddy use by integrating 2 large fisheries datasets with the independent satellite tag data. Overall, I found swordfish exhibited extreme physiological versatility by occupying a $25^{\circ} \mathrm{C}$ SST range; however, satellite-tagging and fisheries data independently suggested swordfish preferred a much narrower SST range in oligotrophic waters with active front boundaries. Overall, this study makes significant contributions to swordfish ecology and fisheries oceanography by quantifying some of the critical oceanographic influences on swordfish.

In the final data chapter (Chapter 5), I used the "lessons learned" from the limitations of the previous chapters to design a tagging study to specifically quantify oceanographic associations of blue sharks, as a model pelagic predator. I focused this analysis on the eddy-rich region of the Gulf Stream where I was able to observe blue shark movements and behavior in high-resolution, 3-D as they moved through the eddy field. Using this data, I discovered that blue sharks actively sought the interiors of anticyclonic Gulf Stream eddies. Some have characterized warm core rings as low productivity and anomalously warm, 
"desert-like" environments. Yet, blue sharks exhibited behaviors indicative of foraging in these features. With high-resolution dive data, I found blue sharks were regularly diving into the mesopelagic during the day in anticyclones, suggesting warm water at depth in these features may alleviate thermal constraints to diving into the mesopelagic to forage. This study provides important insight into the connectivity between epi- and mesopelagic ecosystems and suggests mesopelagic fishes may be a key link between planktonic production and top predators.

\subsection{Outlook}

As with any scientific endeavor, many assumptions were necessary in each of these studies, and all left me with more fascinating questions to pursue. The modelling approach developed in Chapter 2 was an effective step toward building a flexible, transferable framework that can be readily adapted to animal movement problems in a wide range of taxa and environments. However, many improvements could be implemented. The primary limitations of HMMoce in its original form were its reliance on deprecated, manufacturer-specific software for lightbased position estimates as well as support for tags built by a single manufacturer. This was appropriate for our needs, but it was apparent that additional compatibility would be required for widespread adoption of these methods. Further development to incorporate some of these changes are currently underway. In addition, additional process models (e.g. including advection) and algorithm support (e.g. Viterbi) will dramatically improve utility of this model for the broader community.

While the approach in Chapter 3 demonstrated remarkable improvements over previous studies to geolocate a traditionally challenging species, the resulting study was a primarily qualitative depiction of basking shark ecology in the NWA. Future studies could use this or similar datasets to better quantify oceanographic associations, particularly using the highresolution dive data collected from many of these deployments. In the swordfish analysis in Chapter 4, I was able to quantify habitat use and relate this to oceanography; however, this work would also benefit from further exploration of fine-scale behaviors using the depthtemperature time series from these tags.

Finally, Chapter 5 was my attempt to leverage "lessons learned" from the previous chapters to design, fund and conduct a study from end-to-end that would not be constrained by the analytical limitations I faced in previous chapters. While I believe this was largely successful, the high individual variability in eddy use suggests a potential difference in availability of eddies that may influence blue shark-eddy interaction. Thus, availability of eddies needs to be accounted for in order to quantify relative use of these features. This study would also benefit from incorporating the role of underlying currents in movement patterns, as blue sharks are known to be heavily influenced by advection (e.g. Carey and Scharold, 
1990) and from quantifying fine-scale response to gradients indicative of eddy encounter (e.g. sea surface height). Furthermore, while the results in Chapter 5 suggest blue sharks preferentially occupy and forage in warm core rings of the Gulf Stream, the tag data does not help us differentiate specific drivers of this behavior. For example, is the observed pattern simply a product of higher food availability in warm core vs. cold core rings? Blue shark diet studies suggest primary offshore prey items include cephalopods and opportunisitc foraging on teleosts, including mesopelagic fishes such as myctophids and Alepisaurids (lancetfishes) (Kohler, 1988). Blue shark overwintering in the Gulf Stream (Chapter 5, Campana et al., 2011) qualitatively matches the limited work on cephalopod distribution and abundance in this region. Dawe and Beck (1985) suggested juvenile, potentially more vulnerable, shortfinned squid were concentrated along the north wall of the Gulf Stream and in Slope water. Olson and Backus (1985) and Backus and Craddock (1982) were among the first to sample mesopelagic fish communities of warm core and cold core rings, respectively, but little work has been done since to facilitate direct comparison of the fish or cephalopod communities that characterize mesoscale features in the Gulf Stream. Most of the sampling efforts have described small-bodied cephalopods and mesopelagic fishes from the Gulf Stream region that may have limited ability to move horizontally relative to Gulf Stream flow. Thus, Olson and Backus (1985) provide a potential mechanism for concentrating these potential prey items at depth in warm core rings via entrainment and convergence. Overall, the mesopelagic communities of the Gulf Stream and the influence of mesoscale dynamics (e.g. ring effects on water column structure) on the depth, occurrence and density of these communities remains poorly understood. These, and other analyses, may aid in further interpretation of the mechanisms driving response of pelagic fishes to mesoscale eddies and their potential functional role(s).

Overall, this thesis demonstrates fish clearly use and are influenced by mesoscale oceanographic features in the North Atlantic Ocean. And, in cases like the blue sharks in Chapter 5, some of the impacts of these interactions between biology and ocean physics can span entire communities and marine foodwebs. Fine-scale habitat associations of species not associated with the surface-air interface will likely require a transformative technology to better geolocate these species at depth. For the many species that regularly frequent the surface-air interface, existing Argos and GPS technologies, coupled with high-resolution oceanographic models and growing remotely-sensed datasets, are poised to address the mechanistic drivers of oceanographic feature use by fishes. The main limitation to these approaches will be direct comparison to 3D ocean dynamics (including physics, chemistry and biology) that cannot be characterized with remote sensing. Comparing oceanographic associations of multiple fish species with differing physiology (e.g. endothermic and ectothermic sharks) may help distinguish some of the most important physical-biological interactions in the absence of quality data on 3D dynamics such as prey availability. 


\section{APPENDIX A}

\section{Chapter 2 Supplemental Information}

This chapter was originally published as Braun, C.D., Galuardi, B., and Thorrold S.R. (2018). HMMoce: An $\mathrm{R}$ package for improved geolocation of archival-tagged fishes using a hidden Markov method. Methods in Ecology and Evolution 9, 1212-1220.

C.D.B and B.G. conceived the project and developed the package; C.D.B and S.R.T. collected the data; C.D.B. wrote the paper; B.G. and S.R.T. contributed to the writing of the paper. 


\section{A.1 Supplemental Methods}

\section{A.1.1 The Approach}

We present a state-space model to estimate movements and behavior from uncertain and temporally correlated oceanographic data collected with electronic tags. Estimation of unknown states (movements and behavior) based on observations is formulated as a hidden Markov model (HMM) on a spatial grid in continuous time. While many details set HMMoce apart from other HMM methods for electronic tag data, the logic of the underlying approach is similar and discussed in detail elsewhere (Pedersen et al., 2008, 2011a; Thygesen et al., 2009). A key feature of HMMoce and its predecessors is the discretization of space over a grid. In this manner, location estimation can be supplemented by, or based entirely on, environmental data (e.g. sea surface temperature or oceanographic profiles).

The logic and use of HMMoce is as follows:

1. prepare tag data and acquire necessary environmental data

2. calculate gridded likelihoods based on observations

3. form the model

4. get movement parameters

5. finalize track and space use metrics

\section{A.1.2 Data preprocessing}

The HMMoce package contains several custom routines for reading and formatting Wildlife Computers tag data that can then be leveraged in the observation portion of the model (refer to package vignette for data requirements). Currently, light-based position estimates (GPE2), with manual filtering of clearly spurious positions, are used to define the temporal and spatial limits of interest; but this may be overridden by the user. After tag data preparation, gridded environmental data (e.g. sea surface temperature, SST) is acquired from various online repositories. Gridded monthly climatological mean depth-temperature data from the World Ocean Atlas 2013 (WOA, 0.25 resolution), daily reanalysis model depth-temperature products from the HYbrid Coordinate Ocean Model (HYCOM, 0.08 resolution) and daily optimally interpolated sea surface temperature fields (OI-SST, $0.25^{\circ}$ resolution) are acquired using get.env and the appropriate type argument. See HMMoce package documentation (?get.env) for more details on environmental data and for options to use additional input datasets.

\section{A.1.3 Calculating likelihoods}

Likelihoods are calculated using light-based longitude estimates, SST and depth-temperature profiles (collected by the archival tag). For each time point, $t$ (here we use daily), we calculate 
a likelihood of the animal's position $\left(L\left(x_{(t)}\right)\right)$ on the grid:

$$
L\left(x_{(t)}\right)=L\left(x_{(t)}\right)_{1} \times L\left(x_{(t)}\right)_{2} \times \ldots L\left(x_{(t)}\right)_{n}
$$

where 1:n indicates individual likelihood components, which are formed for each type of input data at each time point (e.g. $\left.L_{S S T}\left(x_{(t)}\right)\right)$. Currently, HMMoce is built for $\mathrm{n} \leq 3$ because no combination of light, SST and depth-temperature profiles can sensibly be combined for $\mathrm{n}>3$ (i.e. cannot use both WOA and HYCOM likelihood at the same time).

\section{Light-based longitudinal likelihoods}

Tags record light levels from which sunrise and sunset times can be calculated (Hill and Braun, 2001). We calculate position estimates using Wildlife Computers GPE2 software, which allows vetting of spurious light measurements. The longitude and corresponding error estimate from GPE2 are used to evaluate a probability density function, resulting in a likelihood surface for this data. HMMoce also includes functionality for using latitude estimates (resulting in elliptical likelihood surfaces), but the default is longitude only.

\section{SST likelihoods}

For daily SST likelihoods, we construct a probability density function integrated between calculated limits (Le Bris et al., 2013) to incorporate observational errors (e.g. tag sensor error and/or near-surface SST measurements). We form a daily SST likelihood by integrating between limits for tag-measured SST $\left(S S T_{\min }, S S T_{\max }\right)$ that encompasses the range of SST values (including observation error) measured within that day:

$$
L_{S S T}\left(x_{(t)}\right)=\int_{S S T_{\min }}^{S S T_{\max }} N\left(t ; \mu_{z}, \sigma_{z}\right) d z
$$

where $N$ is a normal probability distribution function, $\mu_{z}$ the remotely-sensed SST grid cell value, and $\sigma_{z}$ the grid cell standard deviation. Standard deviation for remotely-sensed SST values are calculated using a "moving window" mean from raster: focal in $\mathrm{R}$ to incorporate approximately $0.25^{\circ}$ of SST data around each cell.

\section{Profile-based likelihoods}

Archival tags collect summaries of depth-temperature profiles at user-defined intervals throughout the deployment period. While these summaries vary in temporal and spatial resolution depending on study species, the profiles are a representation of a particular environment and time point during tag deployment that can be exploited in a spatial context. Tag profile data is recorded at various depth levels that often does not correspond to standard depth levels produced for the World Ocean Atlas or HYCOM. Thus, in all cases, we use linear 
regression in the $\mathrm{R}$ package locfit to predict profile temperatures from the tag at standard oceanographic depth levels in the corresponding dataset of interest. In addition, Wildlife Computers tags collected daily minimum and maximum temperatures at all depth levels which yield a thermal envelope of possible temperatures (rather than a single profile). In all cases, we construct a probability density function integrated between calculated limits (Le Bris et al., 2013) (e.g. minimum and maximum temperature), and standard deviation is calculated using a "moving window" as described previously for SST likelihood. Likelihoods based on tag profile data can be constructed using 3 different approaches.

Ocean Heat Content Ocean heat content $(\mathrm{OHC})$ is an integrated measure of temperature in the upper ocean that has been used by oceanographers and meteorologists since the mid20th century (Palmen, 1948) and may reveal complex structuring in the upper ocean at the mesoscale (Luo et al., 2015). OHC is calculated by integrating the thermal energy above an isotherm depth $(D)$ to the surface as:

$$
O H C=c_{p} \rho \int_{D}^{0}\left(T_{z}-T_{D}\right) d z
$$

where, $c_{p}$ is the specific heat constant of water $\left(3.993 \frac{\mathrm{kJ}}{\mathrm{kg}} \mathrm{C}\right), \rho$ is sea water density $(1025$ $\left.\frac{\mathrm{kg}}{\mathrm{m}^{3}}\right), T_{z}$ is water temperature $\left({ }^{\circ} \mathrm{C}\right)$ at depth $z$ and $T_{D}$ is water temperature at isotherm depth $D$. This results in a single minimum and maximum OHC metric for each day of tag profiles (see $\min / \max$ discussion above) between which a probability density function is integrated to incorporate the range of possible tag-based $\mathrm{OHC}$ values. $\mathrm{OHC}$ calculations are performed on daily $0.08^{\circ} \mathrm{HYCOM}$ grids which form the environmental grids used to compare tag observations to in the probability density framework:

$$
L_{O H C}\left(x_{(t)}\right)=\int_{O H C_{\min }}^{O H C_{\max }} N\left(t ; \mu_{z}, \sigma_{z}\right) d z
$$

where $N$ is a normal probability distribution function, $\mu_{z}$ the $\mathrm{OHC}$ grid cell value calculated from a daily HYCOM grid and $\sigma_{z}$ the grid cell standard deviation.

World Ocean Atlas and HYCOM The World Ocean Atlas 2013 contains a climatological monthly mean temperature for the global ocean from in situ profile data on a $1 / 4^{\circ}$ or $1^{\circ}$ grid (Locarnini et al., 2013). Tag-based minimum and maximum temperature profiles (after regression to predict values at standard WOA depth levels) are compared to WOA grids at standard depth levels to generate a likelihood surface at individual depth levels, $n$ :

$$
L_{W O A}\left(x_{(t)}\right)_{n}=\int_{T_{\min n}}^{T_{\max n}} N\left(t ; \mu_{z}, \sigma_{z}\right) d z
$$

where $N$ is a normal probability distribution function, $\mu_{z}$ the WOA grid cell temperature at depth $n$ and $\sigma_{z}$ the temperature standard deviation at depth $n$. Then the product 
of likelihood surfaces at each depth level $(1: n)$ is calculated to generate a single depthtemperature profile likelihood for time $t$ :

$$
L_{W O A}\left(x_{(t)}\right)=L_{W O A}\left(x_{(t)}\right)_{1} \times L_{W O A}\left(x_{(t)}\right)_{2} \times \ldots L_{W O A}\left(x_{(t)}\right)_{n}
$$

The same process is repeated to calculate depth-temperature profile likelihoods using HYCOM as the environmental grid to formulate $L_{H Y C O M}\left(x_{(t)}\right)$.

\section{A.1.4 Model formulation}

Details of model formulation, estimation and selection for HMMoce are derived from Pedersen et al. (2011a) and are discussed there in depth. Briefly, the movement of an animal is considered as a Brownian motion which, given the current behavior state $I_{t}$ of the animal, is decribed by a diffusivity matrix $D_{I_{t}}$ with unit $\mathrm{km}^{2}$ day ${ }^{-1}$. The application of diffusion processes in animal movement modeling is well established for analysis of tag data (e.g. Sibert et al., 2003; Pedersen et al., 2008) and more broadly (Okubo and Levin, 2013). We consider the joint process of movement and behavioral states as a probability density $\varphi_{i}(x, y, t)$ which describes the probability that the animal at time $t$ is located at $(x, y)$ and is in behavioral state $i$. The time evolution of the probability density of a particle performing brownian motion follows a diffusion-advection equation which can be augmented to include behavior switching dynamics (see Eqn. 1, Pedersen et al., 2011b). Our approach uses numerical approximation to solve an advection-diffusion equation by discretizing the continuous statespace into a grid (Thygesen et al., 2009). In discrete state-space, the probability density can be represented by a vector, $\varphi$, containing the state probabilities $(x, y, i)$ through time.

Model estimation is conducted in a HMM framework using a filtering approach (Zucchini and MacDonald, 2009) that yields the probability distribution of states moving forward in time conditional on observations, $\varphi\left(t_{k} \mid Z_{k}\right)$. The filter recursion alternates between time and data updates and returns a likelihood measure that can be used for parameter estimation and model selection. The final step includes a HMM smoothing step (Thygesen et al., 2009) that works backwards in time on filtered state estimates, thus leveraging the full dataset. This step provides the posterior probability distribution of each state (e.g. location and behavior) at specific times (typically daily). For more details on model formulation, see Pedersen et al. (2008) and Pedersen et al. (2011b) and references therein.

Most of the HMM filtering and smoothing mechanics remain unchanged from that developed by Pedersen et al. (2008); however, two main improvements are worth noting. First, image processing techniques are used in the convolution steps at each time point in the filter (see ?hmm.filter) to make the convolution more intuitive and improve computation speed. For full details of the convolution, see ?imager : convolve (Barthelme, 2016). Second, we add a masking technique within the filtering step that allows the user to set boundary thresh- 
olds on how much of the observation likelihood grid is used in the filter step. This masking approach can be used to set a constant distance, or a percent threshold of the bounding box, from the previous day's output to help constrain the following day's results. This approach

is still experimental but may help constrain position estimates by weighting regions of the observation likelihood more highly if they are closer to the previous days filter probability.

\section{Parameter Estimation}

The filtering process above returns a likelihood measure which indicates how well the model fits the data. Thus, this likelihood function of the unknown parameters $\theta$ (e.g. diffusion, switching rates) can be evaluated at, say, $\theta_{0}$ by running the filter using the parameter values in $\theta_{0}$. Maximum likelihood (ML) estimation of model parameters is then straight forward and can be performed by most standard numerical optimizers. However, recent work by Woillez et al. (2016) further exploited the properties of the discretization of space in this model and addressed a joint ML estimation of all model parameters using an iterative Expectation-Maximization (EM) framework.

Here the EM algorithm is a two-step iterative procedure: the expectation (E) step computes the posterior distribution $P\left(\left(X_{t}\right)_{t=0: N-1} \mid\left(Y_{t}\right)_{t=0: N-1}\right)$ given current model parameter estimates, $\theta_{v}$; and the maximization (M) step updates model parameters $\left(\theta_{v+1}\right)$ according to a ML criterion reweighted by the posterior distribution. Currently in HMMoce, $\theta_{v}$ represents transition probability between states, while movement parameters are fixed to realistic estimates of diffusion as determined by estimated tag speeds calculated from Argos-based positions or based on expert knowledge. The theoretical movement process is considered purely Brownian, and advection is currently not considered. The two-step EM procedure for state-switching probabilities is iterated through $\theta_{N}$ until a convergence threshold is met. Switching rates are calculated in an EM framework following Woillez et al. (2016) (see expmax function in HMMoce) with a change threshold that defaults to $1 \%$.

\section{Model results}

There are a number of ways the posterior distribution of the state can be used to represent animal movements. Approaches in the literature have been to calculate the mean or mode across the distributions through time (Pedersen et al., 2008, 2011b) to represent a most probable track. A third approach to estimate most probable tracks from the posteriors is using the Viterbi algorithm (Viterbi, 2006) which has been used in movement ecology (Pedersen et al., 2008; Thygesen et al., 2009; Winship et al., 2012; Jonsen et al., 2013) but is extremely computationally intensive. HMMoce currently has functionality for both mean and mode.

Summing the posterior distribution over time across all behaviors yields a distributional estimate of residence time (Pedersen et al., 2011b) as well as yields insight on the cumulative 
distribution of the animal's residency (utilization distribution). Similarly, behavior state estimates can be summed over space to get the probability of the animal being in each behavioral state through time and across the recorded movements and can be used to detect differential space use (e.g. Figure 2-4 in the main text).

\section{A.1.5 Case study methods}

\section{Tagging methodology}

Three blue sharks and one shortfin mako were tagged near Cape Cod, MA during Fall 2015 with both pop-up satellite archival transmitting (PSAT) tags and Argos-based SPOT tags. Sharks were caught on rod and reel and then brought onboard the fishing vessel. We attached satellite-linked radio telemetry tags (Wildlife Computers finmount SPOT5 tags) to the dorsal fin with nylon surgical hardware. PSAT tags were attached to a short $(<20$ $\mathrm{cm}$ ) stainless steel tether that terminated in a titanium anchor. The anchor was inserted into the dorsal musculature near the base of the dorsal fin. PSATs were programmed to report summarized daily depth-temperature profiles, sunrise and sunset event timing and sea surface temperature and to pop-up 180 days after tagging. The SPOT tag collected Doppler-based geopositions from Argos satellites when a wet/dry switch on the tag was activated at the surface-air interface. Resulting locations were processed with a Kalman filtering algorithm by Collecte Localisation Satellites (Lopez et al., 2014) and subsequently assigned error flags called location classes (LC): LC 3, <250 m; LC 2, 250-500 m; LC 1, 500-1500 m; LC 0, >1500 m for classes 3, 2, 1, 0. Additional classes A, B represented positions derived from less than 4 satellite messages and were not assigned an estimated spatial accuracy. Location class $\mathrm{Z}$ positions were considered invalid and removed from further analysis (CLS, 2016). After Z class positions were removed, the remaining positions were filtered using a speed filter $\left(2 \mathrm{~ms}^{-1}\right)$ from the trip package (Sumner and Luque, 2015), based on characteristic speeds (Figure A-1), and regularized to daily positions using crawl (Johnson et al., 2008) in R (R Development Core Team, 2015). Results metrics from various HMMoce model runs (referred to in the main text) are shown in Table A-1. One complete blue shark dataset is included as example data in the package, and its analysis is described in full detail in the package vignette.

\section{Grid resolution and time trials}

Our method uses grid-based computations as they afford both flexibility and computational improvements, but changes in grid size can alter likelihood surfaces, model results and computation time. Several trials of HMMoce were conducted on an Amazon EC2 instance (2.3 GHz Intel Xeon processors, 64 CPUs, $256 \mathrm{GiB}$ memory) and a consumer grade desktop computer (2.53 GHz processors, 4 CPUs, $12 \mathrm{GiB}$ memory) encompassing various grid sizes. 
HMMoce modeling was initially performed on $0.25^{\circ}$ grids, including likelihoods and model selection, to match the grid-based calculations conducted in GPE3 (WC, 2015), but the resolution was increased to a $0.08^{\circ}$ grid for the final model runs for each tag dataset. Results from time trial and grid resolution model runs indicated that higher resolution grids (as fine as $\left.0.08^{\circ}\right)$ yielded roughly equivalent results to slightly more coarse $\left(0.25^{\circ}\right)$ grids while significantly increasing computation time (approx. 3-fold increase) (Table A-2). However, grids as coarse as $1^{\circ}$ often yielded poor fit metrics (10-fold increase in error), likely as a result of the loss of mesoscale variability in oceanographic data at this resolution.

\section{Data removal experiment}

While HMMoce was designed to improve geolocation estimates for all tagged marine organisms, the main impetus for the work was to fulfill a need for improving track estimates in cases where light and SST data (from minimal surface occupation) were lacking. In order to use the double-tagged shark dataset (with high quality light and SST data) to test this, we randomly removed (using sample in base R, without replacement) $75 \%$ and $50 \%$ of deployment days with adequate light and SST data, respectively, for geolocation from the shark PSAT data (while keeping the depth-temperature profile data for these days). The removals approximated PSAT data quality typical of swordfish tag deployments in the Atlantic Ocean due to crepuscular diving behavior and light avoidance (Braun et al., 2015a; Neilson et al., 2009, Chapter 2). The resulting tracks were treated as independent from track model runs performed on the complete datasets, and HMMoce likelihood routines and GPE3 track construction methods were unchanged.

\section{A.1.6 Other geolocation models}

Almost all of the available geolocation approaches for archival tag data rely on light or a combination of light, SST and a bathymetric mask that is often applied ad hoc. Light-based positioning is predicated on an accurate measure of day length. Yet, many species rarely, if ever, visit the surface layer during the appropriate periods required for calculation of sunrise and sunset times (e.g. swordfish, Neilson et al., 2009; basking sharks, Skomal et al., 2009). Thus, we sought to develop a modeling framework that would also be applicable to species with limited light data.

To facilitate comparison of HMMoce with existing methods, we used two existing statespace models that have been widely used in marine biotelemetry (e.g. Braun et al., 2015b; Galuardi and Lutcavage, 2012; Lam et al., 2014, 2016; Thorrold et al., 2014; Winship et al., 2012): a sea surface temperature inclusive Unscented Kalman filter, Ukfsst, (Lam et al., 2008; Nielsen and Sibert, 2007) and a coherent state-space model using raw light levels recorded on the tag, Trackit, (Nielsen and Sibert, 2007). Sea surface temperature fields used in Ukfsst were weekly, $1^{\circ}$ optimally interpolated values (Reynolds et al., 2002). Initial values 
for parameters estimated in both models were adjusted and/or flagged as inactive in the estimation until a convergence minimum was reached. A second stage bathymetric correction was applied to the estimated Ukfsst tracks to improve quality, particularly near landmasses (Galuardi et al., 2010). A global, $1^{\circ}$ product, E-Topo1 (Amante and Eakins, 2009), was used for this step. For each model estimate, the most probable track was used for comparison. While Trackit and Ukfsst are built on a fundamentally different statistical framework than the HMM models, they have been widely used in the same way to estimate movements of individuals using data from archival tag deployments. While neither Ukfsst or Trackit may have been fully optimized here, their inclusion allowed a test of the potential benefits of using 3D tag data for marine biotelemetry, particularly when quality light and SST data is lacking. We also compared our results to a proprietary, manufacturer-specific (Wildlife Computers) HMM called GPE3. The GPE3 algorithm does not permit customization of model details except "typical animal speed." Speed estimates used to generate GPE3 tracks were 2 or $4 \mathrm{~m} / \mathrm{s}$ to match speeds used in HMMoce based on speeds of Argos-tracked sharks (Figure A-1). This comparison is discussed in detail in the main text. Resulting tracks from the model comparison and the HMMoce most probable track with behavior switching results are shown for the mako (Figure 2-2 Figure 2-3, respectively) and the blue sharks (Figure A-2, Figure A-3, Figure A-4) for blue shark model comparison and Figure A-6, Figure A-7, Figure A-8 for HMMoce track and behavior switching results). Discussion of model limitations and an example comparison to HYCOM, WOA and OHC likelihoods is discussed in the main text and shown in Figure A-5.

Reported error metrics throughout this study include great-circle distance between true (Argos) and estimated locations on the Earth's surface using the rdist.earth.vec function in the fields (Nychka et al., 2015) package for $\mathrm{R}$ and root-mean-square error (RMSE) between daily model output and Argos locations (Galuardi et al., 2008; Wilson et al., 2007; Braun et al., 2015b). 


\section{A.2 Supplemental Figures}

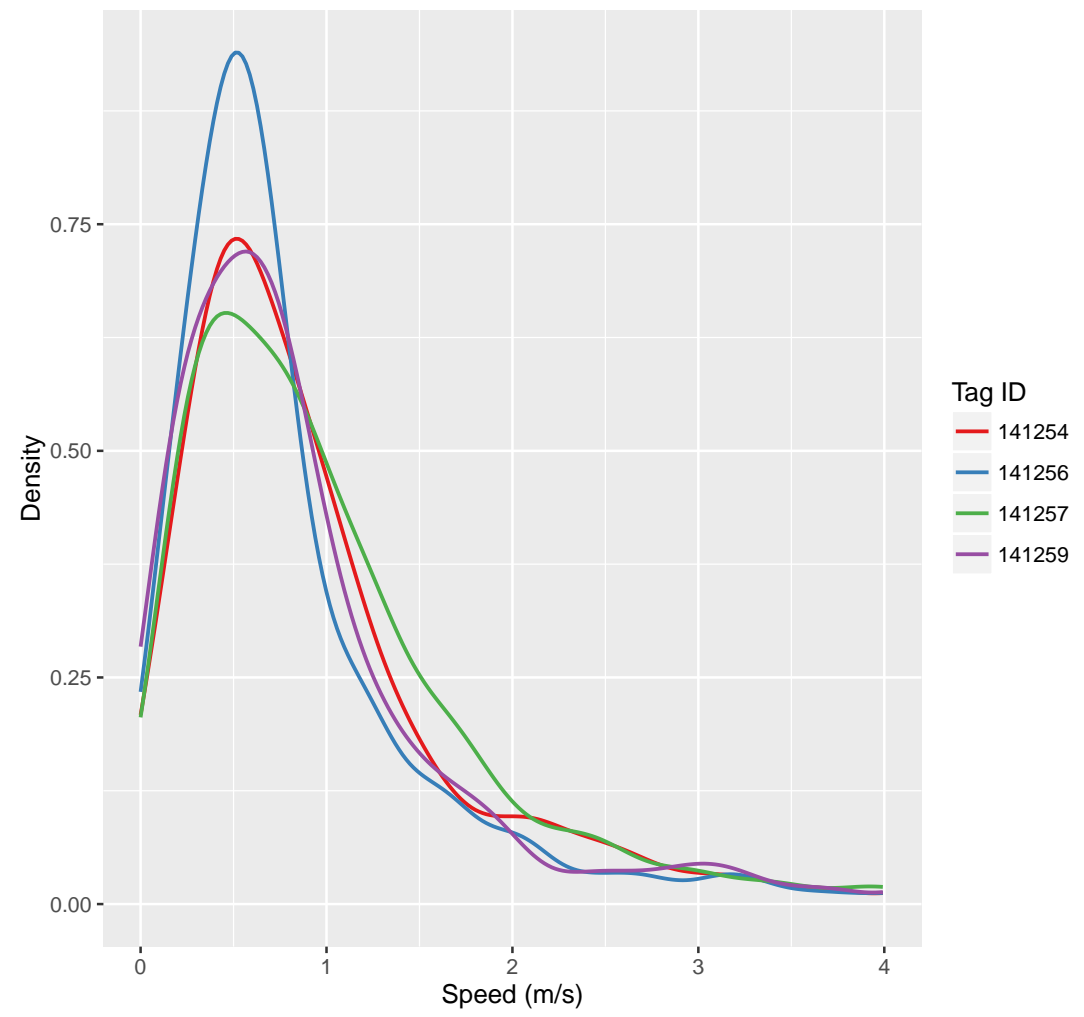

Figure A-1: Density of speeds among Argos-based positions for tagged individuals. 

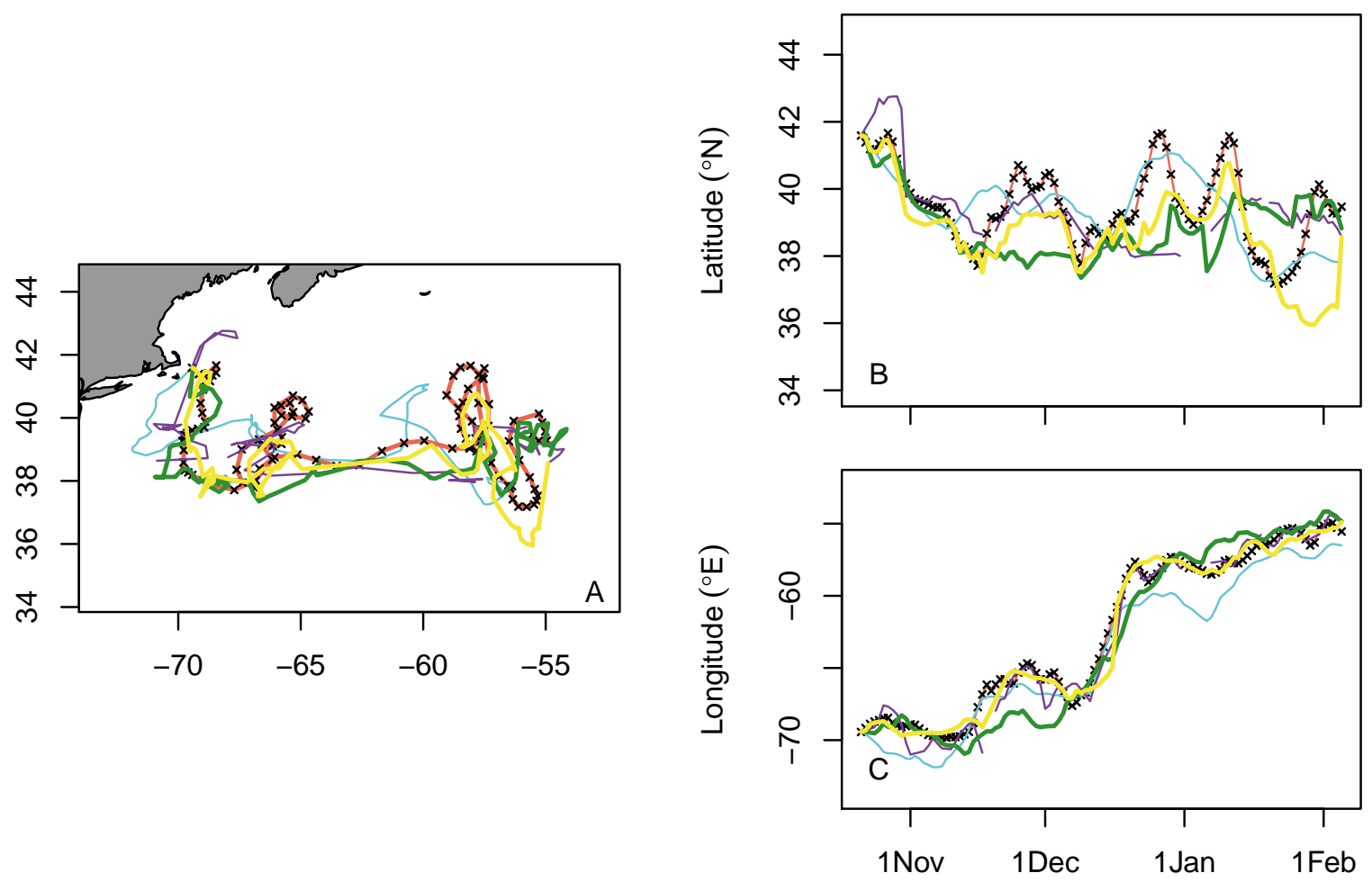

Figure A-2: Calculated tracks for blue shark 141254 using the 4 different geolocation approaches (Ukfsst, purple; Trackit, blue; GPE3, green; HMMoce, yellow) compared to the "known" Argos-based track (red, black crosses). Latitudinal and longitudinal estimates through time are shown in panels $\mathrm{B}$ and $\mathrm{C}$, respectively. Lines appear broken when a resulting track is missing daily data. 

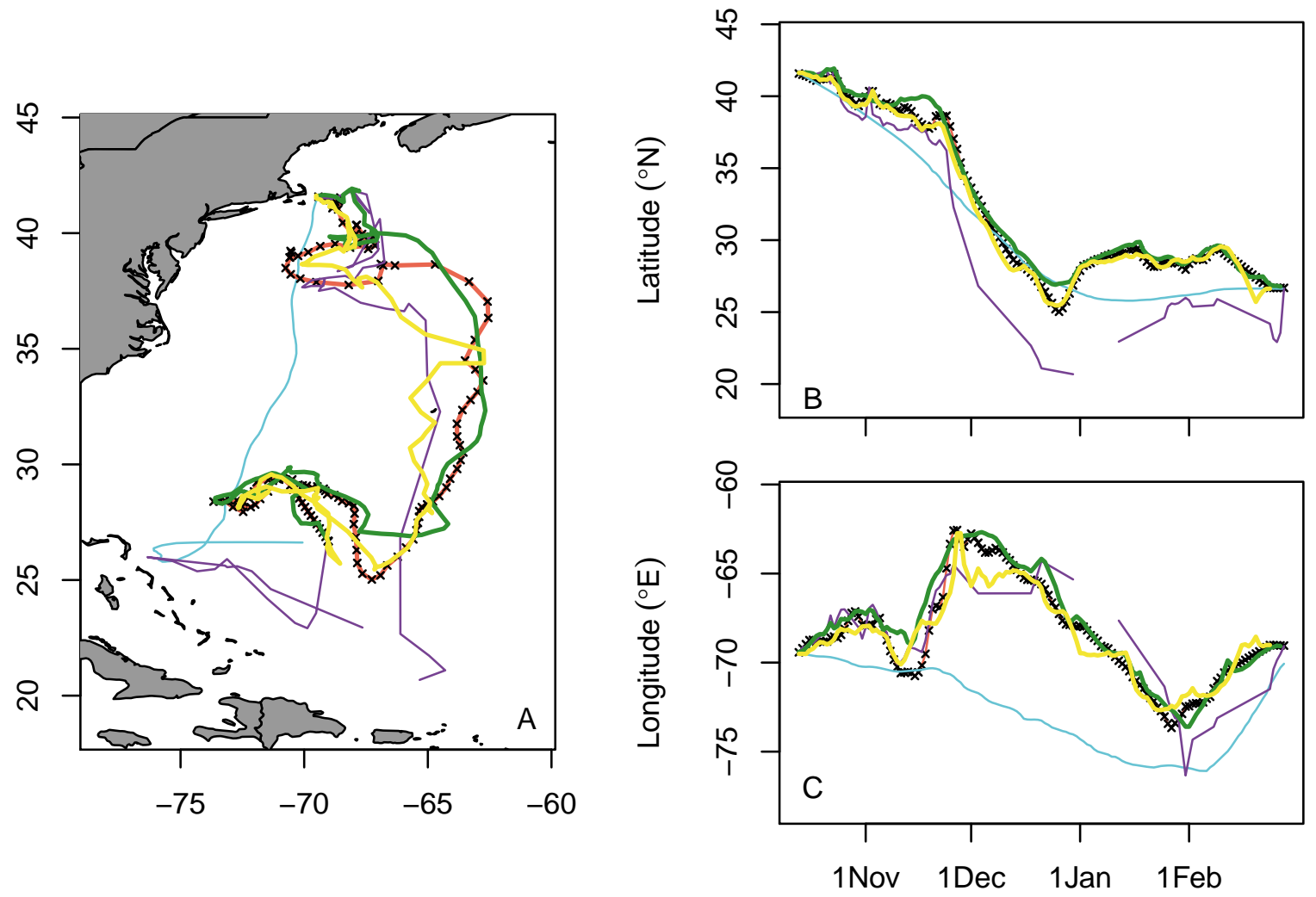

Figure A-3: Calculated tracks for blue shark 141256 using the 4 different geolocation approaches (Ukfsst, purple; Trackit, blue; GPE3, green; HMMoce, yellow) compared to the "known" Argos-based track (red, black crosses). Latitudinal and longitudinal position estimates through time are shown in panels B and C, respectively. Lines appear broken when a resulting track is missing daily data. 

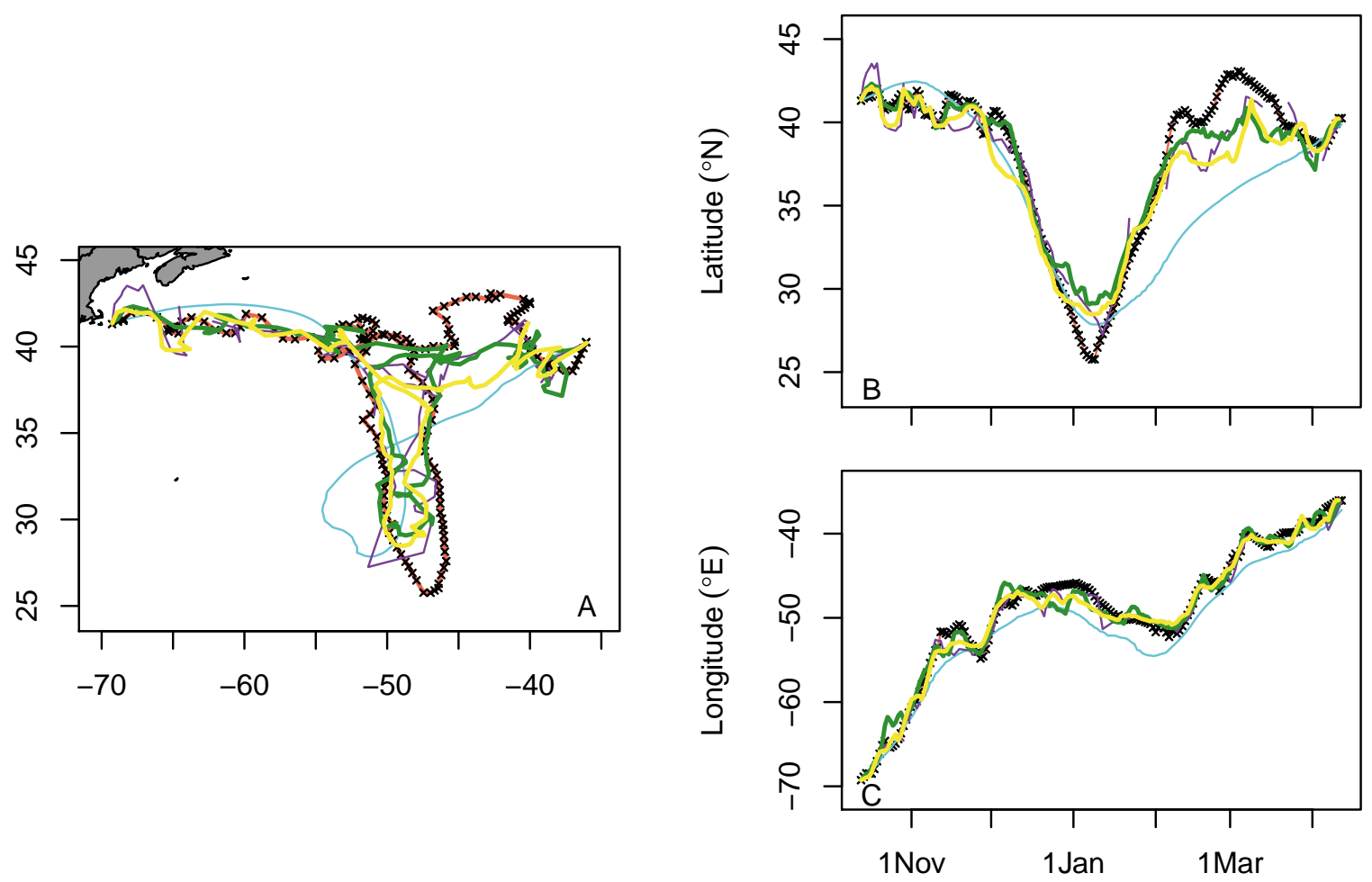

Figure A-4: Calculated tracks for blue shark 141259 using the 4 different geolocation approaches (Ukfsst, purple; Trackit, blue; GPE3, green; HMMoce, yellow) compared to the "known" Argos-based track (red, black crosses). Latitudinal and longitudinal position estimates through time are shown in panels $\mathrm{B}$ and $\mathrm{C}$, respectively. Lines appear broken when a resulting track is missing daily data. 

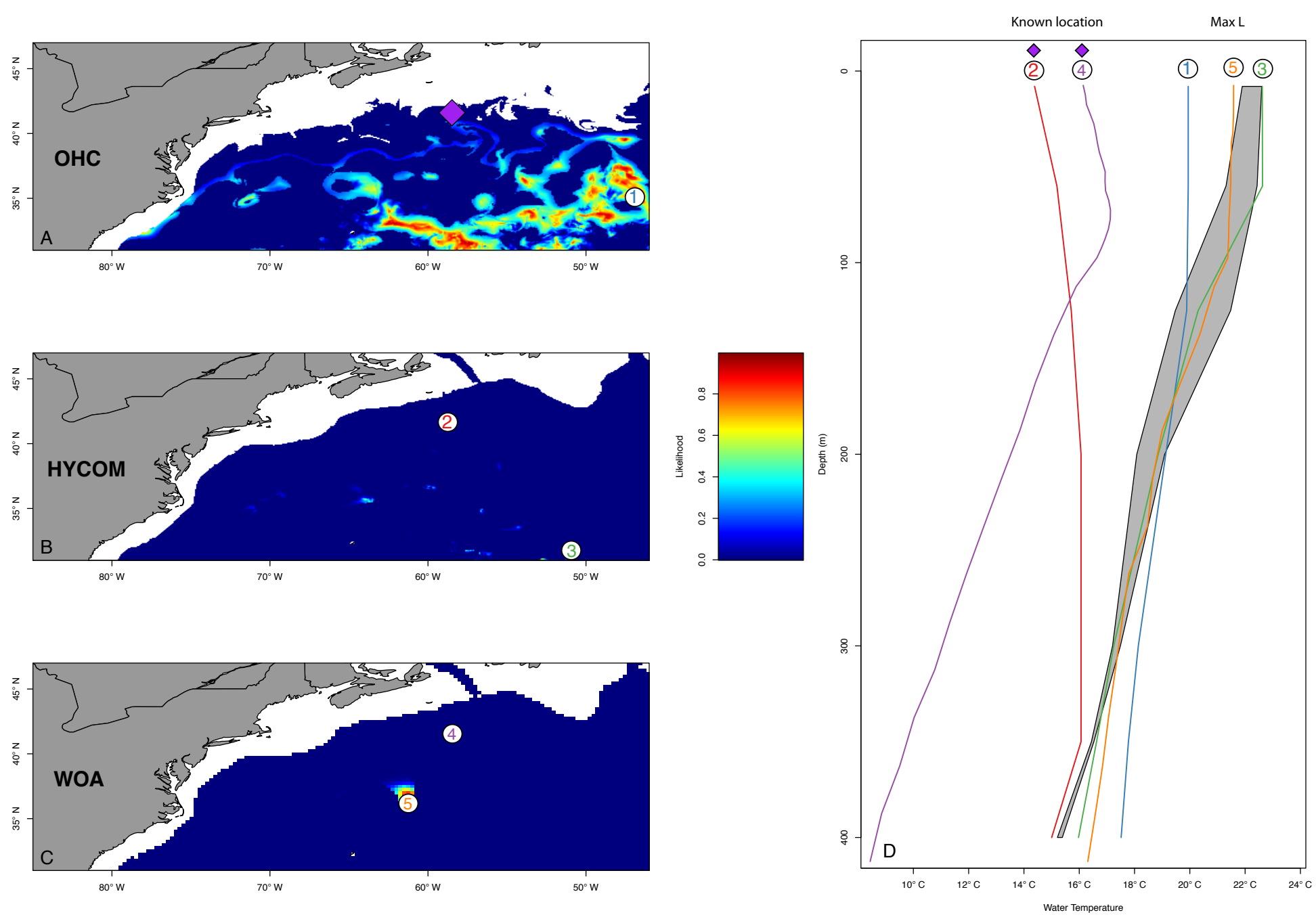

Figure A-5: Example depth-temperature profile-based likelihoods for ocean heat content (OHC), HYCOM and World Ocean Atlas (WOA)(panels A-C) for a given day of observation. Numbers correspond to the location where each depth-temperature profile (panel D) was taken. Purple diamond indicates known position for this example day. Profiles 2 and 4 correspond to profiles extracted from HYCOM and WOA, respectively, at the known location of the tag. In comparison, profiles 1, 3 and 5 indicate profiles taken from the grid cell containing the maximum likelihood, as calculated by HMMoce, for OHC, HYCOM and WOA, respectively. Grey shaded region in right panel indicates depth-temperature envelope measured by PSAT tag. This figure demonstrates an example day in which the measured PSAT tag data was compared to environmental grids to generate maximum likelihoods, but the resulting likelihoods exhibited significant error when compared to independent, known positions and profiles extracted at those locations. 

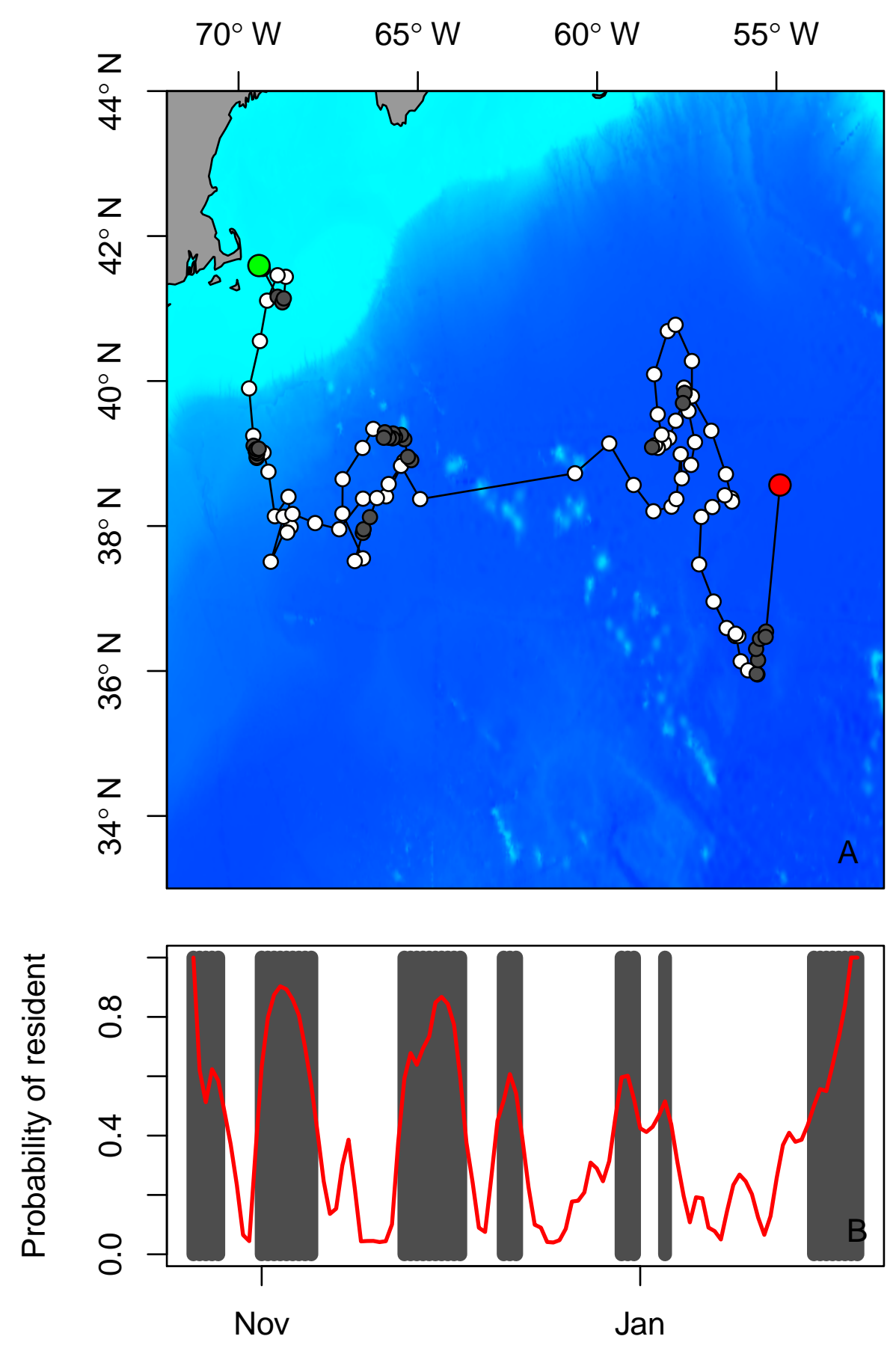

Figure A-6: Movements (A) and behavior (B) calculated using HMMoce for blue shark 141254. The tagged individual is considered resident where probability of residency is greather than 0.5 (grey points and bars in panels A and B, respectively). Green and red points indicate tag and pop-up location respectively. Black line follows predicted daily locations of tagged shark. 

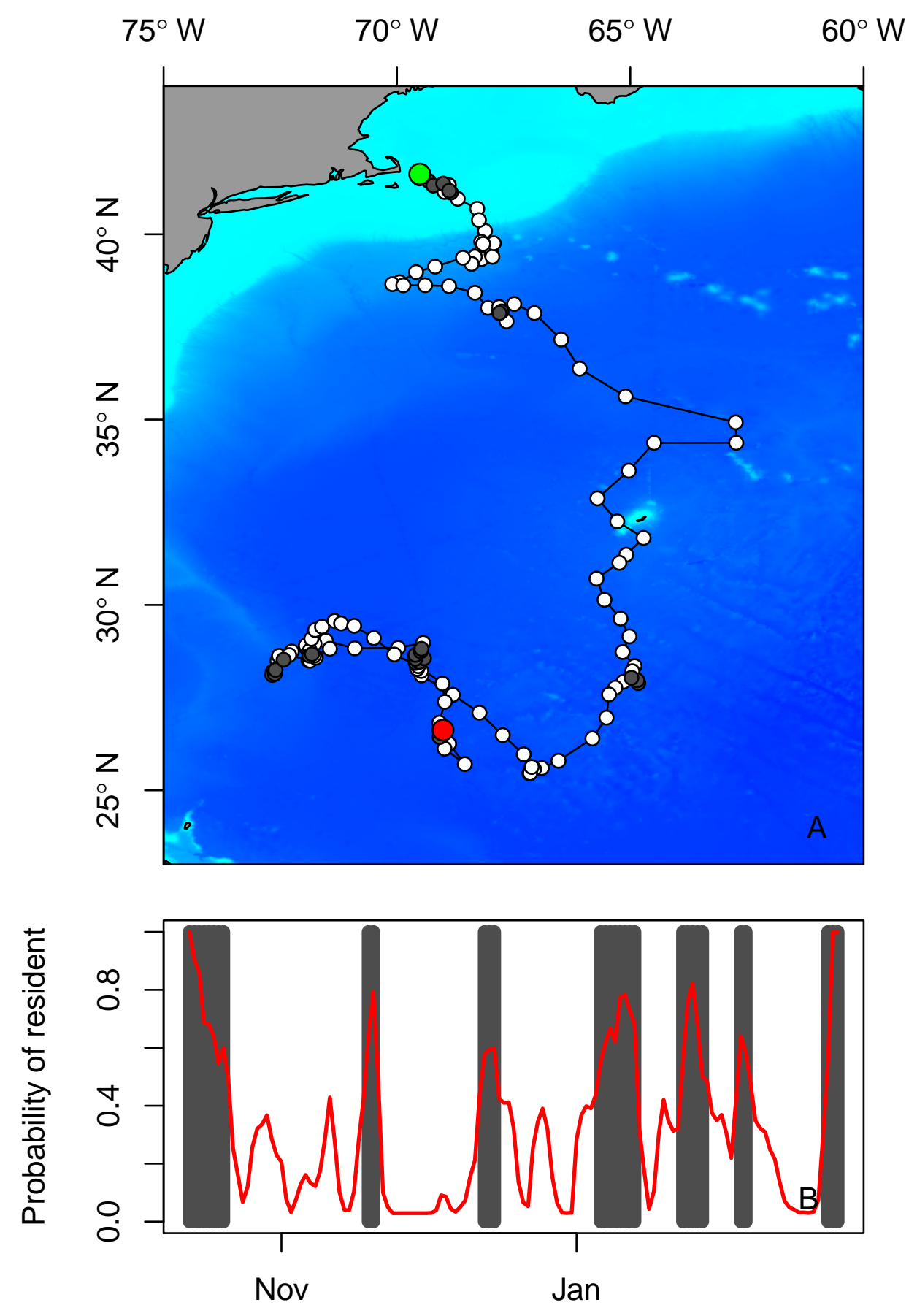

Figure A-7: Movements (A) and behavior (B) calculated using HMMoce for blue shark 141256. The tagged individual is considered resident where probability of residency is greather than 0.5 (grey points and bars in panels A and B, respectively). Green and red points indicate tag and pop-up location respectively. Black line follows predicted daily locations of tagged shark. 

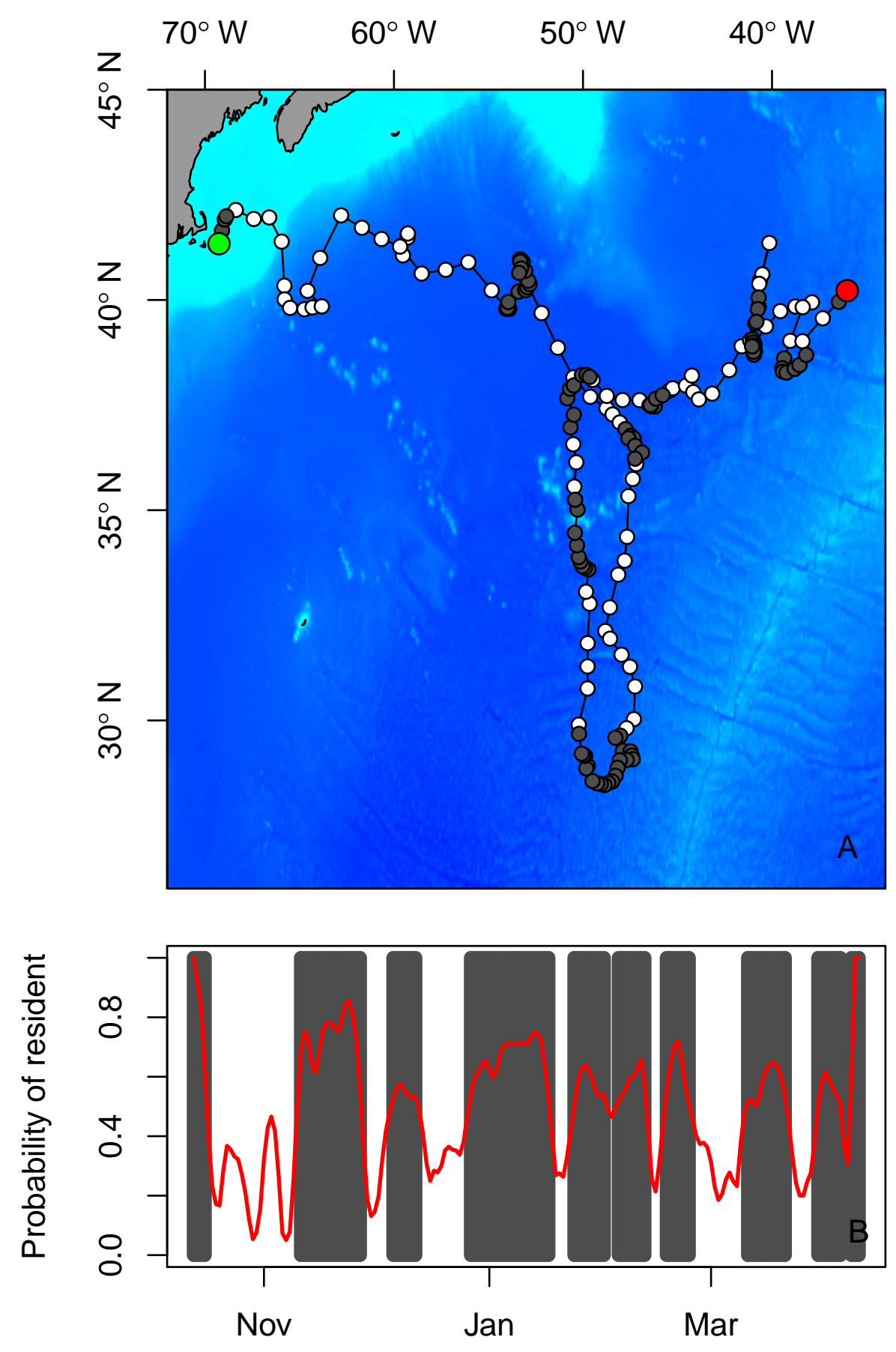

Figure A-8: Movements (A) and behavior (B) calculated using HMMoce for blue shark 141259. The tagged individual is considered resident where probability of residency is greather than 0.5 (grey points and bars in panels A and B, respectively). Green and red points indicate tag and pop-up location respectively. Black line follows predicted daily locations of tagged shark. 


\section{A.3 Supplemental Tables}

Table A-1: Example error metrics for a HMMoce model run of each observation likelihood input: light (L), SST (S), ocean heat content (O), World Ocean Atlas profiles (W) and HYCOM profiles (H). Root-mean-square error (RMSE) is shown for Latitude, Longitude in degrees. Reported mean and standard deviation values are based on pointwise distance calculations from known positions $(\mathrm{km})$. P1 and P2 indicate the calculated probability of state-switching from the Expectation-Maximization algorithm where, in this case, $\mathrm{P} 1$ is the probability of remaining in a migratory state (implemented in a migratory movement kernel for the convolution). P2 represents a resident behavior state. Migratory speeds were fixed at $2 \mathrm{~m} / \mathrm{s}$ on a $0.08^{\circ}$ grid for the example run.

\begin{tabular}{|c|c|c|c|c|c|c|c|}
\hline Species & PTT & Input & RMSE & Mean & SD & $\mathrm{P} 1$ & P2 \\
\hline \multirow{7}{*}{$\mathrm{BSH}$} & \multirow{7}{*}{141254} & $\mathrm{LS}$ & $1.52,0.96$ & 143.5 & 123.3 & 0.97 & 0.86 \\
\hline & & $\mathrm{LO}$ & $3.09,1.03$ & 265.0 & 238.1 & 0.94 & 0.78 \\
\hline & & LW & $2.59,2.52$ & 303.9 & 199.6 & 0.98 & 0.82 \\
\hline & & $\mathrm{LH}$ & $3.09,1.43$ & 285.7 & 229.5 & 0.97 & 0.84 \\
\hline & & LSO & $1.21,0.81$ & 117.8 & 96.8 & 0.94 & 0.83 \\
\hline & & LSW & $2.37,2.73$ & 288.4 & 209.1 & 0.96 & 0.69 \\
\hline & & LSH & $2.05,1.2$ & 186.0 & 168.7 & 0.98 & 0.86 \\
\hline \multirow[t]{7}{*}{ BSH } & \multirow[t]{7}{*}{141256} & LS & $0.42,1.08$ & 88.7 & 66.5 & 0.98 & 0.90 \\
\hline & & $\mathrm{LO}$ & $1.7,1.03$ & 169.1 & 132.9 & 0.97 & 0.83 \\
\hline & & LW & $1.23,5.38$ & 444.7 & 320.4 & 0.98 & 0.73 \\
\hline & & $\mathrm{LH}$ & $0.59,1.15$ & 109.4 & 64.9 & 0.96 & 0.83 \\
\hline & & LSO & $1.14,2.61$ & 190.0 & 216.8 & 0.96 & 0.76 \\
\hline & & LSW & $0.93,4.67$ & 353.6 & 302.8 & 0.99 & 0.86 \\
\hline & & LSH & $0.55,1.03$ & 91.4 & 68.2 & 0.95 & 0.81 \\
\hline \multirow[t]{7}{*}{ BSH } & \multirow[t]{7}{*}{141259} & LS & $1.87,1.31$ & 196.7 & 140.0 & 0.99 & 0.82 \\
\hline & & $\mathrm{LO}$ & $2.77,1.17$ & 277.4 & 173.9 & 0.99 & 0.82 \\
\hline & & LW & $5.71,1.67$ & 525.4 & 391.5 & 0.96 & 0.71 \\
\hline & & $\mathrm{LH}$ & $2.37,1.55$ & 252.9 & 160.9 & 0.97 & 0.69 \\
\hline & & LSO & $2.01,1.31$ & 206.8 & 149.1 & 0.98 & 0.73 \\
\hline & & LSW & $2.52,1.87$ & 284.2 & 170.9 & 0.99 & 0.80 \\
\hline & & LSH & $2,1.36$ & 206.8 & 154.0 & 0.98 & 0.67 \\
\hline \multirow[t]{7}{*}{ MKO } & \multirow[t]{7}{*}{141257} & LS & $0.79,1.77$ & 132.4 & 119.5 & 0.93 & 0.94 \\
\hline & & $\mathrm{LO}$ & $1.81,2.03$ & 225.0 & 148.6 & 0.94 & 0.92 \\
\hline & & LW & $2.29,2.33$ & 284.9 & 164.4 & 0.95 & 0.78 \\
\hline & & $\mathrm{LH}$ & $1.26,2.22$ & 171.3 & 170.7 & 0.91 & 0.88 \\
\hline & & LSO & $0.82,1.58$ & 123.8 & 109.9 & 0.94 & 0.95 \\
\hline & & LSW & $1.72,2.29$ & 231.5 & 158.6 & 0.95 & 0.85 \\
\hline & & LSH & $1.08,2.03$ & 147.8 & 158.8 & 0.93 & 0.93 \\
\hline
\end{tabular}


Table A-2: Fit metrics and computation time (in mins) using an Amazon EC2 instance with 2.3 GHz processors and $64 \mathrm{CPUs}$ (High-performance computing; HPC) and a consumer-grade desktop computer with $2.53 \mathrm{GHz}$ processors and 4 CPUs (personal computer; PC) for blue shark 141256 . The $\mathrm{L}$ row indicates total run time for calculating individual likelihoods from light $\left(0.25^{\circ}\right.$ resolution $)$, sea surface temperature $\left(0.25^{\circ}\right)$, ocean heat content $\left(0.08^{\circ}\right)$ and HYCOM 3D profiles $\left(0.08^{\circ}\right)$ in the native resolution for each input environmental dataset. Environmental grids spanned $43^{\circ}$ of longitude and $45^{\circ}$ of latitude for this individual, resulting in a 539x564 matrix for daily OHC grids and daily HYCOM grids at each depth level (up to 35 depth levels to $1500 \mathrm{~m}$ depth) and $173 \times 181$ matrix for daily SST and light grids. The following rows indicate computation time for one complete iteration of the HMM (including filtering, smoothing and parameter estimation) computed at each resolution listed. The HMM computations are performed after the observation likelihoods have been calculated. Resolution is the grid resolution in degrees. Reported metrics are based on pointwise distance calculations from known positions. Mean and standard deviation (SD) are in kilometers and root-mean-square error is for Latitude, Longitude in degrees.

\begin{tabular}{lrrrrrr}
\hline Resolution & HPC Time & PC Time & Mean & SD & RMSE $_{\text {lat }}$ & RMSE $_{\text {lon }}$ \\
\hline L & 34.80 & 158.7 & & & & \\
0.08 & 23.90 & 1082.2 & 89.09 & 69.54 & 0.53 & 1.03 \\
0.25 & 8.08 & 148.2 & 117.81 & 118.78 & 0.48 & 1.61 \\
0.5 & 6.07 & 74.6 & 401.36 & 425.06 & 4.80 & 2.26 \\
0.75 & 5.52 & 20.3 & 867.37 & 453.36 & 5.16 & 6.95 \\
1 & 5.19 & 18.2 & 991.88 & 538.98 & 7.52 & 7.66 \\
\hline
\end{tabular}


THIS PAGE INTENTIONALLY LEFT BLANK 
Appendix B

ChAPTER 4 SuPPLEMENTAL INFORMATION 


\section{B.1 Supplemental Figures}

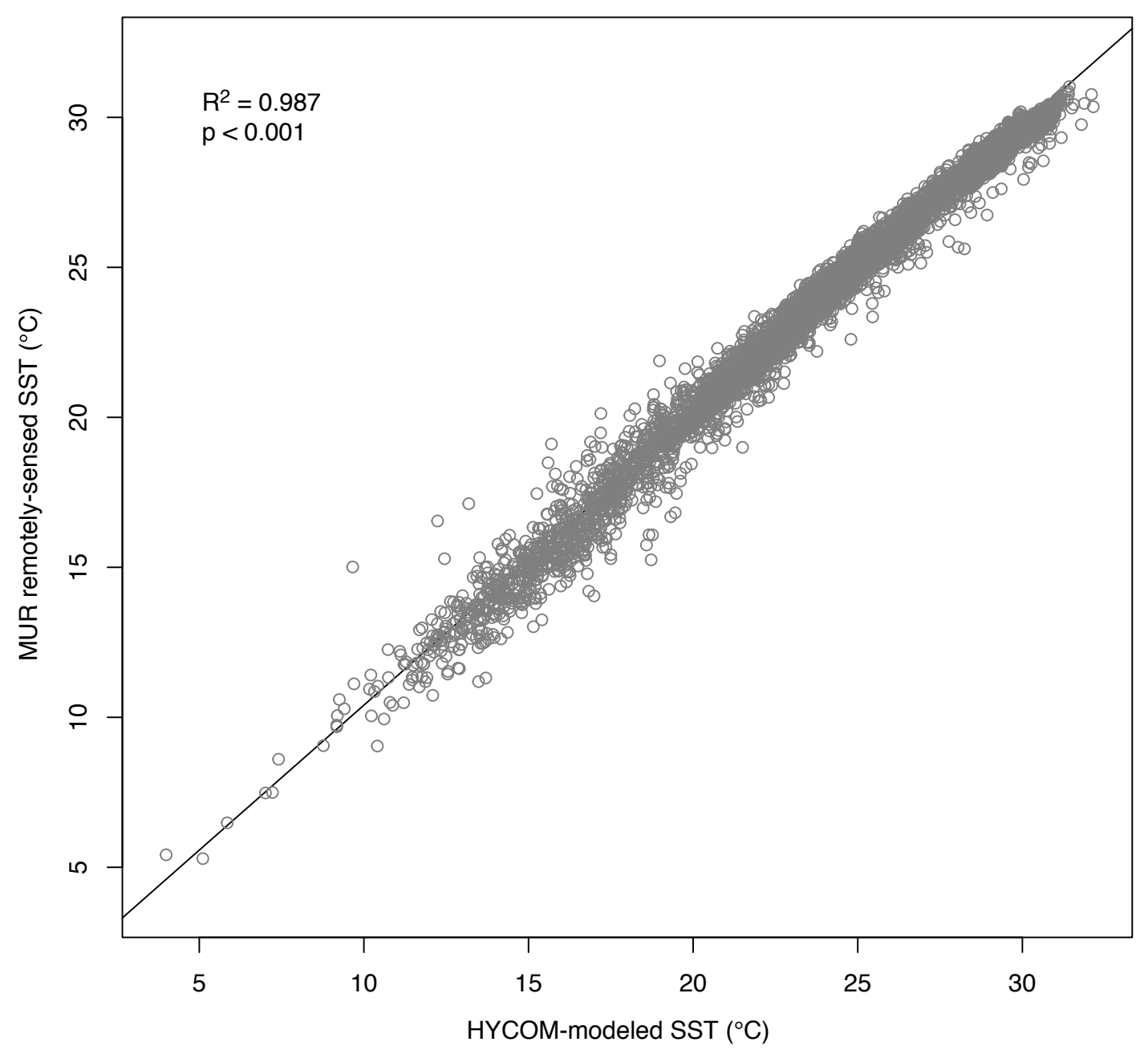

Figure B-1: Regression of MUR remotely-sensed SST and HYCOM (model-based) SST at a subset of swordfish conventional tag locations. 


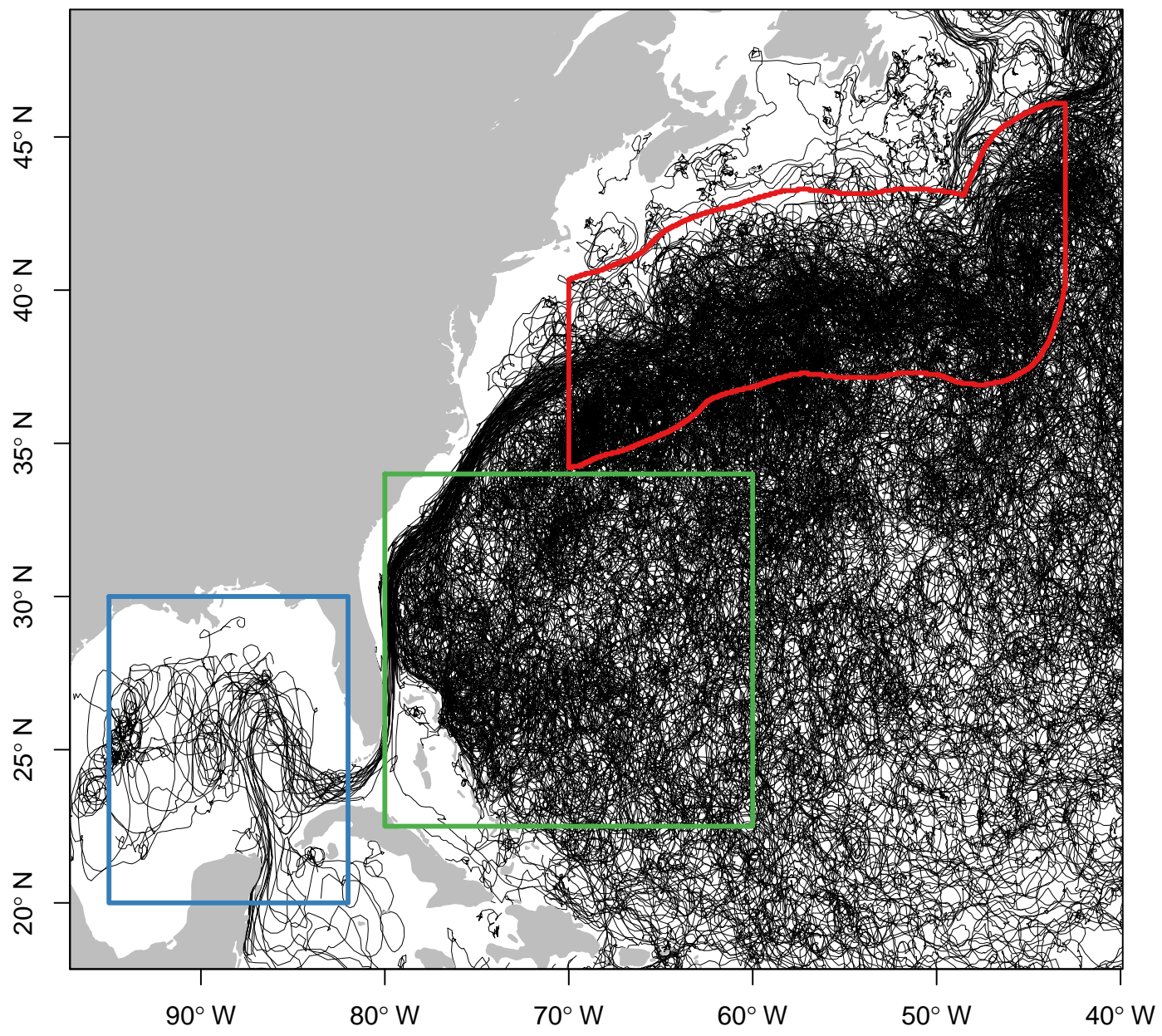

Figure B-2: Drifter trajectories used as null movements (black lines) and bounding boxes (colored lines) for the focal regions in the eddy collocation analysis. The data presented here has been trimmed to one location per day to aid visualization. Regions designate the Gulf Stream (red), Sargasso Sea (green) and Gulf of Mexico (blue). 

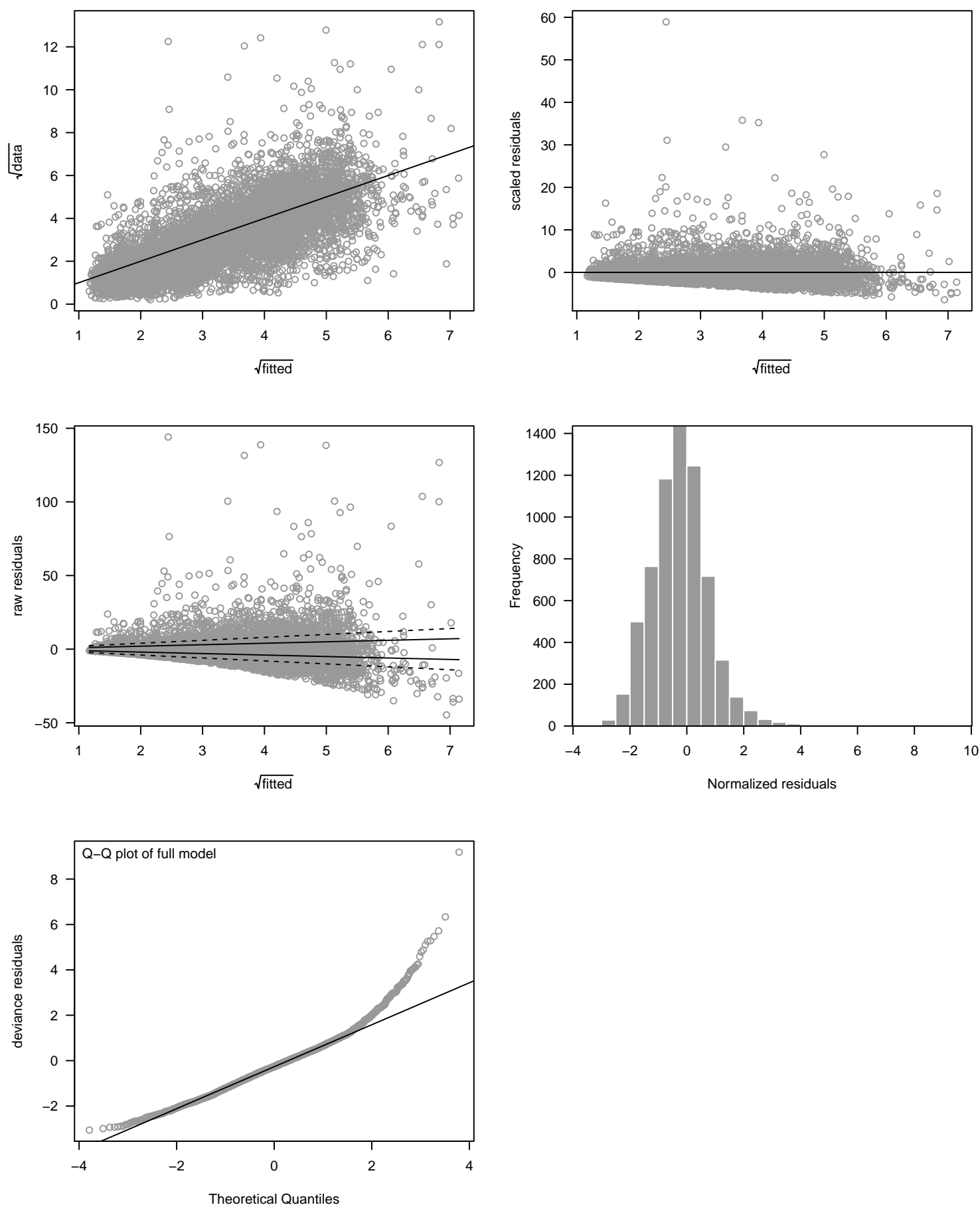

Figure B-3: Diagnostic plots for the final model from the generalized additive model analysis of catch-per-unit effort data. Code for generating these plots in R is from Lam et al. (2014). 


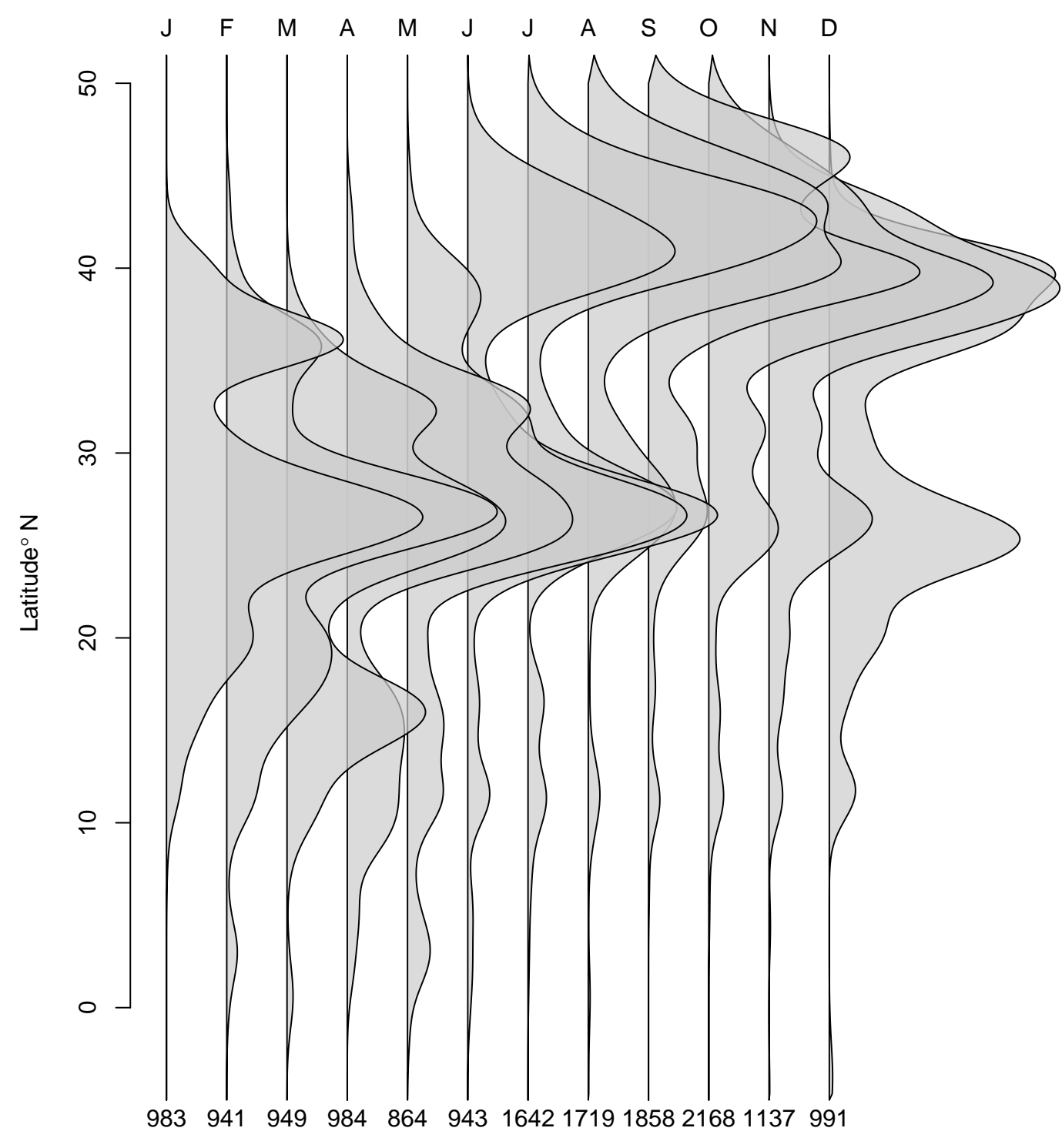

Figure B-4: Monthly density distribution of swordfish tagged with conventional tags by latitude. Upper letters indicate month and lower number labels indicate sample size of tagged individuals. 

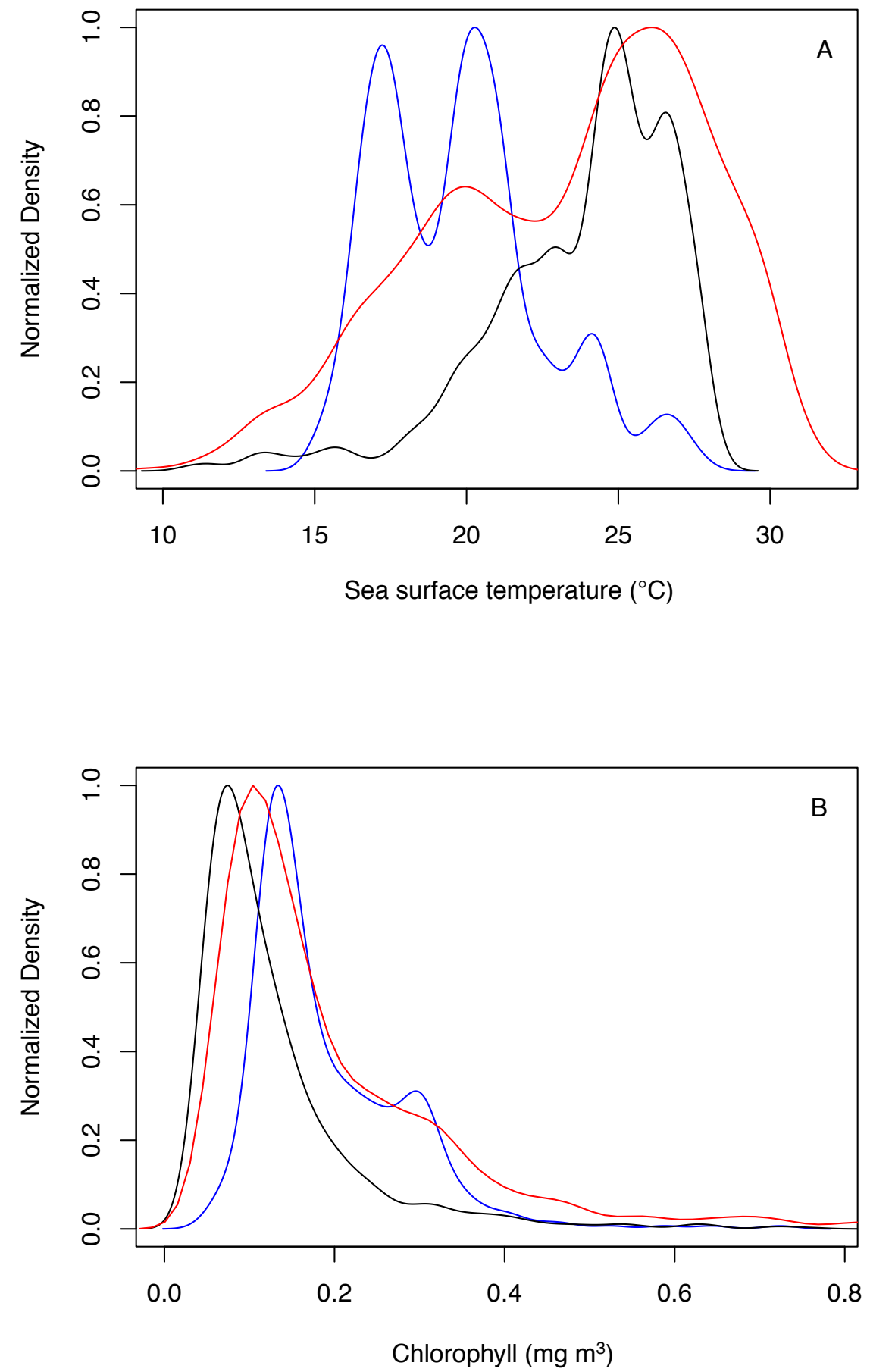

Figure B-5: Normalized density distributions of SST (A) and chlorophyll concentrations (B) experienced by satellite-tagged swordfish in the Azores (blue) and northwest Atlantic (red) and a subset of conventional-tagged swordfish in the North Atlantic (black) for which remote-sensing data was available. 
Appendix C

Chapter 5 Supplemental Information 


\section{C.1 Supplemental Methods}

\section{C.1.1 Satellite tagging and data}

Adult male blue sharks (mean $264 \mathrm{~cm}$ fork length, range 220-313 cm) were tagged near Montauk, New York during Summer 2013 and $2014(\mathrm{n}=2)$ and Cape Cod, MA during Fall 2015 and 2016 ( $\mathrm{n}=17$; Table C-1). All were tagged with fin-mounted Smart Position or Temperature Transmitting (SPOT, Wildlife Computers) tags, and we deployed pop-up satellite archival transmitting (PSAT, model miniPAT, Wildlife Computers) tags, in addition to SPOT tags, on the individuals near Cape Cod $(\mathrm{n}=17$; Table $\mathrm{C}-1)$. Sharks were captured on rod and reel and brought onboard the fishing vessel where they were ventilated with a seawater hose and the hook was removed. SPOT tags were affixed to the dorsal fin using nylon bolts and contained a wet/dry switch that activated at the surface to transmit to the Argos satellite network from which a Doppler-based position was calculated. PSATs were tethered with a stainless steel wire to an intramuscular T-bar style spear tip $(\mathrm{n}=16)$ or a nylon umbrella dart $(\mathrm{n}=3)$. In situ measurements of pressure, temperature and light levels were collected every 15 seconds throughout the PSAT deployments and aggregated for satellite transmission. Archived depth and temperature data were transmitted as 4 summarized products: (1) time-at-temperature and (2) time-at-depth histograms for 12 bins every 24 hours; (3) depth-temperature profiles at 16 representative depths every 24 hours; (4) depth time series at 2.5 minute resolution. PSATs were programmed to detach after 180 days and transmit these summarized data products, along with daily light level data for geolocation, via the Argos satellite system.

Resulting SPOT-tag locations were processed with a Kalman filtering algorithm by Collecte Localisation Satellites (Lopez et al., 2014) and subsequently assigned error flags called location classes (LC): LC 3, <250 m; LC 2, 250-500 m; LC 1, 500-1500 m; LC 0, >1500 $\mathrm{m}$ for classes 3, 2, 1, 0. Additional classes A, B represent positions derived from less than 4 satellite messages which result in no estimates of spatial accuracy from CLS; however, recent work on several marine species and platforms suggests error for A, B classes is order $1-10 \mathrm{~km}$ and nearly always $<20 \mathrm{~km}$ (Lopez et al., 2014). Location class Z positions were considered invalid and removed from further analysis (CLS, 2016). Remaining positions were filtered using a speed filter $\left(4 \mathrm{~ms}^{-1}\right)$ from the trip package (Sumner et al., 2009) to remove unrealistic locations. The filtered Argos data were fit in a hierarchical fashion with a two-state switching state-space model (SSM) to estimate locations from the noisy Argos data, infer behavioral state and standardize the location time series (6 hr resolution) (Jonsen, 2016). The SSM combines a process model that estimates movement parameters and an observation model that accounts for spatial uncertainty using Markov Chain Monte Carlo (MCMC). The model inferred a behavior state based on fitted movement parameters (correlation, $\gamma$ and turn angle, $\theta$ ). Resident behavior (often referred to as area-restricted 
search or foraging) was characterized by $\theta$ near $180^{\circ}$ and $\gamma$ near 0 (short steps with large turn angles), while traveling (or transit) behavior produces movements in which $\theta$ is near $0^{\circ}$ and $\gamma$ near 1 (long, relatively straight tracks) between consecutive steps in the individual trajectories. Blue shark tracks were divided into trajectories for which data gaps were no longer than 4 days. Models were fit in JAGS (Plummer, 2004) using the bsam package (Jonsen, 2016) for R ( $\mathrm{R}$ Development Core Team, 2015). The models were fit with a 6 hour time step using two MCMC chains of 60,000 samples from which the first 40,000 were discarded as burn-in. Posterior inference was performed from the remaining 20,000 samples per chain after thinning by a factor of 20 to reduce within-chain sample autocorrelations, yielding a final 2,000 samples from the joint posterior. Model convergence was assessed using criteria outlined in (Jonsen, 2016) and included: posterior samples were stationary, MCMC chains were well-mixed, within-chain autocorrelation was low and the Brooks-Gelman-Rubin potential scale reduction factors $(\hat{r})$ were $\leq 1.1$.

\section{C.1.2 Oceanographic data}

To quantify associations between blue sharks and mesoscale eddies, we used the Mesoscale Eddy Trajectory Atlas distributed by Archiving Validation and Interpretation of Satellite and Oceanographic Data (AVISO; https://www.aviso.altimetry.fr/en/data/products/valueadded-products/global-mesoscale-eddy-trajectory-product.html) that describes daily tracks of coherent mesoscale structures (CMS) based on maps of surface altimetry (Chelton et al., 2011). Eddies with lifetimes greater than 4 weeks (28 days) are tracked based on their signatures in sea-level anomaly (SLA) fields. Prior to the identification and tracking of mesoscale eddies, the SLA fields are high-pass filtered using a $20^{\circ} \times 10^{\circ}$ (longitude $\mathrm{x}$ latitude) 2D weighted least-squares regression (LOESS) smoother to remove the effects of seasonal heating and cooling (Chelton et al., 2011) as:

$$
S S H=S L A-\langle S L A\rangle
$$

where the $<>$ operator indicates spatial smoothing. We developed a meander filter for the Gulf Stream region (see Section C.1.3) using daily, $0.25^{\circ}$ resolution absolute dynamic topography (ADT) data from AVISO which is generated by satellite-derived anomalies from the mean dynamic topography surface (Rio et al., 2011). Daily, $1 \mathrm{~km}$ sea surface temperature (SST) data was acquired from NASA JPL's Multi-scale Ultra-high Resolution (MUR) product. SRTM30+ bathymetry from Scripps was downloaded from NOAA CoastWatch server (ERDDAP id: srtm30plus) (Becker et al., 2009). 


\section{C.1.3 Eddy collocation}

Eddy occupation was quantified using a subset of the track data to include those positions that were in the Gulf Stream, a well-known region of mesoscale activity, in water deeper than $2000 \mathrm{~m}$ and that corresponded to the temporal limits of the eddy tracking dataset (Jan 1993 - Jan 2017). To focus our analysis on eddies, we developed a two-step meander filter for the Gulf Stream region (Gaube and McGillicuddy, 2017) in which we:

1. defined a mask $1^{\circ}$ north and $2^{\circ}$ south of the GS north wall (40 $\mathrm{cm}$ ADT contour). Those features in which the core of the CMS was within the meander mask were considered meanders and removed from the remainder of the analysis.

2. calculated net zonal displacement of eddies and removed those that exhibited primarily eastward displacement, following (Gaube and McGillicuddy, 2017), using a 3-day rolling window.

Shark locations were collocated to the nearest eddy identified in the eddy atlas (excluding meanders as described above) following (Gaube et al., 2017). We also used a maximum distance from the eddy center of $200 \mathrm{~km}$ or 2.5 times the length of the eddy radius to prevent collocation to eddies from an unreasonable distance to be biologically-relevant.

To assess differences in the distribution of blue sharks within anticyclonic and cyclonic eddies, we constructed histograms of blue shark location as a function of radial distance from the eddy center, resulting in number of shark positions per unit area of an annulus defined by the radial distance from the eddy center. To determine if individuals are more likely to be associated with the core, interior or periphery of eddies of either polarity, we defined eddy subregions by the normalized distance $r$ from the eddy SLA extremum, where the inner-core is defined as $r \leq L_{s} / 2$, the outer core as $L_{s} / 2<r \leq L_{s}$ and the eddy interior includes both the inner and outer core $r \leq L_{s}$. The eddy periphery is defined as $L_{s}<r \leq 2 L_{s}$ and the area outside of an eddy as $r>2 L_{s}$ (see Fig. 2 in Gaube et al., 2017).

We compared observed movements to two null models of eddy use by collocating simulated tracks and drifter data to the eddy field as described above. We generated 100 correlated random walk (CRW) simulations per blue shark using the distributions of turn angles and step lengths from each individual's observed movement data (adehabitatLT R package; Calenge, 2006). To match the spatial bias in presence data, CRW simulations were initiated at the tagging location for each individual and were constrained to realistic movements using bathymetry. CRW simulated tracks for each individual represent random eddy use based on chance of encountering these features. To assess the role of passive advection and the relative spatial composition of the sub-regions of eddies of each polarity, we collocated a surface drifter dataset from NOAA's Atlantic Oceanographic and Meteorological Laboratory (ftp://ftp.aoml.noaa.gov/pub/phod/buoydata/hourly_product/), using all 
drifters within the study region for a 5-year period (2005-2009). Significance testing of eddy use by tracked sharks was conducted by comparing the observed frequency of eddy use per individual to the confidence interval of eddy use by CRW simulations.

\section{C.1.4 Diving and vertical eddy structure}

PSAT tags were not programmed to transmit in situ temperature time series data. Thus, temperatures to accompany the depth time series were interpolated from daily depthtemperature profiles by computing a weighted least-squares regression of data using halfpower filter cutoffs of 5 days and $150 \mathrm{~m}$. The resulting depth-temperature time series of blue shark diving (from PSAT tags) was collocated to eddies at 6-hour intervals to match the temporal resolution of the standardized position data (from the SPOT tags). Individual dives in eddies were extracted from the time series data using the diveMove package (Luque, 2007 ) in R. Dives were characterized by movements below $200 \mathrm{~m}$ from shallower than $50 \mathrm{~m}$ for longer than 30 minutes and less than 6 hours. Individual dives in eddies were further characterized by their depth anomaly as:

$$
D^{\prime}(x, y, t)=D(x, y, t)-D_{12}(x, y, t)
$$

where $D^{\prime}$ is the depth anomaly of each dive, $D$ is the max depth of each dive, and $D_{12}$ is the climatological mean depth of the $12^{\circ} \mathrm{C}$ isotherm from the World Ocean Atlas. This metric indicates when eddies modulate shark dive depth by facilitating dives shallower $\left(D^{\prime}<0\right)$ or deeper $\left(D^{\prime}>0\right)$ than the climatological $12^{\circ} \mathrm{C}$ isotherm. Eddy modulation of isotherm depth was further analyzed by comparing HYCOM-modeled in situ depth of the $12^{\circ} \mathrm{C}$ isotherm within eddies with the climatological mean.

Eddy vertical composites were computed from the HYbrid Coordinate Ocean Model (HYCOM)(Chassignet et al., 2007) using modeled depth-temperature profiles for the eddies occupied by the sharks during the periods of occupation. Profiles were interpolated to $5 \mathrm{~m}$ intervals and summarized by calculating the mean profile at intervals of $L_{s} / 10$ from $-2 L_{s}$

to $2 L_{s}$. Temperature anomaly for the shark time series and eddy vertical composites were calculated by subtracting the climatological mean temperature from the World Ocean Atlas 2013 (Locarnini et al., 2013) at each depth level.

\section{C.2 Supplemental Tables}


Table C-1: Tagging summary for SPOT and PSAT-tagged blue sharks in this study. SPOT TAL indicates time-at-liberty (in days) of the Argosbased SPOT tag data and SPOT per Day the average number of SPOT positions per day over the deployment. PSAT TAL indicates time-at-liberty for the PSAT tag. Track distance is cumulative trajectory distance (in $\mathrm{km}$ ).

\begin{tabular}{llllllllll}
\hline Shark ID & $\begin{array}{l}\text { Tag } \\
\text { Type }\end{array}$ & Tag Date & $\begin{array}{l}\text { Tag } \\
\text { Lat }\left({ }^{\circ} \mathrm{N}\right)\end{array}$ & $\begin{array}{l}\text { Tag } \\
\text { Lon }\left({ }^{\circ} \mathrm{W}\right)\end{array}$ & $\begin{array}{l}\text { FL } \\
(\mathrm{cm})\end{array}$ & $\begin{array}{l}\text { SPOT } \\
\text { TAL }\end{array}$ & $\begin{array}{l}\text { SPOT } \\
\text { per } \\
\text { Day }\end{array}$ & $\begin{array}{l}\text { PSAT } \\
\text { TAL }\end{array}$ & $\begin{array}{l}\text { Distance } \\
(\mathrm{km})\end{array}$ \\
\hline 106744 & SP & $2016-08-27$ & 41.40 & 69.30 & 277 & 132 & 3.3 & 180 & 5,676 \\
106745 & SP & $2016-08-28$ & 41.52 & 69.43 & 270 & 131 & 4.5 & 180 & 5,587 \\
106746 & SP & $2016-08-28$ & 41.54 & 69.42 & 262 & 127 & 3.3 & 180 & 3,531 \\
106747 & SP & $2016-10-18$ & 41.47 & 69.33 & 295 & 74 & 4.3 & 172 & 3,501 \\
106748 & SP & $2016-08-27$ & 41.09 & 69.38 & 245 & 78 & 1.7 & DNR & 2,589 \\
132346 & S & $2013-07-28$ & 40.65 & 71.71 & 274 & 207 & 4.6 & NA & 8,205 \\
141195 & S & $2014-07-12$ & 40.79 & 71.37 & 295 & 64 & 6.3 & NA & 2,280 \\
141261 & SP & $2015-10-13$ & 41.32 & 69.28 & 254 & 259 & 3.4 & 180 & 12,428 \\
141262 & SP & $2016-08-28$ & 41.54 & 69.42 & 221 & 131 & 3.0 & 121 & 5,686 \\
141263 & SP & $2016-08-25$ & 41.49 & 69.33 & 248 & 70 & 2.8 & 39 & 3,083 \\
141264 & SP & $2015-10-21$ & 41.59 & 69.45 & 254 & 158 & 4.8 & DNR & 6,253 \\
141265 & SP & $2016-08-27$ & 41.09 & 69.38 & 241 & 67 & 3.1 & 16 & 2,536 \\
141266 & SP & $2016-09-10$ & 41.75 & 69.83 & 254 & 77 & 3.4 & 112 & 4,221 \\
141268 & SP & $2015-10-13$ & 41.58 & 69.42 & 267 & 120 & 5.6 & 134 & 5,731 \\
141270 & SP & $2015-10-21$ & 41.60 & 69.44 & 274 & 288 & 6.7 & 107 & 14,485 \\
165927 & SP & $2016-10-18$ & 41.47 & 69.33 & 313 & 80 & 4.7 & DNR & 3,885 \\
165928 & SP & $2016-10-18$ & 41.47 & 69.33 & 290 & 54 & 3.8 & 127 & 3,034 \\
\hline
\end{tabular}


Table C-2: Summary of anticyclonic (ACE) and cyclonic (CE) eddy use by blue sharks in the Gulf Stream. Values represent frequency (Freq) and number (N) of positions in eddies of each polarity relative to total number of SPOT positions within the study area for each individual. Correlated random walk $(\mathrm{CRW})$ values show frequency of eddy use by simulated random movements as mean (confidence interval). Asterisks $\left({ }^{*}\right)$ and crosses $\left({ }^{\dagger}\right)$ indicate the observed eddy use by sharks is greater than and less than the confidence interval calculated from CRW simulations, respectively. Final row gives overall averages across individuals.

\begin{tabular}{ccccccc}
\hline \multicolumn{4}{c}{ Anticyclonic Eddies } & \multicolumn{3}{c}{ Cyclonic Eddies } \\
Tag ID & Freq & $\mathrm{N}$ & CRW & Freq & N & CRW \\
\hline 106744 & $0.24^{*}$ & 93 & $0.17(0.15-0.19)$ & $0.28^{*}$ & 108 & $0.23(0.20-0.25)$ \\
106745 & $0.19^{*}$ & 68 & $0.13(0.12-0.15)$ & & 0 & $0.20(0.17-0.22)$ \\
106746 & & 0 & $0.18(0.15-0.22)$ & $0.28^{*}$ & 33 & $0.11(0.09-0.13)$ \\
106747 & & 1 & $0.14(0.12-0.16)$ & $0.07^{\dagger}$ & 18 & $0.18(0.16-0.21)$ \\
106748 & $0.33^{*}$ & 36 & $0.15(0.13-0.18)$ & & 0 & $0.19(0.15-0.22)$ \\
132346 & 0.20 & 21 & $0.20(0.17-0.22)$ & $0.14^{\dagger}$ & 15 & $0.19(0.17-0.21)$ \\
141195 & $0.12^{\dagger}$ & 20 & $0.15(0.13-0.18)$ & & 0 & $0.15(0.13-0.18)$ \\
141261 & 0.21 & 160 & $0.23(0.21-0.25)$ & $0.40^{*}$ & 308 & $0.28(0.26-0.3)$ \\
141262 & $0.20^{*}$ & 93 & $0.14(0.12-0.15)$ & $0.12^{\dagger}$ & 55 & $0.21(0.19-0.24)$ \\
141263 & $0.39^{*}$ & 63 & $0.15(0.12-0.18)$ & 0.17 & 28 & $0.17(0.14-0.19)$ \\
141264 & $0.37^{*}$ & 207 & $0.24(0.21-0.26)$ & $0.24^{*}$ & 138 & $0.21(0.19-0.23)$ \\
141265 & $0.10^{\dagger}$ & 16 & $0.15(0.11-0.18)$ & $0.04^{\dagger}$ & 7 & $0.17(0.14-0.19)$ \\
141266 & & 3 & $0.14(0.12-0.16)$ & 0.20 & 18 & $0.21(0.18-0.24)$ \\
141268 & $0.28^{*}$ & 45 & $0.24(0.21-0.26)$ & 0.18 & 30 & $0.18(0.16-0.21)$ \\
141270 & 0.20 & 212 & $0.21(0.19-0.23)$ & $0.25^{\dagger}$ & 259 & $0.28(0.27-0.3)$ \\
165927 & 0.02 & 6 & $0.16(0.14-0.19)$ & $0.14^{\dagger}$ & 35 & $0.23(0.20-0.26)$ \\
165928 & 0.15 & 23 & $0.16(0.14-0.19)$ & $0.08^{\dagger}$ & 12 & $0.16(0.12-0.20)$ \\
ALL & $0.19^{*}$ & 67 & $0.18(0.17-0.18)$ & $0.19^{\dagger}$ & 76 & $0.20(0.20-0.21)$ \\
\hline
\end{tabular}




\section{C.3 Supplemental Figures}
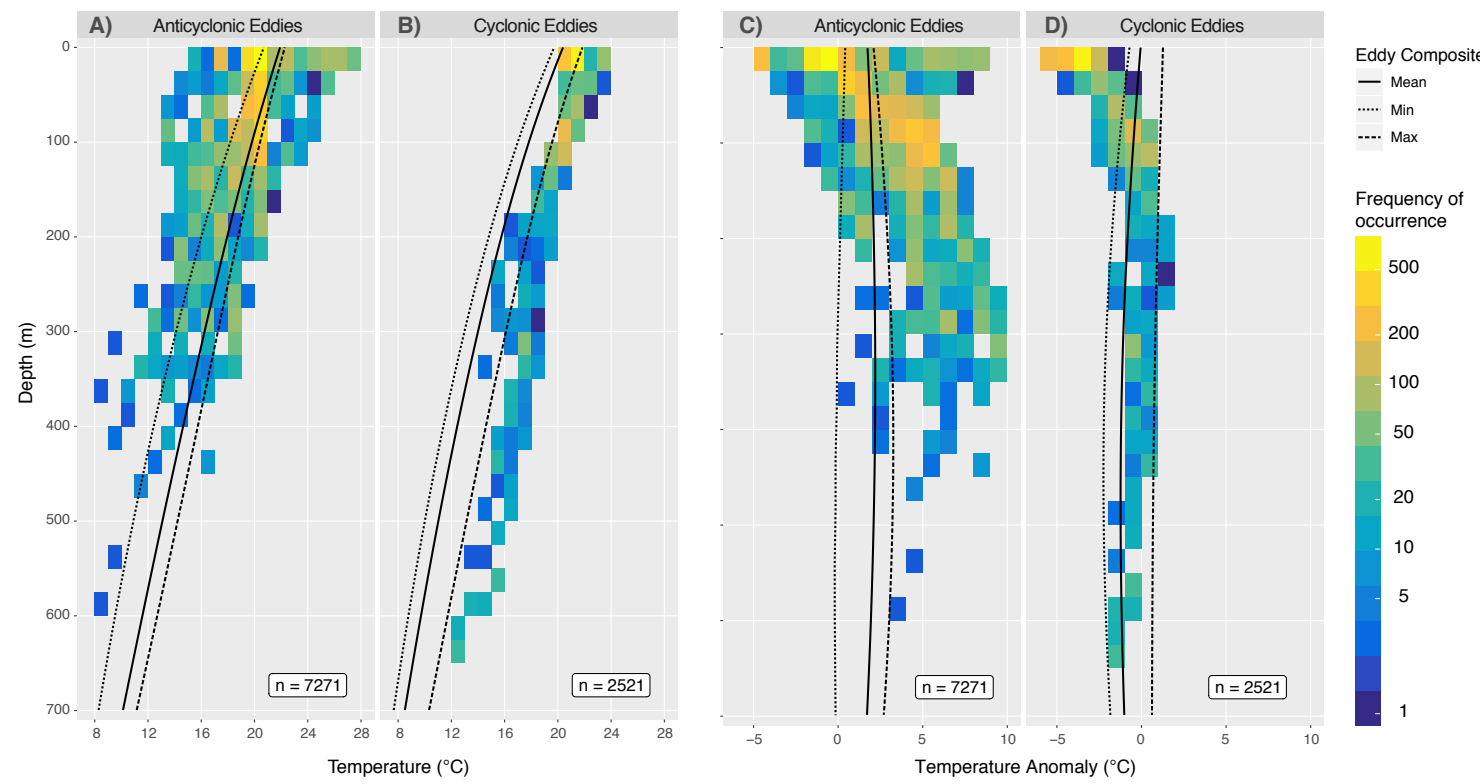

Figure C-1: Frequency of depth-temperature profile measurements recorded by PSAT-tagged blue sharks summarized into $1^{\circ}$ and $25 \mathrm{~m}$ bins. Color (log scale) denotes frequency of depth-temperature measurements within a given bin and eddy type for tag-measured temperature (A,B) and temperature anomaly of tag temperature as compared to World Ocean Atlas 2013 (C,D). Lines show mean and range of depth-temperature values from the HYCOM-derived eddy composites (see Figs. 5-2, C-2). Tag-measured depth-temperature data is from inner-core $\left(r \leq L_{s} / 2\right)$ of eddies only. 

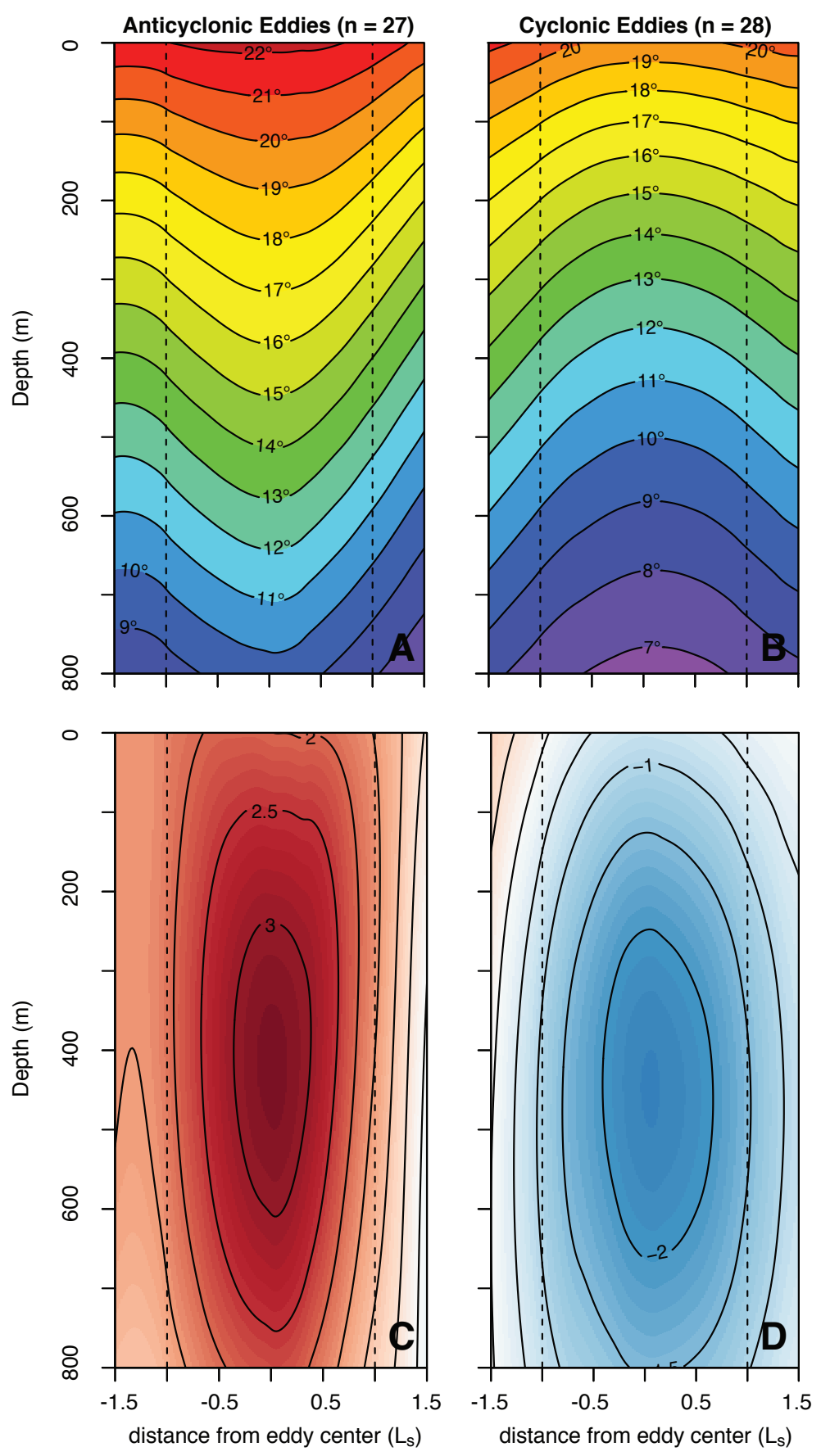

Figure C-2: Depth-temperature composite for Gulf Stream anticyclones (A) and cyclones (B) derived from HYCOM-modeled temperature fields and anticyclone (D) and cyclone (E) composite temperature anomalies. Dashed vertical lines indicate eddy core and horizontal labeled lines indicate isotherms. 
THIS PAGE INTENTIONALLY LEFT BLANK 


\section{BIBLIOGRAPHY}

Aarestrup, K., F. Okland, M. M. Hansen, D. Righton, P. Gargan, M. Castonguay, L. Bernatchez, P. Howey, H. Sparholt, M. I. Pedersen and R. S. McKinley, 2009. Oceanic Spawning Migration of the European Eel (Anguilla anguilla). Science 325(5948): 16601660. doi:10.1126/science.1178120.

Abascal, F. J., J. Mejuto, M. Quintans, B. Garcia-Cortes and A. Ramos-Cartelle, 2015. Tracking of the broadbill swordfish, Xiphias gladius, in the central and eastern North Atlantic. Fisheries Research 162(February): 20-28. doi:10.1016/j.fishres.2014.09.011.

Abascal, F. J., J. Mejuto, M. Quintans and A. Ramos-Cartelle, 2010. Horizontal and vertical movements of swordfish in the Southeast Pacific. Ices Journal of Marine Science 67(3): 466-474. doi:10.1093/Icesjms/Fsp252.

Abecassis, M., H. Dewar, D. Hawn and J. Polovina, 2012. Modeling swordfish daytime vertical habitat in the North Pacific Ocean from pop-up archival tags. Marine Ecology Progress Series 452: 219-236. doi:10.3354/meps09583.

Alvarado Bremer, J. R., J. Mejuto, J. Gómez-Márquez, F. Boán, P. Carpintero, J. M. Rodríguez, J. Viñas, T. W. Greig and B. Ely, 2005. Hierarchical analyses of genetic variation of samples from breeding and feeding grounds confirm the genetic partitioning of northwest Atlantic and South Atlantic populations of swordfish (Xiphias gladius L.). Journal of Experimental Marine Biology and Ecology 327(2): 167-182. doi:10.1016/j. jembe.2005.06.022.

Amante, C. and B. W. Eakins, 2009. ETOPO1 1 Arc-Minute Global Relief Model: Procedures, Data Sources and Analysis. NOAA Technical Memorandum NESDIS NGDC-24 page 19.

Arocha, F. and D. W. Lee, 1995. Maturity at size, reproductive seasonality, spawning frequency, fecundity and sex ratio in swordfish from the Northwest Atlantic. Collective Volume of Scientific Papers ICCAT 45: 350-357.

Backus, R. H. and J. E. Craddock, 1982. Mesopelagic fishes in Gulf Stream cold-core rings. Journal of Marine Research (USA) .

Bailleul, F., C. Cotté and C. Guinet, 2010. Mesoscale eddies as foraging area of a deepdiving predator, the southern elephant seal. Marine Ecology Progress Series 408: 251-264. doi:10.3354/meps08560.

Bakun, A., 2006. Fronts and eddies as key structures in the habitat of marine fish larvae: opportunity, adaptive response and competitive advantage. Scientia Marina 70(S2): 105122 .

Banzon, V., T. M. Smith, T. Mike Chin, C. Liu and W. Hankins, 2016. A long-term record of blended satellite and in situ sea-surface temperature for climate monitoring, 
modeling and environmental studies. Earth System Science Data 8(1): 165-176. doi: 10.5194/essd-8-165-2016.

Barthelme, S., 2016. imager: Image Processing Library Based on 'CImg'. R package version 0.40 .2 .

Baum, J. K., R. A. Myers, D. G. Kehler, B. Worm, S. J. Harley and P. A. Doherty, 2003. Collapse and conservation of shark populations in the Northwest Atlantic. Science 299(5605): 389-392.

Becker, J. J., D. T. Sandwell, W. H. F. Smith, J. Braud, B. Binder, J. Depner, D. Fabre, J. Factor, S. Ingalls and S. H. Kim, 2009. Global bathymetry and elevation data at 30 arc seconds resolution: SRTM30_PLUS. Marine Geodesy 32(4): 355-371.

Belkin, I. M., G. L. Hunt, E. L. Hazen, J. E. Zamon, R. Schick, R. Prieto, J. Brodziak, J. Hare, S. L. Teo, L. Thorne, H. Bailey, S. Itoh, P. Munk, M. K. Musyl, J. K. Willis and W. Zhang, 2014. Fronts, Fish, and Predators. Deep Sea Research Part II: Topical Studies in Oceanography 107: 1-2. doi:10.1016/j.dsr2.2014.07.009.

Belkin, I. M. and J. E. O'Reilly, 2009. An algorithm for oceanic front detection in chlorophyll and SST satellite imagery. Journal of Marine Systems 78(3): 319-326. doi:10.1016/j. jmarsys.2008.11.018.

Benitez-Nelson, C. R., R. R. Bidigare, T. D. Dickey, M. R. Landry, C. L. Leonard, S. L. Brown, F. Nencioli, Y. M. Rii, K. Maiti, J. W. Becker and Others, 2007. Mesoscale eddies drive increased silica export in the subtropical Pacific Ocean. Science 316(5827): $1017-1021$.

Berumen, M. L., C. D. Braun, J. E. M. Cochran, G. B. Skomal and S. R. Thorrold, 2014. Movement patterns of juvenile whale sharks tagged at an aggregation site in the red sea. PloS one 9(7): e103536. doi:10.1371/journal.pone.0103536.

Bianchi, D., E. D. Galbraith, D. A. Carozza, K. A. S. Mislan and C. A. Stock, 2013. Intensification of open-ocean oxygen depletion by vertically migrating animals. Nature Geoscience 6(7): 545-548. doi:10.1038/ngeo1837.

Bigelow, K. A., C. H. Boggs and X. I. He, 1999. Environmental effects on swordfish and blue shark catch rates in the US North Pacific longline fishery. Fisheries Oceanography 8(3): 178-198.

Bigelow, K. a. and M. N. Maunder, 2007. Does habitat or depth influence catch rates of pelagic species? Canadian Journal of Fisheries and Aquatic Sciences 64(11): 1581-1594. doi:10.1139/f07-115.

Bleck, R., 2002. An oceanic general circulation model framed in hybrid isopycnic-Cartesian coordinates. Ocean Modelling 4(1): 55-88. doi:10.1016/S1463-5003(01)00012-9.

Block, B. A., I. D. Jonsen, S. J. Jorgensen, A. J. Winship, S. A. Shaffer, S. J. Bograd, E. L. Hazen, D. G. Foley, G. A. Breed and A. L. Harrison, 2011. Tracking apex marine predator movements in a dynamic ocean. Nature 475(7354): 86-90. 
Block, B. A., S. L. H. Teo, A. Walli, A. Boustany, M. J. W. Stokesbury, C. J. Farwell, K. C. Weng, H. Dewar and T. D. Williams, 2005. Electronic tagging and population structure of Atlantic bluefin tuna. Nature 434(7037): 1121-1127.

Bonfil, R., 2005. Transoceanic Migration, Spatial Dynamics, and Population Linkages of White Sharks. Science 310(5745): 100-103. doi:10.1126/science.1114898.

Bost, C.-A., C. Cotté, F. Bailleul, Y. Cherel, J.-B. Charrassin, C. Guinet, D. G. Ainley and H. Weimerskirch, 2009. The importance of oceanographic fronts to marine birds and mammals of the southern oceans. Journal of Marine Systems 78(3): 363-376.

Braun, C. D., B. Galuardi and S. R. Thorrold, 2018a. HMMoce: An R package for improved geolocation of archival-tagged fishes using a hidden Markov method. Methods in Ecology and Evolution 9: 1212-1220. doi:10.1111/2041-210X.12959.

Braun, C. D., M. B. Kaplan, A. Z. Horodysky and J. K. Llopiz, 2015a. Satellite telemetry reveals physical processes driving billfish behavior. Animal Biotelemetry 3(1): 2. doi: 10.1186/s40317-014-0020-9.

Braun, C. D., G. B. Skomal and S. R. Thorrold, 2018b. Integrating archival tag data and a high-resolution oceanographic model to estimate basking shark (Cetorhinus maximus) movements in the western Atlantic. Frontiers in Marine Science 5(25). doi:10.3389/fmars. 2018.00025 .

Braun, C. D., G. B. Skomal, S. R. Thorrold and M. L. Berumen, 2014. Diving Behavior of the Reef Manta Ray Links Coral Reefs with Adjacent Deep Pelagic Habitats. PLoS One 9(2): e88170. doi:10.1371/journal.pone.0088170.

Braun, C. D., G. B. Skomal, S. R. Thorrold and M. L. Berumen, 2015b. Movements of the reef manta ray (Manta alfredi) in the Red Sea using satellite and acoustic telemetry. Marine Biology 162(12): 2351-2362.

Bremer, J. R. A., J. Mejuto, T. W. Greig and B. Ely, 1996. Global population structure of the swordfish (Xiphias gladius L.) as revealed by analysis of the mitochondrial DNA control region. Journal of Experimental Marine Biology and Ecology 197(2): 295-310.

Brodie, S., M. G. Jacox, S. J. Bograd, H. Welch, H. Dewar, K. L. Scales, S. M. Maxwell, D. K. Briscoe, C. A. Edwards, L. B. Crowder, R. L. Lewison and E. L. Hazen, 2018. Integrating dynamic subsurface habitat metrics into species distribution models. Frontiers in Marine Science 5(June): 219. doi:10.3389/FMARS.2018.00219.

Brunnschweiler, J. M., H. Baensch, S. J. Pierce and D. W. Sims, 2009. Deep-diving behaviour of a whale shark Rhincodon typus during long-distance movement in the western Indian Ocean. J Fish Biol 74(3): 706-714. doi:10.1111/J.1095-8649.2008.02155.X.

Byrne, M. E., E. Cortés, J. J. Vaudo, G. C. M. Harvey, M. Sampson, B. M. Wetherbee and M. Shivji, 2017. Satellite telemetry reveals higher fishing mortality rates than previously estimated, suggesting overfishing of an apex marine predator. Proceedings of the Royal Society B: Biological Sciences 284(1860): 20170658. doi:10.1098/rspb.2017.0658.

Calabrese, J. M., C. H. Fleming, E. Gurarie and R. Freckleton, 2016. Ctmm: an R Package for Analyzing Animal Relocation Data As a Continuous-Time Stochastic Process. Methods in Ecology and Evolution 7(9): 1124-1132. doi:10.1111/2041-210X.12559. 
Calenge, C., 2006. The package "adehabitat" for the R software: A tool for the analysis of space and habitat use by animals. Ecological Modelling 197(3-4): 516-519. doi:10.1016/ j.ecolmodel.2006.03.017.

Campana, S. E., A. Dorey, M. Fowler, W. Joyce, Z. Wang, D. Wright and I. Yashayaev, 2011. Migration Pathways, Behavioural Thermoregulation and Overwintering Grounds of Blue Sharks in the Northwest Atlantic. PLoS One 6(2): e16854. doi:10.1371/journal. pone.0016854.

Carey, F. G., 1982. A Brain Heater in the Swordfish. Science 216(4552): 1327-1329.

Carey, F. G., 1990. Further acoustic telemetry observations of swordfish. In Planning the Future of Billfishes, Research and Management in the 90s and Beyond, volume 13, pages 103-122. National Coalition for Marine Conservation, Inc.

Carey, F. G. and B. H. Robison, 1981. Daily patterns in the activities of swordfish, Xiphias gladius, observed by acoustic telemetry. Fishery Bulletin 79(2): 277-292.

Carey, F. G. and J. V. Scharold, 1990. Movements of blue sharks (Prionace glauca) in depth and course. Marine Biology 106(3): 329-342.

Chaprales, W., M. Lutcavage, R. Brill, B. Chase and G. Skomal, 1998. Harpoon Method for Attaching Ultrasonic and "Popup" Satellite Tags to Giant Bluefin Tuna and Large Pelagic Fishes. Marine Technology Society Journal 32(1): 104.

Chassignet, E. P., H. E. Hurlburt, O. M. Smedstad, G. R. Halliwell, P. J. Hogan, A. J. Wallcraft, R. Baraille and R. Bleck, 2007. The HYCOM (HYbrid Coordinate Ocean Model) data assimilative system. Journal of Marine Systems 65(1-4 SPEC. ISS.): 60-83. doi:10.1016/j.jmarsys.2005.09.016.

Chelton, D. B., M. G. Schlax and R. M. Samelson, 2011. Global observations of nonlinear mesoscale eddies. Progress in Oceanography 91(2): 167-216. doi:10.1016/j.pocean.2011. 01.002 .

CLS, 2016. Argos User Manual. Technical report, Collecte Localisation Satellites.

Collette, B. B., K. E. Carpenter, B. A. Polidoro, M. J. Juan-Jordá, A. Boustany, D. J. Die, C. Elfes, W. Fox, J. Graves and L. R. Harrison, 2011. High value and long life-double jeopardy for tunas and billfishes. Science 333(6040): 291-292.

Compagno, L. J. V., 1984. FAO Species Catalogue. IV. Sharks of the World. 1. Hexanchiformes to Laminiformes. Technical report, Food and Agriculture Organisation of the United Nations, Rome.

Cullis-Suzuki, S. and D. Pauly, 2010. Failing the high seas: A global evaluation of regional fisheries management organizations. Marine Policy 34(5): 1036-1042. doi:10.1016/j. marpol.2010.03.002.

Curtis, T. H., S. I. Zeeman, E. L. Summers, S. X. Cadrin and G. B. Skomal, 2014. Eyes in the sky: linking satellite oceanography and biotelemetry to explore habitat selection by basking sharks. Animal Biotelemetry 2(1): 12. doi:10.1186/2050-3385-2-12. 
Dawe, E. G. and P. C. Beck, 1985. Distribution and size of juvenile short-finned squid (Illex illecebrosus)(Mollusca, Cephalopoda) South of Newfoundland during winter. Vie et Milieu (France) .

de Boyer Montégut, C., G. Madec, A. S. Fischer, A. Lazar and D. Iudicone, 2004. Mixed layer depth over the global ocean: An examination of profile data and a profile-based climatology. Journal of Geophysical Research: Oceans 109(C12).

Dewar, H., E. D. Prince, M. K. Musyl, R. W. Brill, C. Sepulveda, J. Luo, D. Foley, E. S. Orbesen, M. L. Domeier and N. Nasby-Lucas, 2011. Movements and behaviors of swordfish in the Atlantic and Pacific Oceans examined using popup satellite archival tags. Fisheries Oceanography 20(3): 219-241.

Doherty, P. D., J. M. Baxter, F. R. Gell, B. J. Godley, R. T. Graham, G. Hall, J. Hall, L. A. Hawkes, S. M. Henderson, L. Johnson, C. Speedie and M. J. Witt, 2017. Long-term satellite tracking reveals variable seasonal migration strategies of basking sharks in the north-east Atlantic. Scientific Reports 7(February): 42837. doi:10.1038/srep42837.

Domeier, M. L., 2006. An analysis of Pacific striped marlin (Tetrapturus audax) horizontal movement patterns using pop-up satellite archival tags. Bulletin of Marine Science 79(3): $811-825$.

Donaldson, M. R., S. G. Hinch, C. D. Suski, A. T. Fisk, M. R. Heupel and S. J. Cooke, 2014. Making connections in aquatic ecosystems with acoustic telemetry monitoring. Frontiers in Ecology and the Environment 12(10): 565-573.

Dufois, F., N. J. Hardman-Mountford, J. Greenwood, A. J. Richardson, M. Feng and R. J. Matear, 2016. Anticyclonic eddies are more productive than cyclonic eddies in subtropical gyres because of winter mixing. Science Advances 2(5): e1600282.

Dulvy, N. K., J. K. Baum, S. Clarke, L. J. V. Compagno, E. Cortés, A. Domingo, S. Fordham, S. Fowler, M. P. Francis, C. Gibson, J. Martínez, J. A. Musick, A. Soldo, J. D. Stevens and S. Valenti, 2008. You can swim but you can't hide: the global status and conservation of oceanic pelagic sharks and rays. Aquatic Conservation: Marine and Freshwater Ecosystems 18(5): 459-482. doi:10.1002/aqc.975.

Evans, K., F. Abascal, D. Kolody, T. Sippel, J. Holdsworth and P. Maru, 2014. The horizontal and vertical dynamics of swordfish in the South Pacific Ocean. Journal of Experimental Marine Biology and Ecology 450: 55-67. doi:10.1016/j.jembe.2013.10.025.

Everhart, W. H., 1975. Principles of fisheries science. Comstock.

FAO, 2012. Xiphias gladius - FAO Fisheries and Aquaculture.

Fedulov, T. P. and A. I. Arkhipkin, 1986. Distribution of mass species of pelagic squids in the spring between Nova Scotia and the Sargasso Sea as a function of hydrologic and dynamic water structure. Oceanology 26(2): 229-234.

Fennell, S. and G. Rose, 2015. Oceanographic influences on Deep Scattering Layers across the North Atlantic. Deep-Sea Research Part I: Oceanographic Research Papers 105: 132141. doi:10.1016/j.dsr.2015.09.002. 
Ferretti, F., B. Worm, G. L. Britten, M. R. Heithaus and H. K. Lotze, 2010. Patterns and ecosystem consequences of shark declines in the ocean. Ecol Lett 13(8): 1055-1071.

Flierl, G. R. and D. J. McGillicuddy, 2002. Mesoscale and submesoscale physical-biological interactions. In The Sea, Volume 12, Biological-Physical Interactions in the Sea, volume 12, pages 113-185. Wiley: New York.

Francis, M. and C. Duffy, 2002. Distribution, seasonal abundance and bycatch of basking sharks (Cetorhinus maximus) in New Zealand, with observations on their winter habitat. Marine Biology 140(4): 831-842.

Franks, P. J. S., 1992. Phytoplankton blooms at fronts: patterns, scales, and physical forcing mechanisms. Reviews in Aquatic Sciences 6(2): 121-137.

Franks, P. J. S., J. S. Wroblewski and G. R. Flierl, 1986. Prediction of phytoplankton growth in response to the frictional decay of a warm-core ring. Journal of Geophysical Research 91(C6): 7603-7610.

Fuglister, F. C., 1963. Gulf stream'60. Progress in Oceanography 1: 265-373.

Galarza, J. A., J. Carreras-Carbonell, E. Macpherson, M. Pascual, S. Roques, G. F. Turner and C. Rico, 2009. The influence of oceanographic fronts and early-life-history traits on connectivity among littoral fish species. Proceedings of the National Academy of Sciences 106(5): 1473-1478. doi:10.1073/pnas.0806804106.

Galuardi, B., 2017. boaR: Belkin O'Reilly front detection algorithm. R package version 0.1.

Galuardi, B. and C. H. Lam, 2014. Chapter nineteen - telemetry analysis of highly migratory species. In S. X. Cadrin, L. A. Kerr and S. Mariani, editors, Stock Identification Methods (Second Edition), pages 447 - 476. Academic Press, San Diego, second edition. doi: 10.1016/B978-0-12-397003-9.00019-9.

Galuardi, B. and M. Lutcavage, 2012. Dispersal Routes and Habitat Utilization of Juvenile Atlantic Bluefin Tuna, Thunnus thynnus, Tracked with Mini PSAT and Archival Tags. PLoS One 7(5). doi:10.1371/journal.pone.0037829.

Galuardi, B., A. Nielsen and M. Lutcavage, 2008. Optimizing smoothed sea surface temperature for improving archival tag geolocation. Marine Ecology Progress Series 365: 35-44. doi:10.3354/meps07497.

Galuardi, B., F. Royer, W. Golet, J. Logan, J. Neilson and M. Lutcavage, 2010. Complex migration routes of Atlantic bluefin tuna (Thunnus thynnus) question current population structure paradigm. Canadian Journal of Fisheries and Aquatic Sciences 67(6): 966-976. doi:10.1139/F10-033.

Game, E. T., H. S. Grantham, A. J. Hobday, R. L. Pressey, A. T. Lombard, L. E. Beckley, K. Gjerde, R. Bustamante, H. P. Possingham and A. J. Richardson, 2009. Pelagic protected areas: the missing dimension in ocean conservation. Trends in Ecology and Evolution 24(7): 360-369. doi:10.1016/j.tree.2009.01.011.

Garcia, H. E., R. A. Locarnini, T. P. Boyer, J. I. Antonov, O. K. Baranova, M. M. Zweng, J. R. Reagan and D. R. Johnson, 2014. World ocean atlas 2013, Volume 3: Dissolved Oxygen, Apparent Oxygen Utilization, and Oxygen Saturation . 
Garcia-Cortes, B. and M. Quintans, 2003. Summary of swordfish (xiphias gladius) recaptures carried out by the spanish surface longline fleet. Col.Vol.Sci.Pap. ICCAT 55(4): 14761484 .

Gaube, P., C. Barcelo, D. J. McGillicuddy Jr, A. Domingo, P. Miler, B. Giffoni, N. Marcovaldi and Y. Swimmer, 2017. The Use of Mesoscale Eddies by Juvenile Loggerhead Sea Turtles (Caretta caretta) in the Southwestern Atlantic. PLoS One 12(3): e0172839. doi:10.1371/journal.pone.0172839.

Gaube, P., C. D. Braun, G. L. Lawson, D. J. McGillicuddy, A. D. Penna, G. B. Skomal, C. Fischer and S. R. Thorrold, 2018. Mesoscale eddies influence the movements of mature female white sharks in the Gulf Stream and Sargasso Sea. Scientific Reports 8(1): 7363. doi:10.1038/s41598-018-25565-8.

Gaube, P. and D. J. McGillicuddy, 2017. The influence of Gulf Stream eddies and meanders on near-surface chlorophyll. Deep-Sea Research Part I: Oceanographic Research Papers 122: 1-16. doi:10.1016/j.dsr.2017.02.006.

Gaube, P., D. J. McGillicuddy Jr, D. B. Chelton, M. J. Behrenfeld and P. G. Strutton, 2014. Regional variations in the influence of mesoscale eddies on near-surface chlorophyll. Journal of Geophysical Research: Oceans 119: 1-26. doi:10.1002/2014JC010111.

Godø, O. R., A. Samuelsen, G. J. Macaulay, R. Patel, S. S. Hjøllo, J. Horne, S. Kaartvedt and J. A. Johannessen, 2012. Mesoscale eddies are oases for higher trophic marine life. PLoS One 7(1): e30161.

Gore, M. A., D. Rowat, J. Hall, F. R. Gell and R. F. Ormond, 2008. Transatlantic migration and deep mid-ocean diving by basking shark. Biology Letters 4(4): 395-398.

Group, T. R., 1981. Gulf Stream cold-core rings- Their physics, chemistry, and biology. Science 212: 1091-1100.

Haury, L. R., J. A. McGowan and P. H. Wiebe, 1978. Patterns and processes in the timespace scales of plankton distributions. In Spatial pattern in plankton communities, pages 277-327. Springer.

Hays, G. C., V. J. Hobson, J. D. Metcalfe, D. Righton and D. W. Sims, 2006. Flexible foraging movements of leatherback turtles across the North Atlantic ocean. Ecology 87(10): $2647-2656$.

Hazen, E. L., S. Jorgensen, R. R. Rykaczewski, S. J. Bograd, D. G. Foley, I. D. Jonsen, S. a. Shaffer, J. P. Dunne, D. P. Costa, L. B. Crowder and B. a. Block, 2012. Predicted habitat shifts of Pacific top predators in a changing climate. Nature Climate Change 3(3): 234-238. doi:10.1038/nclimate1686.

Hazen, E. L., K. L. Scales, S. M. Maxwell, D. K. Briscoe, H. Welch, S. J. Bograd, H. Bailey, S. R. Benson, T. Eguchi, H. Dewar, S. Kohin, D. P. Costa, L. B. Crowder and R. L. Lewison, 2018. A dynamic ocean management tool to reduce bycatch and support sustainable fisheries. Science Advances in press.

Hazin, H. and K. Erzini, 2008. Assessing swordfish distribution in the South Atlantic from spatial predictions. Fisheries Research 90(1-3): 45-55. doi:10.1016/j.fishres.2007.09.010. 
Hijmans, R. J., 2016. raster: Geographic Data Analysis and Modeling. R package version 2.6-7.

Hilborn, R., T. A. Branch, B. Ernst, A. Magnusson, C. V. Minte-Vera, M. D. Scheuerell and J. L. Valero, 2003. State of the World's Fisheries. Annual Review of Environment and Resources 28: 359-399. doi:10.1146/annurev.energy.28.050302.105509.

Hill, R. D., 1994. Theory of geolocation by light levels. Elephant seals: population ecology, behavior, and physiology. University of California Press, Berkeley pages 227-236.

Hill, R. D. and M. J. Braun, 2001. Geolocation by light level. In Electronic tagging and tracking in marine fisheries: Proceedings of the Symposium on Tagging and Tracking Marine Fish with Electronic Devices, pages 315-330. Springer, University of Hawaii.

Hinton, M. G. and H. Nakano, 1996. Standardizing catch and effort statistics using physiological, ecological, or behavioral constraints, and environmental data, with applications to blue marlin (Makaira nigricans) and swordfish (Xiphias gladius) of the Pacific Ocean. Ph.D. thesis, University of California, San Diego.

Hobday, A. J. and J. R. Hartog, 2014. Derived Ocean Features for Dynamic Ocean Management. Oceanography 27(4): 134-145. doi:10.5670/oceanog.2014.92.

Hoelzel, A. R., M. S. Shivji, J. Magnussen and M. P. Francis, 2006. Low worldwide genetic diversity in the basking shark (Cetorhinus maximus). Biology Letters 2(4): 639-642.

Hoffmayer, E. R., W. B. Driggers, J. S. Franks, D. S. Hanisko, M. a. Roffer and L. E. Cavitt, 2011. Recent occurrences of basking sharks, Cetorhinus maximus (Chondrichthyes: Cetorhinidae), in the Gulf of Mexico. Marine Biodiversity Records 4(March): e87. doi: $10.1017 /$ S1755267211000844.

Holzmann, H., A. Munk, M. Suster and W. Zucchini, 2006. Hidden Markov models for circular and linear-circular time series. Environmental and Ecological Statistics 13(3): $325-347$.

Hsu, A. C., A. M. Boustany, J. J. Roberts, J. H. Chang and P. N. Halpin, 2015. Tuna and swordfish catch in the U.S. northwest Atlantic longline fishery in relation to mesoscale eddies. Fisheries Oceanography 24(6): 508-520. doi:10.1111/fog.12125.

Hussey, N. E., S. T. Kessel, K. Aarestrup, S. J. Cooke, P. D. Cowley, A. T. Fisk, R. G. Harcourt, K. N. Holland, S. J. Iverson, J. F. Kocik, J. E. Mills Flemming and F. G. Whoriskey, 2015. Aquatic animal telemetry: A panoramic window into the underwater world. Science 348(6240). doi:10.1126/science.1255642.

Irigoien, X., T. A. Klevjer, A. Røstad, U. Martinez, G. Boyra, J. L. Acuña, A. Bode, F. Echevarria, J. I. Gonzalez-Gordillo, S. Hernandez-Leon, S. Agusti, D. L. Aksnes, C. M. Duarte and S. Kaartvedt, 2014. Large mesopelagic fishes biomass and trophic efficiency in the open ocean. Nat Commun 5. doi:10.1038/ncomms4271.

Jackson, J. B. C., M. X. Kirby, W. H. Berger, K. A. Bjorndal, L. W. Botsford, B. J. Bourque, R. H. Bradbury, R. Cooke, J. Erlandson, J. A. Estes, T. P. Hughes, S. Kidwell, C. B. Lange, H. S. Lenihan, J. M. Pandolfi, C. H. Peterson, R. S. Steneck, M. J. Tegner and R. R. Warner, 2001. Historical overfishing and the recent collapse of coastal ecosystems. Science 293(5530): 629-37. doi:10.1126/science.1059199. 
Johnson, D. S., J. M. London, M. A. Lea and J. W. Durban, 2008. Continuous-time correlated random walk model for animal telemetry data. Ecology 89(5): 1208-1215.

Johnston, D. W. and A. J. Read, 2007. Flow-field observations of a tidally driven island wake used by marine mammals in the Bay of Fundy, Canada. Fisheries Oceanography 16(5): 422-435.

Jonsen, I., 2016. Joint estimation over multiple individuals improves behavioural state inference from animal movement data. Scientific Reports (October 2015): 1-9. doi: $10.1038 /$ srep20625.

Jonsen, I., M. Basson, S. Bestley, M. Bravington, T. Patterson, M. Pedersen, R. Thomson, U. Thygesen and S. Wotherspoon, 2013. State-space models for bio-loggers: A methodological road map. Deep Sea Research Part II: Topical Studies in Oceanography 88-89: 34-46. doi:10.1016/j.dsr2.2012.07.008.

Jonsen, I. D., J. M. Flemmings and R. a. Myers, 2005. Robust State-Space Modeling of Animal Movement Data. Ecology 86(11): 2874-2880. doi:10.1890/04-1852.

Jorgensen, S. J., N. S. Arnoldi, E. E. Estess, T. K. Chapple, M. Rückert, S. D. Anderson and B. A. Block, 2012. Eating or Meeting? Cluster Analysis Reveals Intricacies of White Shark (Carcharodon carcharias) Migration and Offshore Behavior. PLoS One 7(10): e47819.

Josse, E., P. Bach and L. Dagorn, 1998. Simultaneous observations of tuna movements and their prey by sonic tracking and acoustic surveys. Hydrobiologia 371: 61-69.

Joyce, T. M., 1985. Gulf Stream warm-core ring collection: An introduction. Journal of Geophysical Research: Oceans 90(C5): 8801-8802.

Kobayashi, D. R., I.-J. Cheng, D. M. Parker, J. J. Polovina, N. Kamezaki and G. H. Balazs, 2011. Loggerhead turtle (Caretta caretta) movement off the coast of Taiwan: characterization of a hotspot in the East China Sea and investigation of mesoscale eddies. ICES Journal of Marine Science 68(4): 707-718.

Kohler, N. E., 1988. Aspects of the feeding ecology of the blue shark (Prionace glauca) in the Western North Atlantic. Ph.D. thesis, University of Rhode Island.

Kohler, N. E. and P. A. Turner, 2001. Shark tagging: A review of conventional methods and studies. Environmental Biology of Fishes 60(1-3): 191-223.

Lam, C. H., B. Galuardi and M. E. Lutcavage, 2014. Movements and oceanographic associations of bigeye tuna (Thunnus obesus) in the Northwest Atlantic. Canadian Journal of Fisheries and Aquatic Sciences 1543(September 2013): 1-15. doi:10.1139/cjfas-2013-0511.

Lam, C. H., B. Galuardi, A. Mendillo, E. Chandler and M. E. Lutcavage, 2016. Sailfish migrations connect productive coastal areas in the West Atlantic Ocean. Scientific Reports 6(August): 38163. doi:10.1038/srep38163.

Lam, C. H., A. Nielsen and J. R. Sibert, 2008. Improving light and temperature based geolocation by unscented Kalman filtering. Fisheries Research 91: 15-25. doi:10.1016/j. fishres.2007.11.002. 
Lam, C. H., A. Nielsen and J. R. Sibert, 2010. Incorporating sea-surface temperature to the light-based geolocation model TrackIt. Marine Ecology Progress Series 419: 71-84. doi:10.3354/meps08862.

Lavery, T. J., B. Roudnew, P. Gill, J. Seymour, L. Seuront, G. Johnson, J. G. Mitchell and V. Smetacek, 2010. Iron defecation by sperm whales stimulates carbon export in the Southern Ocean. Proceedings of the Royal Society B: Biological Sciences 277(1699): 3527-3531. doi:10.1098/rspb.2010.0863.

Lawson, G. L., M. R. Castleton and B. A. Block, 2010. Movements and diving behavior of Atlantic bluefin tuna Thunnus thynnus in relation to water column structure in the northwestern Atlantic. Marine Ecology Progress Series 400: 245.

Le Bris, A., A. Fréchet and J. S. Wroblewski, 2013. Supplementing electronic tagging with conventional tagging to redesign fishery closed areas. Fisheries Research 148(January 2016): 106-116. doi:10.1016/j.fishres.2013.08.013.

Le Fèvre, J., 1987. Aspects of the Biology of Frontal Systems. In Advances in Marine Biology, volume 23, pages 163-299. Academic Press. doi:10.1016/S0065-2881(08)60109-1.

Lerner, J. D., D. W. Kerstetter, E. D. Prince, L. Talaue-McManus, E. S. Orbesen, A. Mariano, D. Snodgrass and G. L. Thomas, 2013. Swordfish Vertical Distribution and Habitat Use in Relation to Diel and Lunar Cycles in the Western North Atlantic. Transactions of the American Fisheries Society 142(1): 95-104.

Lévy, M., R. Ferrari, P. J. S. Franks, A. P. Martin and P. Rivière, 2012. Bringing Physics to Life at the Submesoscale. Geophysical Research Letters 39(14): L14602.

Lewison, R., A. J. Hobday, S. Maxwell, E. Hazen, J. R. Hartog, D. C. Dunn, D. Briscoe, S. Fossette, C. E. O'keefe and M. Barnes, 2015. Dynamic ocean management: identifying the critical ingredients of dynamic approaches to ocean resource management. BioScience 65(5): 486-498.

Locarnini, R. A., A. V. Mishonov, J. I. Antonov, T. P. Boyer, H. E. Garcia, O. K. Baranova, M. M. Zweng, C. R. Paver, J. R. Reagan and D. R. Johnson, 2013. World Ocean Atlas 2013, Volume 1: Temperature. NOAA Atlas NESDIS 73: 40.

Loefer, J. K., G. R. Sedberry and J. C. McGovern, 2007. Nocturnal depth distribution of western North Atlantic swordfish (Xiphias gladius, Linnaeus, 1758) in relation to lunar illumination. Gulf and Caribbean Research 19(2): 83-88.

Lopez, R., J.-P. Malarde, F. Royer and P. Gaspar, 2014. Improving Argos doppler location using multiple-model Kalman filtering. IEEE Transactions on Geoscience and Remote Sensing 52(8): 4744-4755.

Luo, J., J. S. Ault, L. K. Shay, J. P. Hoolihan, E. D. Prince, C. a. Brown and J. R. Rooker, 2015. Ocean Heat Content Reveals Secrets of Fish Migrations. Plos One 10(10): e0141101. doi:10.1371/journal.pone.0141101.

Luque, S. P., 2007. Diving behaviour analysis in R. $R$ news 7(3): 8-14.

Mahadevan, A., 2014. Eddy effects on biogeochemistry. Nature 506: 168-169. doi:10.1038/ nature13048. 
Mahadevan, A., 2016. The Impact of Submesoscale Physics on Primary Productivity of Plankton. Annual Review of Marine Science 8(1): 161-184. doi:10.1146/ annurev-marine-010814-015912.

Mann, K. H. and J. R. N. Lazier, 2006. Dynamics of Marine Ecosystems. Oceanography 19(2): 157.

Marchal, E., F. Gerlotto and B. Stéquert, 1993. On the relationship between scattering layer, thermal structure and tuna abundance in the eastern Atlantic equatorial current system. Oceanologica Acta 16(3): 261-272.

Maunder, M. N. and A. E. Punt, 2004. Standardizing catch and effort data: a review of recent approaches. Fisheries Research 70(2): 141-159. doi:10.1016/j.fishres.2004.08.002.

Maxwell, S. M., E. L. Hazen, R. L. Lewison, D. C. Dunn, H. Bailey, S. J. Bograd, D. K. Briscoe, S. Fossette, A. J. Hobday and M. Bennett, 2015. Dynamic ocean management: Defining and conceptualizing real-time management of the ocean. Marine Policy 58: $42-50$.

McGillicuddy, D., L. Anderson and N. Bates, 2007. Eddy / Wind Interactions Stimulate Extraordinary Mid-Ocean Plankton Blooms. Science 316: 1021-1026.

McGillicuddy, D. J., 2016. Mechanisms of Physical-Biological-Biogeochemical Interaction at the Oceanic Mesoscale. Annual Review of Marine Science 8(1): 125-159. doi:10.1146/ annurev-marine-010814-015606.

Metcalfe, J. D. and G. P. Arnold, 1997. Tracking fish with electronic tags. Nature 387(6634): 665.

Michelot, T., R. Langrock and T. A. Patterson, 2016. moveHMM: An R package for the statistical modelling of animal movement data using hidden Markov models. Methods in Ecology and Evolution 7(11): 1308-1315.

Miller, C. B. and P. A. Wheeler, 2012. Biological oceanography. John Wiley \& Sons.

Miller, P. I., K. L. Scales, S. N. Ingram, E. J. Southall and D. W. Sims, 2015. Basking sharks and oceanographic fronts: Quantifying associations in the north-east Atlantic. Functional Ecology 29(8): 1099-1109. doi:10.1111/1365-2435.12423.

Mourato, B. L., F. Hazin, K. Bigelow, M. Musyl, F. Carvalho and H. Hazin, 2014. Spatiotemporal trends of sailfish, Istiophorus platypterus catch rates in relation to spawning ground and environmental factors in the equatorial and southwestern Atlantic Ocean. Fisheries Oceanography 23(1): 32-44. doi:10.1111/fog.12040.

Musyl, M. K., M. L. Domeier, N. Nasby-Lucas, R. W. Brill, L. M. McNaughton, J. Y. Swimmer, M. S. Lutcavage, S. G. Wilson, B. Galuardi and J. B. Liddle, 2011. Performance of pop-up satellite archival tags. Marine Ecology Progress Series doi:10.3354/meps09202.

Myers, R. a. and B. Worm, 2005. Extinction, survival or recovery of large predatory fishes. Philosophical transactions of the Royal Society of London. Series B, Biological sciences 360(1453): 13-20. doi:10.1098/rstb.2004.1573. 
Nakano, H. and J. D. Stevens, 2008. The Biology and Ecology of the Blue Shark, Prionace Glauca. In Sharks of the Open Ocean, pages 140-151. Blackwell Publishing Ltd. doi: 10.1002/9781444302516.ch12.

Neilson, J., F. Arocha, S. Cass-Calay, J. Mejuto, M. Ortiz, G. Scott, C. Smith, P. Travassos, G. Tserpes and I. Andrushchenko, 2013. The Recovery of Atlantic Swordfish: The Comparative Roles of the Regional Fisheries Management Organization and Species Biology. Reviews in Fisheries Science 21(2): 59-97. doi:10.1080/10641262.2012.754842.

Neilson, J. D., S. Smith, F. Royer, S. D. Paul, J. M. Porter and M. Lutcavage, 2009. Investigations of horizontal movements of Atlantic swordfish using pop-up satellite archival tags. In Tagging and tracking of marine animals with electronic devices, pages 145-159. Springer.

Nelson, D. M., J. J. McCarthy, T. M. Joyce and H. W. Ducklow, 1989. Enhanced nearsurface nutrient availability and new production resulting from the frictional decay of a Gulf Stream warm-core ring. Deep Sea Research Part A. Oceanographic Research Papers 36(5): 705-714.

Nielsen, A., K. A. Bigelow, M. K. Musyl and J. R. Sibert, 2006. Improving light-based geolocation by including sea surface temperature. Fisheries Oceanography 15(4): 314325. doi:10.1111/j.1365-2419.2005.00401.x.

Nielsen, A. and J. R. Sibert, 2007. State-space model for light-based tracking of marine animals. Canadian Journal of Fisheries and Aquatic Sciences 64(8): 1055-1068. doi: 10.1139/f07-064.

Nychka, D., R. Furrer, J. Paige and S. Sain, 2015. fields: Tools for spatial data. University Corporation for Atmospheric Research, Boulder, CO, USA. doi:10.5065/D6W957CT. R package version 9.0.

Okubo, A. and S. A. Levin, 2013. Diffusion and ecological problems: modern perspectives, volume 14. Springer Science \& Business Media.

Olson, D., G. Hitchcock, A. Mariano, C. Ashjian, G. Peng, R. Nero and G. Podesta, 1994. Life on the Edge: Marine Life and Fronts. Oceanography 7(2): 52-60. doi:10.5670/ oceanog.1994.03.

Olson, D. B. and R. H. Backus, 1985. The concentrating of organisms at fronts: a cold-water fish and a warm-core Gulf Stream ring. Journal of Marine Research 43(1): 113-137.

Palko, B. J., G. L. Beardsley and W. J. Richards, 1981. Synopsis of the biology of the swordfish, Xiphias gladius Linnaeus. NOAA Technical Report (441).

Palmen, E., 1948. On the formation and structure of tropical hurricanes. Geophysica 3(1): $26-38$.

Parker, H. W. and M. Boeseman, 1954. The basking shark, Cetorhinus maximus, in winter. In Proceedings of the Zoological Society of London, volume 124, pages 185-194. Wiley Online Library. 
Patterson, T. A., M. Basson, M. V. Bravington and J. S. Gunn, 2009. Classifying movement behaviour in relation to environmental conditions using hidden Markov models. Journal of Animal Ecology 78(6): 1113-1123. doi:10.1111/j.1365-2656.2009.01583.x.

Patterson, T. A., B. J. McConnell, M. A. Fedak, M. V. Bravington and M. A. Hindell, 2010. Using GPS data to evaluate the accuracy of state-space methods for correction of Argos satellite telemetry error. Ecology 91(1): 273-285.

Pauly, D., 1998. Fishing Down Marine Food Webs. Science 279(5352): 860-863. doi: 10.1126/science.279.5352.860.

Pauly, D., V. Christensen and S. Guénette, 2002. Towards sustainability in world fisheries. Nature 418(August): 689-695.

Pedersen, M., C. Berg, U. Thygesen, a. Nielsen and H. Madsen, 2011a. Estimation methods for nonlinear state-space models in ecology. Ecological Modelling 222(8): 1394-1400. doi: 10.1016/j.ecolmodel.2011.01.007.

Pedersen, M. W., T. A. Patterson, U. H. Thygesen and H. Madsen, 2011b. Estimating animal behavior and residency from movement data. Oikos 120(9): 1281-1290. doi: 10.1111/j.1600-0706.2011.19044.x.

Pedersen, M. W., D. Righton, U. H. Thygesen, K. H. Andersen and H. Madsen, 2008. Geolocation of North Sea cod (Gadus morhua) using hidden Markov models and behavioural switching. Canadian Journal of Fisheries and Aquatic Sciences 65(11): 2367-2377. doi: 10.1139/f08-144.

Peklova, I., N. E. Hussey, K. J. Hedges, M. A. Treble and A. T. Fisk, 2012. Depth and temperature preferences of the deep-water flatfish Greenland halibut Reinhardtius hippoglossoides in an Arctic marine ecosystem. Marine Ecology Progress Series 467: 193.

Pingree, R. D., P. M. Holligan and G. T. Mardell, 1979. Phytoplankton growth and cyclonic eddies. Nature 278.

Plummer, M., 2004. JAGS: Just another Gibbs sampler User Manual.

Podestá, G. P., J. A. Browder and J. J. Hoey, 1993. Exploring the association between swordfish catch rates and thermal fronts on US longline grounds in the western North Atlantic. Continental Shelf Research 13(2): 253-277.

Polovina, J., I. Uchida, G. Balazs, E. A. Howell, D. Parker and P. Dutton, 2006. The Kuroshio Extension Bifurcation Region: A pelagic hotspot for juvenile loggerhead sea turtles. Deep-Sea Research Part II: Topical Studies in Oceanography 53(3-4): 326-339. doi:10.1016/j.dsr2.2006.01.006.

Polovina, J. J., D. R. Kobayashi, D. M. Parker, M. P. Seki and G. H. Balazs, 2000. Turtles on the edge: movement of loggerhead turtles (Caretta caretta) along oceanic fronts, spanning longline fishing grounds in the central North Pacific, 1997-1998. Fisheries Oceanography 9(1): 71-82.

Priede, I. G., 1984. A basking shark (Cetorhinus maximus) tracked by satellite together with simultaneous remote sensing. Fisheries Research 2(3): 201-216. 
Queiroz, N., N. E. Humphries, G. Mucientes, N. Hammerschlag, F. P. Lima, K. L. Scales, P. I. Miller, L. L. Sousa, R. Seabra and D. W. Sims, 2016. Ocean-wide tracking of pelagic sharks reveals extent of overlap with longline fishing hotspots. Proceedings of the National Academy of Sciences 113(6): 1582-1587. doi:10.1073/pnas.1510090113.

R Development Core Team, 2015. R: A Language and Environment for Statistical Computing.

Reynolds, R. W., N. a. Rayner, T. M. Smith, D. M. Stokes and W. Wang, 2002. An improved in-situ and satellite SST analysis for climate. Journal of Climate 15(July): 1609-1625.

Reynolds, R. W., T. M. Smith, C. Liu, D. B. Chelton, K. S. Casey and M. G. Schlax, 2007. Daily high-resolution-blended analyses for sea surface temperature. Journal of Climate 20(22): 5473-5496. doi:10.1175/2007JCLI1824.1.

Rio, M. H., S. Guinehut and G. Larnicol, 2011. New CNES-CLS09 global mean dynamic topography computed from the combination of GRACE data, altimetry, and in situ measurements. Journal of Geophysical Research: Oceans 116(7): C07018. doi: 10.1029/2010JC006505.

Roman, J. and J. J. McCarthy, 2010. The whale pump: Marine mammals enhance primary productivity in a coastal basin. PLoS ONE 5(10). doi:10.1371/journal.pone.0013255.

Romeo, T., P. Perzia, V. Esposito, D. Malara, P. Battaglia, P. Consoli, S. Canese and F. Andaloro, 2011. Relationship between swordfish swimming behaviour and sea surface temperature in the central Mediterranean Sea during the reproductive period. Marine Biology Research 7(2): 186-194. doi:10.1080/17451000.2010.489615.

Rooker, J. R., J. R. Simms, R. J. D. Wells, S. A. Holt, G. J. Holt, J. E. Graves and N. B. Furey, 2012. Distribution and Habitat Associations of Billfish and Swordfish Larvae across Mesoscale Features in the Gulf of Mexico. PLoS One 7(4): e34180.

Royle, J. A. and R. M. Dorazio, 2008. Hierarchical modeling and inference in ecology: the analysis of data from populations, metapopulations and communities. Academic Press.

Sala, I., C. S. Harrison and R. M. Caldeira, 2016. The role of the Azores Archipelago in capturing and retaining incoming particles. Journal of Marine Systems 154: 146-156. doi:10.1016/j.jmarsys.2015.10.001.

Santos, a. M. P., a. F. G. Fiúza and R. M. Laurs, 2006. Influence of SST on catches of swordfish and tuna in the Portuguese domestic longline fishery. International Journal of Remote Sensing 27(15): 3131-3152. doi:10.1080/01431160600567811.

Scales, K., P. Miller, N. Varo-Cruz, D. Hodgson, L. Hawkes and B. Godley, 2015. Oceanic loggerhead turtles Caretta caretta associate with thermal fronts: evidence from the Canary Current Large Marine Ecosystem. Marine Ecology Progress Series 519: 195-207. doi: $10.3354 /$ meps 11075 .

Scales, K. L., E. L. Hazen, S. M. Maxwell, H. Dewar, S. Kohin, M. G. Jacox, C. A. Edwards, D. K. Briscoe, L. B. Crowder, R. L. Lewison and S. J. Bograd, 2017. Fit to predict? Ecoinformatics for predicting the catchability of a pelagic fish in near real time. Ecological Applications 27(8): 2313-2329. doi:10.1002/eap.1610. 
Scales, K. L., P. I. Miller, C. B. Embling, S. N. Ingram, E. Pirotta and S. C. Votier, 2014. Mesoscale fronts as foraging habitats: composite front mapping reveals oceanographic drivers of habitat use for a pelagic seabird. Journal of the Royal Society, Interface / the Royal Society 11(100): 20140679. doi:10.1098/rsif.2014.0679.

Schirripa, M. J., F. Abascal, I. Andrushchenko, G. Diaz, J. Mejuto, M. Ortiz, M. N. Santos and J. Walter, 2016. A hypothesis of a redistribution of North Atlantic swordfish based on changing ocean conditions. Deep-Sea Research Part II: Topical Studies in Oceanography 140: 139-150. doi:10.1016/j.dsr2.2016.08.002.

Sedberry, G. and J. Loefer, 2001. Satellite telemetry tracking of swordfish, Xiphias gladius, off the eastern United States. Marine Biology 139(2): 355-360.

Seki Michael, P., J. Polovina Jeffrey, R. Kobayashi Donald, R. Bidigare Robert and T. Mitchum Gary, 2002. An oceanographic characterization of swordfish (Xiphias gladius) longline fishing grounds in the springtime subtropical North Pacific. Fisheries Oceanography 11(5): 251-266. doi:10.1046/j.1365-2419.2002.00207.x.

Selkoe, K. A., J. R. Watson, C. White, T. B. Horin, M. Iacchei, S. Mitarai, D. A. Siegel, S. D. Gaines and R. J. Toonen, 2010. Taking the chaos out of genetic patchiness: seascape genetics reveals ecological and oceanographic drivers of genetic patterns in three temperate reef species. Molecular Ecology 19(17): 3708-3726.

Sibert, J. and D. Fournier, 2001. Possible Models for Combining Tracking Data with Conventional Tagging Data, pages 443-456. Springer Netherlands, Dordrecht. doi: 10.1007/978-94-017-1402-0_24.

Sibert, J. R., M. K. Musyl and R. W. Brill, 2003. Horizontal movements of bigeye tuna (Thunnus obesus) near Hawaii determined by Kalman filter analysis of archival tagging data. Fisheries Oceanography 12(3): 141-151.

Sims, D. W., 1999. Threshold foraging behaviour of basking sharks on zooplankton: life on an energetic knife-edge? Proceedings of the Royal Society of London. Series B: Biological Sciences 266(1427): 1437-1443.

Sims, D. W., 2008. Chapter 3 Sieving a Living: A Review of the Biology, Ecology and Conservation Status of the Plankton-Feeding Basking Shark Cetorhinus Maximus. In W. S. David, editor, Advances in Marine Biology, volume Volume 54, pages 171-220. Academic Press. doi:http://dx.doi.org/10.1016/S0065-2881(08)00003-5.

Sims, D. W., E. J. Southall, A. J. Richardson, P. C. Reid and J. D. Metcalfe, 2003. Seasonal movements and behaviour of basking sharks from archival tagging: no evidence of winter hibernation. Marine Ecology Progress Series 248: 187-196.

Sims, D. W., M. J. Witt, A. J. Richardson, E. J. Southall and J. D. Metcalfe, 2006. Encounter success of free-ranging marine predator movements across a dynamic prey landscape. Proceedings of the Royal Society B: Biological Sciences 273(1591): 1195-1201. doi:10. 1098/Rspb.2005.3444.

Skomal, G., C. Braun, J. Chisholm and S. Thorrold, 2017. Movements of the white shark Carcharodon carcharias in the North Atlantic Ocean. Marine Ecology Progress Series 580: 1-16. doi:10.3354/meps12306. 
Skomal, G. B., G. Wood and N. Caloyianis, 2004. Archival tagging of a basking shark, Cetorhinus maximus, in the western North Atlantic. Journal of the Marine Biological Association of the United Kingdom 84(04): 795-799. doi:doi:10.1017/S0025315404009968h.

Skomal, G. B., S. I. Zeeman, J. H. Chisholm, E. L. Summers, H. J. Walsh, K. W. McMahon and S. R. Thorrold, 2009. Transequatorial migrations by basking sharks in the western Atlantic Ocean. Current Biology 19(12): 1019-1022. doi:10.1016/j.cub.2009.04.019.

Smith, A. D. M., C. J. Brown, C. M. Bulman, E. A. Fulton, P. Johnson, I. C. Kaplan, H. Lozano-Montes, S. Mackinson, M. Marzloff, L. J. Shannon, Y.-J. Shin and J. Tam, 2011. Impacts of Fishing Low Trophic Level Species on Marine Ecosystems. Science 333(6046): 1147-1150. doi:10.1126/science.1209395.

Smith, B. L., C. P. Lu, B. García-Cortés, J. Viñas, S. Y. Yeh and J. R. Bremer, 2015. Multilocus bayesian estimates of intra-oceanic genetic differentiation, connectivity, and admixture in Atlantic Swordfish (Xiphias gladius L.). PLoS ONE 10(6): 1-30. doi: 10.1371/journal.pone.0127979.

Smith, P. and D. Goodman, 1986. Determining fish movements from an" archival" tag: precision of geographical positions made from a time series of swimming temperature and depth. US Department of Commerce, National Oceanic and Atmospheric Administration, National Marine Fisheries Service, Southwest Fisheries Center.

Squire Jr, J. L., 1990. Distribution and apparent abundance of the basking shark, Cetorhinus maximus, off the central and southern California coast, 1962-85. Marine Fisheries Review 52(2): 8-11.

Stillwell, C. E. and N. E. Kohler, 1985. Food and feeding ecology of the swordfish Xiphias gladius in the western North Atlantic Ocean with estimates of daily ration. Marine Ecology Progress Series 22(3): 239-247.

Stommel, H., 1963. Varieties of oceanographic experience. Science 139(3555): 572-576.

Stramma, L., E. D. Prince, S. Schmidtko, J. Luo, J. P. Hoolihan, M. Visbeck, D. W. R. Wallace, P. Brandt and A. Kortzinger, 2012. Expansion of oxygen minimum zones may reduce available habitat for tropical pelagic fishes. Nature Climate Change 2(1): 33-37.

Suca, J., L. Rasmuson, E. Malca, T. Gerard and J. Lamkin, 2017. Characterizing larval swordfish habitat in the western tropical North Atlantic. Fisheries Oceanography .

Sumner, M. and S. Luque, 2015. Package "Trip: Spatial analysis of animal track data", r package version 1.5 edition.

Sumner, M. D., S. J. Wotherspoon and M. A. Hindell, 2009. Bayesian estimation of animal movement from archival and satellite tags. PLoS One 4(10): e7324. doi:10.1371/journal. pone.0007324.

Talley, L. D., 2011. Descriptive physical oceanography: an introduction. Academic press.

Templeman, W., 1963. Distribution of Sharks in the Canadian Atlantic: With Special Reference to Newfoundland Waters. Fisheries Research Board of Canada. 
Teo, S., a. Boustany, S. Blackwell, a. Walli, K. Weng and B. Block, 2004. Validation of geolocation estimates based on light level and sea surface temperature from electronic tags. Marine Ecology Progress Series 283: 81-98. doi:10.3354/meps283081.

Teo, S. L. H. and B. A. Block, 2010. Comparative influence of ocean conditions on yellowfin and Atlantic bluefin tuna catch from longlines in the Gulf of Mexico. PLoS One 5(5): e10756.

Teo, S. L. H., A. Boustany, H. Dewar, M. J. W. Stokesbury, K. C. Weng, S. Beemer, A. C. Seitz, C. J. Farwell, E. D. Prince and B. A. Block, 2007. Annual migrations, diving behavior, and thermal biology of Atlantic bluefin tuna, Thunnus thynnus, on their Gulf of Mexico breeding grounds. Marine Biology 151(1): 1-18.

Tew Kai, E., V. Rossi, J. Sudre, H. Weimerskirch, C. Lopez, E. Hernandez-Garcia, F. Marsac and V. Garcon, 2009. Top marine predators track Lagrangian coherent structures. Proceedings of the National Academy of Sciences 106(20): 8245-8250. doi: 10.1073/pnas.0811034106.

Thorne, L. H. and A. J. Read, 2013. Fine-scale biophysical interactions drive prey availability at a migratory stopover site for Phalaropus spp. in the Bay of Fundy, Canada. Marine Ecology Progress Series 487: 261-273.

Thorrold, S. R., P. Afonso, J. Fontes, C. D. Braun, G. B. Skomal and M. L. Berumen, 2014. Extreme diving behavior in devil rays link surface waters and the deep ocean. Nature Communications 5(4274). doi:10.1038/ncomms5274.

Thygesen, U. H., M. W. Pedersen and H. Madsen, 2009. Geolocating Fish Using Hidden Markov Models and Data Storage Tags, pages 277-293. Springer Netherlands, Dordrecht. doi:10.1007/978-1-4020-9640-2_17.

Tyack, P. L., M. Johnson, N. A. Soto, A. Sturlese and P. T. Madsen, 2006. Extreme diving of beaked whales. Journal of Experimental Biology 209(21): 4238-4253.

Viterbi, A. J., 2006. A personal history of the Viterbi algorithm. IEEE Signal Processing Magazine 23(4): 120-142.

Watson, R. A., W. W. L. Cheung, J. A. Anticamara, R. U. Sumaila, D. Zeller and D. Pauly, 2013. Global marine yield halved as fishing intensity redoubles. Fish and Fisheries 14(4): 493-503. doi:10.1111/j.1467-2979.2012.00483.x.

WC, 2015. Data Portal's Location Processing (GPE3 \& FastLoc-GPS) User Guide. Technical report, Wildlife Computers, Inc.

Werry, J. M., S. Planes, M. L. Berumen, K. A. Lee, C. D. Braun and E. Clua, 2014. ReefFidelity and Migration of Tiger Sharks, Galeocerdo cuvier, across the Coral Sea. PLoS One 9(1): e83249. doi:10.1371/journal.pone.0083249.

Wilk, S. J., A. L. Pacheco and B. Baker, 1988. Fish and fisheries of the Middle Atlantic Bight. Technical report, Characterization of the Middle Atlantic Water Management Unit of the Northeast Regional Action Plan. NOAA Tech. Mem. NMFS-F/NEC-56.

Williams, R. G. and M. J. Follows, 1998. Eddies make ocean deserts bloom. Nature 394: 228. 
Wilson, S. G., 2004. Basking sharks (Cetorhinus maximus) schooling in the southern Gulf of Maine. Fisheries Oceanography 13(4): 283-286.

Wilson, S. G., B. S. Stewart, J. J. Polovina, M. G. Meekan, J. D. Stevens and B. Galuardi, 2007. Accuracy and precision of archival tag data: a multiple-tagging study conducted on a whale shark (Rhincodon typus) in the Indian Ocean. Fisheries Oceanography 16(6): 547-554. doi:10.1111/j.1365-2419.2007.00450.x.

Winship, A. J., S. J. Jorgensen, S. A. Shaffer, I. D. Jonsen, P. W. Robinson, D. P. Costa and B. A. Block, 2012. State-space framework for estimating measurement error from double-tagging telemetry experiments. Methods in Ecology and Evolution 3(2): 291-302. doi:10.1111/j.2041-210X.2011.00161.x.

Witt, M. J., S. Åkesson, A. C. Broderick, M. S. Coyne, J. Ellick, A. Formia, G. C. Hays, P. Luschi, S. Stroud and B. J. Godley, 2010. Assessing accuracy and utility of satellitetracking data using Argos-linked Fastloc-GPS. Animal behaviour 80(3): 571.

Witt, M. J., T. Hardy, L. Johnson, C. M. McClellan, S. K. Pikesley, S. Ranger, P. B. Richardson, J.-L. Solandt, C. Speedie and R. Williams, 2012. Basking sharks in the northeast Atlantic: spatio-temporal trends from sightings in UK waters. Marine Ecology Progress Series 459: 121-134.

Woillez, M., R. Fablet, T. T. Ngo, M. Lalire, P. Lazure and H. de Pontual, 2016. A HMMbased model to geolocate pelagic fish from high-resolution individual temperature and depth histories: European sea bass as a case study. Ecological Modelling 321: 10-22. doi:10.1016/j.ecolmodel.2015.10.024.

Wood, S., 2006. Generalized additive models: an introduction with R. CRC press.

Worm, B., 2005. Global Patterns of Predator Diversity in the Open Oceans. Science 309(5739): 1365-1369. doi:10.1126/science.1113399.

Wysujack, K., H. Westerberg, K. Aarestrup, J. Trautner, T. Kurwie, F. Nagel and R. Hanel, 2015. The migration behaviour of European silver eels (Anguilla anguilla) released in open ocean conditions. Marine and Freshwater Research 66(2): 145-157. doi:10.1071/MF14023.

Zucchini, W. and I. L. MacDonald, 2009. Hidden Markov models for time series: an introduction using $R$, volume 22. CRC Press, Boca Raton.

Zuur, A. F., E. N. Ieno, N. J. Walker, A. A. Saveliev and G. M. Smith, 2009. Mixed effects models and extensions in ecology with R. Gail M, Krickeberg K, Samet JM, Tsiatis A, Wong W, editors. New York, NY: Spring Science and Business Media . 